\title{
Nanocapillary Membrane Devices: A Study in Electrokinetic Transport Phenomena
}

Jarrod Schiffbauer

West Virginia University

Follow this and additional works at: https://researchrepository.wvu.edu/etd

\section{Recommended Citation}

Schiffbauer, Jarrod, "Nanocapillary Membrane Devices: A Study in Electrokinetic Transport Phenomena" (2011). Graduate Theses, Dissertations, and Problem Reports. 3472.

https://researchrepository.wvu.edu/etd/3472

This Dissertation is protected by copyright and/or related rights. It has been brought to you by the The Research Repository @ WVU with permission from the rights-holder(s). You are free to use this Dissertation in any way that is permitted by the copyright and related rights legislation that applies to your use. For other uses you must obtain permission from the rights-holder(s) directly, unless additional rights are indicated by a Creative Commons license in the record and/ or on the work itself. This Dissertation has been accepted for inclusion in WVU Graduate Theses, Dissertations, and Problem Reports collection by an authorized administrator of The Research Repository @ WVU.

For more information, please contact researchrepository@mail.wvu.edu. 


\title{
Nanocapillary Membrane Devices: A Study in Electrokinetic Transport Phenomena
}

\author{
by \\ Jarrod Schiffbauer \\ Dissertation submitted to the \\ College of Arts and Sciences \\ at West Virginia University \\ in partial fulfillment of the requirements \\ for the degree of \\ Doctor of Philosophy \\ in \\ Physics \\ Paul Cassak, Ph.D. \\ Mark Koepke, Ph.D. \\ Aaron Timperman, Ph.D. \\ Arthur Weldon, Ph.D. \\ Boyd Edwards, Ph.D., Chair \\ Physics Department \\ Morgantown, West Virginia \\ 2011
}

Keywords: Electrokinetics, microfluidics, fluidic rectifier

Copyright 2011 Jarrod Schiffbauer 


\author{
Abstract \\ Nanocapillary Membrane Devices: \\ A Study in Electrokinetic Transport Phenomena \\ by \\ Jarrod Schiffbauer
}

There is considerable interest in developing micro-total analysis systems, also known as lab-on-a-chip devices, for applications in chemical and biological analysis. These devices often employ electrokinetic transport phenomena to move, mix, concentrate and separate dissolved species. The details of these phenomena in micro- and nanometer scale geometries are not fully understood; consequently, the basic principles of device operation are often unclear. For example, nanocapillary membranes (NCM) and other nanometer-sized passages can exhibit charge-selectivity and rectification effects similar to those observed in biological membranes. This dissertation addresses several issues related to ion transport in these membranes. Leading-order 1D steady-state models for diffusion-layer modulated transport through non-ideal membranes are used to study ionic rectification in geometrically asymmetric devices. These models provide qualitative explanations of the operation of a variety of fluidic rectifiers and experimentally observed hysteresis effects. By taking the first steps in the full boundary-layer analysis of the model, it is shown that non-ideal membranes do not maintain local electro-neutrality under passage of electric current. This is in contrast to the usual assumption of membrane local electro-neutrality, but is compatible with the existence of the non-equilibrium macroscopic space charge known to appear in the flanking electrolyte and the requirement of overall charge conservation. Lastly, the problem of electrokinetic instability due to non-equilibrium electro-osmotic slip is considered for the case of an electrolyte-membrane interface inside a 2D channel. 


\section{Acknowledgements}

I would like to thank my advisor and committee chair, Dr. Boyd Edwards, for providing me with the opportunity to work on these problems, for support and encouragement along the way, and for providing an all-around excellent role model for an aspiring academic. I would also like to thank my committee members, Dr. Paul Cassak, Dr. Mark Koepke, and Dr. Arthur Weldon for their time, advice and comments. I would like to thank Dr. Aaron Timperman for sitting on my committee, but more importantly, for providing me with interesting experiments to study, access to his lab, and his and his students' time. Next I would like to thank Dr. Isaak Rubinstein and Dr. Boris Zaltzman who hosted me at Ben Gurion University for a critical month. A large portion of this work would not have been possible without their guidance; I learned a tremendous amount working with them and greatly enjoyed their hospitality. I would also like to thank the other faculty members, post-docs, and students in our promising yet seemingly short-lived micro-fluidics research group here at WVU. It's been a pleasure working with them over the years. In particular, I would like to thank my colleagues Dr. Kathleen Reschke and Will Booth, both of whom have been very helpful throughout this project. Finally, I would like to express my gratitude to my family and friends. They are the most important people in my life and have always been there to provide encouragement and support. My wife and parents deserve special thanks for their patience with me over the years, both before and throughout my doctoral studies, and words are insufficient to express my gratitude to them. 


\section{Contents}

$\begin{array}{ll}\text { Acknowledgements } & \text { iii }\end{array}$

List of Figures vii

List of Tables $\quad$ xiii

1 Introduction $\quad 1$

1.1 Introduction . . . . . . . . . . . . . . . . . . . 1

1.1.1 Applications: LOC/ $\mu$-TAS technology . . . . . . . . . . . 1

1.1.2 Electrokinetic transport in micro- and nanometer-sized geometries . . 2

1.2 The NCM/NMI device . . . . . . . . . . . . . . . . . 5

1.2.1 Device construction, experimental details, etc. . . . . . . . . . 5

1.2.2 On- and Off-state Illustrations . . . . . . . . . . . . . . . . . 5

$1.3 \mathrm{NCM} / \mathrm{NMI}$ applications and experiments . . . . . . . . . . . . . 7

1.4 Overview of dissertation . . . . . . . . . . . . . . . . . . 8

2 Background theory and related problems $\quad 10$

2.1 Chapter introduction and overview . . . . . . . . . . . . . . 10

2.2 Continuum models of electrokinetics . . . . . . . . . . . . . . 10

2.2.1 The governing equations . . . . . . . . . . . . . . . . . 11

2.2.2 Dimensionless forms and important parameters . . . . . . . . . . . 12

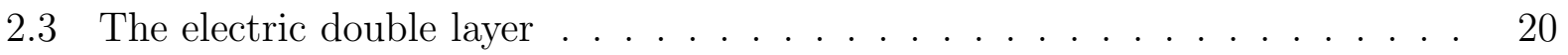

2.3.1 Thermodynamic basis for boundary layers . . . . . . . . . . . . . 20

2.3.2 Equilibrium electric double-layers: Poisson-Boltzmann equation and the Gouy-Chapman theory of the electric double-layer . . . . . . . . . 23

2.3.3 Permselectivity near equilibrium and simple models for the NCM . . 28

2.4 Non-equilibrium electro-diffusion through charge-selective membranes . . . . 30

2.4.1 Concentration polarization and (quasi-) steady-state membrane-electrolyte $I-V$ characteristics . . . . . . . . . . . . . . . . . . . . . . . . . . 31

2.4.2 The problem with locally electro-neutral membranes and the existence of extended space charge . . . . . . . . . . . . . . . . . . . 37

2.4.3 Transient effects, diffusion-layer dynamics, and a quasi-static approx-

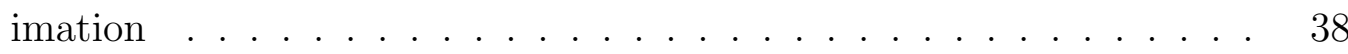


3 A classical electro-diffusive model of fluidic rectification 43

3.1 Chapter introduction and overview . . . . . . . . . . . . . 43

3.2 A review of fluidic rectification . . . . . . . . . . . . . . . . 44

3.2.1 Historical development, device types . . . . . . . . . . . . . 44

3.2.2 Comparison to the NCM/NMI: Flux- and field-focusing geometries . 49

3.2.3 Comparison of theoretical explanations, models, and experiments . . 50

3.3 The relationship between geometric asymmetry and asymmetric concentration polarization in the $\mathrm{NCM} / \mathrm{NMI}$ device . . . . . . . . . . . . 55

3.3.1 Flux conservation in a geometrically asymmetric discontinuous system 57

3.3.2 Using Hunter's result to compare the DL lengths . . . . . . . . . . 58

3.4 Rectification due to asymmetric concentration polarization using a 1D model 60

3.4 .1 The 1D LEN model equations . . . . . . . . . . . . . . . . . 61

3.4 .2 Geometry and boundary conditions . . . . . . . . . . . . . 62

3.4 .3 The $\mathrm{CP}$ solution in the diffusion layers . . . . . . . . . . . . . 63

3.4 .4 The ideal membrane case . . . . . . . . . . . . . . . . . 66

3.4.5 The non-ideal membrane case . . . . . . . . . . . . . . . . . . . . 69

3.4.6 Limit of large $\mathrm{N}$ for non-ideal membrane . . . . . . . . . . . . . 75

3.4 .7 Ohmic behavior in the low-voltage limit . . . . . . . . . . . . 79

3.5 Including flux-focusing effects . . . . . . . . . . . . . . . . 83

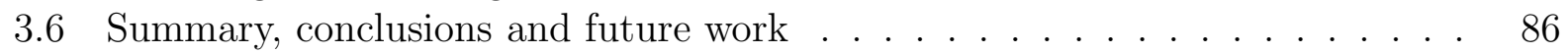

3.6 .1 Summary and conclusions . . . . . . . . . . . . . 86

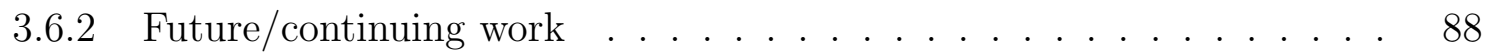

4 Analysis of experimental data $\quad 92$

4.1 Chapter introduction and overview . . . . . . . . . . . . . . . . 92

4.2 Overview of experimental details . . . . . . . . . . . . . . . 93

4.2.1 A brief description of the experimental set-up and process . . . . . 93

4.2 .2 A typical I-t curve for the NCM/NMI . . . . . . . . . . . . . 96

4.3 Fluidic rectification in two types of asymmetric PC-NCM and Nafion membrane device . . . . . . . . . . . . . . . . . . . . 100

4.3.1 Top-mounted PC-NCM device . . . . . . . . . . . . . . . . . . . 100

4.3 .2 Side-mounted Nafion device . . . . . . . . . . . . . . . . . . . . 105

4.3.3 Comparison of the current density and low-voltage rectification . . 108

4.4 Rectification and hysteresis in an asymmetric side-mounted PC-NCM device 112

4.4 .1 Rectification . . . . . . . . . . . . . . . . . . . . . . 112

4.4 .2 Varying bulk concentration . . . . . . . . . . . . . . . . . 116

4.4.3 Chemical rachet: electrokinetic hysteresis . . . . . . . . . . . . . . . 119

4.5 High-voltage off-state analyte concentration . . . . . . . . . . . . . 122

4.5.1 Some notes on use of fluorescent imaging to obtain information about concentrations . . . . . . . . . . . . . . . . . . . 123

4.5.2 Analyte concentration process at -800V . . . . . . . . . . . 124

4.6 Summary, conclusions, future work . . . . . . . . . . . . . . . 132

4.6 .1 Summary and conclusions . . . . . . . . . . . . . . . . . . 132

4.6 .2 Future/continuing work . . . . . . . . . . . . . . . . 134 
5 Beyond the classical approximation $\quad 137$

5.1 Chapter introduction and overview . . . . . . . . . . . . . . . 137

5.2 The effects of space charge at increasing fluxes and breakdown of the Q1DL description . . . . . . . . . . . . . . . . . 138

5.3 Finite- $\epsilon$ formulation of problem for non-ideal membranes . . . . . . . . . . . 142 5.3.1 The electrolyte layers outside the membrane . . . . . . . . . . . 145

5.3.2 The membrane interior . . . . . . . . . . . . . . . . . 150

5.4 Electro-osmotic instability of a confined electrolyte due to non-equilibrium electro-osmotic slip . . . . . . . . . . . . . . . . . 154

5.5 Discussion, conclusions, and future work . . . . . . . . . . . . . 162

A Mathematica codes

A.1 Numerical evaluation for the 1D and quasi-1D LEN non-ideal solution . . . . 164

A.2 Version of the code for plotting concentration and potential profiles . . . . . 169 


\section{List of Figures}

1.1 Schematic On- and Off- state device illustrations showing the basic relationship between species flux densities $j_{ \pm}$, i.e. number of particles per unit area per unit time, and salt flux density, $J_{+} \equiv\left(j_{+}+j_{-}\right) / 2$, and charge flux density, $J_{-} \equiv\left(j_{+}-j_{-}\right) / 2$ for a two-species system. The depleted and enriched regions are indicated respectively by lighter and darker shades of blue. . . . . . . . .

1.2 Fluorescent microscope image of dye concentrated in the on-state in a Vchannel device with a single nanochannel as the perm-selective element. The device operation is essentially the same as that in the NCM/NMI on-state. Image courtesy of K. Kelly (unpublished.) . . . . . . . . . . . . . . . .

2.1 Thermo-mechanical double-layers (green box) formed by chemical and physical relaxation at the boundary between basaltic lava and the surrounding limestone, resulting in a macroscopic region - the darker flint-like materialof different appearance and composition than either of the two 'bulk' materials. Similar features can be found in many of the rock formations of the Negev desert. . . . . . . . . . . . . . . . . . . . . . . . . .

2.2 Schematic EDL anatomy showing various important features in the electric double-layer for a fixed negative surface charge, including the variation in potential from the so-called surface of shear which separates the compact, relatively immobile Stern layer from the diffuse portion of the double-layer. The zeta potential is defined as the value of the electric potential at this surface. This picture is fundamentally similar to the usual one appearing first in Ref. [17] . . . . . . . . . . . . . . . . . . . . . . 
2.3 Anatomy of 1D concentration polarization layers (CPL) near a cation-selective membrane showing the LEN salt concentration in black and the cation (red) and anion (blue) concentrations. Five distinct regions are identified. Regions 1 denote electrolyte at bulk concentrations. This situation is somewhat artificial in the sense that $\mathrm{CP}$ will spread out from the electrodes towards the membrane as well, however if the electrodes are in large enough reservoirs located far from the membrane, the picture is realistic enough. Following the terminology of Ref. [68], regions 2 and 3 are the left and right quasi-1D diffusion layers (Q1DL) and regions 4 are known as the quasi-electroneutral bulk (QEB), which may be taken to be the same as the diffusion layers when spacecharge can be ignored. Regions 5 correspond to the charged Debye layers and the depleted membrane-electrolyte interface can thicken above the classical limiting current. . . . . . . . . . . . . . . . . . .

2.4 Typical form for a classical $I$ - $V$ curve showing the diffusion-limited current saturation associated with the decrease of interfacial ion concentration. . . .

2.5 Typical features seen in an experimental $I-V$ curve for a membrane-electrolyte system with a sufficiently non-viscous fluid (i.e. water) as the solvent. (a) At low voltages, there is an Ohmic response region. (b) The limiting current begins to saturate near a critical voltage. Depending on the system, the limiting current region may have either a small or pronounced slope, but typically less than the slope in the Ohmic region. (c) Finally, at a second critical voltage, charge-selective systems typically develop a severe over-limiting response with a slope equal to or higher than the Ohmic slope. Note the over-limiting region may be suppressed by using a highly viscous fluid or gel instead of a relatively in-viscid aqueous electrolyte.[53] . . . . . . . . . . . . . . .

3.1 Flux-focusing geometries for several theoretical and experimental studies of fluidic rectification. (a) Wei's infinite-cone model, (b) A single channel 'cell' for the nanochannel array of Yossifon and Chang, (c) the NCM/NMI, (d) Jung et. al's thimble, and (e) Siwy et. al's conical pores. . . . . . . . . . . .

3.2 On and off cartoons using Wei's geometry and Pu's intuitive flux balancing

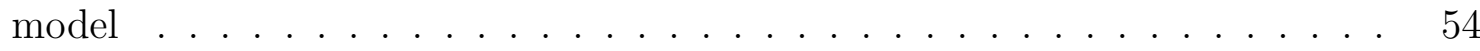

3.3 An asymmetric discontinuous geometry similar to that in the NCM/NMI. . .

3.4 A plot of the diffusion-layer length asymmetry versus ratio of cross-sectional areas according to the quasi-1D argument. Data points show values from a preliminary 2D FEM calculation . . . . . . . . . . . . . . . . . . . . . . . . . . . .

3.5 Geometry of the model system showing each region . . . . . . . . . . . . . .

3.6 Ideal membrane I-V curve showing rectification dependent on DL length asymmetry. $I=-j$ is the dimensionless current density (zero co-ion flux.) The parameters are $N=10, \mathcal{D}=0.1$, and $\ell=1.5 \ldots \ldots \ldots$

3.7 Non-ideal membrane IV curve for systems with several values of asymmetry $\gamma$, and fixed charge density $N=10$, effective diffusivity $\mathcal{D}=0.2$, and $\ell=$ 1.5. The variation of selectivity with voltage, along with a legend for the asymmetry values, is shown in the inset. . . . . . . . . . . . . . 
3.8 Concentration (top) and electric potential (bottom) profiles for the off- and on- states near the limiting current for the same parameter values as the previous plot, with $\gamma=6.33$. The cation concentration is shown in red, the anion concentration in blue and the reduced (salt) concentration in black. The membrane edges are denoted by vertical lines at $x= \pm 1$.Note the strong concentration polarization in the on-state $\mathrm{CP}$ profile and the ratchet- like potential in the off-state. . . . . . . . . . . . . . . . .

3.9 Non-ideal cases (solid lines) for several values of membrane charge density $N$ $\gamma=6.3333$, and $\ell=1.5$ and adjusting the membrane diffusivity to keep the product $\mathcal{D} N=1$. For comparison, the red plot shows the ideal membrane case for the same conductivity . . . . . . . . . . . . . . . . . .

3.10 IV characteristics for non-ideal membrane with flux-focusing effect only, assuming same DL lengths, $\gamma=1$ and varying the area ratio $\Gamma$ only. All other

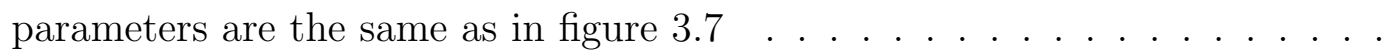

$3.11 \mathrm{I}-\mathrm{V}$ characteristics and rectification factor for several $\mathrm{N}$ comparing flux-focusing to DL length asymmetry alone . . . . . . . . . . . . . . . .

4.1 Diagram showing geometries and approximate dimensions for the two device configurations used in the experiments, (a) the side-mounted membrane device and (b) the top-mounted membrane device . . . . . . . . . . . . .

4.2 Schematic of experimental set-up for data collection. Note: NMI fluidic rectifier represented by a the symbol for a Schottky diode with the ' + ' denoting cation-selectivity. . . . . . . . . . . . . . . . . .

4.3 I-t response for a 40 micron channel with $10 \mathrm{mM}$ buffer with stepped voltage applied showing several typical features for the NCM/NMI. Note the voltage intervals are not all equal; $10 \mathrm{~V}$ steps are used in the off-state and $2 \mathrm{~V}$ steps in the on-state. . . . . . . . . . . . . . . . . . . . . . .

4.4 I-t curve for a top-mounted PC-NCM/NMI showing several distinct response regimes. In particular, note the differences between the regions $-400 \mathrm{~V}$ to $-100 \mathrm{~V}$ and between 100V-and 400V. . . . . . . . . . . . . . .

4.5 Interpolated I-V curve for top-mounted NCM device (solid line) with averaged $\mathrm{I}-\mathrm{V}$ data points. Dashed lines are linear extrapolations of the $\mathrm{I}-\mathrm{V}$ behavior on either side of the origin, the slopes of which are almost indistinguishable by inspection. . . . . . . . . . . . . . . . . .

4.6 Rectification factor for top-mounted NCM device, interpolated (solid line), data points (points,) and extrapolated experimental $V=0$ limit (dashed line.) 104

4.7 I-t curve for a side-mounted Nafion device. Note the relatively steady currents (albeit with barely perceptible slope at voltages as low as 100V) up to voltages close to $300 \mathrm{~V}$ and the marked increase between $600 \mathrm{~V}$ and $700 \mathrm{~V}$. The off-state transients appear to be suppressed. . . . . . . . . . . . . . . . (solid line), time-averaged I-V data points, and extrapolated $V=0$ lines.

4.8 Time-averaged and interpolated I-V curve for a side-mounted Nafion device 
4.9 Rectification factor for side-mounted Nafion device, interpolation (solid line,) data points (points,) and the classical (theoretical) low-voltage limit, $R=1$, (dashed.) Note the Nafion device approaches this limit much more convincingly than the NCM, which may indicate the dominance of electro-diffusive transport in the former and the importance of EOF in the latter . . . . . .

4.10 Comparison of current density vs. voltage, w/ current normalized by channel cross-section. Inset shows very low-voltage behavior, though given the sparsity of data points, that the Nafion current density appears to be lower than the NCM current density at very low voltages must be taken with a grain of salt.

4.11 Rectification factor for both devices at low voltage with the low-voltage LEN limit indicated by the dashed line. Note that the Nafion case bears a close resemblance to the LEN models below about 50-60V and approaches the classical limit much more convincingly than the NCM. This may be some indication of space-charge/electro-convective effects appearing well below the high voltage range, however still considerably higher than the typical classical limit for an ideal membrane. . . . . . . . . . . . . . . . . . . . .

4.12 On-state current density vs. time for stepped voltages for the three microchannel sizes, $10 \mathrm{mM}$ buffer. $2 \mathrm{~V}$ steps shown. Note the apparent multiple I-t inflections in the 12 micron case. . . . . . . . . . . . . . . .

4.13 Averaged current density vs. voltage for the three microchannel sizes, $10 \mathrm{mM}$ buffer. The 12 micron channel off-state for the side-mounted NCM has an unusually high current density. . . . . . . . . . . . . . . . . .

4.14 Rectification factors for the side-mounted NCM device with three different microchannel depths, $10 \mathrm{mM}$ buffer. The unusually high off-state current densities (by comparison with the top-mounted NCM and side-mounted Nafion membrane) seem to be the source of difficulty. The on-state densities, below the inflections, are comparable to those in the top-mounted device at higher voltages. . . . . . . . . . . . . . . . . . . . . . .

4.15 Averaged I-V curves for the 40 micron side-mounted NCM device at three different concentrations. The I-t inflection point was not visible on the $1 \mathrm{mM}$ case, but occurred between $18 \mathrm{~V}$ and $20 \mathrm{~V}$ for the $10 \mathrm{mM}$ and $20 \mathrm{mM}$ cases. . .

4.16 Rectification factors for the 40 micron side-mounted NCM at three different concentrations. The behavior near $V=0$ is an artifact of the interpolation. The dashed line shows the theoretical low-V limit of chapter $2 . . . .118$

4.17 Experimental observation of chemical ratchet effect in a 40 micron side-mounted device at $1 \mathrm{mM}$ buffer concentration. The runs were performed in (somewhat) rapid succession, starting at the largest negative voltage, where the system sat for 10 minutes (first five minutes not shown) before stepping up through the sequence of voltage. . . . . . . . . . . . . . . .

4.18 Calculated chemical ratchet effect using arbitrary parameters and the ideal model to demonstrate the basic mechanism . . . . . . . . . . . . . . . 
4.19 Post-processed contour intensity maps of fluorescent microscope images at several time frames during the initial transient phase of analyte concentration in the high-voltage off-state. The membrane is located at the far right side of the figure and the distance from the membrane back through the channel given in microns. Undulations, characteristic of vortex-like instabilities in the depleted region, are evident at the side of the plug closest to the membrane. Note also these images do not show the full channel width, this reduced box was used to improve the resolution of the image. Lastly, in the last frame, an apparent ghost image due to image saturation appears trailing the actual plug by about 400 microns. . . . . . . . . . . . . . . . . . . .

4.20 Plot of the transient current pulse during this time period. By about 15 seconds, the system achieves what appears to be a quasi-steady value. The initial transient corresponds to the formation of the depleted region. . . . . .

4.21 Plot of the position of the plug mean vs. time obtained from a 1D average of the data presented in Figure 4.19. The change in the position of the mean may be used as a very rough estimate of changes in the spatial extent of the depleted region. Note that several distinct regimes of behavior are apparent in this figure. After about 17 seconds, the plug position remains roughly constant for the duration of the experiment. . . . . . . . . . . . . . . .

4.22 Plot showing the variance, proportional to the full-width half-maximum, of the plug. These were extracted from the 1D data by calculating the moments of the 1D averaged distribution. The rapid contraction in both this plot and the previous one are indicative of the rapid build-up of an extended space charge adjacent to the membrane. . . . . . . . . . . . . . . . . . . . . . 129

4.23 A simple experimental device to test the 1D steady-state theory. . . . . . . . 135

4.24 A device similar to the Yossifon et. al. design for investigating the relationship between geometry, time-dependent DL length asymmetry, and fluidic rectification. Note that the time intervals over which the experiments should be conducted are dependent upon device geometry. This is to ensure that the slow (sub-critical, electro-neutral) diffusion layers do not reach the electrodes so that the idea of a propagating diffuse front may be investigated. Presumably, these restrictions require modification for tests above the LEN threshold voltages, as per the theory in Ref. [49] . . . . . . . . . . . . . 136 
5.1 1D geometry showing matching, boundary, and jump conditions for the singularly perturbed electro-diffusive problem at an interface with a non-ideal membrane. The regions shown are: (a) and (c), the left and right electrolyte layers respectively, i.e. assumed to be quasi-electroneutral bulk (QEB) (b) the membrane interior, $(\mathrm{d})$ the inner region up to the extended space charge outside the membrane, and (e) the inner region inside the membrane. (f) is the $Q 1 D L \cap Q E B$ overlap region. Note that the point $x_{o}$ is shown to correspond to the outer edge of the extended space charge here, and that this picture is valid then for currents above the classical limiting value. The point $x_{i}$ is assumed to be at the right membrane-solution boundary, however, this picture may need revision following a rigorous and complete boundary layer

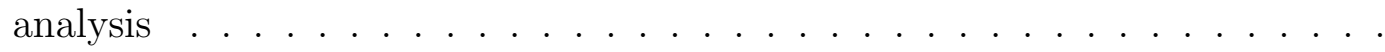

5.2 2D channel geometry and boundary conditions for the stability problem. Notice the membrane/non-equilibrium double-layer are located at the origin, left-side of the figure, with the positive y-direction along the channel. No slip and no flux boundary conditions are imposed on the walls and axial symmetry is assumed. . . . . . . . . . . . . . . . . . . . . 


\section{List of Tables}

3.1 Estimates of characteristic time for diffusion of various ions present in the NCM/NMI experiments for $1 \mathrm{~cm}$ and 100 micron lengths. According to the universal scaling limit from Hunter's analysis [?], which is expected to be approximately valid for systems operating below the classical limiting current, this gives an estimate of the time required for diffusion layer growth across such distances. . . . . . . . . . . . . . . . . . . . . . . . . .

5.1 Important threshold voltage estimates for concentrations $c_{o} \sim 1 \mathrm{mM}$ and $10 \mathrm{mM}$ giving Debye lengths of approximately $3.36 \mathrm{~nm}$ and $10 \mathrm{~nm}$ respectively. The numbers shown for the thresholds are voltages, i.e. the dimensionless orders $\times 0.0254 \mathrm{~V}$, i.e. the thermal voltage. . . . . . . . . . . . .

5.2 Governing effective diffusion length estimates for the asymmetric side-mounted PC-NCM devices assuming the maximum estimated voltage for the Q1DL corresponds to the I-t inflection. . . . . . . . . . . . . . . . . . . . 140

A.1 Model parameters from Chapter 2 and Mathematica variable names. . . . . 165 


\section{Chapter 1}

\section{Introduction}

\section{$1.1 \quad$ Introduction}

\subsubsection{Applications: LOC/ $\mu$-TAS technology}

Microfluidic technology emerged about 20 years ago with the development of micronsized chromatography systems, micropumps, valves, and flow sensors [39] and has since transitioned into commonplace technology; for instance, the ink-jet printer employs an array of micron-diameter channels. A more recent technological trend, driven by industrial needs, is to integrate small-scale versions of separation, mixing, concentration, and analysis processes into, ideally, a microchip-sized device. Such technology, known colloquially as Lab-On-a-Chip (LOC) or Micro-Total Analysis Systems ( $\mu$-TAS) could potentially increase efficiency and decrease cost in a wide array of applications such as those involving remote chemical or biological sensing and detection, DNA sequencing, or portable/disposable medical diagnostics.

The archetypal LOC device consists of several individual components performing the separation, mixing, concentration, pumping, and analysis processes integrated into a single platform and connected by channels with cross-sectional areas on the order of $1000 \mu \mathrm{m}^{2}$ and lengths around $1 \mathrm{~cm}$ or less. At these scales, it is desirable to avoid mechanical pumps and valves both because of the inherent difficulties in the precision fabrication and reliable 
function of complex devices, but also for reasons having to do with optimization in separations science, which will be discussed subsequently. Instead, advantage is taken of the novel transport properties which can dominate in such systems. The components themselves may be formed of such micro-channels and chambers with particular shapes, surface modifications, or as in the case of current interest, nanofluidic interconnects to facilitate the various intended functions.

Fortunately, the challenges imposed by miniaturization of these processes entails the study of a number of interesting problems. Strictly speaking, even though the smaller dimensions in these systems may reach nanometer scales, most of these are not so much nanoscale effects in the sense that atomistic effects or fluctuations become important. They are however the result of interfacial effects becoming increasingly important as the device size decreases; the surface area to volume ratio increases and boundary-layer transport becomes important.

For many practical applications, particularly related to biological sample concentration and separation, samples are dissolved in aqueous electrolytic solutions. The parabolic flow profile of a pressure-driven flow tends to disperse samples faster, via Taylor dispersion [58], than the uniform flow profiles generated by the collective forces exerted by the charged salt ions on the solution, i.e. electro-osmotic flow (EOF). Thus EOF and electrophoresis are often used to move and control samples in LOC systems. Because of this, micro- and nanofluidic devices, particularly devices involving the nano-microfluidic interface (NMI) provide ample opportunity to study electrokinetic transport in aqueous solutions.

\subsubsection{Electrokinetic transport in micro- and nanometer-sized ge- ometries}

Electrokinetic transport refers to the combined effects of conduction, advection, and diffusion of dissolved ionic species and the fluid in which it is dissolved subject to some combination of thermodynamic forces. Since typical velocities of charged particles are low, 
the effects of magnetic induction are ignored; hence the subject is sometimes called 'electrohydrodynamics'; otherwise it is somewhat physically and mathematically analogous to the (mean-field) study of coupled transport in plasmas. When the effects of fluid flow are neglected, the subject is often referred to as electro-diffusion. In this case, the resulting mathematical description in a continuum approximation is similar to the classical conduction and diffusion of mobile charge-carriers through solids. It is then not too surprising that many of the non-linear transport-modulating behaviors seen in gaseous thermionic-emission devices and solid-state semiconducting devices, i.e. rectification of current flow and gating/valving functions, may also be exhibited by electrokinetic "fluid state" devices, albeit with some differences.

\section{Boundary layers and boundary-layer effects}

It is well-known that the interface between two phases acquires a boundary layer in order to achieve thermodynamic equilibrium [32]. The boundary layer will be discussed in more detail in Chapter 1, but a brief introduction is given here. In aqueous electrolytic solutionsor any system with mobile charge- the boundary region typically involves a small-scale separation of charge. Thermodynamically speaking, this accommodates the equilibrium of the charged species, ensuring that the electrochemical potential of each is continuous across any (arbitrary) boundary between the phases and can be thought of as the result of a competition between diffusive thickening and electrostatic attraction.

If a bulk aqueous electrolyte is in contact with a solid, the boundary layer extends into the liquid 10's to 100's of nanometers and is known as the electric double layer (EDL) or Debye layer. Under normal circumstances, most of the liquid is bulk-like in the sense that it maintains local electro-neutrality (LEN), and these thin layers exhibit strong charge separation which effectively shields the surface charge present in the solid-side of the boundary. The unbalanced charge in these layers is responsible for a number of the electrokinetic effects critical to micro and nanofluidic device operation. In microfluidic channels with tangentially applied E-fields, charges in the Debye layer provide a boundary-layer electric body force which effectively drags the bulk fluid along via viscous coupling, which can eventually result 
in a steady or quasi-steady electro-osmotic flow (EOF) with a uniform, plug-like flow profile in hydrodynamically unimpeded channels. In channels with nanometer dimensions, EDL's from adjacent walls may overlap, with strong effects on both species transport and fluid flow through the channel. When strong fields and concentration gradients are present, a number of interesting effects appear which are related to the distortion of these boundary layers and incipient electrokinetic instabilities [6, 68].

Strictly speaking, the conductive transport of ions and charged macromolecules, known as electrophoresis, is also dependent upon the structure of the Debye layers surrounding the particles. However, this is often accounted for in a more elementary way without much loss of generality, especially for small ions under moderate conditions. The Stokes drag and electric force on the particle compete to yield uniform motion in an applied field where an effective ionic charge and effective hydrodynamic radius can be used to define the electrophoretic mobility. The relationship between the actual values of the ionic radius (or molecular geometry for a more general charged macromolecule or colloidal particle) and the surface/ion charge must be taken into account for an accurate description, particularly under extreme circumstances [32]. The electrophoretic mobility $\mu$ is typically assumed to obey the Einstein relation,

$$
D=\frac{\mu k T}{q}
$$

where $D$ is the (self) diffusivity in the limit of infinite dilution, $k$ Boltzmann's constant, $T$ the temperature and $q$ is the net charge. Thus, the two quantities are used interchangeably both here and in much of the literature.

Some of the details of these transport phenomena relevant to the remainder of the dissertation are given in the following chapter. The above discussion is intended to give a brief introduction and to make the impression that devices containing both micrometer-sized and nanometer-sized channels operating display a number of interesting effects. This dissertation focuses primarily on novel electro-diffusive effects associated with ion transport across so-called perm- or charge- selective interfaces, which tend to favor transport of one sign of ion over the other. Other important effects, which are considered briefly in the last chapter, involve the coupling between the ionic distributions, electric field, and fluid flow. 


\subsection{The NCM/NMI device}

\subsubsection{Device construction, experimental details, etc.}

The NCM/NMI experimental devices (see Figure 1.1 or Ch. 3 Figure 4.1) consist of a roughly $1 \mathrm{~cm}$ long microchannel etched into a glass slide with a macroscopic reservoir and electrode at each end of the device. The channel is approximately trapezoidal in cross-section, with the channel depth being on the order of 10's of microns, and the width on the order of 100's of microns. The glass walls acquire a negative surface charge in aqueous solution at $\mathrm{pH}$ 7, which supports EOF. The left reservoir is directly attached to the channel and the right reservoir is separated from the channel by the nanocapillary membrane, schematically represented in Figure 1.1 by a smaller channel. The left reservoir is held at ground and the right reservoir biased during experiments.

\subsubsection{On- and Off-state Illustrations}

The NCM/NMI device has two distinct modes of operation corresponding to whether or not the right reservoir is biased positive or negative with respect to ground [37, 24]. The pores in the nanocapillary membranes are about $10 \mathrm{~nm}$ in diameter and the walls have a fixed negative surface charge under typical experimental conditions. The Debye layers in the pores are thick enough that they effectively overlap, blocking the passage of a significant fraction of the co-ions, i.e. those ions with the same sign as the surface charge (anions in this case,) which would normally be present in the total ionic flux. This condition is known as perm-selectivity, or equivalently, charge selectivity, and will be discussed in greater detail in the next chapter. Here it is sufficient to note that charge selectivity results in a flux imbalance across the membrane which drives the development of depleted and enriched regions on either side of the membrane. A conceptual model, due to $\mathrm{Pu}$ [43], can be used to understand this phenomenon, known as concentration polarization $(\mathrm{CP})$. The essential 
(a)

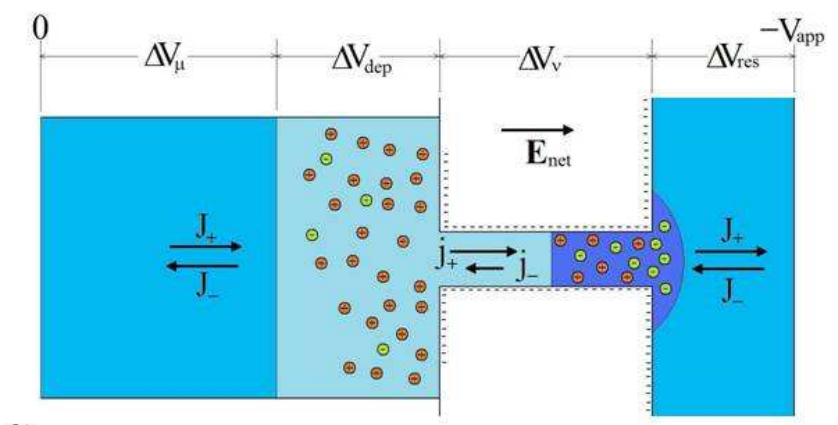

(c) Anion imb alance condition:

$J_{-} A_{\mu}=\int_{\text {res }} J_{-} d A_{\text {res }}>j_{-} A_{\text {nano }}$

Cation balance condition:

(b)

$J_{+} A_{\mu}=j_{+} A_{\text {nano }}=\int_{\text {res }} J_{+} d A_{\text {res }}$

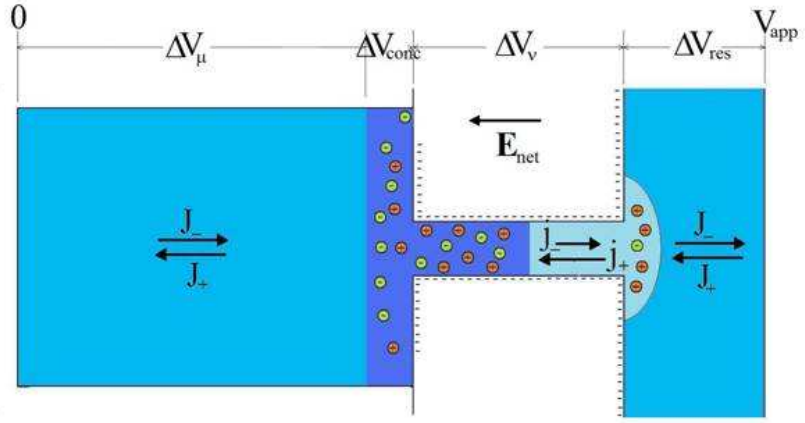

Bulk electron eutr ality:

$$
J_{+}=-J_{-}
$$

Nanochannel permselectivty for fixed

negative surf ace charge:

$j_{+}=-\alpha j_{-} \quad \alpha \geq 1$

Figure 1.1: Schematic On- and Off- state device illustrations showing the basic relationship between species flux densities $j_{ \pm}$, i.e. number of particles per unit area per unit time, and salt flux density, $J_{+} \equiv\left(j_{+}+j_{-}\right) / 2$, and charge flux density, $J_{-} \equiv\left(j_{+}-j_{-}\right) / 2$ for a twospecies system. The depleted and enriched regions are indicated respectively by lighter and darker shades of blue.

ingredients are shown in Figure 1.1. The resulting layers are known by a variety of names in the literature including Nernst layers, diffusion layers (DL's), and concentration polarization layers (CPL's). Here, we loosely follow the terminology and taxonomy formally laid out in Ref. [68]. The term 'CPL' will generally refer to the collective structure while 'DL' will refer to an individual layer.

As shown in Figure 1.1, the depleted DL for a negatively charged membrane forms on the side of the membrane/nanochannel closest to the anode and the enriched DL forms closest to the cathode, e.g. the electric field drives anions (co-ions with respect to the membrane charge) out of the depleted DL and into the enriched DL. Since the system naturally attempts to maintain electro-neutrality over large regions, this results in the respective de- 
pletion and accumulation of both ions. However, it is important to note that at sufficiently high currents, the system can no longer maintain local electro-neutrality and a macroscopic charge-separation occurs (in contrast to the microscopic separation of charge in the Debye layers.) This results in a number of interesting effects.

\subsection{NCM/NMI applications and experiments}

The NCM/NMI device is representative of a wide class of sample pre-concentration devices $[61,16,11,26,41,15,25]$. These devices employ either so-called classical (i.e. locally electro-neutral) CPL effects, or effects due to the extended space charge and electrokinetic instabilities occurring at higher-voltages/currents. Both types of concentration mechanism can be employed in the NCM/NMI depending on the device bias. In the on-state (forward bias) mode, classical CPL effects create a locally electro-neutral enriched DL in the microchannel adjacent to the membrane. A similar phenomenon is shown in Figure 1.2 for a device with a V-shaped microchannel connected to a reservoir by a single nanochannel. The off-state concentration mechanisms are much more difficult to understand and may be a result of a complex of effects which depend on the appearance of extended space charge.

While the NCM/NMI is intended for sample pre-concentration, it also functions as a fluidic rectifier, permitting a higher electric current in the on-state than the off-state, hence the naming convention. The concentration mechanism in the on-state is directly related to the function of the device as a fluidic rectifier. As can be anticipated from the results in Chapter 2 (see Ch. 2 Figure 3.8), the enriched concentration profile in the microchannel DL increases along with the rectification strength of the device. While the models used in Chapter 2 do not have any direct predictive bearing on the off-state concentration effects, they may aid in understanding the origins of these effects. Because the perm-selective membrane acts as a barrier to electro-neutral diffusion, and further, since the microchannel restricts convective mixing, the device also exhibits strong hysteresis effects when cycled between the off- and on-states. While the actual sample concentration process is not a central topic in this dissertation, a deeper understanding of the fluidic rectification and hysteresis effects is expected to help guide future work on the concentration process. Indeed, these results have already 


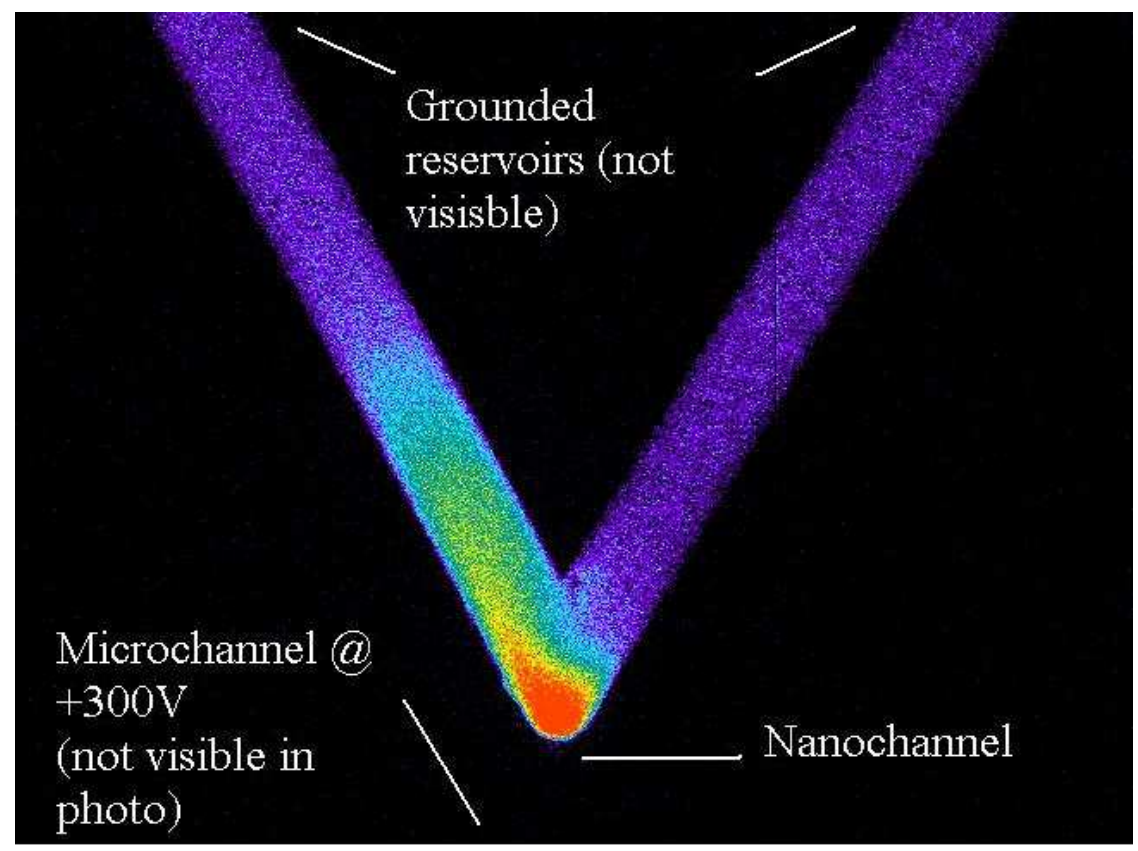

Figure 1.2: Fluorescent microscope image of dye concentrated in the on-state in a V-channel device with a single nanochannel as the perm-selective element. The device operation is essentially the same as that in the NCM/NMI on-state. Image courtesy of K. Kelly (unpublished.)

been used to conduct some simple studies into steady-state concentration polarization of 3-species systems [7].

\subsection{Overview of dissertation}

A significant portion of the dissertation is devoted to understanding locally electro-neutral electro-diffusive contributions to the rectification effect while ignoring complications due to EOF and space-charge. However, the expected effects of these complications are discussed and some effort is made to extend the theory.

Chapter 2 presents an overview of background material and notes on the modeling approach employed in the remainder of the text. The aim is to provide some necessary details and place the results obtained in the rest of the dissertation in the broader context of the subject of electrokinetics. Chapter 3 contains the central results of the dissertation. A survey of fluidic rectification is presented, along with a general explanation of the fundamentals, 
different types of device, and some historical perspective. Then several variations of a simple model are used to understand fluidic rectification in greater detail and some qualitative predictions are made.

Chapter 4 contains a study of experimental results, primarily a discussion of the observed fluidic rectification behaviors in the context of the models of Chapter 3. The hysteresis, or so-called 'chemical ratchet' effect observed in the NCM/NMI device is explained in terms of the models of Chapter 3. There is also a brief discussion of the off-state concentration process. The collective observations, both successes and failures of the models of Chapter 3 , along with the consideration of some of the features in the off-state concentration process are then used to motivate both future experiments, discussed at the end of Chapter 4, as well as to provide some observations relevant to the work in Chapter 5.

The dissertation concludes with Chapter 5, where the first steps in the extension of the simple theories of Chapter 3 are taken. While most of the work in Chapter 5 is preliminary, one interesting result is that the membrane is shown to become polarized under passage of an electric current. This effect complements the polarization of the double-layer, which is known to develop into an extended, macroscopic space charge. This membrane polarization may help to explain how this excess space charge originates in realistic systems with fixed numbers of ions while maintaining global electro-neutrality. This issue has not, to the author's knowledge, been addressed in any of the previously published works on the development of the extended space charge. To conclude, the results and methods of Ref. [51] are applied to the case of an electrolyte adjacent to an ideal membrane confined to a narrow 2D channel. As might be expected, it is shown that there exists a minimum allowed wavenumber for perturbations corresponding to a maximum wavelength. 


\section{Chapter 2}

\section{Background theory and related problems}

\subsection{Chapter introduction and overview}

In this chapter, the basic electrokinetic transport mechanisms and the corresponding mean-field governing equations are reviewed in dimensional and non-dimensional form. This is followed by a discussion of the choice of scales for the various physical quantities and resulting dimensionless parameters that characterize the problem. Then the structure, terminology and properties of the electric double-layers are introduced in terms of the equilibrium Gouy-Chapman model and the Poisson-Boltzmann equation. Charge-selective transport due to overlapping EDL's is then considered in the context of cylindrical nanochannels, along with a brief comparison to membrane models. The non-equilibrium $I-V$ response and the development of concentration polarization are then discussed along with some fundamental open problems in the field.

\subsection{Continuum models of electrokinetics}

A common starting point for modeling of electrokinetic phenomena in micro- and nanofluidic systems is to assume a continuum, mean-field description of the system. This neglects a number of complications by assuming the solvent is a structure-less, incompressible, dielec- 
tric liquid. Also, the ions are be treated in a "smeared-out" fashion, effectively as though they compose a simple, charged fluid. It is generally assumed (unless appropriate reactions are taken into account) that the ions are those of a strong electrolyte, yielding stoichiometrically consistent concentrations regardless of the possibility of $\mathrm{pH}$ shifts, etc. The finite size of all molecules and ions is neglected -which will be of the greatest consequence near the solid-liquid interface- and the transport properties, specifically the diffusivity, is assumed to be the same as in the limit of infinite dilution. Some comments regarding the consequences of these simplifications are made in the course of discussion.

\subsubsection{The governing equations}

For dissolved ionic species $i=1, \ldots, M$ subject to gradients in electric potential, concentration, and pressure, the net flux density (particles per unit area per unit time) of the $i^{\text {th }}$ species is given by the Nernst-Planck flux,

$$
\tilde{\mathbf{j}}_{i}=\tilde{\mathbf{v}} n_{i}-D_{i}\left(\tilde{\nabla} n_{i}+\frac{q_{i}}{k T} \tilde{\nabla} \phi\right)
$$

The first term above is the advective contribution to the flux, and the term in parentheses the combined electro-diffusive flux, where the fluid velocity is $\tilde{\mathbf{v}}$, the (dimensional ${ }^{1}$ ) number density of the $i$ th species is $n_{i}$, the diffusivity $D_{i}$, ionic charge $q_{i}$, and the electric potential $\Phi$. Combining this with the requirement of conservation of mass, i.e. the continuity equation, gives the conduction-diffusion-advection equation,

$$
\frac{\partial \tilde{n_{i}}}{\partial \tilde{t}}+\tilde{\mathbf{v}} \cdot \nabla \tilde{n_{i}}=\nabla\left[D_{i}\left(\nabla \tilde{n_{i}}+\frac{q_{i}}{k T} \tilde{n_{i}} \nabla \Phi\right)\right]
$$

The total electric potential is given by the Poisson equation,

$$
\nabla^{2} \Phi=-\frac{1}{\epsilon \epsilon_{0}} \sum_{i=1}^{M} q_{i} n_{i}+\tilde{N}(\tilde{\mathbf{r}})
$$

\footnotetext{
${ }^{1}$ dimensional quantities are denoted by a " , wherever the same symbol is used for the dimensional and dimensionless quantity.
} 
where $\tilde{N}(\tilde{\mathbf{r}})$ represents any fixed charge density present in the system.

Fluid flow is governed by the Navier-Stokes equation,

$$
\frac{\partial \tilde{\mathbf{v}}}{\partial t}+(\tilde{\mathbf{v}} \cdot \nabla) \tilde{\mathbf{v}}=\frac{\rho_{e}}{\rho} \nabla \Phi+\frac{\eta}{\rho} \nabla^{2} \tilde{\mathbf{v}}-\frac{1}{\rho} \nabla \tilde{p}
$$

subject to the following condition for incompressible flows,

$$
\nabla \cdot \tilde{\mathbf{v}}=0
$$

Here, $\rho_{e}$ is just the total charge density from the Poisson equation, $\rho$ the mass density, and $\eta$ the dynamic viscosity. Fluid properties are usually assumed to be equal to that of the pure, bulk solvent.

In most microfluidics applications, the second, so-called inertial, term on the left-hand side, $(\tilde{\mathbf{v}} \cdot \nabla) \tilde{\mathbf{v}}$ is usually neglected for reasons discussed in the following sub-section. Temperature differences and the transport and dissipation of heat are typically ignored. For sufficiently low current densities, where Joule heating is negligible, this is a safe assumption. Often there is no applied external pressure, and this term is omitted. Whether or not this is justifiable in all systems, particularly when there is strong cross-coupling between transport mechanisms, is questionable [36, 4]. For moderate field strengths and concentration gradients the bulk-like portions of the electrolyte will be able to maintain (quasi) local electro-neutrality (LEN) and the only appreciable electric body forces arise in the boundary layers, as in the case of classical EOF as discussed in the introduction. In this case, the body force term is omitted from the equation for bulk and replaced by an appropriate slip condition at the boundary. At sufficiently high voltages/currents and/or concentration (conductivity) gradients, this is not the case; local electro-neutrality may be violated over scales larger than the Debye length near solid-liquid boundaries. These situations will be discussed somewhat later on when relevant.

\subsubsection{Dimensionless forms and important parameters}

As is often the case with nonlinear systems, the equations are put into dimensionless form, in large part for mathematical convenience in finding physically appropriate approxi- 
mations. However, since this typically results in the identification of important dimensionless parameters that characterize the system, it also leads to some clarity and physical insight. In this section, several important parameters are introduced and discussed along with the appropriate non-dimensional forms of the equations.

\section{Dimensionless forms}

The scaling convention here is similar to that in Ref. [68]. The equations governing species concentration are written

$$
\frac{\partial n_{i}}{\partial t}+\operatorname{Pev} \cdot \nabla n_{i}=\nabla\left[\mathcal{D}_{i}\left(\nabla n_{i}+z_{i} n_{i} \nabla \phi\right)\right] .
$$

where $P e$ is the Peclet number. The non-dimensional Poisson equation is given by

$$
\varepsilon^{2} \nabla^{2} \varphi=-\sum_{i=1}^{M} z_{i} n_{i}+N(\mathbf{r}),
$$

where $\varepsilon$ is the dimensionless Debye length Lastly, neglecting the inertial terms, the dimensionless Navier-Stokes equation is written,

$$
\frac{1}{S c} \frac{\partial \mathbf{v}}{\partial t}=\frac{\rho_{e}}{\rho} \nabla \phi+\frac{\eta}{\rho} \nabla^{2} \tilde{\mathbf{v}}-\frac{1}{\rho} \nabla \tilde{p}
$$

with the Schmidt number Sc.

These equations, along with a non-dimensional incompressibility condition, must be supplemented by appropriate boundary conditions. Generally, the body-force term in the Navier-Stokes equation can be ignored and the body-force acting in the electric double layers included by means of a non-zero slip velocity at the solid-liquid interface for the case of very thin EDLs. This yields the electro-osmotic flow, as discussed in the Introduction. It is also known as electro-osmosis of the first kind, to distinguish it from convective motions that can arise due to modified (i.e. polarized) Debye layers, or due to instabilities in these layers. Other boundary conditions will be discussed further in the course of studying a specific problem.

The physical meanings, relationships to the (implied) approximations, and typical magnitudes of the dimensionless parameters $P e, \varepsilon$, and $S c$ are discussed subsequently. However, 
the issue of fundamental scales warrants some discussion first.

\section{Choice of scaling for variables}

There is some latitude in choosing the appropriate scales for the non-dimensional forms. While the choice is to some extent arbitrary for linear equations, the scales should be chosen to reflect characteristics of the phenomena under consideration. For non-linear equations, the choice of scale will affect the ability to resolve boundary layers in analytic approximations, and-for numerical work- can affect the convergence and stability of the solution. There are several apparent scales to choose from for the quantities appearing in the electrokinetic governing equations. Some choices are less contentious than others.

The species concentrations are scaled by the bulk concentration of one of the components (which are the same in a symmetric binary system), so that the dimensionless concentration is

$$
n_{i}=\frac{\tilde{n}_{i}}{n_{o}}
$$

In multi-component systems, the diffusivity may be scaled by that of one of the components, or by some combination, average, etc. of the individual diffusivities. In any case, the choice will be specified for a particular problem when necessary and the typical value will be denoted as $D_{o}$. Thus the dimensional diffusivity for the $i^{t h}$ component given by $D_{i}=D_{o} \mathcal{D}_{i}$. For the problems considered here, the electrolyte is assumed symmetric and binary, so that both species have the same diffusivity, so the dimensionless value in the open electrolyte ${ }^{2}$ is then just $\mathcal{D}=1$. In these cases, the diffusivity $\mathcal{D}$ is usually dropped from the equations. The potential is scaled according to the thermal voltage, $\Phi=V_{t h} \phi$ where $V_{t h}=k T / e=25.4 \mathrm{mV}$. Some authors [6,29] also scale by the applied potential.

The dimensionless time is written

$$
t=\frac{\tilde{t} D_{o}}{L^{2}}
$$

\footnotetext{
${ }^{2}$ As opposed to electrolyte confined to nanopores or that infiltrating a polymer gel, where some effective diffusivity different from unity will be assumed.
} 
Note that the time-scale chosen corresponds to the characteristic relaxation time for locally electro-neutral diffusion over an as-of-yet unspecified length scale $L$. There are shorter timescales associated with the relaxation of the Debye layer (at all voltages) and intermediate time-scales associated with the development of extended space charge occurring at high currents $[1,52]$. Again, it is not a priori apparent which scale is the most appropriate, but since the bulk of the work here will be concerned with systems that can be described by locally electro-neutral (LEN) electro-diffusion, or the stability of quiescent (LEN) electro-diffusion subject to perturbations, this choice seems reasonable. However, there is still some ambiguity in that an appropriate length scale has yet to be specified.

The length scale $L$ appearing in these definitions is usually assumed to correspond to the typical length scale over which the (LEN) concentration varies, usually on the order of the distance between electrodes. However, it is not clear that this is always the most appropriate choice. With the NCM/NMI, several natural length scales are present. Which one to choose depends on the particular phenomena of interest and also upon the duration of observation/measurement relative to the dominant intrinsic time scale. For instance, in consideration of problems regarding the double-layer overlap inside of the individual nano-capillaries, an appropriate length scale is the capillary radius (on the order of 10's of nanometers) and the intrinsic time-scale would be that corresponding to the growth of these double-layers, i.e the Debye time $t_{\lambda}=\lambda_{D}^{2} / D_{o}$ or about $10^{-7}$ seconds ${ }^{3}$. Outside of the membrane and further restricting attention to electro-neutral phenomena, i.e. for low currents and times much longer than the Debye time the various widths and lengths of the system present other choices.

If the problem may be regarded as essentially $1 \mathrm{D}$-as will be done for the majority of the work here-then the typical membrane-to-electrode distances $L_{E}$ are obvious candidates. However, so is the membrane thickness $\delta$. The best choice for the scale is the length over which the concentration changes from a bulk value, i.e. the value held near the entrances to large reservoirs, to the interfacial value near the membrane(channel)-electrolyte interface. However, in the case of devices with centimeter-long microchannels, the full development of so-called diffusion layers of this length requires times on the order of $10^{4}$ sec. (see Ch. 2

\footnotetext{
${ }^{3}$ where $\lambda_{D}$ is the dimensional Debye length, to be discussed shortly
} 
Table 3.1.) In such cases, during most experiments, this will not be a fixed quantity. For most of the calculations here (specifically, those assuming a quasi-steady state) the length scale will be set by the membrane thickness. There is more to be said regarding the best choice of time-scale.

The intrinsic velocity and pressure scales are chosen by considering steady-state forcebalancing of the Navier-Stokes equations. There are some subtleties regarding the omission of the inertial terms and some discussions suggest this is not appropriate [6, 29]. However, in performing a true steady-state force-balance of the dimensional form of the Navier-Stokes equation, these do not matter. Consider that, by definition, a true steady-state of the dynamical variable $a$ is given by the condition $\dot{a}=0$, i.e. the total time-derivative is zero. For the fluid velocity, steady-state implies that

$$
\dot{\tilde{\mathbf{v}}}=\frac{\partial \mathbf{v}}{\partial t}+(\tilde{\mathbf{v}} \cdot \nabla) \tilde{\mathbf{v}}=0
$$

Thus, the force-balance does not depend on the vanishing of the inertial terms.

By assuming no applied pressure gradient, the natural velocity scale that arises is the so-called electroviscous velocity,

$$
v_{o}=\frac{\epsilon \epsilon_{o} V_{t h}^{2}}{\eta L}
$$

Note that some scaling arguments appeal to velocities on the order of magnitude of typical electro-osmotic velocities, i.e. about $1 \mathrm{~cm} / \mathrm{s}$. These may be appropriate when considering systems dominated by externally driven flows, e.g. those due to electro -osmosis of the first kind. But when considering stability of the quiescent electro-diffusion problem, the intrinsic electroviscous scale is the correct scale.

If instead, the electric body force is assumed to be zero (i.e. no applied electric field,) the force balance yields a scaling for the pressure,

$$
p_{o}=\frac{\eta v_{o}}{L}
$$

From these fundamental scales, several dimensionless numbers arise. They will be introduced and discussed below. 


\section{Important dimensionless parameters}

Three dimensionless parameters appear in the governing equations. However, given the nature of the approximations made along with the typical justification, it is prudent to discuss four:

- Debye parameter/length

- Peclet number

- Reynolds number

- Schmidt number

The first parameter arises from the Poisson equation. The Debye parameter is a dimensionless parameter often called the dimensionless Debye length, $\varepsilon=\lambda_{D} / L$ where the Debye length is

$$
\lambda_{D}=\sqrt{\frac{\epsilon \epsilon_{o} k T}{e^{2} n_{o}}},
$$

and the length scale is $L$. Physically, it is a measure of the extent of the Debye boundary layers, a.k.a. the electric double layers. For a bulk concentration of $10 \mathrm{mM}$, the bulk number density is about $n_{o}=6 \times 10^{24} \mathrm{~m}^{-3}$ and the Debye length is about $4.8 \mathrm{~nm}$.

The Peclet number, $P e=v_{o} l / D_{o}$, which is essentially a ratio of convective to diffusive transport can and has been defined a number of ways, leading to several different estimates. Scaling by a typical electro-osmotic velocity and considering the various length-scales, from $10 \mu \mathrm{m}$ to $0.01 \mathrm{~m}$, Peclet numbers range from $10^{2}$ to $10^{5}$. By using the electroviscous velocity scale the Peclet number estimate is independent of length,

$$
P e=\frac{\epsilon \epsilon_{o} V_{t h}^{2}}{\eta D_{o}} .
$$

Thus, for a typical aqueous electrolyte, $\eta=10^{-3} \mathrm{Nsm}^{-} 3$ and $D_{o}=10^{-9} \mathrm{~m}^{2} \mathrm{~s}^{-1}$ (order of magnitude, $) \quad P e=2.248 \times 10^{-3}$. Estimates for this number have also been given based on considerations of finite ion radius and separation, thus also independent of system size; these yield instead $P e \simeq 0.5$ [50]. If the point of view is taken that the appropriate scale is chosen according to the dominant velocity contribution in the system at hand, then it seems 
appropriate that the Peclet number for systems dominated by EOF differs from the Peclet number for systems dominated by electro-diffusion with internal electro-convection above a threshold. This point may be important in explaining some experimental observations in Chapter 3.

The Reynolds number is a ratio of momentum transport by convection to momentum transport by viscous dissipation, $R e=v_{o} L \rho / \eta$. In introducing the approximate NavierStokes equation above, the fact that the Reynolds number in microfluidic systems is typically $R e \ll 1$ has been used to linearize the full equation with respect to perturbations of fluid velocity allowing the omission of the term $(\tilde{\mathbf{v}} \cdot \nabla) \tilde{\mathbf{v}}$. Following from the previous discussion, it can be anticipated that the Reynolds number may be evaluated in a number of ways. In an EOF-dominated ${ }^{4}$ microfluidic system, the typical fluid velocity is on the order of $\mathrm{cm} / \mathrm{s}$ and the typical linear dimension on the order of $10^{-4} \mathrm{~cm}$. If we assume the kinematic viscosity of the solution to be approximately the same as the solute, generally water, with a value $\nu=\eta / \rho \approx 1 \times 10^{-2} \mathrm{~cm}^{2} / \mathrm{s}$ at room temperature, this gives $R e=10^{-2}$. If instead the applied voltage and electroviscous velocity scale are used, $R e \sim 100$ [29]. This can be expected to alter the value for the Peclet number as well. For the thermal voltage scale and electroviscous velocity, one obtains

$$
R e=\frac{\epsilon \epsilon_{o} V_{t h}^{2}}{\eta \nu}
$$

where $\nu=\eta / \rho$ is the kinematic viscosity, $=10^{-6} \mathrm{~m}^{2} \mathrm{~s}^{-1}$. This gives $R e=2.2 \times 10^{-6}$.

Lastly, the Schmidt number may be introduced as the ratio of Peclet and Reynolds numbers,

$$
S c=\frac{P e}{R e}=\frac{\nu}{D_{o}}
$$

Physically, the Schmidt number, like the Peclet number, may be interpreted as a measure of viscous to diffusive transport of momentum but is strictly a property of the fluid, rather than the flow [42]. However, this begs an important question: If the Schmidt number is a property of the fluid, shouldn't the diffusivity appearing in its definition include in some way

\footnotetext{
${ }^{4}$ In the sense that the fluid flow is primarily due to microchannel EOF of the first kind.
} 
the self-diffusivity of the solvent molecules instead of only the solute?

\section{Summary: Why scaling is important}

The point of all the preceding, and inconclusive, discussion of scaling is simply that there are many possible relevant scales; which ones to choose are not automatically clear and the literature on electrokinetics offers little in the way of concrete guidance. To re-iterate and re-state, both theory and simulation rely on proper choice of scaling to reliably resolve the phenomena of interest. In this dissertation, the question does not need to be answered completely; the instability calculations and work towards threshold estimates in Chapter 4 which do rely on the choice of scale are only preliminary calculations and no directly testable predictions are made for which this should matter. The theoretical and numerical studies in the references use various scalings and all predict apparently related phenomena, namely vortices and other electro-kinetically driven, often unstable, hydrodynamic responses. However, these occur under a variety of conditions and for fundamentally different electrokinetic systems. Later, in the course of studying experiments in Chapter 3 and the first sections of Chapter 4, it becomes clear that the NCM/NMI displays a number of features reminiscent of many of these instabilities. It seems that one must take the issue of scale-choice very seriously in order to make any real progress on understanding instabilities in these systems. In particular, a predictive theory of diffusion-layer/depletion length selection will require a careful consideration of these issues and the choice is likely to be highly device-dependent, i.e. is the system dominated by EOF of the first kind or internal electroviscous velocity? What is the appropriate model for the Peclet and Schmidt numbers, i.e. should solvent self-diffusivity be accounted for, particularly with the Schmidt number? 


\subsection{The electric double layer}

\subsubsection{Thermodynamic basis for boundary layers}

The importance of interfacial phenomena in physical systems is underscored by the ubiquity of boundary layers occurring at interfaces between any two distinct phases as a result of relaxation to equilibrium. This manifests itself, for instance, in solid-state physics at the interface between two different crystalline materials. The layers on either side of the interface must be stretched or compressed with respect to the bulk phase of the pure material so that the new lattice spacings on either side of the interface reflect a mechanical equilibrium between the (now deformed) lattices of the constituent phases. When these conditions cannot be met by relatively small re-arrangements about the original equilibria of the substances, more drastic measures are taken; defects may form and propagate, localized phase changes may occur, and/or instabilities may develop. Thus the structure of the interface is altered even more dramatically, or perhaps even loses its integrity altogether. In addition to purely mechanical mechanisms, chemical and electrical equilibria must also be reached, and accordingly the composition and bonding at the interface must re-arrange somewhat as well. Similar layers occur in fluid and plasma physics at the interfaces between the fluid or

plasma and the container walls, as well as between regions with distinctly different properties. Generally speaking, unless the scale of these layers is similar to the scale of other important physical features in the systems, they may be treated as boundary-layers, or even ignored completely in some cases.

These double-layers exist outside of equilibrium as well, sometimes undergoing radical structural changes, but often retaining some aspects of the equilibrium layers. Ignoring the mechanical aspect, solid state physics supplies yet another (and more relevant) example; electrical interfacial layers form across junctions between materials with different carrier densities (i.e. conductivities.) These layers persist under applied fields and in macroscopic, room-temperature devices, they may be described using similar electro-diffusive models (mi- 


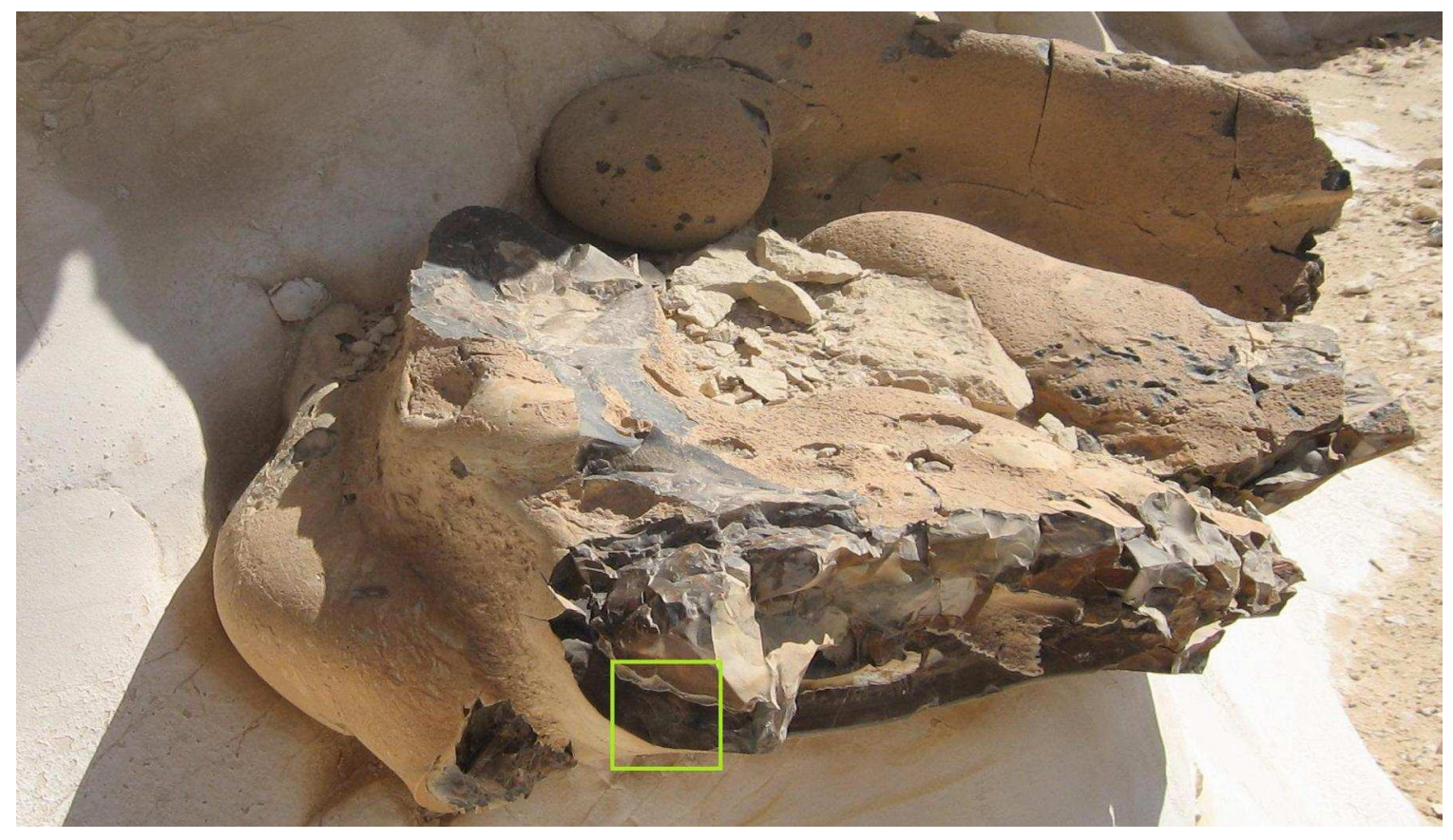

Figure 2.1: Thermo-mechanical double-layers (green box) formed by chemical and physical relaxation at the boundary between basaltic lava and the surrounding limestone, resulting in a macroscopic region - the darker flint-like material- of different appearance and composition than either of the two 'bulk' materials. Similar features can be found in many of the rock formations of the Negev desert. 
nus any analogue to convective flow) to those employed here to study electrokinetic phenomena. As seen in the description of the NCM and Nafion I-V curves in the preceding section, many of the same non-linear response phenomena exhibited in semi-conductor junction devices such as carrier depletion, the accompanying limiting current behavior, and non-ohmic current-voltage response are observed in electrokinetic systems.

In non-equilibrium situations, these layers may exert a strong influence on the behavior of the bulk phases, i.e. on the net transport of material or momentum through the system in question. There exist regimes in which the properties of the double-layer are largely un-affected by the conditions on the bulk and likewise other regimes in which the bulk conditions and the structure of the double-layers are not independent. As the length scale of the systems approaches that of the double-layers, this situation becomes acute in the sense that it is difficult, if not impossible, to divide the system neatly into boundary-layers and bulk phases. Thus, on the scale of these layers, the distinction is seen to be largely one of convenience and somewhat semantic in nature. For electro-kinetic systems, the discussion of the distinction between the bulk-like phases and the boundary region can be facilitated by the introduction of the so-called Gibbs dividing plane, an artificial boundary across which the net electro-chemical potentials of each species $\mu_{i}=\ln n_{i}+q_{i} \Phi$ are assumed continuous but where the concentrations and electric potentials may change from one phase to another. In equilibrium, the gradient of the electrochemical potentials is zero so there is no species flux. However, the concentration and electric potential change from the bulk value of phase 1 to the bulk value of phase 2 across the boundary region. When the layers are thin and quasi-equilibrium conditions may be assumed to hold (in the sense that the boundary layer structure does not deviate appreciably from its equilibrium configuration) the discontinuities in the electric potential and concentrations across the Gibbs plane and boundary region may be included in the problem by imposing continuity of the electrochemical potentials between phases across an infinitesimally thin boundary. This idea is employed to provide boundary conditions at the membrane-electrolyte boundaries in Chapter 2. 


\subsubsection{Equilibrium electric double-layers: Poisson-Boltzmann equa- tion and the Gouy-Chapman theory of the electric double- layer}

As a general physical principle, there is a separation of charge at the interface between two distinct phases $[17,32]$. While a great body of work is devoted to both general thermodynamic discussions of this phenomenon as well as specific and detailed treatments of the structure of the electric double-layer at the solution-solid interface, we will be content with a brief overview of one of the simplest-yet highly successful- versions of the theory, the GouyChapman picture. Here it is assumed that, by some mechanism, a charge is embedded in the solid side of the interface which attracts a predominance of counter-charge in a boundary layer of the solution nearest the surface (and necessarily implies a deficit of co-charge in this region.) Since the electrolyte is at some finite temperature, the associated thermal kinetic energy of the molecules prevents them from accumulating on top of one another, and the resulting distribution of charge is of the form of a diffuse boundary layer with a thickness determined by the competition of thermal and electrostatic energy.

The charges are all assumed to be 'smeared out' continuum charges and may approach the surface infinitesimally close, and furthermore there is no competition for space on the surface between, say, solvent molecules, and charged ions and that the 'structure' of the solvent is essentially unchanged. In short, the picture ignores many important effects, particularly steric effects, often overestimating the ion densities nearest the wall. Nonetheless this constitutes a reasonable description for systems large enough that discrete effects may be ignored. Some corrections may enter due to finite-size effects of the ions and the discrete nature and location of surface groups, etc. The most significant of these is a capacitative contribution of the so-called Stern layer, and corrections often include a reduced or modified charge density close to the surface (within a nanometer or so).

As mentioned in the introductory chapter, electric double-layers play several important 


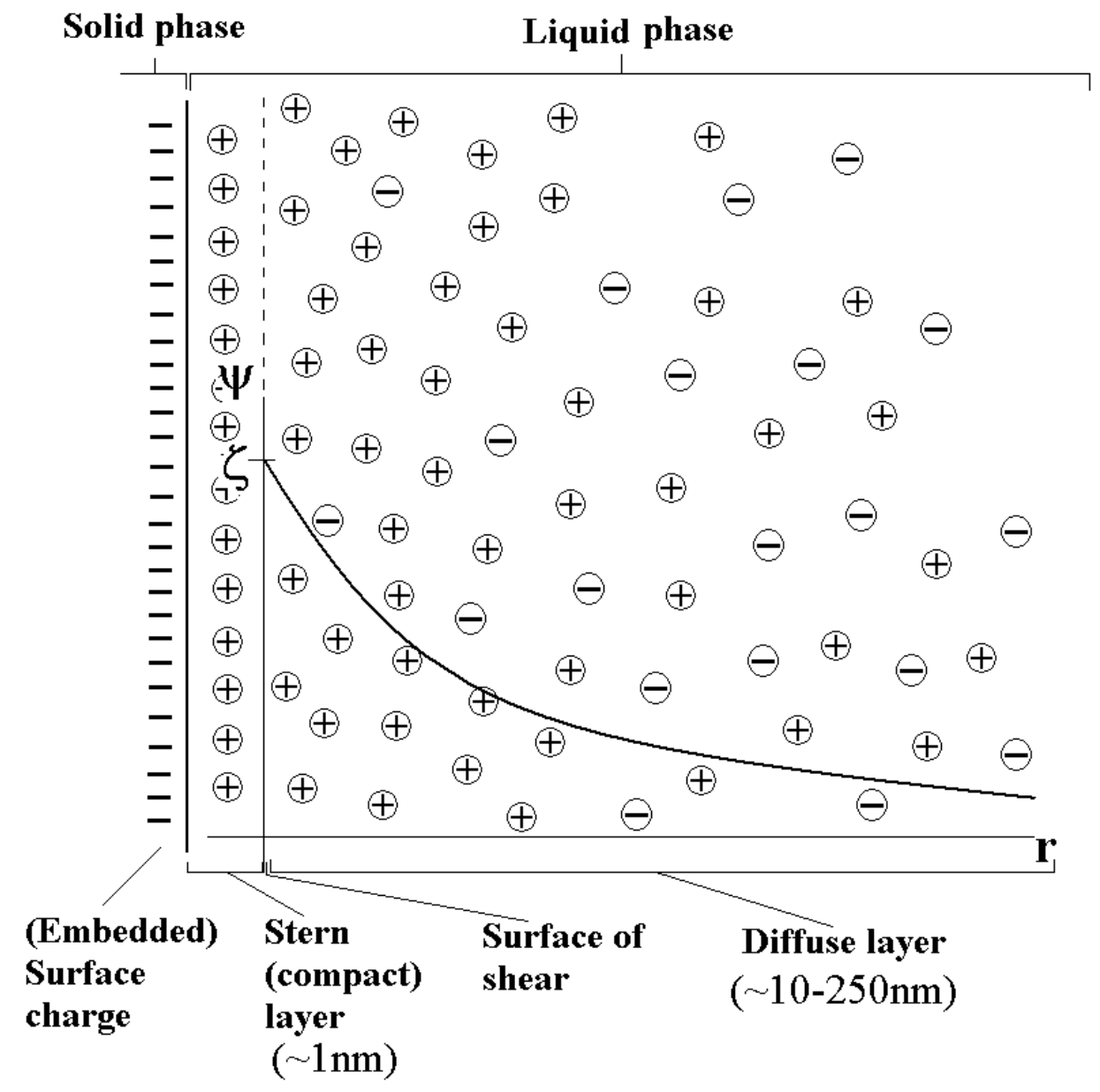

Figure 2.2: Schematic EDL anatomy showing various important features in the electric double-layer for a fixed negative surface charge, including the variation in potential from the so-called surface of shear which separates the compact, relatively immobile Stern layer from the diffuse portion of the double-layer. The zeta potential is defined as the value of the electric potential at this surface. This picture is fundamentally similar to the usual one appearing first in Ref. [17] 
roles in micro- and nanofluidic devices. They are responsible for generating EOF at the microchannel walls, i.e. what is known as EOF of the first kind, they provide the ionic atmosphere around dissolved ions and macromolecules, which affects the electrophoretic mobilities of these particles, and they serve as an intermediate region between bulk-like (locally electro-neutral) electrolyte and the ion profiles inside of membranes and nanochannels. Inside the nanochannels themselves, the overlap of the electric double-layers from adjacent walls is responsible for charge-selective transport, as will be discussed shortly. It has also been reported that the large electric body forces due to the relative thickness of the layers compared to the overall dimension of the channels can create a strong EOF response inside the channels of nanocapillary membranes [27]. While the channels considered here are at the lower bounds for the validity of this treatment $(a=5 \mathrm{~nm})$ this should be a sufficiently good approximation to make some general conclusions.

\section{The Poisson-Boltzmann equation}

Consider the electric double-layer in a sufficiently dilute aqueous electrolytic solution in contact with a material that has acquired (by means to be discussed shortly) a negative surface charge, for instance, the double-layer in a glass of salt water. For the time being, the ions due to dissociated water may be neglected, since these are typically negligible in number in comparison to ions in dilute solutions By definition, in equilibrium, the net generalized fluxes and forces are zero, implying the gradients of the corresponding potentials are zero, hence these potentials are spatially uniform. The electrostatic potential $\Phi$ in a 1-1 electrolytic solution, where the solvent is treated as a background continuum with dielectric constant $\epsilon$, is given by the Poisson equation,

$$
\nabla^{2} \Phi=-\frac{e}{\epsilon}\left(n_{+}(\vec{r})-n_{-}(\vec{r})\right)
$$

Here the number density (concentration) of each species is a function of position, and for the equilibrium problem, the bulk fluid is taken to be electrically neutral so that $n_{+}(\infty)=$ $n_{-}(\infty)=n_{o}$. The condition of thermodynamic equilibrium is given as

$$
\nabla \mu_{i}=0
$$


where $\mu_{i}$ is the electrochemical potential of the $i^{t h}$ species,

$$
\mu_{i}=q_{i} \Phi+k T \ln n_{i}
$$

Applying the equilibrium condition and integrating from some reference point where both electrostatic potential and concentration are known,

$$
n_{i}(\vec{r})=n_{\text {ref }} \exp \left(-q_{i}\left(\Phi(\vec{r})-\Phi_{r e f}\right) / k T\right)
$$

Typically, the reference point is taken deep inside the bulk where, as stated above, the concentrations of cations and anions are equal and the electrostatic potential is defined to be zero. In this case, setting $n_{r e f}=n_{o}$ and $\Phi_{r e f}=0$ and substituting the resulting expressions for the concentrations in the Poisson equations gives the so-called Poisson-Boltzmann equation for the electrostatic potential,

$$
\nabla^{2} \Phi=\frac{2 e n_{o}}{\epsilon} \sinh \frac{e \Phi}{k T}
$$

This equation governs the equilibrium electric potential in a solution of ions near a solid-liquid interface with either embedded surface charge, held at an applied potential, or both. It also holds reasonably well around equilibrium, particularly when the applied field is tangential to the structure, i.e. as in the typical microchannel or nanochannel (interior) walls. It has been used to estimate quasi-equilibrium transport properties (see Ref's [32, 18, 17, 54, 57, 10] and references therein for examples.) However, it is well-known that the double-layer may become polarized under even moderate applied fields [12] and this can yield dramatic effects when the fields/currents are perpendicular to the double-layer.

\section{Sources of surface charge}

Fixed surface charge at the solid-liquid interface can be attributed to a number of mechanisms [32], among those most important for micro-nanofluidic systems are the following:

- Surface charge due to dissociation of surface groups

- Surface charge due to specific adsorption of ions 
The first mechanism is most relevant for the microfluidic portion of the system. Typically, a functional surface site can lose or gain a proton (or, in principal, a hydroxide ion) to maintain chemical equilibrium with the adjacent solution. These changes happen fast enough relative to other dynamics in the system so as to consider them instantaneous. Since the relevant equilibrium generally depends on the population of hydrogen and hydroxide ions, it is a $\mathrm{pH}$ dependent effect. However, it has also been shown in a number of studies that both salt content and channel size can affect the distribution of surface charge $[54,57,10,2]$. The primary role of microchannel surface charge is to determine the strength of the EOF of the first kind. Changes in microchannel wall surface charge can also lead to non-uniform velocities. This could be employed to effect a mixing process, or detrimentally, it can cause abnormal (i.e. non-Taylor) broadening of samples.

Materials like the polycarbonate of the NCM membranes most likely acquire the majority of their surface charge through adsorption of negative ions. The details of the mechanism are beyond the scope of this dissertation and it generally considered is an un-resolved topic in the field. However, in a rough way, it may be understood as the result of two things; the hydrophobicity of the PC material and the relative size of anion hydration shells in comparison to cation hydration shells. It is energetically more favorable for the anions to shed this shell and attach to the surface than for cations. Surface group dissociation-binding (i.e. due to the introduction of functional surface groups by some treatment process) is not thought to play as important a role for materials like the PC-NCM. ${ }^{5}$ While it is also likely the case that these mechanisms are affected by the overall chemistry of the solution as well as likely the channel dimensions-particularly requiring consideration of surface-tension in small capillaries-the mechanisms have, to the author's knowledge, not been studied extensively.

\footnotetext{
${ }^{5}$ The content of this discussion from personal correspondence with Michael Hickner of Sandia Laboratories.
} 


\subsubsection{Permselectivity near equilibrium and simple models for the NCM}

The experiments analyzed in Chapter 3 have been conducted using two different types of membrane, Nafion and PC-NCM. Both are nanoporous membranes; they transport ions and fluid through numerous small pores of nanometer dimension. However, the nature of the pores, hence the characteristics, of the two types of membrane are markedly different. The NCM has relatively straight, uniform cylindrical pores at a controllable density. The pores have complete connectivity in the sense that a pore starting at one surface exits through the other. By comparison, the Nafion membranes have much more tortuous and irregular pores with poor connectivity, i.e. less than half of the pores connect the two surfaces [14]. Accordingly, one might anticipate that total currents are lower and hydraulic resistance much higher in the Nafion than the NCM; in fact, it is assumed here that no fluid flows through the Nafion at all. This assumption seems valid, but the same cannot be said of the NCM with as much certainty [27].

\section{Pore models vs. membrane models}

NCMs have characteristics like ion-exchange membranes [63], so using models like those used to study ion-exchange membranes $[46,49,50,51,68]$ is justified to some extent. However, NCMs can produce significant EOF, while ion-exchange membranes virtually eliminate it [27]. The position here is taken that, based on the similarity between ion-exchange membrane and track-etched membrane $I$ - $V$ characteristics that EOF is not critical to the general qualitative features of NCM $I-V$ response. It is, however, anticipated to have some effects. Since Nafion is intermediate between the track-etched NCMs with straight pores and complete connectivity, it assumed that the ion-exchange membrane models may be more accurate for the Nafion. Overall, the $I$ - $V$ characteristics of charge-selective channels and membranes appear to be primarily governed by electro-diffusion, so the modeling, specifically the membrane models, will neglect fluid flow.

The models used here treat the membranes as a uniform solid with a fixed surface charge and an effective diffusivity different than bulk (to be specified/fitted, etc.) Two types of 
model may be distinguished, the so-called ideally permselective model, which permits no coion current through the membrane and has a constant and uniform density of counter-ions with total charge equal and opposite that of the fixed charge. So-called non-ideal membranes permit ions of both sign, but most models (including those in Chapter 2) assume the membrane obeys local electroneutrality so that the sum of co-, counter- and fixed charges is zero everywhere inside. This assumption is investigated in Chapter 4.

\section{Permselectivity and double-layer overlap}

The two types of perm- (or equivalently, charge-) selective membranes, PC-NCM and Nafion, used in the experiments studied here both acquire their perm-selectivity because of overlapping EDL's inside the nanopores of the membranes. The basic picture of the mechanism is that the overlap of the electric double layers implies that the channel is mostly filled with positive ions which more-or-less compensate the fixed surface charge. However, since the layers overlap, they do not quite reach bulk electro-neutral conditions, i.e. the excess positive charge in solution balances the fixed charge on the walls and the net electrical potential inside the pore is negative, rising to zero infinitely far away in some bulk reservoir. In equilibrium, this implies that for the electro-chemical potentials of all species to be continuous in space, there will be a decreased number of co-ions (negative) inside the membrane. It is typically assumed that the structure of the double-layer deep inside the pore does not change dramatically (for an argument to this effect, see [54]) even under non-equilibrium conditions. Thus, double-layer overlap effectively limits transport of co-ions. It is also often assumed that the pore is more-or-less locally electro-neutral including the surface charge, i.e. that no charge-separated fronts develop over scales appreciably larger than the appropriate Debye length. This may not hold in strong non-equilibrium conditions and there is, to the author's knowledge, no conclusive research on the subject.

Assuming that the essential relationship between EDL overlap and permselectivity doesn't change much under non-equilibrium conditions, three ranges of overlap can be (very roughly) distinguished and result in three essentially different transport regimes: 
- strong overlap

- intermediate overlap

- weak to no overlap

Strong overlap results in very few co-ions passing through and has a nearly uniform distribution of potential throughout the (equilibrium) pore. Nanochannels/pores with strong overlap might be reasonably well modeled by ideal membranes. Weak overlap implies essentially bulk-like transport and is taken to refer to the case when the double-layer thickness is much more than about an order of magnitude or so smaller than the channel width, but this is by no means rigorous. The division between 'weakly selective' and 'non-selective' may be practically made by considering whether or not the channel exhibits responses consistent with charge-selective transport (such as concentration polarization in adjacent solution layers, to be discussed in the following section.) Intermediate permselectivity results when the double-layers are an appreciable fraction of the channel, and like the other definitions, is hardly rigorous. The non-ideal models with adjustable selectivity may prove the most useful. The effects of permselectivity on non-equilibrium transport are discussed next.

\subsection{Non-equilibrium electro-diffusion through charge- selective membranes}

This section provides an overview of concentration polarization, related effects, and important terminology. While fluid flow inside nanochannels, particularly the EOF generated by nanochannels themselves[27], may be important in obtaining good quantitative predictions for NCM's, it is clear from the previous discussion that the $I-V$ characteristics are primarily controlled by electro-diffusion. Therefore it is not essential to include fluid flow in the model to understand the basic $I-V$ response and ion-exchange membrane models should 
yield a qualitative understanding of related phenomena in the NCM and Nafion NMI devices. While NCMs have been reported to generate strong EOF, other authors have cited the high hydrodynamic resistance of single nanochannels as justification for the neglect of fluid flow through NMI systems $[8,66,64]$. However, electro-osmotic effects outside of membranes and nanochannels are known to play a critical role in the high-voltage characteristics of NMI systems $[50,51,68,64,66,5,67]$. This section also includes some brief discussion of related electro-osmotic effects and related open questions.

\subsubsection{Concentration polarization and (quasi-) steady-state membrane- electrolyte $I-V$ characteristics}

Consider a 3-layer 1D electrolytic cell such as shown in Figure 2.3. Upon application of the voltage, the positive ions (cations) migrate towards the cathode and the negative ions (anions) migrate towards the anode. For simplicity, consider the membrane to be ideally cation-selective, so that there are no anions present inside the membrane itself and the only negative charge is due to the fixed charge of the membrane itself. During an initial transient

period, excess anions will begin to accumulate on the right side of the membrane and deplete on the left side. For the regions beyond the Debye layer to maintain local electro-neutrality, the cation and anion fluxes must be equal. Therefore an excess of anions builds up on the right side of the membrane, slowing the migration of cations through the membrane and causing an overall increase in salt concentration. As the concentration gradients of both species increase, both species diffuse towards the cathode, e.g. there is a net flux of salt, and this layer thickens, growing towards the cathode until it fills the right side of the system.

On the left side of the membrane, the situation is slightly more complicated. The absence of anionic flux through the membrane causes the concentrations of both ions to deplete at the interface. Anions are drawn away from the membrane and at sufficiently low voltages corresponding to low currents, i.e. currents below the so-called classical limiting value, local electro-neutrality is maintained as the cation concentration is simultaneously lowered to a value dictated by the current density. At higher currents, the system cannot maintain local electro-neutrality in the depleted region. The two cases are considered separately below. 


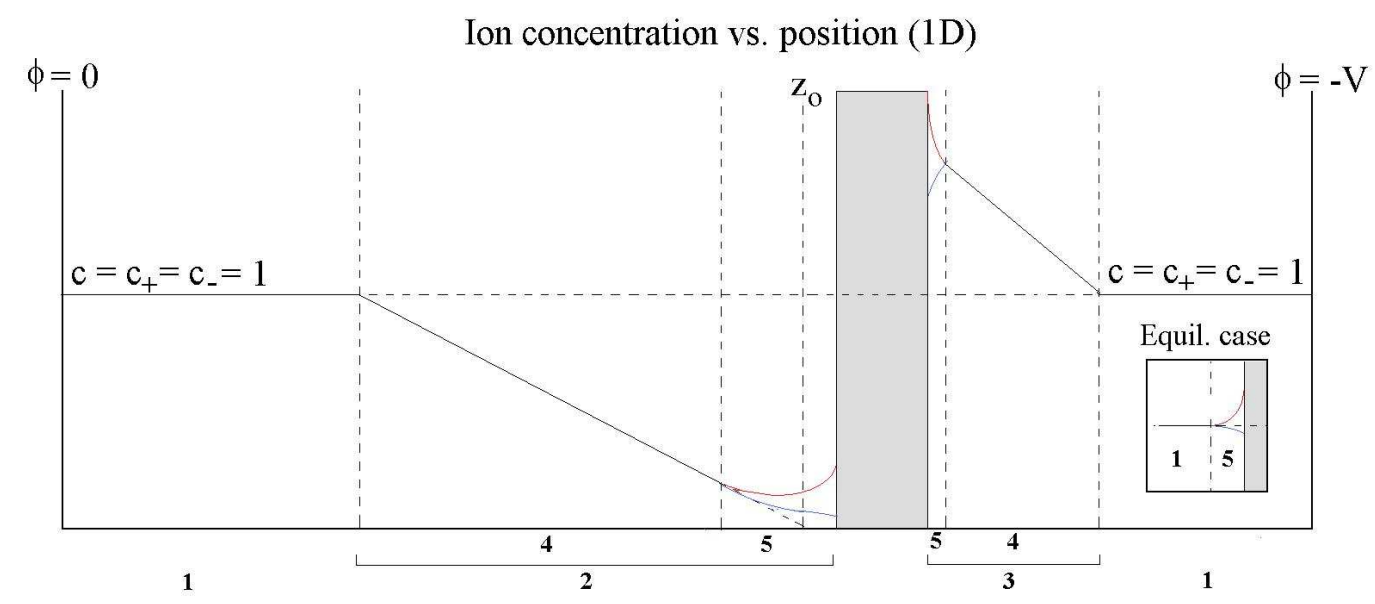

Figure 2.3: Anatomy of 1D concentration polarization layers (CPL) near a cation-selective membrane showing the LEN salt concentration in black and the cation (red) and anion (blue) concentrations. Five distinct regions are identified. Regions 1 denote electrolyte at bulk concentrations. This situation is somewhat artificial in the sense that $\mathrm{CP}$ will spread out from the electrodes towards the membrane as well, however if the electrodes are in large enough reservoirs located far from the membrane, the picture is realistic enough. Following the terminology of Ref. [68], regions 2 and 3 are the left and right quasi-1D diffusion layers (Q1DL) and regions 4 are known as the quasi-electroneutral bulk (QEB), which may be taken to be the same as the diffusion layers when space-charge can be ignored. Regions 5 correspond to the charged Debye layers and the depleted membrane-electrolyte interface can thicken above the classical limiting current. 


\section{Classical limiting current}

After a sufficient amount of time has passed, on the order of the diffusional time-scale $L^{2} / \mathcal{D}_{o}$ (where $L$ here is identically the linear size of the system) a steady-state is reached wherein the overall ion distributions are bi-linear functions of position and current, i.e. the stoichiometric salt concentration $c \equiv\left(c_{+}+c_{-}\right) / 2$ is $c \propto I x$. The true steady-state corresponds to the situation where the diffusion layers reach from the membrane surfaces to the electrodes. However, these layers change on a sufficiently slow scale in comparison to concentration changes across the membrane. After a few seconds (for a few microns-thick membrane) the current may be considered quasi-steady. Since the anion current is zero for the ideal case, it can be shown that it is given by the Boltzmann formula, $c_{-} \propto \exp \Phi / k T$ and that the electric potential is a logarithmic function of current density and position. Similar ion distributions arise in the more general case considered in Chapter 2, and details will be given there. For now, it is sufficient to note the qualitative behaviors. This picture of steady electric current and locally electro-neutral ion distributions is known as the classical picture of concentration polarization and is due primarily to Levich [28].

Since the salt concentration is linear in the current and position, there exists a maximum current corresponding to the concentration going to zero at the membrane surface. And, noting that the potential is logarithmic in the concentration, the interfacial value of the electric potential approaches a singularity as the current approaches the maximum current. The asymptotic approach to this maximum value at increasingly higher applied voltages corresponds to a decreasing interfacial conductivity, or equivalently an infinitely increasing differential resistance. This same limiting current phenomenon can be understood as the leading-order behavior for the more general case. 


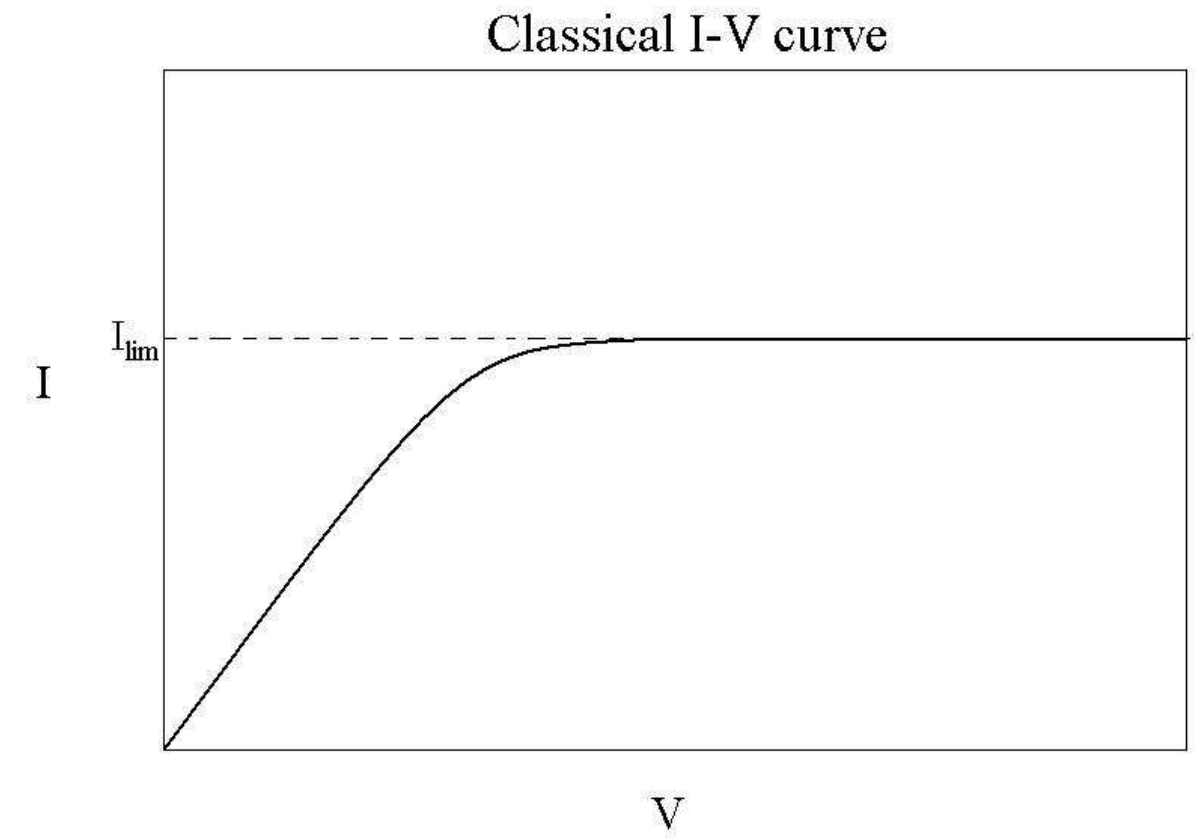

Figure 2.4: Typical form for a classical $I-V$ curve showing the diffusion-limited current saturation associated with the decrease of interfacial ion concentration. 


\section{LEN breakdown and over-limiting currents}

As the interfacial concentration approaches zero, the concentration within the membrane remains higher, at some (usually assumed) uniform and constant density equal to the density of fixed charges within the membrane. This implies an increasing cation concentration gradient, therefore cation diffusive flux, out of the membrane and into the depleted diffusion layer with increasing voltage. Since the salt concentration itself is approaching zero, the additional cation flux results in the build-up of an excess positive space charge in the depleted region adjacent to the membrane.

The development of extended space charge has been studied numerically, [47, 46, 35] and theoretically [49] and can be associated with a number of effects [12, 50, 68]. Some of the relevant details will be discussed later in this chapter. The motivation behind this body of work, particularly the initial numerical investigations, was the fact that experimentally obtained $I-V$ characteristics frequently do not exhibit the limiting current phenomenon, and when observed, it takes the form of a finite-width (in terms of voltage) plateau followed by an over-limiting current qualitatively similar to the curve in Figure 2.5.

The over-limiting conductance is generally attributed to mixing between the depleted region and bulk which occurs due to electro-convective vortices that develop in such systems at sufficiently high voltages. A number of studies [53, 64, 67] have supplied experimental evidence supporting the notion that the over-limiting conductance itself is due to mixing which is preceded or caused directly by the development of vortices which arise due to a non-equilibrium electro-osmotic slip occurring at the highly polarized extended space charge which develops above the limiting current $[50,51,68]$. There are other contributing factors as well, in particular so-called EOF of the second kind, which is vortex formation due to tangential electric field components acting directly on the space charge [12]. These vortices have been determined to diminish the width of the limiting current plateau, but are not believed to be directly responsible for the mixing and over-limiting current [66].

While it is certainly the case that suppressing electro-convection eliminates the overlimiting current [53] and that the over-limiting current can be correlated with a sequence of events which includes the development of an apparently stable vortex array displaying 


\section{Typical I-V curve for a system with permselective membrane}

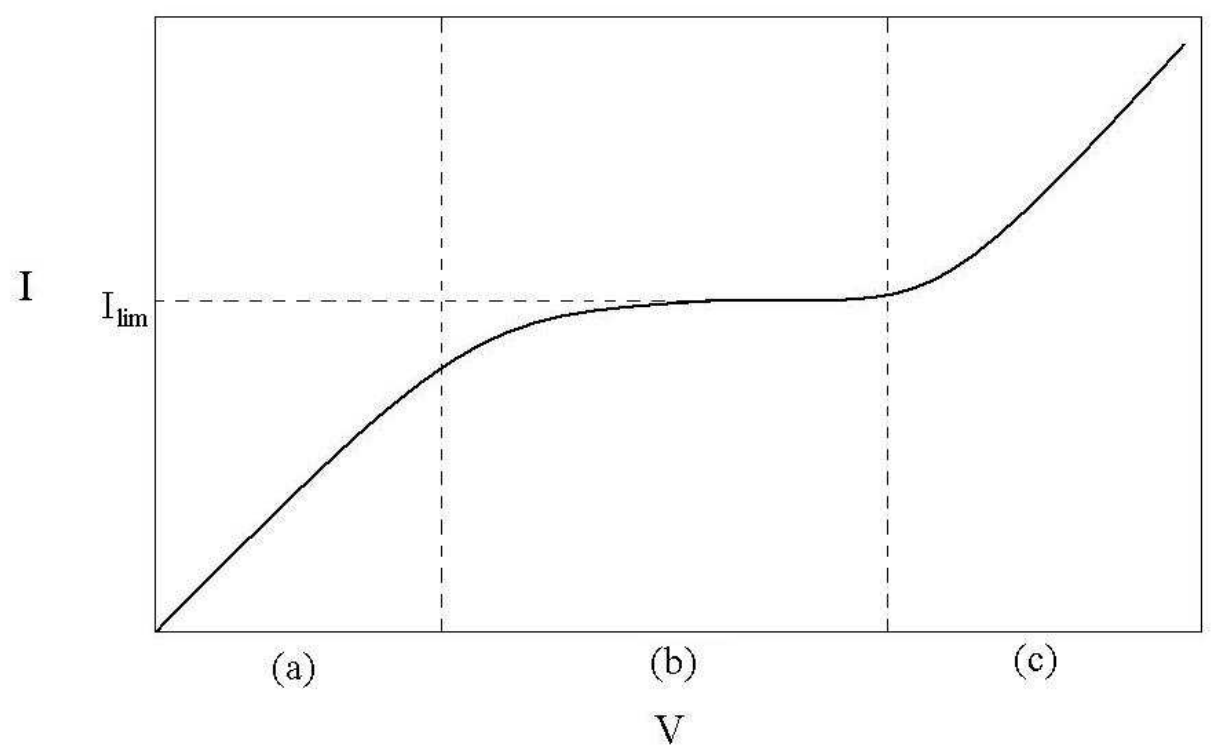

Figure 2.5: Typical features seen in an experimental $I-V$ curve for a membrane-electrolyte system with a sufficiently non-viscous fluid (i.e. water) as the solvent. (a) At low voltages, there is an Ohmic response region. (b) The limiting current begins to saturate near a critical voltage. Depending on the system, the limiting current region may have either a small or pronounced slope, but typically less than the slope in the Ohmic region. (c) Finally, at a second critical voltage, charge-selective systems typically develop a severe over-limiting response with a slope equal to or higher than the Ohmic slope. Note the over-limiting region may be suppressed by using a highly viscous fluid or gel instead of a relatively in-viscid aqueous electrolyte.[53] 
characteristics indicative of the vortices predicted by Rubinstein and Zaltzman's theories, certain questions remain un-answered. Perhaps most importantly, the effective depleted diffusion layer length - or more likely, the size of the space charge layer in region 5 of Figure 2.3 (equivalently, region C of Ref. [49])-is clearly selected by a process related to the formation of an electro-convective vortex array [64] but the observable size is not predicted by the theory. There is certainly no question that the vortices due to electro-convective instability of the non-equilibrium space charge region $[50,51,68]$ occur and coincide with the development of over-limiting current $[64,5,66,67]$.

What is not clear is whether or not the instability of the double-layer that allows the vortex-array to develop is responsible for arresting the growth of the depleted region nearest the electrode, thus selecting the vortex size, or if some other stability-related process limits the size of this region and/or the adjacent electro-neutral layers. In fact, the entire sub-issue of diffusion-layer dynamics is not well-understood (in terms of a strong consensus across the electrokinetics community. ${ }^{6}$ ) Some comments regarding various open questions and problems related to transport in systems with charge-selective elements are given in the following sub-sections.

\subsubsection{The problem with locally electro-neutral membranes and the existence of extended space charge}

Consider the typical model system used to investigate extended space charge and related phenomena such as used in Refs. $[49,50,51,68]$. In these cases, an electrolyte is located between two ideally cation-selective membranes wherein the cation density is considered fixed and equal to the membrane charge density, thus maintaining a locally electro-neutral membrane. Since the membranes are ideal, the number of anions between the two membranes must be held constant while it is usually assumed that additional cations may be drawn from reservoirs, which is a physically reasonable assumption.

\footnotetext{
${ }^{6}$ This statement could be updated somewhat; diffusion-layer dynamics is well understood in simple geometries, in 1D and 2D, but complex geometries and 3D problems are a topic of current research.
} 
Below the classical limiting value, there are no obvious problems with this model; ions re-distribute and the side of the system closest to the cathode becomes depleted while the anodic end becomes enriched with respect to the initial (bulk) values, maintaining local electro-neutrality everywhere except within the Debye layers. Above the limiting current, nothing much changes on the anode side except the concentration can reach even higher enriched values, as it is proportional to the current. However, the development of space charge at the cathode causes some problem. If the bulk-like reservoirs (not formally considered) and the membrane interiors are both to maintain local electro-neutrality, how is the development of extended space charge compatible with the requirement of charge conservation, i.e. of global electro-neutrality? It seems unreasonable to suppose the reservoirs (which are not explicitly included) develop significant space charge. A more questionable assumption, however, is whether or not the membrane maintains local electro-neutrality. This question could be addressed with a theory that both permits a non-negligible fraction of co-ions and does not a priori impose membrane electro-neutrality. This is effectively the same as asking the question "what is the source of the extended space charge?"[48]. This issue is taken up in Chapter 5.

\subsubsection{Transient effects, diffusion-layer dynamics, and a quasi-static approximation}

A large body of theory and modeling of microfluidic-nanofluidic devices and other electrokinetic systems employs a steady-state model [46, 62, 23, 22, 8, 64, 66, 47] to study the $I-V$ characteristics of electro-kinetic systems. In some cases, particularly systems which operate for long times compared to the LEN time-scale corresponding to the largest relevant length, this approach is fully justified. In other systems, specifically the NCM/NMI and the experiments of Chang, Yossifon, and collaborators (see particularly [64],) it is not clear that this is a valid approach.

Consider the model 1D system from Figure 2.3for conceptual ease. After a sufficiently long time, the transient (relaxational) anionic flux to the anode-region will die off completely 
and the transient component of the cationic flux to the cathode-region will likewise decrease until the cation flux reaches a steady-state value equal to the total electric current. A valid question is whether or not the total electric current itself changes under application of a fixed voltage. Experimentally (see Chapter 4) -in the absence of significant fluid-flow- it would seem that after some initial re-distribution of ions across the membrane and the formation of any charge-separated regions (less than or on the order of Debye lengths at low voltages) that it does not, despite the much slower (LEN) stoichiometric re-distribution of ions due to diffusion on either side of the membrane. However it should be emphasized that, at present, the topic of transient behavior and the various relevant time-scales in such systems is the subject of on-going research $[1,52]$.

The relevant time-scales themselves are partly determined by the magnitude of the current driven through the system. Above the limiting current, additional intermediate electrodiffusive time-scales can become important, not to mention electro-convective phenomena which will also have corresponding time-scales. Ignoring all of these complications, there is still the issue of the growth of the entire CPL. For a an electrodiffusive system to reach a true steady-state, the entire device length becomes concentration polarized, which requires a finite amount of time. For systems that have sufficiently long distances $(\sim 1 \mathrm{~cm})$ between electrodes, the system may not reach a true steady-state. So the operative question becomes whether or not, or under what conditions, a quasi-steady-state description might be employed. The evidence in Chapter 3, as well as several of the references seems to indicate this may be done much of the time (or at least is done in practice, [5].)

\section{Quasi-static electro-diffusion}

The following discussion outlines a suggestive argument for the validity of a quasi-static approach, but by no means offers rigorous proof. First, the quasi-static approach can be justified below the classical limiting current (in the absence of fluid flow) by appeal to experiments and scaling arguments. Preliminary simulations [3] and the experiments in Chapter 4 both seem to justify this claim empirically. The scaling argument is in the spirit 
of regular perturbations.

The following is asserted without any attempt at proof, but by appeal to analogy with the appearance of mixed time-scales above the classical limiting current $[1,52]$.

If a voltage is applied to the system, after a time longer than (a few multiples of) the characteristic diffusional relaxation across the membrane, i.e. $\delta^{2} / D_{o}$, a reasonable choice for the time-scale is

$$
t_{s}=\frac{\delta L}{D_{o}}
$$

where the membrane thickness is $\delta$ and the electrode distance is $L$. With this choice of time-scale, either $L$ or $\delta$ may be chosen as the length scale, since both imply quasi-static currents in leading order. The sub-limiting problem is given by the solution of an electroneutral diffusion equation, in $1 \mathrm{D}, c_{t}=\mathcal{D} c_{x x}$ [49]. The suggested scaling introduces a factor of $\delta / L$ on one or the other side of this equation. The left-side is multiplied by this factor if the length is scaled by $\delta$ and the right side if the length is scaled by $L$. In either case, the leading-order problem is time-independent. The distinction is one of point-of-view. Choosing the length scale as the membrane thickness implies that interest is focused on the structure of the diffusion layers when they are on the order of or larger than the membrane thickness, yet considerably smaller than the device length. Conversely, choosing the device length implies that interest is focused on the concentrations far from the membrane, where they are approximately both uniform and steady-state for times less than $L^{2} / D_{o}$, which can be on the order of hours (see Ch. 3.) Accordingly, the application of a quasi-static approach can be expected to yield the best results for systems with thin membranes in comparison to the electrode distance at sub-limiting currents for measurements of reasonable duration in which fluid flow is negligible.

\section{Transient diffusion layer growth}

Above the limiting current, as mentioned previously, the CPL undergoes a fundamental structural change [49]. The Debye layers begin to thicken as the concentration at the membrane surface drops to zero. The growth dynamics of the problem reflect this change, and 
while the growth of the electro-neutral portion of the CPL structure, the so-called quasielectroneutral bulk, retains the usual diffusive character, the space charge growth is driven by dynamics that resemble front formation, i.e. a growing free boundary [49].

The electro-neutral diffusive growth problem is well-understood. An equivalent version of the sub-limiting problem has been analyzed by Hunter [18] and this result has been compared to experiment [64]. Some of the more important points of the analysis and experiments will be discussed in this section. The analysis of the problem in Ref. [49] is somewhat formal and does not deal with explicit spatio-temporal profiles. However, practically speaking, the calculation of explicit time-dependent profiles is of limited use for fluidic systems since a length selection process related to the formation of electro-convective vortices is known to become operative above a critical voltage corresponding to over-limiting currents [64]. A comprehensive and predictive understanding of this length-selection process is one of the major open questions in the field.

In both sub- and super-limiting conditions, the electro-neutral portion of the CPL maintains diffusive growth dynamics. Hunter solves a 2-component electro-diffusion problem on the half-plane with a bounding surface emitting one species of ion at a flux density $j$ per unit area [18]. His analytic result keeps first-order terms, linearizing about the equilibrium solution by an arbitrary perturbation of the salt concentration and charge. Incidentally, this is essentially the same as the quasi-static approach above and suggests that since changes in the concentration from equilibrium $c$ are first-order, any time-dependence in the current would be first-order in $\delta / L$ as well.

The salt concentration perturbation is then

$$
c(x, t)=\frac{j}{2 \sqrt{\mathcal{D}}}\left\{2\left[\frac{t}{\pi}\right]^{1 / 2} \exp \left(-x^{2} / 4 \mathcal{D} t\right)-\frac{x}{\sqrt{\mathcal{D}}}\left[1-\operatorname{erf}\left(\frac{x}{2 \sqrt{\mathcal{D} t}}\right)\right]\right\}
$$

This expression may be used on either side of the membrane to describe the change in salt concentration, with decrease or increase denoted by the sign of the flux density $j$ for the selected ion. Hunter then gives a simplified expression, valid for large distances as

$$
c(x, t) \cong \frac{2 j t^{3 / 2} \sqrt{\mathcal{D} t}}{x^{2}} \exp \left(\frac{x^{2}}{4 \mathcal{D} t}\right) .
$$


This expression holds sufficiently far from the membrane, i.e. for distances much greater than $\sqrt{\mathcal{D} t}$ and the width of the advancing concentration disturbance may be estimated by $\sqrt{\mathcal{D} t}$ Yossifon et. al. [64] have shown that this estimate for diffusion layer thickness holds reasonably well up to the onset of vortex-array formation and over-limiting conductance. This confirms the basic picture of diffusive growth in a realistic system. However, it also seems to indicate that below the onset of vortex-formation, the presence of the free-boundary dynamics associated with the growing space charge do not radically affect the observable response of nanochannel systems.

As will be considered in more detail in Chapter 5, one other important observation that can be made from the Yossifon et. al. experiments as well as the NCM/NMI experiments is that the apparent onset of vortex-driven overlimiting conductance corresponds well with Zaltzman and Rubinstein's estimates of the loss of stability of the so-called Q1DL [68], i.e. the point at which tangential components cannot be neglected in any problem. However, the relationship between the vortex dynamics, depleted Q1DL space charge region length selection, and QEB diffusive growth is not completely understood. 


\section{Chapter 3}

\section{A classical electro-diffusive model of fluidic rectification}

\subsection{Chapter introduction and overview}

The main goal of this chapter is to study fluidic rectification in the simplest context possible and demonstrate how it is fundamentally a result of underlying asymmetry in the micro-nanofluidic device. In the course of doing this, several variations on a simple model are considered which yield some qualitative and semi-quantitative predictions for the behavior of electro-diffusive rectifiers.

Section 3.2 gives a brief review of the wide variety of devices and models that have appeared in the literature over the years along with a classification scheme for the different kinds of asymmetry that result in fluidic rectification. This allows fluidic rectification to be understood in general terms, i.e. how the device asymmetry - due to asymmetric surface properties, geometry, or both-induces an asymmetry in the flux densities of the ionic species, causing asymmetric concentration polarization, which in turn results in a net current that is asymmetric with respect to the direction of the applied electric field. Section 3.3 discusses possible mechanisms by which geometric asymmetry in the microfluidic portion of the system results in asymmetric concentration polarization.

A 1D model using the locally electro-neutral approximation to the full Poisson-Nernst- 
Jarrod E. Schiffbauer Chapter 3. A classical electro-diffusive model of fluidic rectification 44

Planck formulation of electro-diffusion is developed in section 3.4. The asymmetry is represented here by diffusion layers of different lengths. Both ideally perm-selective membranes, which completely restrict the passage of co-ions, and non-ideal membranes which permit both co- and counter-ions to pass through the membrane, are examined in detail. This specific model permits the calculation of model $I$ - $V$ curves, concentration and potential profiles, selectivity versus voltage, and rectification factors. These are used to study the effects of various representative parameters such as overall asymmetry, membrane charge and diffusivity in various limits. While this simplified model cannot be anticipated to capture all of the details present in a realistic 3D system with charged nanochannels, it does contain the essential qualitative relationships and provides an intuitive understanding of fluidic rectification.

Section 3.5 demonstrates how asymmetric diffusion layer (DL) lengths compare to a more general asymmetry in the concentration polarization layer (CPL) structure due to a fluxfocusing effect. This is accomplished via a basic extension of the 1D model to a quasi-1D approximation of a 2D model wherein the flux density is considered to vary discontinuously, but the net flux is conserved. The effects are essentially similar to those in the genuine 1D model with different diffusion lengths.

Finally, section 3.6 recapitulates the main results and discusses on-going work as well as some open questions.

\subsection{A review of fluidic rectification}

\subsubsection{Historical development, device types}

\section{Earliest work}

The underlying ideas for fluidic rectification have been developed over the last 50 years. Two independent observations of the similarity between doped semiconductors and the de-

ficiency or excess of protons in aqueous alkaline or acidic solutions $[45,13]$ motivated some simple experiments with bi-polar ion exchange membranes in which fluidic rectification was 
Jarrod E. Schiffbauer Chapter 3. A classical electro-diffusive model of fluidic rectification 45 observed [31]. Over the intervening years, membrane-based systems with similarities to biological membranes were shown to exhibit similar effects [20]. About 40 years after the first membrane rectifier, Wei et al. observed fluidic rectification in quartz nanopipette electrodes [62]. Wei et al.'s work was concerned primarily with the improvement of spatial resolution in a relatively new technique known as scanning electro-chemical microscopy, and not LOC/ $\mu$ TAS systems.

The pipette-electrodes have a conical aperture on the order of nanometers across at the entrance, and the silanol-like surface groups on quartz develop a surface charge according to a mechanism similar to the site-binding/dissociation mechanism in Ch. 2. However, Wei et al's models did not explicitly treat the modulated transport properties inside the membrane, assuming instead an Ohmic current-voltage relationship for the counter-ions in the nanochannel itself with an adjustable perm-selectivity parameter and treating the adjacent solution as being confined to infinitely long cones of differing solid angle. Wei et al. were able to obtain I-V curves that corresponded qualitatively to experiments, showing a rectification response similar to that observed in membrane-based systems. However, given the two very different systems, it is reasonable to wonder how the mechanism responsible for rectification in Wei et al's pipettes compares to that in Lovrecek et al's bi-polar membranes. Wei's work attributed their observations to two phenomena: charge-selective transport (due to double-layer overlap in the nano-portion of the pipette) and asymmetric entrances to the nano-sized region. Lovrecek et. al offered no theoretical explanation, however it is clear that the device exhibits asymmetry at the junction between the two perm-selective membranes.

Following the work of the Wei (Bard) group, interest and development in fluidic rectification stagnated for a few years until the development of microfluidic devices with nanofluidic interconnects gained interest in the research community. Around the same time, two different classes of nanofluidic-based rectifiers were being studied, primarily by two different groups. Phenomenologically, these two classes of device appear to be representative of the two types of earlier fluidic rectifier. 
Jarrod E. Schiffbauer Chapter 3. A classical electro-diffusive model of fluidic rectification 46

\section{Geometrically symmetric nano-fluidic diodes}

The work of the Daiguji group and collaborators was concerned with fluidic rectification in symmetric nanochannels $[8,22]$ and gating/control via electro-static addressing of surface properties in such devices $[9,8,23]$. These devices did not exhibit the kind of geometric asymmetry believed responsible for the rectification in the Wei pipettes, but instead contained a junction in the nano-channel between regions with different surface charge. Theoretical studies by the Daiguji group considered both scenarios in which half of the channel surface is positively charged, and the other half negatively charged. Thus each side would preferentially carry ions of opposite sign, making these devices the nanofluidic analogues of the early bi-polar membranes. They, as well as Vlassiouk [60], also considered uni-polar diodes - cylindrical diodes wherein only the magnitude of the charge was modulated. Characterization of the experimental set-up [22] showed that the uni-polar scenario more likely provided the correct description of the operation of their devices with differential surface coating.

For the gating-electrostatic control experiments, field-effect-like control of nano-fluidic transport is achieved by applying a voltage to gate electrodes deposited across the middle of the nano-channels [23] which modulate the double-layer. Some modeling work, mostly numerical, by these researchers, led to similar conclusions as the earlier work by Wei, et. al regarding the necessity of charge-selective transport due to double-layer overlap and the role of depletion zones in the occurrence of a reversed-bias or off-state for these devices. However, the focus of these studies was on the role of the effects of modified surface properties rather than the fundamental rectification mechanism itself.

\section{Siwy group's conical nanopore papers}

The other development, occurring roughly parallel to the work of the Daiguji group, concerns work done on conical nanopores by Siwy and collaborators [55]. Much of this work focused on the transport properties of small conical pores with a range of tip openings from a few to tens of nanometers and base openings of a few hundred nanometers. In addition 
Jarrod E. Schiffbauer Chapter 3. A classical electro-diffusive model of fluidic rectification 47 to rectification effects, Siwy and co-workers also demonstrated asymmetric transport due to an applied concentration gradient [56] and gating-control effects [21] similar to those in the symmetric nano-transistors of Daiguji and Karnik.

The asymmetric transport induced by the concentration gradient is essentially the complement of the rectification effect and is suggestive of a cross-coupling between transport response functions, i.e. how, if at all, does the asymmetric system alter form of the Onsager relations for steady-state electrokinetic transport in symmetric systems[36, 4]? Similar effects are also present in the so-called chemical ratchet application of the NCM/NMI (see Ch. 3.). The Siwy group has done some theoretical work in addition to the experiments (see [55] and references therein), but of a significantly different character (at first glance) than the electrokinetic models introduced in the previous chapter. The relationship between the types of model invoked by the Siwy group to explain rectification in conical nanopores and other models will be explored somewhat later on in this overview. But a comprehensive comparison- like a detailed consideration of the Onsager relations- is left for future investigation.

\section{Thimble geometry of Jung et. al.}

Jung and collaborators have reported observation of the rectification effect in a thimblelike geometry consisting of a single $175 \mathrm{~nm}$ diameter nanopore situated in the center of a $2 \mu \mathrm{m}$ diameter counter-sink at the end of a $100 \mu \mathrm{m}$ diameter microchannel [19]. A diagram appears in Figure 3.1 along with diagrams of several other rectifiers. Their results confirm the earlier results regarding the role of double-layer overlap and bulk-concentration on rectification and help to clarify an important point; the asymmetry need not be confined - or even exist at- the nano-meter scale in order to achieve fluidic rectification. This, like the NCM/NMI, represents a device with symmetric, (essentially) uniform surface charge inside of a symmetric, uniform nano-porous region in which the asymmetry resides entirely in the fluidic portion of the system. In both Siwy's conical pores and Wei's pipettes, it is not clear from actual dimensions and geometry in the experimental set-up that the geometric asymmetry outside the pore 
Jarrod E. Schiffbauer Chapter 3. A classical electro-diffusive model of fluidic rectification 48 plays an important role. However, Wei's model does suggest this, as will be discussed shortly.

\section{Rectifying nano-slot array of Yossifon and Chang}

Yossifon, Chang and collaborators have used single nano-slot and nano-slot arrays to experimentally investigate a number of important fundamental electrokinetic effects $[64,66$, $5,67]$ including fluidic rectification due to geometric asymmetry $[5,65]$. In these devices, the asymmetry arises because on one side of the device, the individual nano-slots in the array, each approximately 100 microns wide, enter directly into a reservoir and on the other side, they first join into a common wide nano-slot before entering the reservoir. This effectively increases the width of each nano-slot from 100 microns to 350 microns across the entrance region. For a diagram of an individual cell from such an array, see Figure 3.1 (b).

While it is tempting to associate this 350 micron-wide entrance as analogous to the base of a conical nano-pore, this is not the case. The device should rectify with or without this wide nano-slot entrance, though it may change the characteristics. The critical feature is that the narrower 100 micron ends have a non-negligible region in which there is no doublelayer over-lap - hence the region is not perm-selective- and mixing with the bulk is limited by the relatively narrow walls. The method of assembly involving an intentional mis-alignment of the top and bottom slides which make up the device creates the wide entrance nano-slot and ensures that the individual ends of the channels (at the right in Figure 3.1 (b)) behave essentially as micro-channels.

The design of Yossifon and Chang's devices permit them to use fluorescent dye at low (10 $\mu \mathrm{M})$ concentrations to image the growth of enrichment and depletion zones in the rectifier. The device exhibits CP effects consistent with those expected for the NCM and other rectifiers. In the on-state (forward bias) a depletion zone forms at the positively-biased (wide slot) side of the device and enriched zones are visible at the narrow cathodic ends of the 100 micron wide slots. The opposite is true when the bias is reversed.

Yossifon and Chang use a variation on the PNP-based model of Manzanares, et. al. [35] to study their systems. Some of the details will be discussed subsequently in comparison 
Jarrod E. Schiffbauer Chapter 3. A classical electro-diffusive model of fluidic rectification 49 to several other approaches to understanding fluidic rectification. The explanation for fluidic rectification they ultimately put forth is that it is due to asymmetric polarization in the extended space charge layers. This is justified based on the absence of significant rectification below (approximately) the voltage at which space charge effects are expected to become important and a very strong rectification factor at high voltages. While the model does support the notion that polarization of the extended space charge may significantly affect rectification, the results presented here suggest that the rectification effect is strongly influenced by classical concentration polarization.

\subsubsection{Comparison to the NCM/NMI: Flux- and field-focusing ge- ometries}

The NCM/NMI and Nafion/NMI I-V devices exhibit current rectification up to very high voltage. The NCM/NMI, as mentioned previously, contains an array of thousands of symmetric, cylindrical pores of $10 \mathrm{~nm}$ diameter connecting a micro-channel on one side with a larger cylindrical opening $(r \approx 0.8 \mathrm{~mm})$ which is connected directly to a macroscopic reservoir. The on-state (forward bias) occurs when the electric field and salt flux are directed from the large reservoir-side of the membrane into the micro-channel. In this case, an enriched diffusion layer forms in the micro-channel itself and depleted regions are expected to form in the reservoir opening. Under reverse bias, a depleted region has been imaged in the micro-channel (see Ch. 3 and Ref. [24]) and an enriched region is expected to form in the reservoir opening. While images of the micro-channel have been obtained and analyzed, because of the device design, it is not possible to image the reservoir opening. However, from an overall comparison with the other devices- including the V-channel NMI device in Figure 1.2 of Chapter 0 - it seems reasonable to assume the device behaves similarly to all of the other geometrically asymmetric devices above.

Upon consideration of the relative magnitudes and directions of the net salt flux density $J_{+}$, individual species flux densities, and the electric field direction for the geometrically asymmetric devices, it becomes apparent that all of them exhibit essentially the same be- 
Jarrod E. Schiffbauer Chapter 3. A classical electro-diffusive model of fluidic rectification 50 havior. It seems then likely that the behavior is due to essentially the same mechanism. In the on-state, as pictured in Figure 3.1, the wide regions to the left of the perm-selective region (shaded grey) will have a lower flux density at a given steady-state current than the narrow regions to the right of the perm-selective region. Because of conservation of net flux the diffusion layers forming in regions of low flux density will differ less dramatically from bulk-like conditions than those forming in the narrow regions of high flux density. This may be thought of as flux-focusing, in analogy to the field-focusing effect [66] acting at an NMI. At least for the cation-selective, geometrically asymmetric devices, the on-state occurs when the net salt flux and the cation flux are both being focused from a region of large geometric size, e.g. low flux density, into one of a smaller size/higher flux density. The relationship between geometry and diffusion layer growth will be considered in more detail in section 3.3. In section 3.2.3 below, the various theoretical approaches to understanding the rectification phenomenon are discussed further along with some general observations.

\subsubsection{Comparison of theoretical explanations, models, and exper- iments}

The majority of the work on fluidic rectification has included a modeling component, and in general these all yield $I-V$ characteristic curves with varying degrees of qualitative similarity to those observed experimentally. The models make a variety of assumptions, however all of them have two things in common: some kind of underlying asymmetry and charge-selective transport through regions with nanometer-sized passages.

The position put forth in this manuscript is that all geometrically asymmetric rectifiers rely on variations of a common mechanism. An examination of the body of published literature appears to support this position. The most essential and basic features of this mechanism can be understood using the model by Wei et al.. The flux densities on either side of a perm-selective region differ because of the geometric asymmetry - in Wei et. al.'a 
Jarrod E. Schiffbauer Chapter 3. A classical electro-diffusive model of fluidic rectification 51

(a)

(b)

(c)

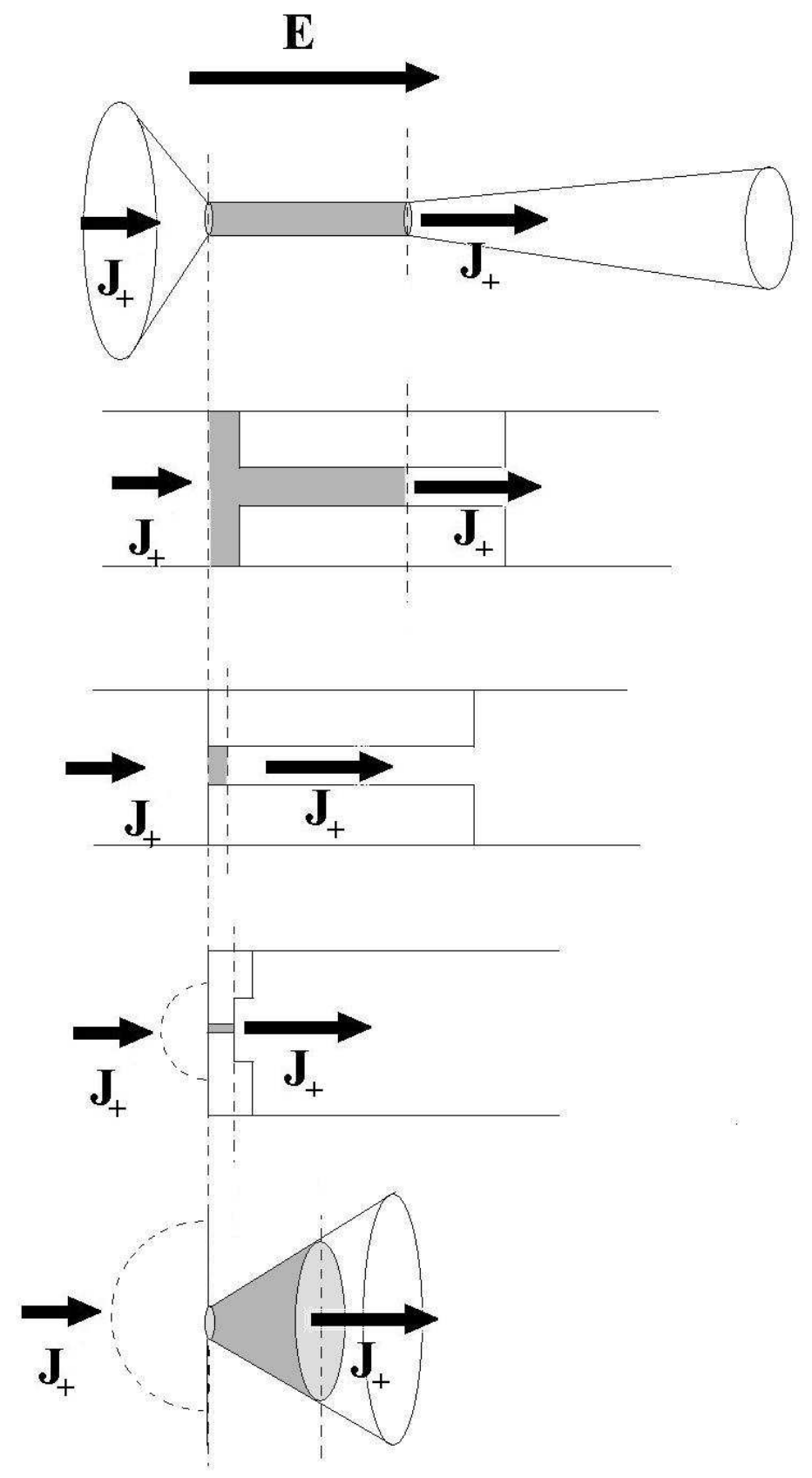

Figure 3.1: Flux-focusing geometries for several theoretical and experimental studies of fluidic rectification. (a) Wei's infinite-cone model, (b) A single channel 'cell' for the nanochannel array of Yossifon and Chang, (c) the NCM/NMI, (d) Jung et. al's thimble, and (e) Siwy et. al's conical pores. 
Jarrod E. Schiffbauer Chapter 3. A classical electro-diffusive model of fluidic rectification 52

case, a difference in solid angle- and this results in a difference in the net current through the device. Wei et. al. do not consider the details of the ion distributions in these infinitely long conical diffusion regions, so the connection between concentration polarization and fluidic rectification was not clear. However, this is evident in the $2 \mathrm{D}$ numerical solution for the steady-state PNP model of a conical nano-pore by Liu et. al. [30], where they correctly attribute rectification to the formation of asymmetric concentration polarization layers both inside and outside of the tip of the cone consistent with the diagram in Figure 3.1 (e.)

Liu et. al. make a number of important connections to other devices, comparing the conical nano-pore to a geometrically distorted diode, although they do not go into great detail. The comparison is most apt for the uni-polar diode, variations of which were studied by both the Daiguji group [8, 22] and Vlassiouk, et. al. [60]. A key point, noted by Vlassiouk, is that the rectification mechanisms for bi-polar and uni-polar diodes differ, with the bi-polar diode functioning somewhat analogously to a P-N junction and the uni-polar diode reminiscent of a Schottky diode, where the formation of a depleted region at a metal-semiconductor junction is responsible for the off-state response. In terms of the flux densities, the bi-polar diode rectifies because discontinuity in the individual species fluxes cause a depletion in the off-state and enrichment in the on-state. The effect does not require any field or flux-focusing and is asymmetric about the junction between cation and anion selective devices. The uni-polar diode rectifies because, like the geometrically asymmetric devices, a depletion zone forms inside the device in the off-state. While neither Vlassiouk nor Karnik's models for uni-polar diodes predict a depletion (in the on-state) or enrichment (off-state) outside of the diode body, if Liu's comparison between conical nano-pores and diodes is accurate, this should be expected to occur in a model which takes the CPL outside the nano-pore into account. In any physical device, field and flux focusing at the nano-pore edges would augment the internal effects predicted by these models.

The models employed by Siwy et. al. [55] to describe the potential inside a pore point to a more subtle relationship between geometrically asymmetric rectifiers and the space-charge modulated devices. These models result in ratchet-like potentials and are similar in many respects to transport models derived from a Fick-Jacobs type approximation to the problem of diffusion plus drift in geometries with constrictions or bulges $[69,44]$. In these latter stud- 
Jarrod E. Schiffbauer Chapter 3. A classical electro-diffusive model of fluidic rectification 53 ies, an effective 1D potential is derived for transport through regions where the cross-section and potential energy change along the axis, thus effectively introducing entropy and energy barriers. Accordingly, one may think of rectification in geometrically asymmetric devices as being an entropic effect and the cylindrical diodes as an energetic effect. If the area changes in a region where the sign or magnitude of the charge is also varying, there are both kinds of barrier. In [44] the situation is clarified further; purely energetic barriers correspond to (single-particle) probability distribution functions governed by a Fokker-Planck equation while purely entropic barriers are governed by a Fick-Jacobs equation. While such a kinetic description may seem most appropriate for very small (a few nm) nano-channels [40], in this case there is a clear physical and mathematical correspondence between a probability density and a number density. The connection between the continuum diffusion-advection equation and stochastic equations was even the subject of a Physics Today article [38]. The possibility of using this approach to model fluidic rectification is considered as a possible topic for future investigation.

The spectrum of experiments and models, taken together, demonstrates that fluidic rectification can arise due to electrical asymmetry, geometric asymmetry, or the combined effects of the two. In general, the asymmetry creates either an enriched or depleted region, the non-linear conductivity of which dominates the response of the entire system and is responsible, respectively, for the high-(average) conductivity on (forward-bias) or low-conductivity off (reverse) states. The principle differences between the devices are ultimately due to the manner in which the asymmetry affects the nature, location, and shape of these enriched and depleted regions.

The collective understanding of the mechanisms responsible for fluidic rectification may be summarized as follows:

- Flux densities inherit geometric and/or electrical asymmetries present in a system

- These cause the CPL to be asymmetric w.r.t. the direction of applied fields. This asymmetry may also be carried over into the non-LEN regions, causing asymmetric polarization of the double-layers and, at high enough voltages, the extended space 


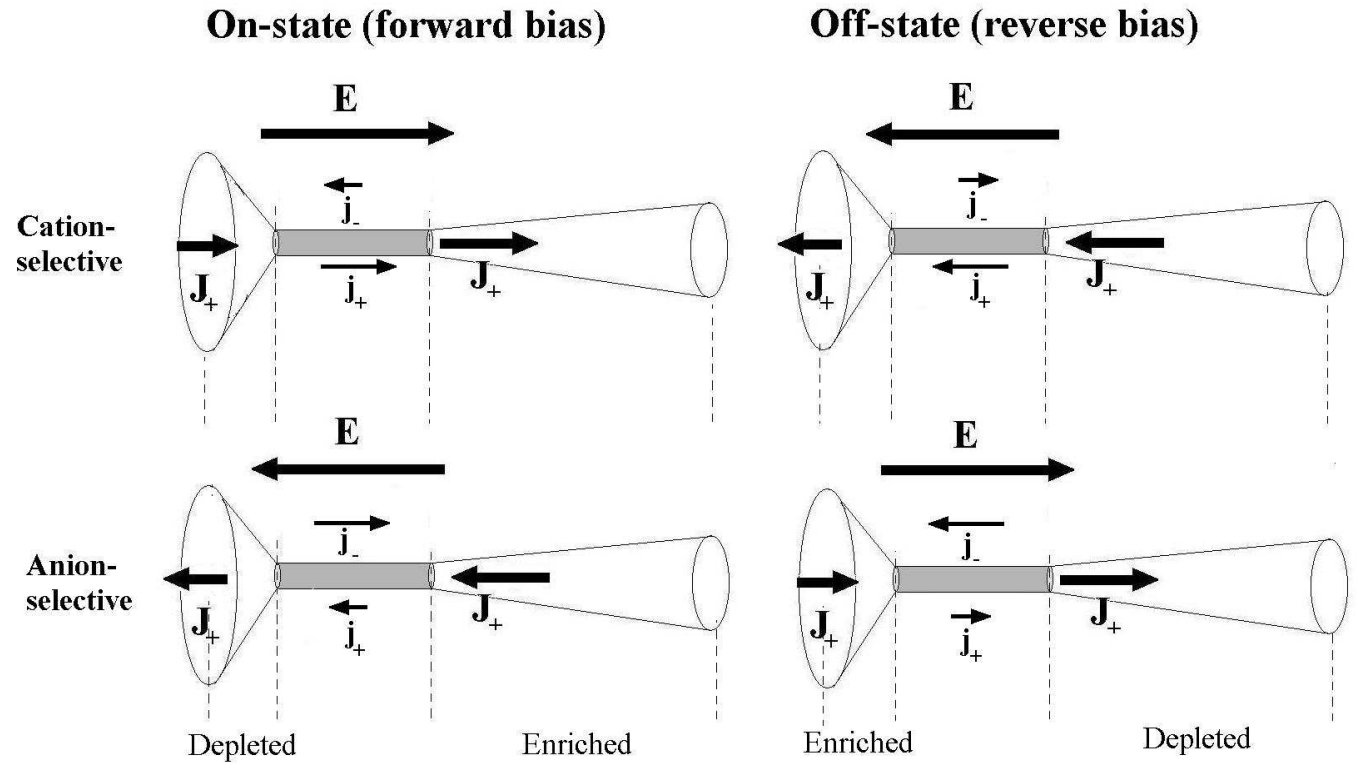

Figure 3.2: On and off cartoons using Wei's geometry and Pu's intuitive flux balancing model

charge.

- Regardless of the details of charge-selective conduction inside regions with overlapping double-layers, the asymmetric, non-linear conductivity/resistivity due to the CPL asymmetry -including polarization of the EDL/SCL- is such that the net conductivity is lower in one state than the other.

Using Wei et. al.'s model geometry and reasoning regarding the CPL formation similar to that in [43], the general picture appearing in Figure 3.2 emerges. Notice that, in terms of the charge-selectivity, the flux density of the majority carrier is converging in the on-state configuration and diverging in the off-state.

It would be desirable to clarify several things further using an intuitive analytic approach similar to that of Wei et. al. Since it is known that the magnitude of the current and selectivity can be influenced by conditions in the adjoining microfluidic/reservoir layers [59], it would be interesting to consider these effects as well. The next two sections of this chapter 
Jarrod E. Schiffbauer Chapter 3. A classical electro-diffusive model of fluidic rectification 55 employ simple arguments and results (see Ch. 2.4.3) and (quasi-) 1D steady-state models to address the following three questions:

- In general, how can fluidic geometry influence the CPL in geometrically asymmetric systems?

- What can be determined about the relationship between the CPL, properties of the nano-channel/membrane, and rectification response?

- How do these depleted and enriched regions influence transport inside the nano-channel/membrane?

These models are then used to make some qualitative predictions regarding the relationship between geometry, buffer concentration, and rectification effects for the NCM/NMI. In Chapter 3, experimental $I-V$ data from several NCM/NMI devices are analyzed and compared to these predictions and the model used to understand some apparent history effects in the system.

\subsection{The relationship between geometric asymmetry and asymmetric concentration polarization in the NCM/NMI device}

In the majority of the published studies where the effects of diffusion layers are explicitly taken into account (see references in previous section), is often assumed that the diffusion layers extend from the perm-selective interface to the electrodes and undergo no significant changes during the measurement. Provided the amount of time elapsed between the application of voltage and the actual measurement is sufficiently long (in comparison to the relevant device dimensions and species diffusivities, see Table 3.1) this is a safe assumption. However, since all disturbances propagate at a finite speed, it seems reasonable to suppose there are circumstances where this assumption fails to hold. Two theoretical results concerning the growth of diffusion layers adjacent to planar ion sources have been published. The first 
Jarrod E. Schiffbauer Chapter 3. A classical electro-diffusive model of fluidic rectification 56

Table 3.1: Estimates of characteristic time for diffusion of various ions present in the NCM/NMI experiments for $1 \mathrm{~cm}$ and 100 micron lengths. According to the universal scaling limit from Hunter's analysis [?], which is expected to be approximately valid for systems operating below the classical limiting current, this gives an estimate of the time required for diffusion layer growth across such distances.

\begin{tabular}{cccc}
\hline Ionic species & Diffusivity $\left(\mathrm{cm}^{2} / \mathrm{s}\right)$ & $\tau_{D}(\mathrm{~s})$ for $L=1 \mathrm{~cm}$ & $\tau_{D}(\mathrm{~s})$ for $L=100 \mu m$ \\
\hline $\mathrm{Na}^{+}$ & $1.32 \times 10^{-5}$ & 75757.6 & 7.57576 \\
$\mathrm{H}^{+}$ & $9.2 \times 10^{-5}$ & 10869.6 & 1.08696 \\
$\mathrm{OH}^{-}$ & $5.29 \times 10^{-5}$ & 18903.6 & 1.89036 \\
Fluorescein $(z=-2)$ & $1.70 \times 10^{-6}$ & 588235. & 58.8235 \\
\hline
\end{tabular}

result, see Ref. [?] can be shown to yield the standard diffusive scaling estimate, $\Delta x=\sqrt{\mathcal{D} t}$ for the width of a diffusion layer as a function of time.

A separate result, see Ref. [49] shows that the typical diffusive growth, i.e. the previous result in Ref. [?], is expected to hold for currents below the classical limiting current, but that above this current, a free-boundary problem of a different nature governs the growth of boundary layers. To the author's knowledge, this second result has not been analyzed in the limit of large distances and long times. The approximate formula for simple diffusive growth has been experimentally verified [64] to hold reasonably well up to the point where electro-convective instabilities occur $[68,50]$. A process related to the vortex-arrays, possibly the stability of these arrays, then selects the diffusion length according to an unknown mechanism.

The goal in the remainder of this section will be to obtain a rough idea of how the asymmetric cross-sections can influence the growth of the diffusion layers using the diffusive growth solution from [18]. The fact that the diffusive growth dynamics have been empirically shown to provide reasonably good quantitative agreement with the simple scaling law lends this approach some credence. However, the description here neglects many important effects and is intended to serve as means of gaining some initial understanding of the relationship 
Jarrod E. Schiffbauer Chapter 3. A classical electro-diffusive model of fluidic rectification 57 between fluidic geometry and diffusion layer properties.

\subsubsection{Flux conservation in a geometrically asymmetric discontin- uous system}

In the pseudo-1D model of the asymmetric NCM system, we consider two half-plane regions on opposite sides of the membrane with a flux rate of ions $J_{+, r}$ out of the reservoir and into the membrane and a flux rate $J_{+, \mu}$ into the microchannel from the membrane. Since the cross-sections of both differ, continuity demands that

$$
J_{+, \mu} A_{\mu}=J_{+, r} A_{r}
$$

where $A_{\mu, r}$ refers to the microchannel or reservoir, respectively. In a situation such as that depicted in Figure 3.3, this expression could be expected to be accurate far from the membrane region. Close to the membrane, since the active area of the membrane itself is the same as the channel (in this particular model,) the flux densities should be approximately the same on each side of the membrane and a realistic calculation would be expected to show a region of nearly hemispherical growth which would persist until the diffusion layers reached the channel walls, at which point the growth might be expected to more closely resemble the quasi-1D picture presented here.

It is conceivable that other geometries, particularly ones similar to the devices of Yossifon et. al. [65] would exhibit front growth which is much closer to the quasi-1D front growth. Nonetheless, the model here gives some useful ideas regarding diffusion layer growth. Moreover, the point is that the difference in cross-sectional area is anticipated to have two different effects:

- A flux-focusing effect, analogous to the field-focusing effect [66]

- An effect upon the relative lengths of (quasi) 1D diffusion layers

The effects of flux-focusing are considered in section 3.5. In this section, the possible effects of discontinuous cross-sectional area on the DL growth are considered. 
Jarrod E. Schiffbauer Chapter 3. A classical electro-diffusive model of fluidic rectification 58

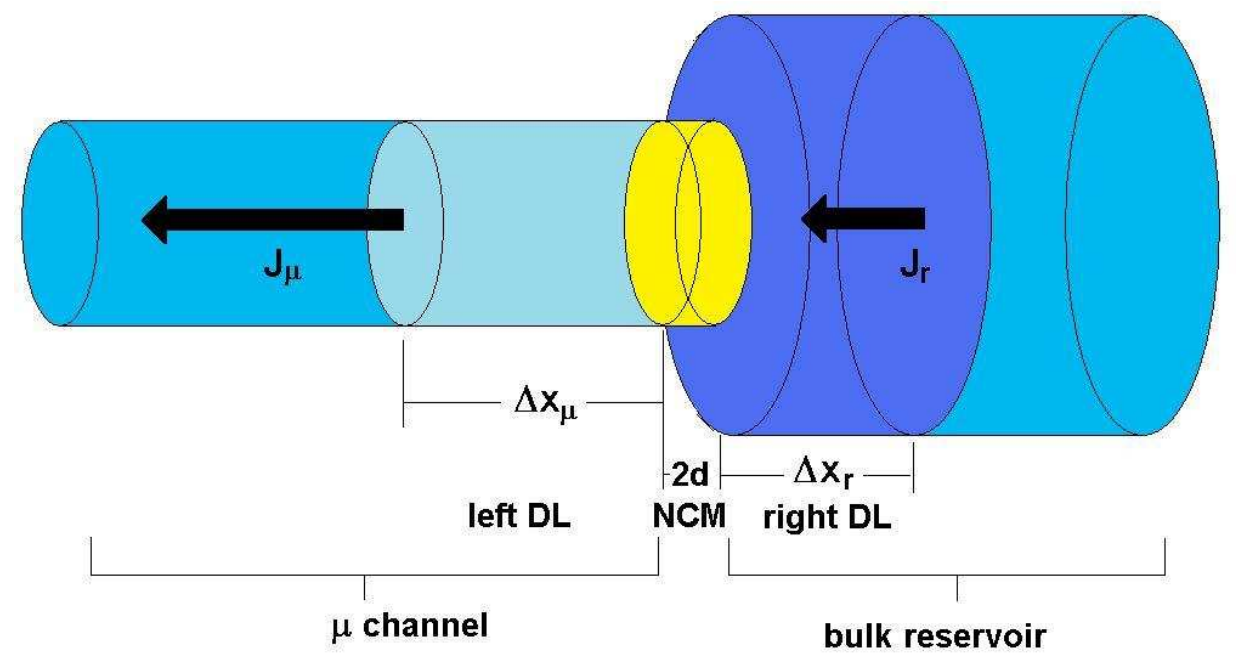

Figure 3.3: An asymmetric discontinuous geometry similar to that in the NCM/NMI.

\subsubsection{Using Hunter's result to compare the DL lengths}

Following this reasoning, we expect the boundaries of the diffusion layer with bulk solution to correspond to the limits $x \rightarrow \pm \infty$. In that limit, the magnitude of the change in reduced concentration from equilibrium near the edge of such a layer is given by

$$
|c| \sim \frac{2 J_{+} t^{3 / 2} \sqrt{\mathcal{D}}}{x^{2}} \exp \frac{-x^{2}}{4 \mathcal{D} t}
$$

The value of the quantity $|c|$ should be the same in the region where the diffusion layer becomes indistinguishable from bulk (making note of the fact that the coordinates have opposite signs in the two half-planes) so that we can compare the estimates for lengths of the layers, $x_{r}=\ell-1$ and $x_{\ell}=L-1$ by equating $\left|c_{r}\right|=\left|c_{\ell}\right|$, or,

$$
\Gamma \equiv \frac{A_{r}}{A_{\mu}}=\left(\frac{L-1}{\ell-1}\right)^{2} \exp \left[\frac{1}{4 \mathcal{D} t}\left((L-1)^{2}-(\ell-1)^{2}\right)\right]
$$

Yossifon and Chang's work demonstrated that the experimentally measured diffusion layer width can be estimated reasonably well by $\Delta x \approx \sqrt{\mathcal{D} t}$ when the system is driven at sub-limiting currents (see Figure 3 in [64].) While it is a crude assumption, this makes it 


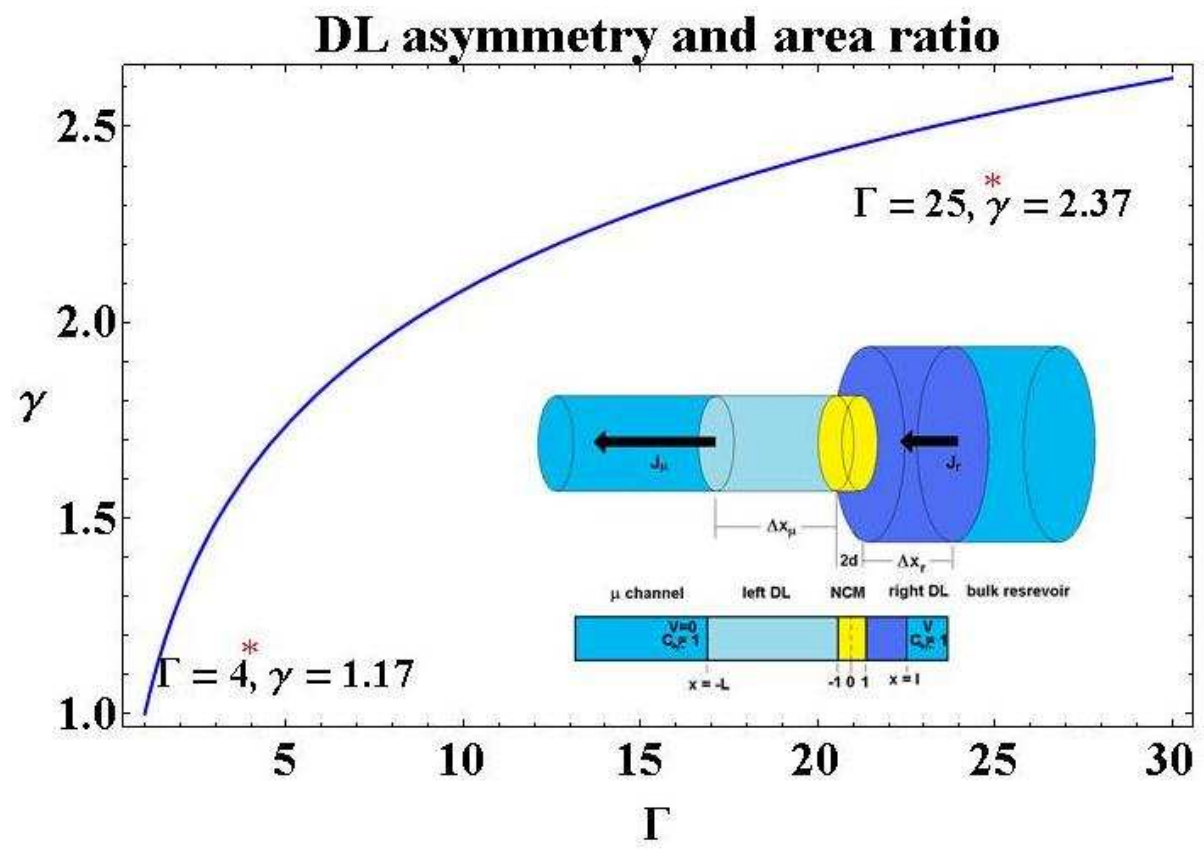

Figure 3.4: A plot of the diffusion-layer length asymmetry versus ratio of cross-sectional areas according to the quasi-1D argument. Data points show values from a preliminary 2D FEM calculation

reasonable to suppose that, in an asymmetric quasi 1D geometry, the diffusion layer lengths may be written as follows:

$$
(L-1)=\gamma \sqrt{\mathcal{D} t}
$$

for the microchannel and

$$
(\ell-1)=\sqrt{\mathcal{D} t}
$$

for the reservoir. Substituting these into equation 3.3.2 yields the time-independent expression,

$$
\Gamma=\gamma^{2} \exp \left[\frac{\left(\gamma^{2}-1\right)}{4}\right]
$$

The hand-waving argument has been tested by preliminary simulation using COMSOL FEM software [3]. This demonstrates that the diffusion layers do grow asymmetrically, with the length asymmetry increasing as the cross-sectional asymmetry increases. Available data is shown along with a plot of the quasi-1D behavior in Figure 3.4. This, and related 
Jarrod E. Schiffbauer Chapter 3. A classical electro-diffusive model of fluidic rectification 60 calculations showing the effects of net fluid flow on diffusion layer growth, as well as an analysis of the Stephan problem of Ref. [49] are planned for future studies [3].

\subsection{Rectification due to asymmetric concentration po- larization using a 1D model}

According to the simple picture of DL dynamics discussed in Sec. 3.3, the diffusion layers in geometrically asymmetric systems can evolve asymmetrically themselves so that, for the NCM/NMI and similar devices, the reservoir DL may be shorter than that in the microchannel at reasonably long times. Aside from the possibility that the diffusion lengths differ, as will be shown subsequently, focusing of the electro-neutral flux through an asymmetric region can result in an essentially similar effect. Thus, quantitative accuracy aside, the straightforward 1D picture will provide some additional insight into how asymmetry in the electro-neutral parts of the concentration polarization layers can cause fluidic rectification.

Provided attention is confined to sub-limiting currents, and the LEN approximation is assumed valid [46], we may use a 1D steady-state electro-diffusive model to investigate the consequences of having asymmetric DL lengths. Formally, the LEN approximation amounts to making the assumption that the Debye layer thickness is negligible. e.g. taking the limit $\epsilon \rightarrow 0$. This approach provides the $0^{\text {th }}$ order approximation to the full electro-diffusive problem (see Ref. [68] and Chapter 5.3 for details) and gives a reasonably good picture of the electro-diffusive processes at applied voltages corresponding to currents below Levich's classical limiting values. It should be emphasized that, even though this model is crude and does not provide a valid picture of DL growth above the classical limiting current [49], the basic notion that changes in cross-section on either side of a perm-selective region can cause asymmetric diffusion layer growth seem reasonable. While the details of this demand further investigation, preliminary work suggests this idea may be qualitatively correct if quantitatively inaccurate. In summary, these models provide a reasonably complete picture of electro-diffusive effects in a simple and intuitive framework which can be used both to un- 
Jarrod E. Schiffbauer Chapter 3. A classical electro-diffusive model of fluidic rectification 61

derstand fluidic rectification in geometrically asymmetric systems and to help explain some of the experimentally observed behavior of the NCM/NMI.

\subsubsection{The 1D LEN model equations}

The steady-state electro-diffusion of ions in a symmetric binary electrolyte is described in $1 \mathrm{D}$ by the dimensionless PNP equations

$$
\mathcal{D}(x)\left[c_{ \pm}^{\prime} \pm z_{ \pm} c_{ \pm} \varphi^{\prime}\right]=-j_{ \pm}
$$

for the species fluxes and Poisson's equation

$$
\epsilon^{2} \varphi^{\prime \prime}=\sum \pm z_{ \pm} c_{ \pm}+N
$$

for the electric potential, and $j_{ \pm}^{\prime}=0$ in the steady-state. $N(x)$ is the (negative) charge density responsible for selectively favoring the passage of counter-ions over co-ions. It is defined piecewise, $N(x)=N$ inside and $N=0$ outside the membrane. The (effective) diffusivity $\mathcal{D}(x)$ is also defined piecewise with $\mathcal{D}(x)=\mathcal{D}$ inside the membrane and $=1$ outside. This allows for a variety of effects which result in an apparent modification of the diffusivity to be bundled into a single constant, which could in principle be defined semiempirically from experimental data or obtained from 2 or 3D simulations.

The concentrations $c_{ \pm}$have been scaled by the value of the bulk concentration $n_{ \pm}$(in $\left(m^{-3}\right)$ ), i.e. $n_{ \pm}(x)=n_{o} c_{ \pm}(x)$ and potentials are scaled by the thermal voltage, $V_{t h}=k T / e$. The dimensionless Debye parameter $\epsilon$ is defined ${ }^{1}$

$$
\epsilon^{2}=\frac{\varepsilon \varepsilon_{o} k T}{4 \pi n_{o} e^{2} \delta^{2}}
$$

The length scale $\delta$ is the (dimensional) membrane half-thickness and these equations are supplemented by boundary conditions at the bulk edges (see Figure 3.5). Restricting attention to the LEN assumption results in the left-side of the Poisson equation being set to zero,

\footnotetext{
${ }^{1}$ This quantity is defined slightly differently, with various choices of length scale or multiplicative constants, depending on the circumstances. For instance, when considering double-layer overlap, the distance scale is often taken as the channel height or radius, whereas here it is defined in terms of a linear dimension. This can be the source of some ambiguity when attempting to interpret estimates, such as those in Refs citeMMM1996, MMM2001, JFM2007 if not considered carefully.
} 
Jarrod E. Schiffbauer Chapter 3. A classical electro-diffusive model of fluidic rectification 62 i.e.,

$$
\epsilon^{2} \varphi^{\prime \prime} \approx 0
$$

The model equations may be integrated to obtain a closed-form solution in this approximation. By substitution of a series expansion of the form

$$
\varphi(x)=\sum_{n=1}^{m} \epsilon^{n} \varphi_{n}
$$

into the Poisson equation, it is apparent that the LEN approximation is equivalent to the leading-order contribution to the full boundary-value problem.

A reduced ion concentration may be defined

$$
c=\frac{c_{+}+c_{-}}{2}
$$

Notice that for a binary electrolyte, this is equivalent to the (electro-neutral) stoichiometric salt concentration.

\subsubsection{Geometry and boundary conditions}

The model geometry used in both ideal and non-ideal membrane models is shown in Figure 3.5. Quantities for each respective region will be denoted by the subscripts $l$ for $-L<x<-1, r$ for $1<x<\ell$ and $m$ for the region inside the membrane, $x \in(-1,1)$. The applied electric potential difference is a known control parameter and indicated in the figure and the concentrations at both $x=-L$ and $x=\ell$ are assumed fixed and set to unity.

In the approximation employed herein (see below,) the regular boundary conditions must be supplemented by additional conditions at the membrane to close the problem. This is because, even though the thickness of the EDL will be neglected, the accompanying jump in electric potential and ion concentration across the Gibbs' plane between the bulklike DL's and the membrane interior must be taken into account. In the steady-state, the electrochemical potential of each species, $\mu_{ \pm}(x)=\ln c_{ \pm}(x) \pm \varphi(x)$, will be continuous across the Gibbs' plane so that we have the four conditions,

$$
\mu_{ \pm, l}(-1)=\mu_{ \pm, m}(-1)
$$




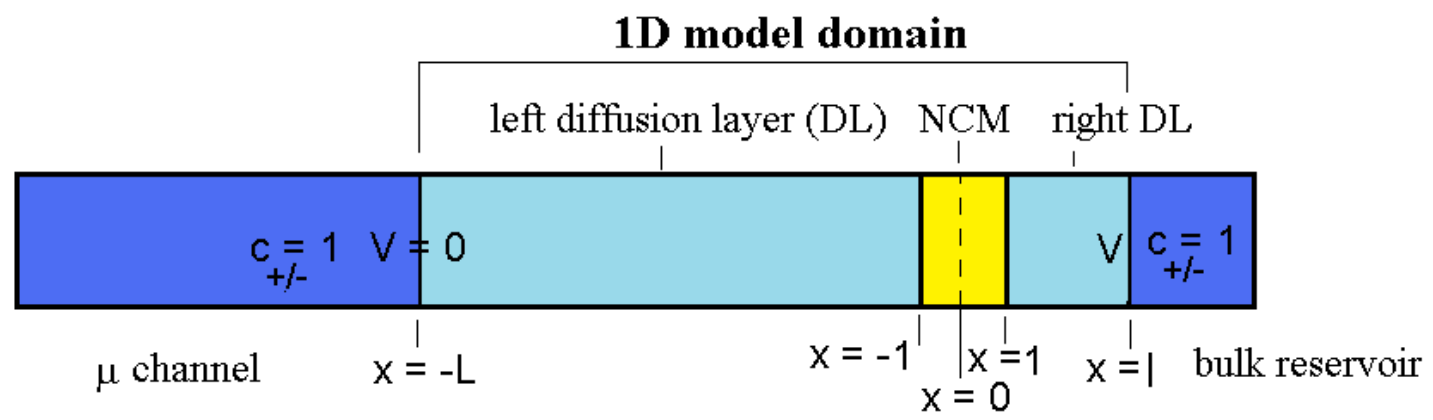

Figure 3.5: Geometry of the model system showing each region

$$
\mu_{ \pm, m}(1)=\mu_{ \pm, r}(1)
$$

\subsubsection{The CP solution in the diffusion layers}

For modest currents and voltages, the ion concentrations in the diffusion layers are approximately equal, so the regions are locally electro-neutral (LEN.) The non-LEN boundary layers are thin, of order $O(\epsilon)$ for sub-limiting currents and may often be neglected in micron and above sized structures at low voltages, effectively setting the left-hand-side of Eqn. 3.4.1 to zero. In this case, the governing PNP equations may be solved for the reduced concentration, $c_{l / r}=\left(c_{+}+c_{-}\right)_{l / r} / 2$ and potential $\varphi_{l / r}$ in each region subject to known boundary conditions at the boundaries between the diffusion layers and bulk at $x=-L$ and $x=\ell$.

The solution algorithm is the same in each region and consists of adding and subtracting the two governing equations coupling the reduced concentrations and potential as in the ideal case. Since we are concerned with the steady-state, $\nabla \cdot I=0$ (where $I$ is the net electric current density) which implies the species flux densities are also continuous throughout the system. For convenience, introduce the following two definitions,

$$
J_{+}=\frac{j_{+}+j_{-}}{2}
$$


Jarrod E. Schiffbauer Chapter 3. A classical electro-diffusive model of fluidic rectification 64 which is the density of the salt flux, and

$$
J_{-}=\frac{j_{+}-j_{-}}{2}
$$

which defines the density of charge flux, so that the net electric current density is given by

$$
I=2 J_{-}
$$

with the sign convention such that a positive cationic flux results when the electric field points towards the right in the system, e.g. flowing down the electric potential gradient.

Left side of system, $-\mathrm{L}<\mathrm{x}<-1$

Here the governing equations become,

$$
c_{l}^{\prime}=-J_{+}
$$

and

$$
c_{l} \varphi_{l}^{\prime}=-J_{-}
$$

Both may be integrated, with the known values at $x=-L$ as the lower limits of integration. This results in

$$
c_{l}(x)=1-J_{+}(x+L)
$$

for the ion concentration profile, and for the electric potential, we obtain

$$
\varphi_{l}(x)=\frac{J_{-}}{J_{+}} \ln \left[1-J_{+}(x+L)\right]
$$

Right side of system, $1<\mathrm{x}<\ell$

The problem on the right hand side of the system is essentially the same, with the governing equations

$$
c_{r}^{\prime}=-J_{+}
$$


Jarrod E. Schiffbauer Chapter 3. A classical electro-diffusive model of fluidic rectification 65

and

$$
c_{r} \varphi_{r}^{\prime}=-J_{-}
$$

Integration yields the solutions for the right side, the only difference being the lower limits of integration are the unknowns and the upper limits are the known values at the right boundary $(x=\ell)$ of the system. So we obtain

$$
c_{r}(x)=J_{+}(\ell-x)+1
$$

for the ion concentration profile, and for the electric potential,

$$
\varphi_{r}(x)=\frac{J_{-}}{J_{+}} \ln \left[J_{+}(\ell-x)+1\right]+V
$$

The changes in concentration and potential across the the membrane must be taken into account. and the continuity of the electro-chemical potential for each species, $\mu_{i}=\ln c_{i}+z_{i} \varphi$, imposed at the membrane-electrolyte boundaries $x=-1$ and $x=1$. However, before doing this, a few points warrant mentioning. Note that the locally electro-neutral approximation breaks down whenever the reduced concentration goes to zero at one of the membrane boundaries. Since it is assumed the diffusion layers are a priori asymmetric, a quick inspection of the above equations suggests that the transport properties of this system inherit this geometric asymmetry.

The fundamental connection between diffusion layer length and the rectification effect may be understood entirely in terms of Levich's classical diffusion-limited current for the asymmetric 1D system without any reference to the details of transport through the membrane itself. Since the concentration in the depleted region is non-negative, a limiting current corresponds to $c \rightarrow 0$. For a system with asymmetric diffusion lengths, there are two different limiting values depending on the direction of the applied field, i.e. depending on which side of the membrane is depleted. Thus, there will be a preferential direction for current transport determined by which side has the higher limiting current density. This can be expressed in terms of the salt flux density in the locally electroneutral (LEN) approximation by evaluating the concentrations immediately outside of the membrane $(x= \pm 1)$. A depleted 
Jarrod E. Schiffbauer Chapter 3. A classical electro-diffusive model of fluidic rectification 66 region forms on the left side of the membrane when the E-field, hence cation (and salt) flux density, are directed towards the right. In this case, the limiting salt flux density is

$$
J_{+, l i m}=\frac{1}{(L-1)}
$$

When the field is directed towards the left, the depleted region forms to the right of the membrane and the limiting flux density is

$$
J_{+, l i m}=\frac{1}{(\ell-1)}
$$

So if the right diffusion layer is longer than the left diffusion layer, the device rectifies with the off-state occurring when the right side is at a lower voltage than the right and the onstate when the right side is at a higher voltage.

Above this limiting current, the LEN approximation breaks down. Physically, the approach to this breakdown is accompanied by the appearance and growth (with increasing current) of a macroscopic extended space charge front in the depleted diffusion region. Mathematically, it corresponds to the increasing importance of higher-order terms and the appearance of an intermediate length scale larger than $O(\epsilon)$. While the appearance of an extended space charge region can reasonably be expected to affect transport, the argument presented here shows that rectification in geometrically asymmetric systems is a leading-order effect.

In principle, this simple explanation may be extended to determine the off- and on- states for any device by comparing the limiting currents. To obtain any more detail, such as the relationship between salt-flux density and electric current density, requires the introduction of a membrane model. Doing so will also provide additional insight into the relationship between diffusion layers and trans-membrane transport, including rectification.

\subsubsection{The ideal membrane case}

Inside the LEN ideal membrane, $-1<x<1$, the reduced concentration is assumed constant and entirely due to cations, $c_{m}(x)=N$, so that the equation for the potential is

$$
\mathcal{D} N \varphi_{m}^{\prime}=-j
$$


Jarrod E. Schiffbauer Chapter 3. A classical electro-diffusive model of fluidic rectification 67

or, integrating,

$$
\varphi_{m}=-\frac{j}{\mathcal{D} N}(x+1)+\varphi_{m}(-1)
$$

The current through the membrane is due to the electrical conduction of cations only, so that $J_{+}=j_{+} / 2$ and $J_{-} / J_{+}=1$ and the \pm subscripts may be omitted without risk of confusion.

Since the electrochemical potential of the anions is equal to zero, we have only the two continuity conditions on the cationic electrochemical potential at each of the solutionmembrane interfaces,

$$
\mu_{\ell}(-1)=\mu_{m}(-1)
$$

and

$$
\mu_{m}(1)=\mu_{r}(1)
$$

Using the known solutions for concentrations in the electrolyte layers along with the concentration and potential in the membrane, the voltage drop across the system can be written as a function of the (cationic) current density,

$$
V=2 \ln \left[\frac{-\frac{j}{2}(L-1)+1}{\frac{j}{2}(\ell-1)+1}\right]-2 \frac{j}{\mathcal{D} N}
$$

Several I-V curves are plotted in figure 3.6 for constant membrane parameters and values of $L$ increasing from symmetric $(L=1)$ to asymmetric $(L=10)$ where the non-dimensional left-hand side diffusion-length is $L-1$. The current-voltage curves in figures 3.6 clearly show current rectification as an effect dependent upon the asymmetry of the diffusion layers. The $\mathrm{I}-\mathrm{V}$ response may be understood by considering the behavior of the terms in the voltage characteristic above. The total voltage drop in the ideal case may be thought of as the sum of an Ohmic part $\Delta V_{m}$, representing the drop across the ideal membrane, and a logarithmic term, $\ln \left[c_{r}(1) / c_{\ell}(-1)\right]$ representing (equivalently) the difference in drops across the diffusion layers, or the osmotic (三chemical) drop imposed across the membrane. $\mathcal{D} N$ may be identified with the membrane conductivity, or equivalently, the membrane resistance, defined as

$$
R_{m}=\frac{2}{\mathcal{D N}}
$$

As the current approaches zero from either the right, i.e. $j \rightarrow 0^{+}$, from an off-state current, or the left, $j \rightarrow 0^{-}$from the on-state, it can be shown that the voltage drop across 
Jarrod E. Schiffbauer Chapter 3. A classical electro-diffusive model of fluidic rectification 68 the membrane is always larger in the on-state than the off-state. Compare two Taylor expansions of the membrane drop $\Delta V_{m}$ term-by-term using the expression $j=2 \alpha /(L-1)$ for the limit $j \rightarrow 0^{-}$(approaching zero from the off-state) and using $j=-2 \alpha /(\ell-1)$ for the on-state limit, with the formal limit $\alpha \rightarrow 0$. Defining the asymmetry factor $\gamma$ as

$$
\gamma=\frac{L-1}{\ell-1}
$$

we may write the logarithmic terms corresponding to the 'chemical drop' across the membrane in each case as,

$$
\ln \frac{c_{r}}{c_{\ell}}=\sum_{n-\text { odd }}^{\infty} \alpha^{n}\left(\gamma^{n}+1\right)+\sum_{m-\text { even }}^{\infty} \alpha^{m}\left(\gamma^{m}-1\right)
$$

for the off-state and for the on-state,

$$
\ln \frac{c_{r}}{c_{\ell}}=-\sum_{n-\text { odd }}^{\infty} \alpha^{n}\left(\gamma^{n}+1\right)+\sum_{m-\text { even }}^{\infty} \alpha^{m}\left(\gamma^{m}-1\right)
$$

Note that if $\gamma=1$, then the $m$-even terms vanish in both series so the membrane voltage drop is symmetric, with sign of the chemical shift opposite the sign of the applied voltage $V$ and equal in both off- and on- states. Otherwise the symmetry is broken; for $\gamma \neq 1$ the on-state series has alternating terms and the off-state series is monotonic. In terms of the potentials, the chemical drop associated with the on-state is less than the chemical rise in the off-state, so the magnitude of $\Delta V_{m}$ is larger in the on-state than the off-state even as the current goes to zero.

The ideal membrane model serves a purely one-dimensional analogue to Wei's dual-cone model [62] and deepens the understanding of the relationship between rectification and CPL asymmetry provided by the classical limiting current arguments. It clearly shows the magnitude of the voltage drop across the membrane or channel depends on the direction of the applied field and that this persists even in the limit of vanishing current. The on-state corresponds to a device orientation with lower resistance (higher conductivity) in the diffusion layers than the off-state. 
Jarrod E. Schiffbauer Chapter 3. A classical electro-diffusive model of fluidic rectification 69

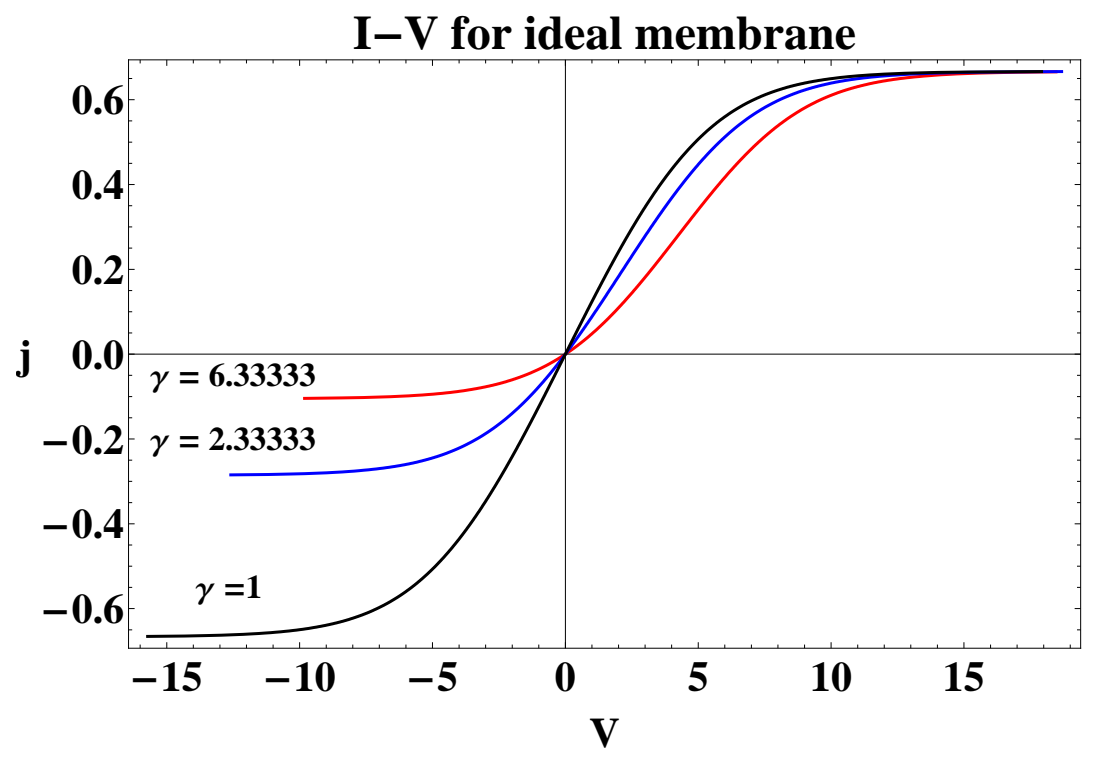

Figure 3.6: Ideal membrane I-V curve showing rectification dependent on DL length asymmetry. $I=-j$ is the dimensionless current density (zero co-ion flux.) The parameters are $N=10, \mathcal{D}=0.1$, and $\ell=1.5$

\subsubsection{The non-ideal membrane case}

For the non-ideal membrane, the electro-neutrality condition is

$$
c_{+}=c_{-}+N(x)
$$

An obvious question, the affirmative answer to which is often taken for granted, is whether or not this condition holds across any reasonably large section of a membrane outside of thin boundary layers. This issue will be examined more rigorously in chapter 4, but for now, the LEN assumption will be employed.

Employing the electro-neutrality condition and the piecewise definition of the fixed charge density, the governing equations are re-written in terms of the reduced concentration. Since in either the left or rightmost regions (subscripts $\ell$ or $r$, ) $c_{+}=c_{-}$, the equations have the same form as the original equations with the diffusivity set to unity. However, in the membrane itself $c_{+}=c+N / 2$ and $c_{-}=c-N / 2$, so instead,

$$
c_{m}^{\prime}+\left(c_{m}+\frac{N}{2}\right) \varphi_{m}^{\prime}=-\frac{j_{+}}{\mathcal{D}}
$$


Jarrod E. Schiffbauer Chapter 3. A classical electro-diffusive model of fluidic rectification 70

$$
c_{m}^{\prime}-\left(c_{m}-\frac{N}{2}\right) \varphi_{m}^{\prime}=-\frac{j_{-}}{\mathcal{D}}
$$

Membrane interior, $-1<\mathrm{x}<1$

In this case, addition of the two governing equations results in

$$
c_{m}^{\prime}+\frac{N}{2} \varphi_{m}^{\prime}=-\frac{J_{+}}{\mathcal{D}}
$$

and subtraction in,

$$
\varphi_{m}^{\prime}=-\frac{1}{c_{m}} \frac{J_{-}}{\mathcal{D}}
$$

Combining these two equations yields

$$
c_{m}^{\prime}-\frac{N J_{-}}{2 \mathcal{D}} c_{m}^{-1}=-\frac{J_{+}}{\mathcal{D}}
$$

or re-arranging for convenience,

$$
c_{m}^{\prime}=\frac{N J_{-}-2 J_{+} c_{m}}{2 \mathcal{D} c_{m}}
$$

This may be integrated directly, giving the following transcendental equation for the ion distribution inside the membrane,

$$
\mathcal{D}\left[c_{m}(x)-c_{m}(-1)\right]+\frac{\mathcal{D} N}{2} \frac{J_{-}}{J_{+}} \ln \left[\frac{c_{m}(x)-\frac{N J_{-}}{2 J_{+}}}{c_{m}(-1)-\frac{N J_{-}}{2 J_{+}}}\right]=-J_{+}(x+1)
$$

Given the solution to this equation, the potential may be found,

$$
\varphi_{m}(x)=-\frac{J_{-}}{\mathcal{D}} \mathfrak{F}(x)+\varphi_{m}(x)
$$

where the integral

$$
\mathfrak{F}(x)=\int_{-1}^{x} c_{m}^{-1}\left(x^{\prime}\right) d x^{\prime}
$$

has been introduced.

Here the left membrane boundary is taken as the lower limit of integration, with the variable $x$ and the functions $c_{m}(x)$ and $\varphi_{m}(x)$ as the upper limits. This could have just as easily been done in the opposite fashion. The values at both boundaries are unknowns which must be determined by applying the continuity conditions and employing the known 
Jarrod E. Schiffbauer Chapter 3. A classical electro-diffusive model of fluidic rectification 71

solutions from the left and right regions of the model. Also notice the appearance of the membrane conductivity. However, as will be apparent later on, it cannot be considered as the single parameter governing the membrane response nor will the diffusion layer conductivity be independent of membrane charge in the non-ideal membrane.

\section{Application of continuity conditions, exact current-voltage relationship}

The continuity conditions at the membrane surfaces (Eqns. ??) are written out explicitly below for each species at each boundary.

The continuity condition for cation electrochemical potential at $x=-1$ is,

$$
\ln c_{\ell}(-1)+\varphi_{\ell}(-1)=\ln \left[c_{m}(-1)+\frac{N}{2}\right]+\varphi_{m}(-1)
$$

and for the anions,

$$
\ln c_{\ell}(-1)-\varphi_{\ell}(-1)=\ln \left[c_{m}(-1)-\frac{N}{2}\right]-\varphi_{m}(-1)
$$

At the $x=1$ boundary, the cation condition is

$$
\ln \left[c_{m}(1)+\frac{N}{2}\right]+\varphi_{m}(1)=\ln c_{r}(1)+\varphi_{r}(1)
$$

and for the anions,

$$
\ln \left[c_{m}(1)-\frac{N}{2}\right]-\varphi_{m}(1)=\ln c_{r}(1)-\varphi_{r}(1)
$$

Combining the first two equations yields

$$
c_{m}^{2}(-1)-\frac{N^{2}}{4}=c_{\ell}^{2}(-1)
$$

and evaluating the left-side solution (Eqn. [?]) at $x=-1$, we obtain an expression for the value of the membrane concentration at the left membrane boundary,

$$
c_{m}(-1)=\sqrt{\left[1-J_{+}(L-1)\right]^{2}+N^{2} / 4}
$$

The second pair of equations may combined to give a similar condition holding at the right membrane boundary,

$$
c_{r}^{2}(1)=c_{m}^{2}(1)-\frac{N^{2}}{4}
$$


Jarrod E. Schiffbauer Chapter 3. A classical electro-diffusive model of fluidic rectification 72 or, evaluating Eqn. 3.4 .3 at $x=1$,

$$
c_{m}(1)=\sqrt{\left[J_{+}(\ell-1)+1\right]^{2}+N^{2} / 4}
$$

Using the expressions for the potentials at the left and right solution-side of the respective membrane surfaces, i.e. $\varphi_{r}(1)$ and $\varphi_{\ell}(-1)$, combining the equations for continuity of cation electrochemical potentials (Eqns. ??,) and eliminating the potential $\varphi_{m}(-1)$ in the process, the following equation for the current-voltage relationship is obtained,

$$
V=\ln \left[\frac{c_{m}(1)+N / 2}{c_{m}(-1)+N / 2}\right]-(Q+2) \ln \left[\frac{c_{r}(1)}{c_{\ell}(-1)}\right]-\frac{J_{+}(Q+1)}{\mathcal{D}} \mathfrak{F}(1)
$$

Here we have introduced the parameter $Q$, defined through

$$
\frac{J_{-}}{J_{+}}=Q+1
$$

Two additional pieces are required from the solution of equation for $c_{m}(x)$ to obtain the explicit current-voltage relationship. The integral $\mathfrak{F}(1)$ must be evaluated and the solution for $Q$ in terms of $J_{+}$obtained using the expressions in equations ?? for the ion concentration at the membrane edges. The exact solutions of the transcendental equation and its integral require numerical evaluation. However approximate analytic solutions are also available. The various approximations are discussed in the following section, along with the results for the limit of large $N$, which is intended to approach the ideal membrane case.

After evaluating the transcendental equations for $c_{m}(x)$ and for $Q\left(J_{+}\right)$numerically, the current-voltage characteristic may be obtained. This is plotted in figure ?? for several values of $L$, these are indicated in the inset along with the selectivity as a function of voltage for each value. The selectivity is related to $Q$, i.e. defining the membrane selectivity as

$$
\beta=j_{+} / j_{-}=\frac{1+Q}{1-Q}
$$

This quantity, or equivalently, $Q$, is useful in understanding the behavior of a non-ideal perm-selective membrane.

In the non-ideal case, the total current is given by $I=-2 \mathcal{D} c_{m} \varphi_{m}^{\prime}$ so even though the concentration profiles inside the membrane are no longer exactly linear, the potential drop across the membrane can still be used to qualitatively understand the behavior of the nonideal membrane rectifier. To facilitate the discussion of these results in comparison with the 


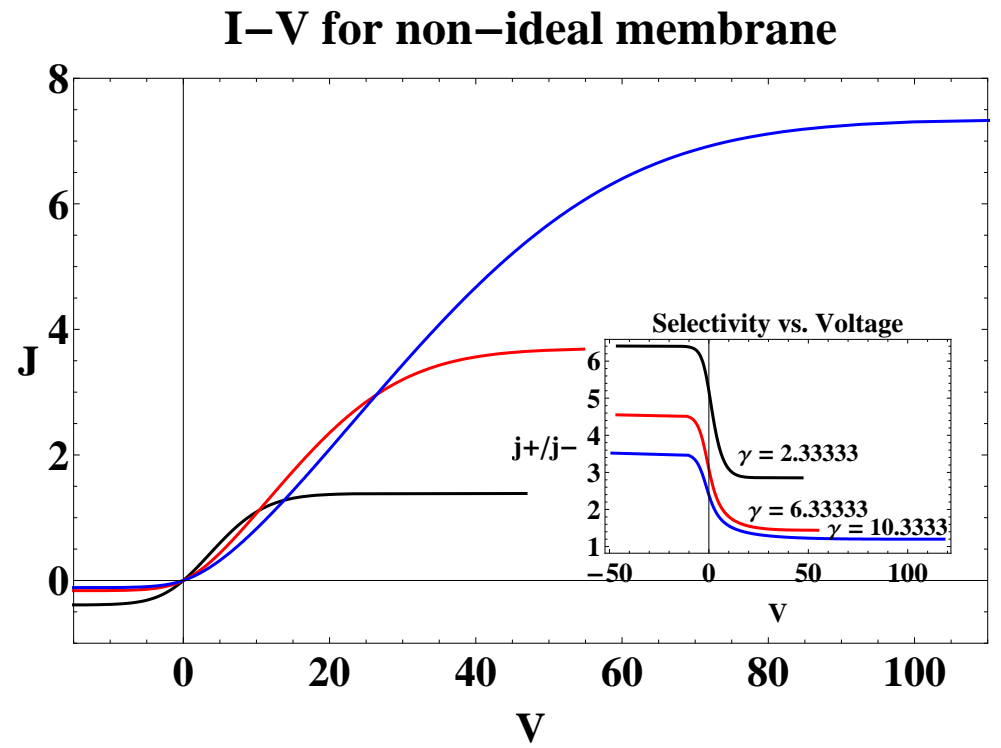

Figure 3.7: Non-ideal membrane IV curve for systems with several values of asymmetry $\gamma$, and fixed charge density $N=10$, effective diffusivity $\mathcal{D}=0.2$, and $\ell=1.5$. The variation of selectivity with voltage, along with a legend for the asymmetry values, is shown in the inset.

ideal case, it is useful to go back to the continuity equations and write expressions for the voltage drop across the membrane, $\Delta V_{m}$, in both cases and compare. These expressions are essentially equivalent to the current-voltage equations, with

$$
\Delta V_{m}=2 \ln \frac{c_{\ell}(-1)}{c_{r}(1)}+V
$$

for the ideal membrane and

$$
\Delta V_{m}=Q \ln \frac{c_{\ell}(-1)}{c_{r}(1)}+V
$$

for the non-ideal membrane. Since $Q \leq 1$ for a cation-selective membrane, the above expressions suggest the non-ideal membrane will generally carry more current than the ideal membrane. This may be anticipated also because there are now charge carriers of both sign inside the membrane. While the off-state response is essentially similar in the ideal and nonideal cases, aside from a generally higher overall current due to the anion flux, the on-state classical limiting current increases with increasing asymmetry in the system. This result can be expected to influence the behavior in asymmetric systems even above the classical limit. 
Jarrod E. Schiffbauer Chapter 3. A classical electro-diffusive model of fluidic rectification 74

\section{Near-limiting CP and $\phi$ vs. $x$}

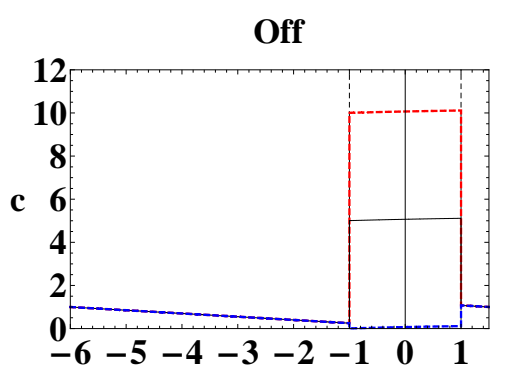

$\mathbf{x}$

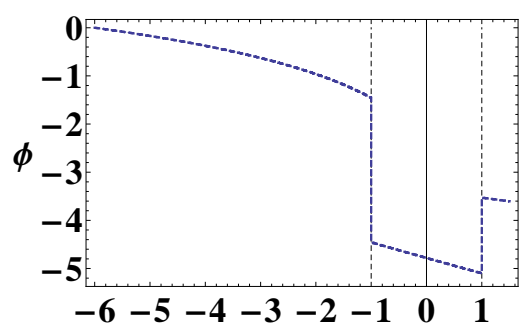

$\mathbf{X}$

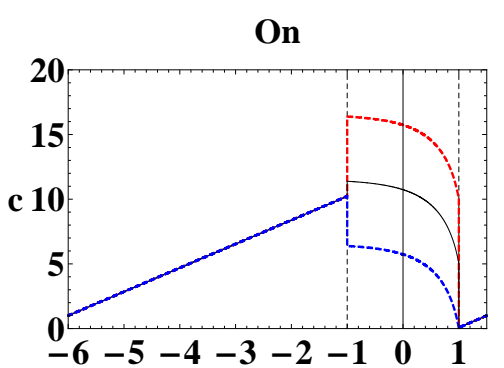

$\mathbf{X}$

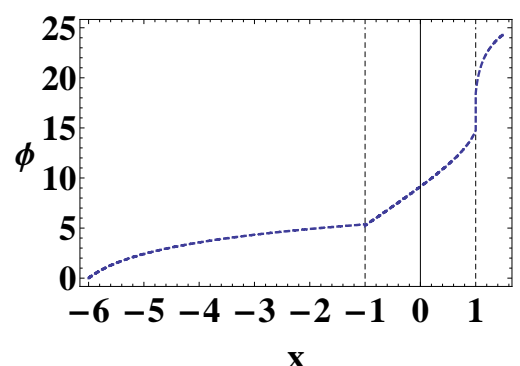

$\mathbf{x}$

Figure 3.8: Concentration (top) and electric potential (bottom) profiles for the off- and onstates near the limiting current for the same parameter values as the previous plot, with $\gamma=6.33$. The cation concentration is shown in red, the anion concentration in blue and the reduced (salt) concentration in black. The membrane edges are denoted by vertical lines at $x= \pm 1$.Note the strong concentration polarization in the on-state CP profile and the ratchet- like potential in the off-state. 
Jarrod E. Schiffbauer Chapter 3. A classical electro-diffusive model of fluidic rectification 75

\subsubsection{Limit of large $\mathrm{N}$ for non-ideal membrane}

Higher charge fixed charge density leads to higher perm-selectivity since more co-ions are excluded. Thus we expect there to be some limit in which the charge density increases and the non-ideal membrane behaves similarly to the ideal membrane. The low-voltage behavior of the two models bears this out. However, the correspondence between the two models can be studied further and is not quite as straightforward as simply finding an asymptotic expression for the voltage-current relationship for very large $N$. The formal limit $N \rightarrow \infty$ is not taken. Instead, because the current must remain finite and non-zero for a finite non-zero voltage, the product $\mathcal{D} N$ must also remain finite if this limit were to be imposed formally. This implies that $\mathcal{D}=O(1 / N)$ and hence vanishes in the limit of infinite fixed charge density. Therefore we will take the product $\mathcal{D} N$ to be a constant of order unity.

One more ingredient is required to find this limiting value; bounds must be placed on the various terms in the equation governing $c_{m}(x)$. Consider the first (linear) term in equation 3.4.5,

$$
\mathcal{D}\left[c_{m}(x)-c_{m}(-1)\right]
$$

Since this difference will be greatest at the ends, or formally,

$$
\max \left|c_{m}(x)-c_{m}(-1)\right| \leq\left|c_{m}(1)-c_{m}(-1)\right|
$$

we can evaluate the order of the right-hand side of the above inequality to place bounds upon the linear term in equation 3.4.5. This is evaluated by Taylor-expanding the radicals in equations ?? and looking at the difference of the lowest-order (non-constant) terms.

$$
c_{m}(1)=\frac{N}{2}\left[1+\frac{1}{2}\left(\frac{4\left[J_{+}(\ell-1)+1\right]^{2}}{N^{2}}\right)+O\left(N^{-4}\right)\right]
$$

and

$$
c_{m}(-1)=\frac{N}{2}\left[1+\frac{1}{2}\left(\frac{4\left[1-J_{+} \gamma(\ell-1)\right]^{2}}{N^{2}}\right)+O\left(N^{-4}\right)\right]
$$

Notice that the expansions are about small $N^{2}$, however the difference itself is of order $O(1 / N)$. The resulting order of the linear term from equation 3.4 .5 is then $O\left(1 / N^{2}\right)$, consistent with the Taylor expansions. Thus this term may be neglected in the limit $N \rightarrow \infty$ and 
Jarrod E. Schiffbauer Chapter 3. A classical electro-diffusive model of fluidic rectification 76 efforts focused on linearizing the logarithmic term, the argument of which is indeterminate. Exponentiating both sides of the remaining terms in equation 3.4.5 yields,

$$
\frac{c_{m}(x)-\frac{N J_{-}}{2 J_{+}}}{c_{m}(-1)-\frac{N J_{-}}{2 J_{+}}}=\exp \left[\frac{-2 J_{+}^{2}}{\mathcal{D} N J_{-}}(x+1)\right]
$$

This may be solved immediately for $c_{m}(x)$ using the approximation for $c_{m}(-1)$ and the quantity $Q$. However, in the limit of large $N$, the fluxes $J_{+} \rightarrow J_{-}$so that $Q \ll 1$, in particular $Q=O\left(N^{-2}\right)$ as will be shown subsequently, so that to leading-order,

$$
\frac{1}{Q+1} \approx 1
$$

and thus, for $c_{m}(x)$ we may write

$$
c_{m}(x)=\left[\frac{\left[1-J_{+} \gamma(\ell-1)\right]^{2}}{N}-\frac{N}{2} Q\right] \exp \left[-\frac{2 J_{+}}{\mathcal{D} N}(x+1)\right]+\frac{N}{2}(Q+1)
$$

Two more steps are required to obtain the current-voltage relations for the limit of large $N$; the above equation must be integrated to find the function $\mathfrak{F}(1)$ and (separately) it must be evaluated at $x=1$ and re-arranged to solve for $Q$ as a function of $J_{+}$. First, the latter step is accomplished by evaluating the equation and using the expansion for $c_{m}(1)$ found previously. After some manipulation, this yields the following expression for $Q$,

$$
Q=\frac{2}{N^{2}} \frac{\left[J_{+}(\ell-1)+1\right]^{2}-\left[1-J_{+} \gamma(\ell-1)\right]^{2} \exp \left(-\frac{4 J_{+}}{\mathcal{D} N}\right)}{1-\exp \left(-\frac{4 J_{+}}{\mathcal{D} N}\right)}
$$

which is $O\left(N^{-2}\right)$ as advertised.

The integration in equation 3.4 .5 is greatly facilitated by re-writing $c_{m}(x)$ slightly (a nonessential step, but less cumbersome in terms of notation,) as

$$
c_{m}(x)=\theta e^{\kappa x}+\xi
$$

where the quantities appearing above are temporarily defined

$$
\begin{gathered}
\theta=\left\{\frac{\left[1-J_{+} \gamma(\ell-1)\right]^{2}}{N}-\frac{N}{2} Q\right\} e^{\kappa} \\
\kappa=-\frac{2 J_{+}}{\mathcal{D} N}
\end{gathered}
$$


Jarrod E. Schiffbauer Chapter 3. A classical electro-diffusive model of fluidic rectification 77

and

$$
\xi=\frac{N}{2}(Q+1)
$$

Thus, the integral is

$$
\mathfrak{F}(x)=\int_{-1}^{x} \frac{d x^{\prime}}{\theta e^{\kappa x^{\prime}}+\xi}
$$

The substitution $y=c_{m}(x)$ is then be used, so that

$$
d x^{\prime}=\frac{1}{\kappa(y-\xi)} d y
$$

and the integral may be evaluated in a straightforward way. Keeping in mind that $1 /(Q+1) \approx$ 1 , and evaluating the upper limit at $x=1$, we arrive at the following expression,

$$
\mathfrak{F}(1)=\frac{4}{N}+\frac{\mathcal{D}}{J_{+}} \ln \left\{\frac{\left[\left[1-J_{+} \gamma(\ell-1)\right]^{2}-\frac{N^{2}}{2}\right] e^{-\frac{4 J_{+}}{\mathcal{D} N}+\frac{N^{2}}{2}(Q+1)}}{\left[1-J_{+} \gamma(\ell-1)\right]^{2}+\frac{N^{2}}{2}}\right\}
$$

Finally, we re-write equation 3.4 .5 for the voltage using the appropriate approximate forms for the concentrations to obtain,

$$
V=\ln \left\{\frac{N+\left[J_{+}(\ell-1)+1\right]^{2} / N^{2}}{N+\left[1-J_{+} \gamma(\ell-1)\right]^{2} / N^{2}}\right\}-(Q+2) \ln \left\{\frac{J_{+}(\ell-1)+1}{1-J_{+} \gamma(\ell-1)}\right\}-\frac{J_{+}(Q+1)}{\mathcal{D}} \mathfrak{F}(1)
$$

Notice that the voltage drop across the system is now a sum of four terms (breaking $\mathfrak{F}$ into two parts.) There is an Ohmic part, $4(Q+1) J_{+} / \mathcal{D} N$, and a logarithmic term depending only on the concentrations at the solution-membrane interface. These are completely analogous to the terms in the ideal case. However note the appearance of two additional terms in the non-ideal case that depend on not only the membrane conductivity $\mathcal{D} N$, but also on the membrane charge density itself. Also note the appearance of the $N^{-2}$ pre-factor in the expression for $Q$ (equation 3.4.6,) so that even though the forms of the two terms corresponding to the Ohmic drop and diffusion layer drop appear similar to the ideal case, they too depend on the membrane charge density.

\section{Comparison of ideal and non-ideal membranes}

If we consider a fixed diffusion length $L$ on the left side of the system, and keep the conductivity constant while varying only the membrane charge density, we see that as the 


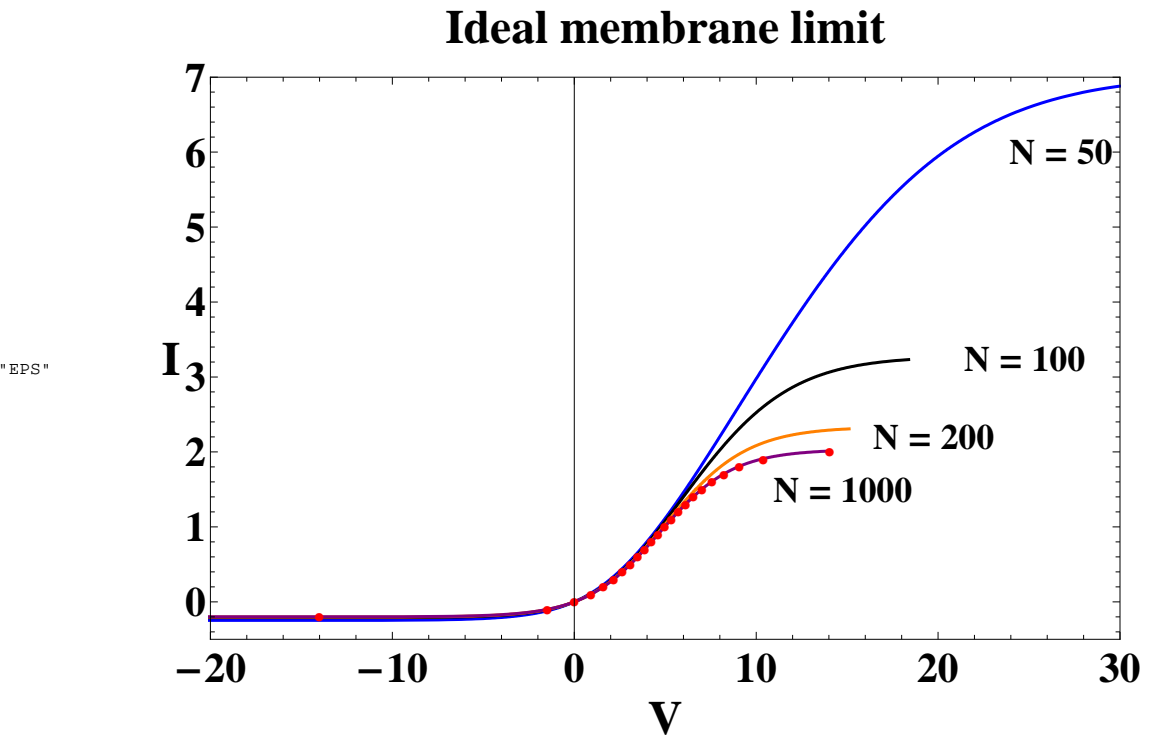

Figure 3.9: Non-ideal cases (solid lines) for several values of membrane charge density $N$ $\gamma=6.3333$, and $\ell=1.5$ and adjusting the membrane diffusivity to keep the product $\mathcal{D} N=1$. For comparison, the red plot shows the ideal membrane case for the same conductivity

charge density increases, the non-ideal behavior approaches that of the ideal case (see Fig. 3.9 below.)

In general, the trend is that less ideal membranes with a high degree of left-right asymmetry in the diffusion layers will tend to have low values of the limiting current in the off-state, comparable to an ideal membrane with a similar degree of asymmetry and the same membrane conductivity $\sigma_{m}$. However, the on-state limiting current along with the voltage corresponding to the knee of the curve, is dramatically increased with increasing asymmetry and decreasing membrane charge density.

In the limit of large $N$, the non-ideal case shares some general trends with the ideal case. In particular, the low-voltage behavior and off-state limiting current appear to more-or-less coincide. One noticeable difference, however, is that the on-state limiting current increases significantly with increasing asymmetry in the left-right diffusion lengths, and this effect depends on membrane charge. As noted previously, in the ideal case, the membrane charge itself is not an independent parameter; it is the product $\sigma_{m}=\mathcal{D} N$ - the membrane con- 
Jarrod E. Schiffbauer Chapter 3. A classical electro-diffusive model of fluidic rectification 79 ductivity - that determines the low-voltage response. Thus in the ideal case, the on-state limiting current depends only on the membrane thickness and the off-state current only on the degree of asymmetry. However, in the non-ideal case, the varying ratio of salt to charge flux density, related to the parameter $Q$, de-couples the charge density and diffusivity - which makes sense physically, as one might anticipate the charge density to regulate the ratio of cationic to anionic current independently of the diffusivity (assuming a symmetric binary electrolyte.)

\subsubsection{Ohmic behavior in the low-voltage limit}

In chapter 2 , it was stated that the low-voltage response is expected to be linear, and that the solution of the Poisson-Boltzmann equation could in principal be used to relate the surface properties of the channel to the channel resistance and perm-selectivity for very near equilibrium conditions. The voltage drop across an ideal membrane can be approximated by assuming that as $V_{a p p} \rightarrow 0, j \rightarrow 0$ so that

$$
V\left(j_{+}\right)=\left[(2+L-\ell)-\frac{2}{D_{m} N}\right] j
$$

So, at low voltage, the total voltage is indeed Ohmic and given as the sum of the drop across the two diffusion layers, i.e. the first linear term, and the drop across the membrane.

\section{Low-voltage limit for the non-ideal membrane}

The non-ideal membrane response is also linear at low voltage, though the dependence

on membrane parameters and diffusion lengths is not as simple as the ideal case. First the logarithmic parts of the potentials in the left and right regions (Eqns. ??) by assuming that $J_{+} \rightarrow 0$ as $V \rightarrow 0$ should be linearized obtaining

$$
\varphi_{\ell} \approx-J_{+} G(x+L)
$$

and

$$
\varphi_{r} \approx J_{+} G(1-x)+V
$$


Jarrod E. Schiffbauer Chapter 3. A classical electro-diffusive model of fluidic rectification 80

where here we have introduced a new parameter (not small) $G$ that is analogous to $Q$ in the large- $N$ limit.

$$
G=\frac{J_{-}}{J_{+}}
$$

We will also require expansions for the concentrations at the membrane boundaries for small $J_{+}$, these are given by

$$
c_{m}(1) \approx \frac{N}{2}\left[\lambda+\frac{4(\ell-1)}{\lambda N^{2}} J_{+}\right]
$$

and

$$
c_{m}(-1) \approx \frac{N}{2}\left[\lambda-\frac{4(L-1)}{\lambda N^{2}} J_{+}\right]
$$

where the parameter

$$
\lambda=\sqrt{\frac{4}{N^{2}}+1}
$$

has been introduced for the sake of convenience. Note that it may be desirable in some circumstances to truncate these even further and use only the leading-order terms to keep the final results linear for small $J_{+}$. This point will be emphasized when appropriate.

By substituting the above directly into equation 3.4.5, evaluated at $x=1$ and keeping terms linear in $J_{+}$, we obtain an expression for the ratio $G$ independent of the flux,

$$
G=\lambda-\frac{\mathcal{D}(\gamma-1)(\ell-1)}{N}
$$

This is compatible with the assumption that, at very low voltages and hence currents well below the limiting current, the applied voltage does not distort the double-layer. Thus a quasi-equilibrium ratio of cations to anions should determine the ratio of cationic to anionic flux, dependent only on the membrane charge and thickness. This result will permit a direct comparison of the $1 \mathrm{D}$ non-ideal membrane transport models here to so-called classical transport models for nanopores obtained using solutions of the Poisson-Boltzmann equation for a nano-pore geometry.

By employing the above expressions and returning to the continuity conditions for electrochemical potentials, essentially following the same general procedure as before and using the above expressions, the voltage $V$ may be written as a function of $J_{+}$as follows,

$$
V=\ln \left\{\frac{\lambda+4 J_{+}(\ell-1) / \lambda N^{2}}{\lambda-4 J_{+} \gamma(\ell-1) / \lambda N^{2}}\right\}+\ln \left[\frac{1-J_{+} \gamma(\ell-1)}{J_{+}(\ell-1)+1}\right]-\left[\mathcal{D}^{-1} \mathfrak{F}(1)+(\gamma-1)(\ell-1)\right] G J_{+}
$$


Jarrod E. Schiffbauer Chapter 3. A classical electro-diffusive model of fluidic rectification 81

In the above expressions, the appropriate asymptotic forms of the logarithms and the function $\mathfrak{F}(1)$ must be obtained. The Taylor expansion of the logarithms is straightforward, with

$$
\ln \left\{\frac{\lambda+4 J_{+}(\ell-1) / \lambda N^{2}}{\lambda-4 J_{+} \gamma(\ell-1) / \lambda N^{2}}\right\} \approx \frac{4(\gamma+1)(\ell-1)}{\lambda N^{2}(\lambda+1)} J_{+}
$$

and

$$
\ln \left[\frac{1-J_{+} \gamma(\ell-1}{J_{+}(\ell-1)+1}\right] \approx-(\gamma+1)(\ell-1) J_{+}
$$

The asymptotic treatment of $\mathfrak{F}$ requires careful consideration. First, we must return to the original expression for $c_{m}(x)$, but making a substitution in a similar spirit to that utilized in linearizing the same equation for large $N$. The reasoning behind the argument in this case is slightly different, though the same sort of formal constraint expressed in inequality 3.4.6 still holds. But in this case, $O\left(N^{-1}\right)$ is not necessarily small, though in principle it could be for large enough $N$. The salient point is the difference $C(x)=c_{m}(x)-c_{m}(-1)$ is at least $O\left(J_{+}\right)$for arbitrary $N$, which is what we require. A more intuitive and physical explanation is based on the fact that the system is close to equilibrium and the concentration across the membrane is practically constant in equilibrium, excepting of course the doublelayers at the interface (which we are ignoring in this approximation anyway.) This intuitive argument is demonstrated by the solution of the Poisson-Boltzman equation for the finitelength capillary case [54]. In any event, though we cannot neglect the term $\mathcal{D} C(x)$, we can expand the logarithm about small $C(x)$, keeping first-order terms. However, note that within this approximation, to be consistently first-order in $J_{+}$it will be necessary to use the (constant) leading-order expression for $c_{m}(-1)$ in subsequent parts of the calculation.

Re-writing the equation in terms of the difference $C(x)$ keeping things (for simplicity) in terms of $c_{m}(-1)$,

$$
\mathcal{D} C(x)+\frac{\mathcal{D} N}{2} G \ln \left[\frac{\left(C(x)-c_{m}(-1)\right)-\frac{N}{2} G}{c_{m}(-1)-\frac{N}{2} G}\right]=J_{+}(x+1)
$$

To first order in $C(x)$, this is just

$$
\mathcal{D} C(x)+\frac{\mathcal{D} N}{2} G\left[\frac{C(x)}{c_{m}(-1)-\frac{N}{2} G}\right]=J_{+}(x+1)
$$

or, re-arranging and solving for $c_{m}(x)$,

$$
c_{m}(x)=\Omega(x+1)+c_{m}(-1)
$$


Jarrod E. Schiffbauer Chapter 3. A classical electro-diffusive model of fluidic rectification 82 where we have introduced another symbol for convenience,

$$
\Omega=\frac{J_{+}\left(c_{m}(-1)-\frac{N}{2} G\right)}{\mathcal{D} c_{m}(-1)}
$$

Note that in the subsequent integration, as mentioned previously, we will use only the leadingorder contribution to $c_{m}(-1)$, or

$$
c_{m}(-1) \approx \frac{N}{2} \lambda
$$

Upon carrying out the integral in equation 3.4 .5 , we obtain

$$
\mathfrak{F}(1)=\frac{\mathcal{D}}{\Omega} \ln \left[\frac{2 \Omega}{\mathcal{D} c_{m}(-1)}+1\right]
$$

After expanding the logarithm, using the above approximation for $c_{m}(-1)$, we have

$$
\mathfrak{F}(1)=\frac{4}{N \lambda}
$$

Putting all of the above results together, we finally arrive at an expression for the voltage as a function of current valid for very low voltages,

$$
V=\left\{\frac{4(\gamma+1)(\ell-1)}{\lambda N^{2}(\lambda+1)}-(\gamma+1)(\ell-1)-\left[\frac{4}{\mathcal{D} N \lambda}+(\gamma+1)(\ell-1)\right] G\right\} J_{+}
$$

Notice again the appearance of an Ohmic membrane resistance term as well as the typical conductance terms for the diffusion layer, i.e. those involving the quantity $(\gamma+1)(\ell-1)$. However, unlike the ideal case, this quantity depends on the explicitly on the membrane charge separately from the membrane resistance term. Note also that in the limit $N \rightarrow \infty$ both $\lambda$ and $G$ approach unity while $J_{+} \rightarrow j_{+} / 2$; thus the above expression approaches the low-voltage limit for the ideal case.

\section{Low-voltage rectification factor}

The rectification factor, defined as $R(V)=\|I(V) / I(-V)\|$ must generally be obtained numerically in the course of obtaining the I-V curves from the function $V\left(J_{+}\right)$. However, the above analysis permits the leading order contribution to the rectification factor to be determined by inspection, assuming locally electro-neutral membrane and solution layers. 
Jarrod E. Schiffbauer Chapter 3. A classical electro-diffusive model of fluidic rectification 83

Since the leading-order selectivity function, $G$, does not depend on the voltage, the lowvoltage limit yields $R_{\text {low v }}=1$. This limit will be shown to correctly estimate the order of magnitude of experimentally determined rectification factors in the in the next chapter.

\subsection{Including flux-focusing effects}

The purely 1D models illustrate the basic principles behind fluidic rectification due to asymmetric concentration polarization, and as was shown in section 2.3 , asymmetric geometries can induce asymmetric diffusion lengths. However, the modeling work of Liu et. al. [30] and the experiments of Yossifon and Chang [65] demonstrate that it is not necessary that the DL's have different lengths in order to rectify. Therefore, it would be desirable to consider simply the effects of flux-focusing on the symmetry of the DL's, disregarding any length asymmetry. It would be reasonable to suppose both effects act in any real system, to a greater or lesser degree. By simply re-scaling the current density in the reservoir region, some idea of the relative strength of the flux-focusing effect compared to (1D) diffusion layer length asymmetry can be gained without making any radical changes in the modeling approach. In this case, the reservoir concentration profile is given by

$$
c_{r}(x)=\frac{J_{+}}{\Gamma}(\ell-x)+1
$$

Then the corresponding terms in equation 3.4.5 must also be modified.

In figure 3.10 the resulting I-V characteristics are plotted for a number of values of $\Gamma$, the cross-sectional asymmetry (with $\gamma=1$ ) and these are compared to the pure 1D model with a range of $\gamma$ corresponding to the range of $\Gamma$ used in the flux-focused calculations. The parameter values are the same as those used in figure 3.7. The overall qualitative behavior of the two versions of the model as a function of the relevant asymmetry parameter is comparable. While the on-state current density is higher for the flux-focused system, the knee voltages appear to be very similar. The low-voltage and off-state currents in the flux-focused cases are practically indistinguishable while the 1D DL asymmetry models show a visible decrease in the magnitude of the low-voltage and off-state current with increasing asymmetry. The rectification factor, defined here as the ratio of on- to off- state current density is slightly 
Jarrod E. Schiffbauer Chapter 3. A classical electro-diffusive model of fluidic rectification 84 lower for the flux-focused cases, but is otherwise similar. The selectivity shows the greatest differences between the two models. The flux-focused model shows a higher selectivity value, about a factor of two, and it is not entirely clear why this is the case from the analysis here. It might be interesting to delve into the matter further. Overall, it would be very difficult to distinguish between the two cases experimentally from examining the I-V curves alone, and it is clear that the general conclusion that devices with greater asymmetry are stronger rectifiers with higher limiting on-state currents and knees at higher voltages applies equally well to both cases. 2D numerical studies could provide an interesting and more realistic comparison between the two types of asymmetry. Calculations with a DL model using finite length conical regions, similar to the approach of Wei et. al. [62], with the non-ideal membrane model could be interesting as well.

\section{Variation of bulk concentration}

Since the concentrations-hence charge densities including the fixed $N$ - are scaled by the bulk concentration, varying the bulk concentration is equivalent to varying the membrane charge density $N$ in the above model. Thus, high values of $N$ correspond to relatively low values of bulk concentration and visa versa. A general trend is that higher charge density in the membrane corresponds to a lower overall value of the limiting current density. This is shown using both the 1D asymmetric DL and flux-focused model (with $\gamma=1$ ) in figure 3.11. Again, the on-state currents are generally higher for the flux-focused model. This may be understood, at least in a hand-waving fashion, by considering that the resistance of a conductor goes as the inverse of the cross-section. At low (positive) voltages, the slopes of the low concentration/high charge density curves are higher than the high concentration/low $N$ slopes. The rectification factors for the two cases are almost identical, with the flux-focused value slightly lower than the $1 \mathrm{D}$ value. The selectivity, again, shows the greatest difference; in this case, the flux-focused values are about an order of magnitude greater and vary over an order of magnitude with voltage as compared to the 1D model. Further study of this 
Jarrod E. Schiffbauer Chapter 3. A classical electro-diffusive model of fluidic rectification 85

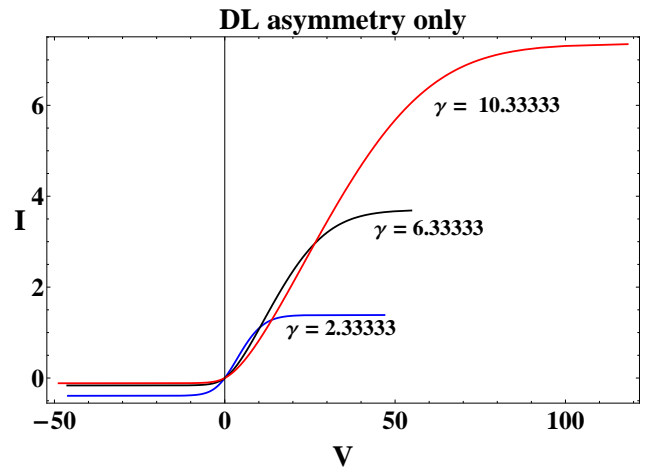

Rectification factor for DL asym.

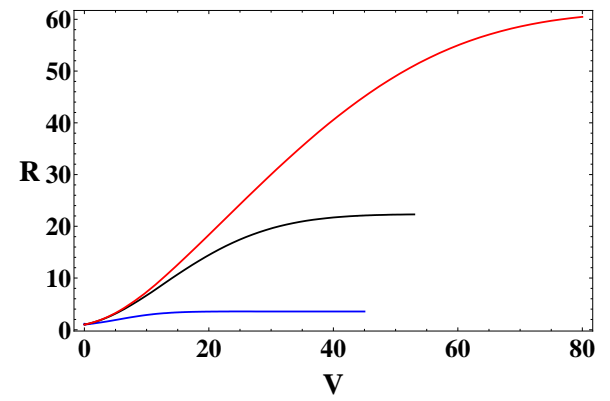

Selectivity vs. Voltage

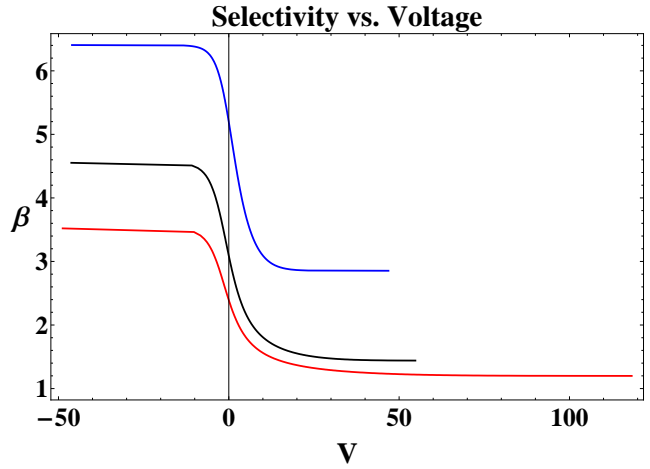

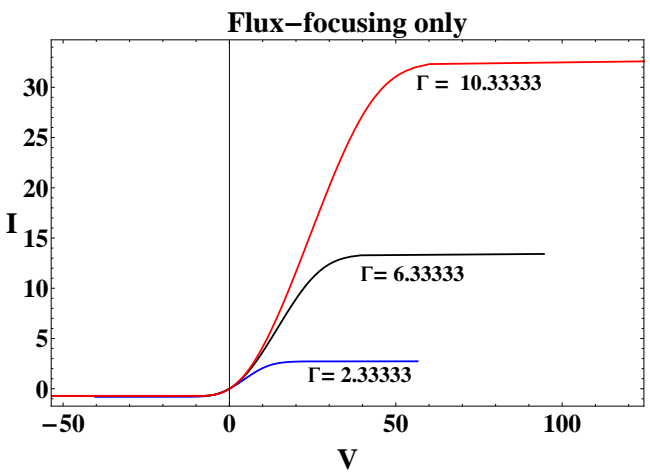

Rectification factor for flux-focusing
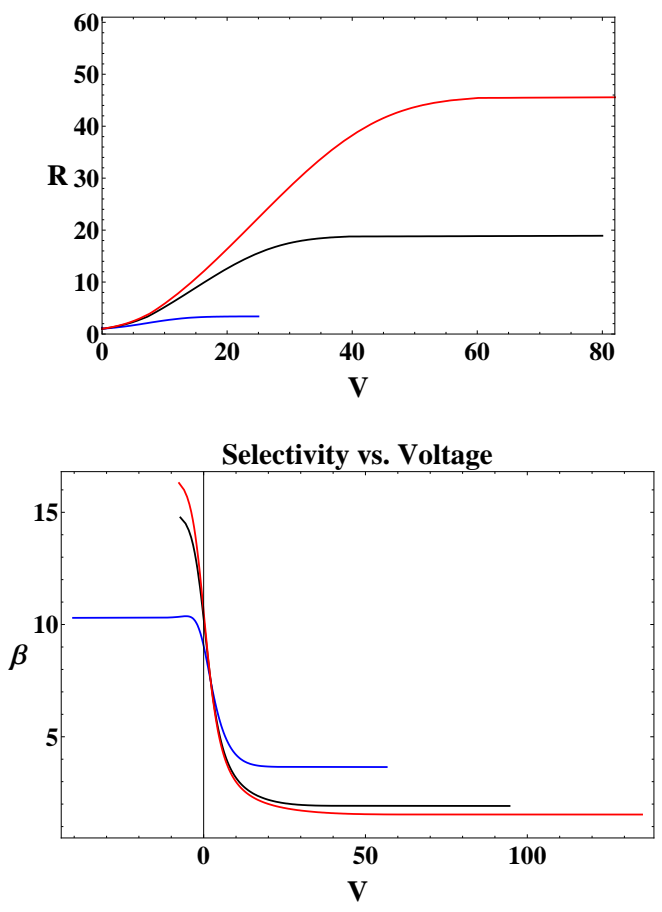

Figure 3.10: IV characteristics for non-ideal membrane with flux-focusing effect only, assuming same DL lengths, $\gamma=1$ and varying the area ratio $\Gamma$ only. All other parameters are the same as in figure 3.7 
Jarrod E. Schiffbauer Chapter 3. A classical electro-diffusive model of fluidic rectification 86 would indeed be interesting, as it is not immediately clear from the models or the equations why this should be the case.

\subsection{Summary, conclusions and future work}

The principal results of chapter 2 are summarized below, followed by a discussion of on-going and future work related to fluidic rectification.

\subsubsection{Summary and conclusions}

In the process of reviewing the body of published work on fluidic rectification and related topics, a coherent picture of the phenomenon emerges along with a general scheme for classification of fluidic rectifiers. The fundamental idea is that steady-state fluidic rectification arises because the concentration polarization layers of the device inherit the underlying device asymmetry, via asymmetric flux-densities. Thus, even in a device where the permselective element is completely symmetric, the asymmetric CPL corresponds to a voltage drop across the element which is itself asymmetric in magnitude with respect to the direction of an applied field. The work of the Siwy group [56] confirms that this effect may also be induced by applying an asymmetric concentration gradient.

Using a simple model, it is demonstrated that increasing asymmetry in the CPL, either by increasing the DL length ratio (section 2.4) or by strengthening the flux-focusing effect by increasing cross-sectional asymmetry, the strength of the rectification effect is increased as well. Because the model is a leading order model and does not include effects related to the emergence and polarization of extended space charge, it is fundamentally a low-voltage effect. However, in analyzing the low-voltage behavior, two conflicting observations are made: the voltage drop induced by the DL's upon the perm-selective element is asymmetric at all 
Jarrod E. Schiffbauer Chapter 3. A classical electro-diffusive model of fluidic rectification 87

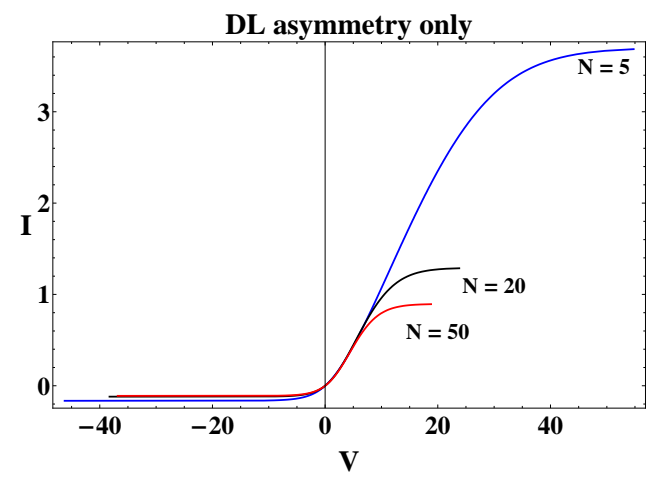

Rectification factor for DL asym.

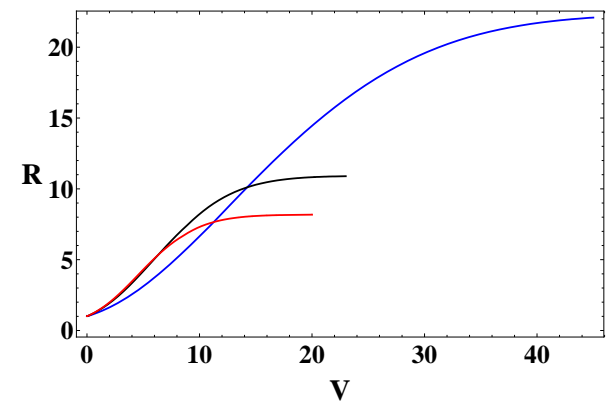

Selectivity vs. Voltage

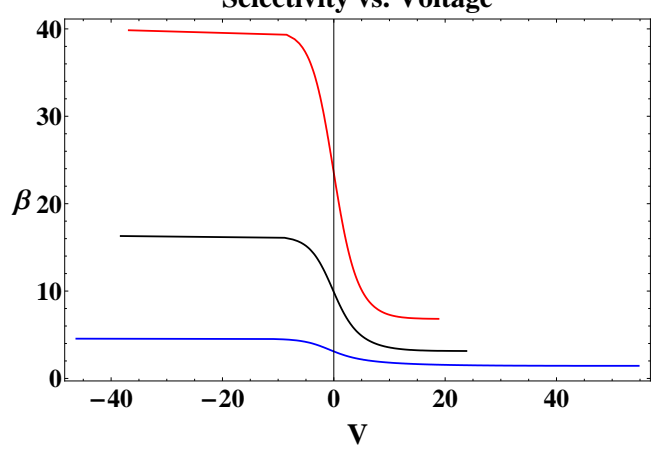

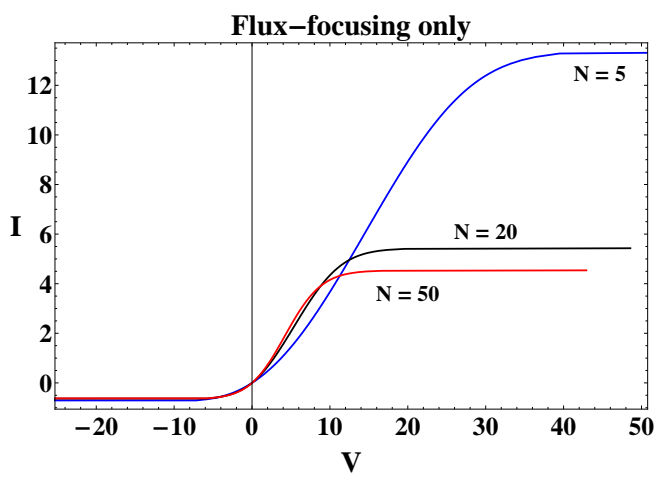

Rectification factor for flux-focusing

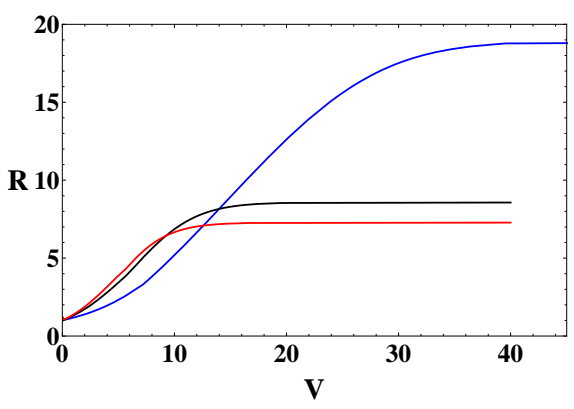

Selectivity vs. Voltage

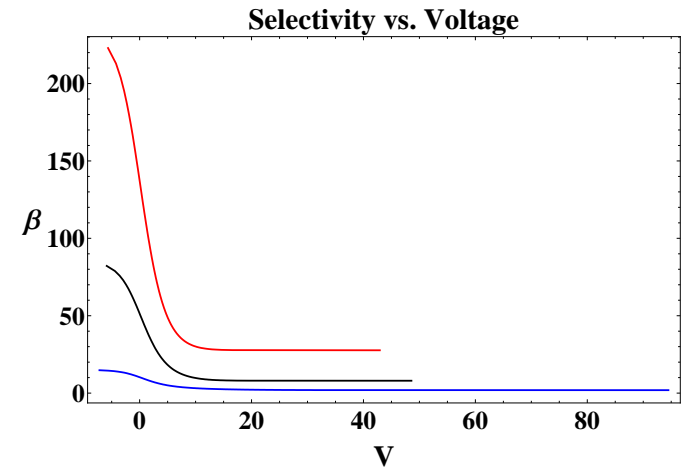

Figure 3.11: I-V characteristics and rectification factor for several $\mathrm{N}$ comparing flux-focusing to DL length asymmetry alone 
Jarrod E. Schiffbauer Chapter 3. A classical electro-diffusive model of fluidic rectification 88

currents and, at the same time a low-voltage limit for the concentration profile and membrane selectivity imply a $J_{+} \rightarrow 0$ limit for the rectification factor which is 1 to leading order. The contradiction suggests that it may be appropriate to keep the next-order terms in this limit. In any case, as will be seen in the next chapter, this leading order value seems to yield a lower-bound for experimental situations in which effects of net fluid flow and sealing issues with the membrane itself do not cause un-wanted effects.

\subsubsection{Future/continuing work}

Several questions arose during the investigation presented above. Some of these are the subject of on-going research, and others may be appropriate for future projects. In any event, while the overall picture of fluidic rectification itself is reasonably complete but broad, there are a number of un-resolved issues. Not only would further investigation of these enrich and deepen the current understanding of fluidic rectification - and aid in device optimization for practical applications - but would contribute to the fundamental understanding of electrokinetic phenomena in general. The following discussion is of course a short list, but representative of the open problems most directly related to the study of fluidic rectification.

\section{Geometry-dependent diffusion layer growth without electro-convective instabil- ity}

This is a multi-faceted issue which is the subject of several partially complete and unpublished studies within the micro-nanofluidics research group. Recent and on-going work [3] has employed finite-element methods to perform calculations in two-dimensional geometries with promising preliminary results both with and without net fluid flow. The overall goal is to better understand the relationship between fluidic geometry and the growth of boundary layers in asymmetric systems. It is suggested that the solutions to the Stephan problem [49] should be considered along with the diffusive growth models so that a better understanding of the formation of these layers, both in the classical (diffusive) regime and the super-limiting Stephan regime be explored both with and without the effects of fluid flow. As will be seen 
Jarrod E. Schiffbauer Chapter 3. A classical electro-diffusive model of fluidic rectification 89 in subsequent chapters of this thesis (and anticipated from a botdy of published work, see $[50,68,64])$ the quasi-1D electro-diffusive picture remains reasonably valid up to the point of electrokinetic instability - which can correspond to fairly high applied voltages. Competition between convective effects due to net (1D "toy") EOF should also be considered, as it may be anticipated (and shown using simple conservation arguments, as will be done subsequently) that the convective mixing length can compete with the diffusive mixing length to determine the overall extent of the so-called Nernst layers adjacent to perm-selective membranes or nanochannels which also permit fluid through-flow.

\section{Over-limiting conductance, electrokinetic instability, and DL length selection}

Well above the classical limit, it is known $[50,68,51]$ that the quasi-1D picture breaks down and the onset of more exotic electrokinetic effects, particularly related to instabilitydriven vortices, play an important role in the overlimiting current as well as selection of the effective mixing length $[53,64,66]$. However, the bulk of the theoretical work appearing in the literature concerns these effects at interfaces with ideally permselective membranes in very open geometries with no net fluid flow. As of yet, no comprehensive predictive theory of DL length selection has emerged and there are a number of confusing and (potentially) conflicting reports in the literature regarding the correlation between vortex arrays, instabilities, overlimiting conductance, and the non-linear wavenumber selection processes occurring at these interfaces. The issue itself is quite complex, and likely depends upon properties of the specific system at hand; indeed it is conceivable that there is no simple general estimate or rule.

However, application of the theory for ideal membranes presented in Refs. [50, 68] suggest that it may be possible to make some progress in this direction, and in the process, arrive at a deeper understanding of the general phenomena. The groundwork for the extension of the theories in the references to the case of non-ideal membranes and confined geometries is laid in Chapter 4. A complete study is beyond the scope of this thesis, but would make for very interesting future work. 
Jarrod E. Schiffbauer Chapter 3. A classical electro-diffusive model of fluidic rectification 90 Fluidic rectification, entropic barriers, and the relationship to kinetic models

The work of Siwy et. al. and related references [69, 44] concerning effective 1D models for the diffusion of neutral particles through passages with constrictions or bulges, i.e. 1D models with entropy barriers, suggests that such models might well describe time-dependent diffusive fluidic rectification without appealing to quasi-static diffusion layer asymmetry. Indeed, similar ideas have been employed to develop kinetic models for transport through small nanochannels in other contexts [40]. These models might offer some computational advantages over fully 2 and 3D finite-element methods, and in any case, would provide an alternative descriptive framework from which to understand the phenomena and compare results.

\section{Fluidic rectifiers and the Onsager relations}

In the 1D LEN approximation, the steady-state Navier-Stokes equation is

$$
\tilde{u}^{\prime \prime}=-\tilde{p}^{\prime}
$$

which is just

$$
\tilde{p}^{\prime}=0
$$

for an incompressible fluid. However, the total pressure may be written in terms of the mechanical and osmotic contributions [4],

$$
\tilde{p}=\tilde{p}_{m}-k T \sum_{i}^{M} n_{i}
$$

If the pressure is scaled by the osmotic value in bulk, $p_{o}=2 k T n_{o}$ for a symmetric binary electrolyte, we have

$$
p_{m}^{\prime}=-2 c^{\prime}
$$

where $c=\left(c_{+}+c_{-}\right) / 2$ as usual. Thus, the CP effect corresponds to an osmotic pressure gradient across the system. This results in a difference in mechanical pressure across the membrane,

$$
\Delta p_{m}=-2\left[c_{r}(1)+c_{\ell}(-1)\right]
$$


Jarrod E. Schiffbauer Chapter 3. A classical electro-diffusive model of fluidic rectification 91

In a symmetric system, there would be an equal and opposite osmotic contribution to the pressure gradient on either side of the membrane, regardless of the magnitude or direction of the applied electric field, so this quantity would vanish and the applied voltage will have no effect on the mechanical pressure. Thus, the only coupling between the fluid-flow and the applied voltage is due to the electric body force in the boundary layers (which, in the LEN approximation we are assuming to be vanishingly small in the micro-system) and the coupling coefficients are known to obey Onsager relations [36, 4]. In an asymmetric system, the drop in mechanical pressure across the membrane reflects the asymmetry in the CPL's and the magnitude of the associated pressure drop will now depend on both the magnitude of the voltage and the direction of the field. This should correspond to an induced fluid flow across the membrane and it seems natural to wonder how this modifies the relationship between coupling coefficients. Study of the problem from the suggested point of view of the previous subsection, i.e. Fick-Jacobs and Fokker-Planck-like formulations, would be an interesting compliment and might help to cast the problem in terms of the original arguments of Onsager, i.e. in terms of reversibility. 


\section{Chapter 4}

\section{Analysis of experimental data}

\subsection{Chapter introduction and overview}

Chapter 4 concerns the analysis of a variety of experimental results using the NCM/NMI device and a similar device with a Nafion membrane. An overview of experimental details is given in section 4.2. Section 4.3 contains an analysis of previously published data [37] comparing the Nafion and NCM devices in different device configurations while Second 3.4 presents an analysis of new data, including the effect of varying asymmetry and bulk concentration on I-V curves and rectification strength. The end of section 3.4 contains an analysis of a "chemical ratchet effect", which is shown to be fundamentally related to the rectification phenomenon. Section 3.5 contains an analysis of the early stages of sample concentration in the NCM/NMI under very high reverse bias.

The theory developed in chapter 3 may be used to qualitatively understand the results of sections 4.3 and 4.4. However, several factors, including the comparably large operating voltages and difficulties with reproducibility make it difficult to verify any quantitative predictions. Furthermore, some of the observed trends provide a conflicting picture, which may have to do with a number of effects not included in the models of chapter 3 . Despite these difficulties, the theory is able to account for several important qualitative behaviors in the rectifiers, and it provides a simple explanation of the hysteresis effect. Furthermore, the comparison of the NCM and Nafion systems reveals an interesting persistent feature 
in the I-t behavior not previously reported which seems nonetheless intimately related to the breakdown of quasi-1D behavior and a change in the stability of the system. Also, the high-voltage rectification effect, seen both in these devices and those in Ref [65], is explained here as a secondary - though very strong- effect due to the suppression of electro-convective mixing by the microchannel walls in the off-state. The analysis of the concentration process reveals complex dynamics, with very different behaviors at different times, including evidence of electro-convective instabilities quite unlike those related to the vortices caused by non-equilibrium electro-osmotic slip. Finally, in section 4.5 a number of additional experiments are suggested which might help to test the applicability of the theory of chapter 3 and clarify some of the difficulties with these systems.

\subsection{Overview of experimental details}

\subsubsection{A brief description of the experimental set-up and process}

Figure 4.1 shows the two device configurations used to obtain the experimental data. Both devices consist of a $2 \mathrm{~cm}$ long microchannel etched into a glass slide using standard photolithography techniques. The channels are roughly trapezoidal in cross-section with a mean width on the order of $\approx 200 \mu \mathrm{m}$ and depths varying from approximately 12 to $40 \mu \mathrm{m}$ a in the side-mounted device. The horizontal connecting channel in the top-mounted device is approximately $12 \mu \mathrm{m}$ a deep, however the relevant cross-section is that of the $150 \mu \mathrm{m}$-diameter vertical access to the membrane interface. In both cases, the reservoir-entrance is approximately $1 \mathrm{~mm}$ in diameter, with an O-ring of i.d. $0.8 \mathrm{~mm}$ seating the membrane against the chip (O-ring not shown.)

The reservoir entrances are approximately cylindrical and made of Delrin and/or Teflon, in the side-mounted configuration (thus unlikely to generate much EOF.) The longer entrance for the side-mounted configuration is a somewhat irregular cylinder. In all experiments, a 
(a)

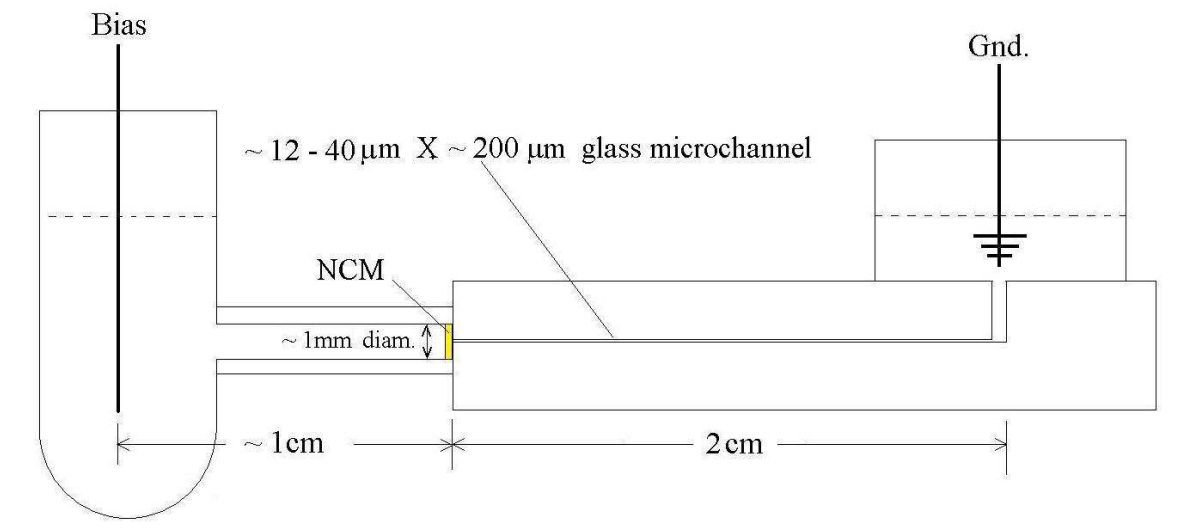

(b)

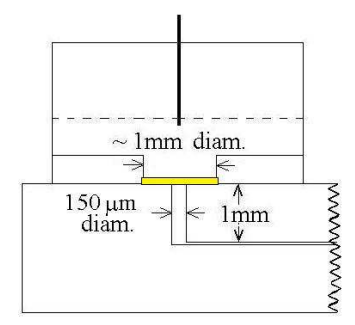

Figure 4.1: Diagram showing geometries and approximate dimensions for the two device configurations used in the experiments, (a) the side-mounted membrane device and (b) the top-mounted membrane device 


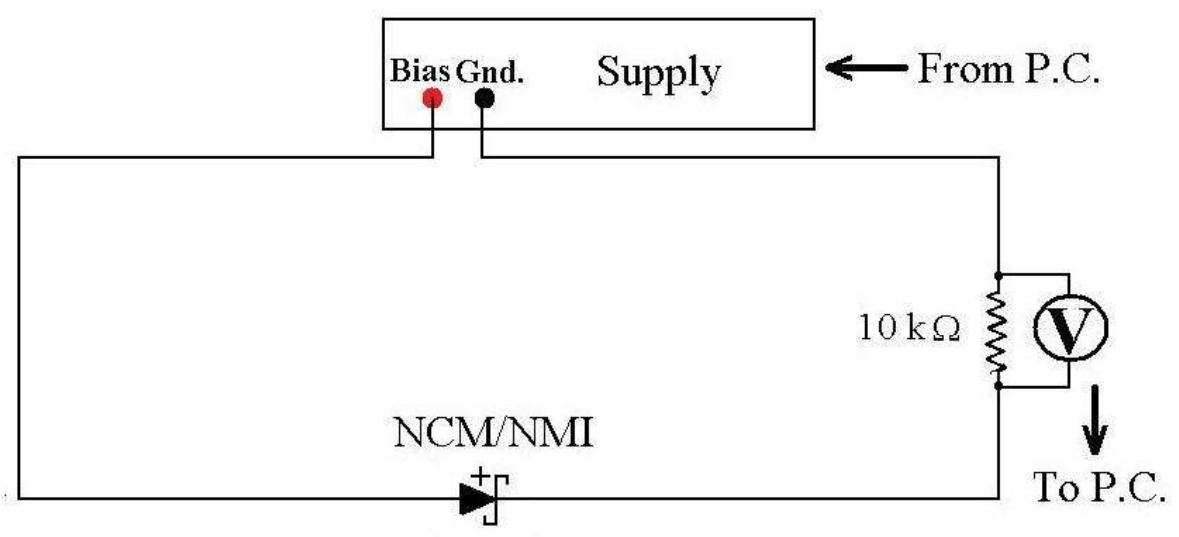

Figure 4.2: Schematic of experimental set-up for data collection. Note: NMI fluidic rectifier represented by a the symbol for a Schottky diode with the ' + ' denoting cation-selectivity.

sodium phosphate buffer at $\mathrm{pH} 7$ is used at three different concentrations, $1 \mathrm{mM}, 10 \mathrm{mM}$, and $20 \mathrm{mM}$. Note, this is not a binary buffer, however since only qualitative comparisons to theory are being made, this is not a critical issue. The reservoirs are filled so the fill-lines are level (see Figure 4.1.) Devices for the LOC paper were allowed to sit filled for an hour before measurements, those used in the newer experiments were de-gassed in a vacuum chamber for a minimum of one hour, and a maximum determined by the patience of the particular experimenter and/or the apparent cessation of bubble formation. For some of the newer data, the $\mathrm{NaOH}$ pre-rinse was omitted, resulting in some additional noise in the data but no other readily apparent differences. It was hoped that if the glass microchannel surface were not actively de-protonated, the net device EOF might be lower if this resulted in less dissociation of the Silanol groups in the glass microchannel. Omission of this step proved to be neither particularly useful nor detrimental.

The fluidic rectifier is connected to a computer-controlled high-voltage power-supply via two platinum wire electrodes, with the current through the system determined from the voltage drop in the ground-line resistor, as shown in Figure 4.2. The voltage drop is measured 
with a standard digital multi-meter and data collected at a rate of 1 measurement per second. This reduces the drop across the rectifier by a small amount compared to the full applied voltage, however for measurements at the present ranges, this is negligible (i.e. typically no more than a few to a few tens of millivolts.) The voltage-stepping of the high-voltage power supply is controlled by a Labwindows program, and the exact sequences employed varied somewhat, covering three ranges: $-800 \mathrm{~V}$ to $800 \mathrm{~V}$ for the Nafion and PC-NCM comparisons in the LOC paper, $-80 \mathrm{~V}$ to $80 \mathrm{~V}$ for experiments using a side-mounted PC-NCM in devices with a range of microchannel sizes, and $-20 \mathrm{~V}$ to $20 \mathrm{~V}$ for side-mounted PC-NCM on a 40 micron channel at three different buffer strengths. In all cases, the voltages were swept at a rate of 5 minutes per voltage.

The equipment, set-up, and procedures for the experiments in the references and the new experiments presented in this chapter are similar to those used in the cited publications. Relevant details for the current-voltage characteristic experiments are discussed in the sub-section below. Some details regarding the concentration and band-broadening experiments are given in section 4.4. For further information on these details, error bars, etc., the interested reader is referred to $[37,24]$

\subsubsection{A typical I-t curve for the NCM/NMI}

While I-t characteristics for different device configurations and parameters as well as individual runs on the same device tended to vary somewhat, several features common to some degree to all of the runs can be seen in Figure 4.3. This is even true with the same membrane under ostensibly the same experimental conditions. However at least part of this issue has to do with the so-called chemical ratchet effect discussed later on in this chapter. Another problem with reproducibility has to do with ensuring a good membrane seal without creasing the membrane or breaking off the reservoir housing. This appears to be more of a problem with the PC-NCM in the side-mounted configuration, while not apparently affecting the Nafion device. This may be in large part due to the fact that the Nafion effectively suppresses net hydrodynamic flow while not only do NCM's permit some flow, they may also 


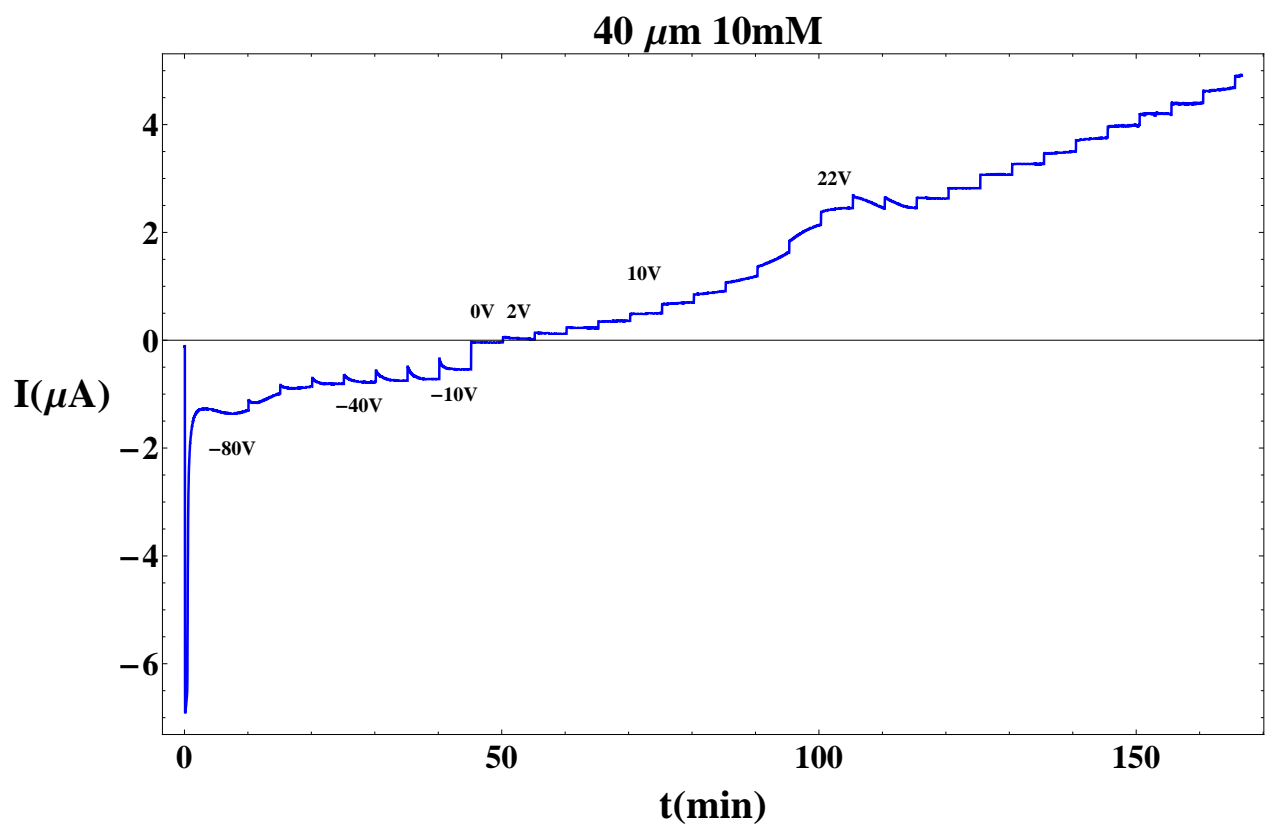

Figure 4.3: I-t response for a 40 micron channel with $10 \mathrm{mM}$ buffer with stepped voltage applied showing several typical features for the NCM/NMI. Note the voltage intervals are not all equal; $10 \mathrm{~V}$ steps are used in the off-state and $2 \mathrm{~V}$ steps in the on-state.

generate significant amounts [27] of it.

The low-voltage behavior can be characterized by a very nearly quasi-steady current, which, according to the ideas discussed in chapter 1, is consistent with expectations for a device with a perm-selective interface operating below the classical limiting current. In some cases, a slight, monotonically decreasing transient is present at the first positive voltage in a sequence. But this feature is not ubiquitous and may have only occurred in systems which were not allowed to rest sufficiently between off- and on- state voltages so as to dissipate residual concentration gradients. In any case, the appearance of a persistently increasing slope, i.e. such that the magnitude of the current increases with time while held at a given voltage, can 
be taken as an indication of the appearance of an extended space charge and possibly the onset of electro-convection below voltages high enough to sustain fully-formed vortices. Full development of electro-convection does not seem to occur immediately above some specific voltage, nor does it follow the same course of events in the NCM device as does a Nafion device. While these considerations prevent the establishment of a well-defined experimentally observable criterion for the critical voltage corresponding to the classical limiting current corresponding to the knees of Chapter 2, they provide some guidance in comparing the onset of effects precipitated by the breakdown of local electro-neutrality above the limiting current. In particular, there is an inflection in the NCM I-t slope (at about $22 \mathrm{~V}$ in the example figure) occurring at some point above the threshold for the formation of extended space charge, and likely related to a change in electro-hydrodynamic stability. To the author's knowledge, this feature has not been reported before. ${ }^{1}$ The LEN theory might reasonably be expected to yield some insight into the behavior of these devices below -and perhaps quite close to- this point, but cannot be expected to have any quantitative predictive power anywhere except perhaps the lowest voltages. However, given the estimates presented in the following chapter and the apparent systematic drift of about $2 \mathrm{~V}$ of the power supply, it is unlikely that the current experimental set-up would be useful for such low-voltage $(<0.5 \mathrm{~V})$ measurements.

One important qualitative note is the distinct differences between the appearance of the transient current in the off- and on- states. If, as seems reasonable, the appearance of extended space charge and the onset of electro-convection can be associated with the transient behavior in the system, it appears that (at least for the NCM devices) the limiting current is reached at much lower voltages in the off- state as compared to the on-state. This is consistent with the predictions of the simple theory in the previous chapter; asymmetric devices will have lower off-state limiting currents corresponding to the appearance of extended space charge at lower voltages. But note, unlike the on-state (particularly the on-state for the Nafion device, as will be seen subsequently) the off-state current appears to rapidly reach a quasi-steady value. This does not necessarily correspond to a complete absence of electroconvective vortices, however, as will be seen; their behavior in these systems is somewhat different than in other systems, in particular, those observed in the Yossifon and Chang

\footnotetext{
${ }^{1}$ Is it possible these are somehow related to Teorell oscillations? See for instance, chapter 5 of $[46, ?]$
} 
experiments $[64,5,66]$.

As stated above, the macroscopic LEN violation which can lead to Rubinstein's electroosmotic instability may be associated with the appearance of a net increasing slope in the I-t curves. This may be in part due to the importance of the intermediate time-scales at currents above the classical limit $[52,1]$. This may be most apparent in the intermediatevoltage time-dependence of the off-state system. In the on-state, a small slope begins to emerge at intermediate voltages, which may correspond to the emergence of an extended SCL and the onset of electro-osmotic convection cells of the type predicted by Rubinstein and Zaltzman's work $[49,50,68]$. In general, these effects seem to appear at voltages several orders of magnitude above the theoretically estimated threshold. Even the lower voltages (10V) corresponding to the onset of electro-osmotically driven vortices in other nano-channel systems, notably those of Yossifon and Chang [66, 5, 64], are considerably higher than the estimates, leaving a very wide voltage window between predicted ESC development and the onset of electro-convection. This may be attributable to net EOF and/or to the fact that the nanochannel/nanopore systems allow significant co-ion fluxes through the perm-selective element in comparison to the ideal models used to develop the theory. The issue of extending the theory of non-equilibrium electro-osmotic slip used in the determination of the associated stability criteria is discussed extensively in the next chapter, along with some preliminary work in that direction.

A second feature, at a higher voltage than the appearance of the non-zero (increasing) I-t slope is the inflection of the slope at a critical voltage. What is not clear is whether or not this corresponds to the full development of (stable) vortices according to the Rubsinstein and Zaltzman model (with appropriately modified slip conditions) or if this is some other process. A different type of instability, known to be responsible for mixing in other electrokinetic systems, that of an electro-viscous instability ([6].) Also note that sharp transients in the off-states tend to appear at much lower magnitudes of negative voltage than transients in on-states, indicating that non-locally electro-neutral effects are observable which is in some sense, consistent with expectations based on the 1D LEN theory. 


\subsection{Fluidic rectification in two types of asymmetric PC-NCM and Nafion membrane device} 2

\subsubsection{Top-mounted PC-NCM device}

The I-t response for a typical run of the top-mounted NCM device is shown in Figure 4.4. Several similar runs were collected and time-averaged over each voltage step to produce I-V curves. The average I-V curve is shown in Figure 4.5, further information on the averaging and statistics may be found in [37]. The low-voltage behavior is typical and nearly linear at very low voltages. However, for this particular system, 'very low' seems to mean voltages in the range $\pm 100 \mathrm{~V}$, which almost 4 orders of magnitude higher than the most reasonable estimate for the critical voltage corresponding to the classical limiting current (see chapter 5, Table 3.1.) Even at these low voltages, it is apparent that the the device exhibits noticeable rectification. It is difficult to assess to what degree these effects can attributed to polarization of extended space charge. However, because of the device design, for modest space charge layers up to several microns, it is unlikely that asymmetry of the space charge alone can account for the effect.

At or around $150 \mathrm{~V}$, it is apparent that the I-t slope increases markedly over the 5 minute measurement interval. The I-t inflection occurs between $300 \mathrm{~V}$ and $400 \mathrm{~V}$, more than an order of magnitude higher than what is seen in the side-mounted NCM devices. After this point, the current-voltage relationship appears to be approximately linear again with a higher overlimiting conductance, similar to that observed in other systems [67, 64], albeit this occurs at much higher voltages in this particular system. Given the nature of this system, i.e. a membrane with 10nm diameter pores which are comparatively widely spaced, the limiting current plateau should not be discernable [67] and the apparent plateau in the I-V curve (Figure 4.5) is related to the inflection. What is not clear is the connection between the inflection, Rubinstein and Zaltzman's instability, and the vortices which are anticipated to

\footnotetext{
${ }^{2}$ The data analyzed in this section was originally published in $[37,24]$
} 


\section{Top-mounted NCM/NMI I vs. t for stepped voltage}

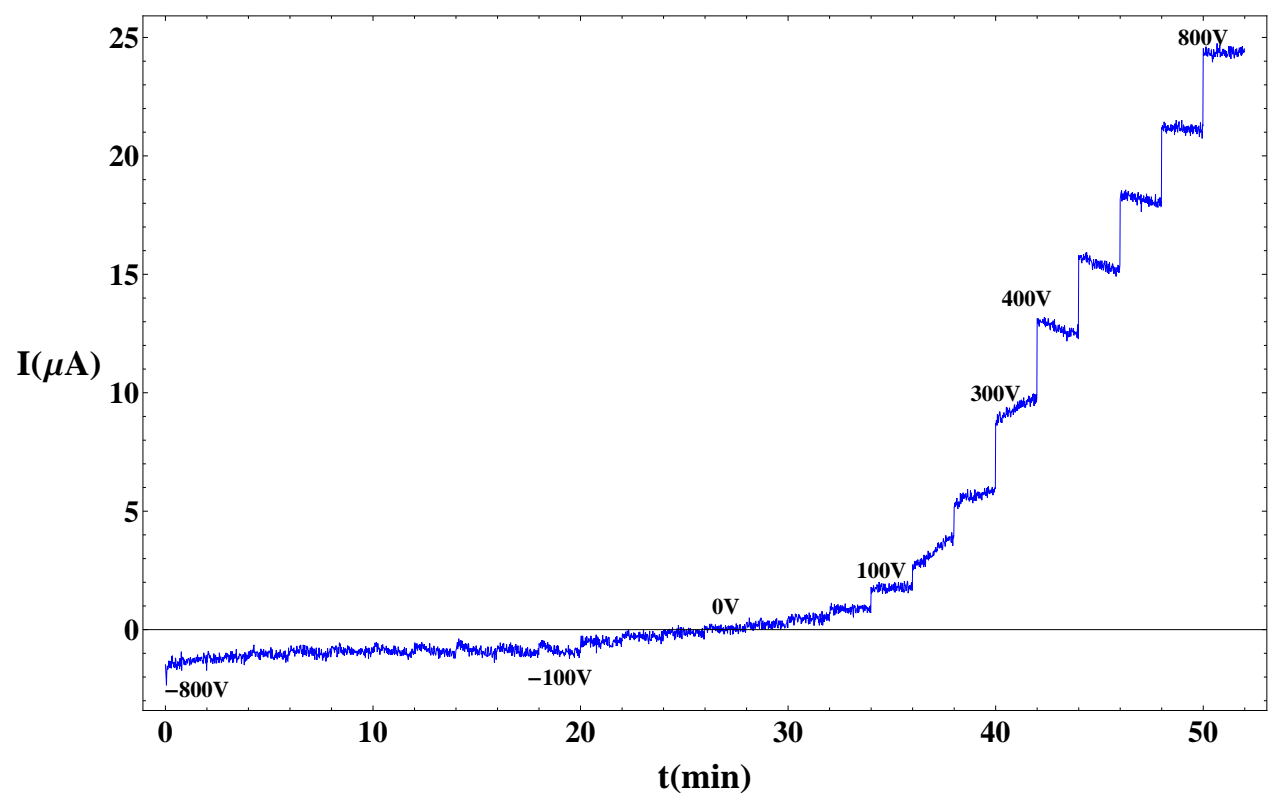

Figure 4.4: I-t curve for a top-mounted PC-NCM/NMI showing several distinct response regimes. In particular, note the differences between the regions $-400 \mathrm{~V}$ to $-100 \mathrm{~V}$ and between $100 \mathrm{~V}$ - and $400 \mathrm{~V}$.

develop because of Dukhin's EOF of the second kind.

The off-state NCM device seems to have three distinct regimes of behavior: low-voltage between $0 \mathrm{~V}$ and about -100V, intermediate between about $-100 \mathrm{~V}$ and $-400 \mathrm{~V}$, and high off-state voltages, between about $-400 \mathrm{~V}$ and $-800 \mathrm{~V}$. The lowest off-state I-V response is nearly linear. For voltages greater in magnitude than about -100V, the off-state exhibits the characteristic, relatively-rapid current transient, which as mentioned, likely corresponds to super-critical electro-convective effects in the microchannel. However, unlike the corresponding effects in the reservoir, it is believed that confinement in the channel prevents mixing with bulk-like electrolyte, hence the current becomes limited by the confining geometry of the microchan- 


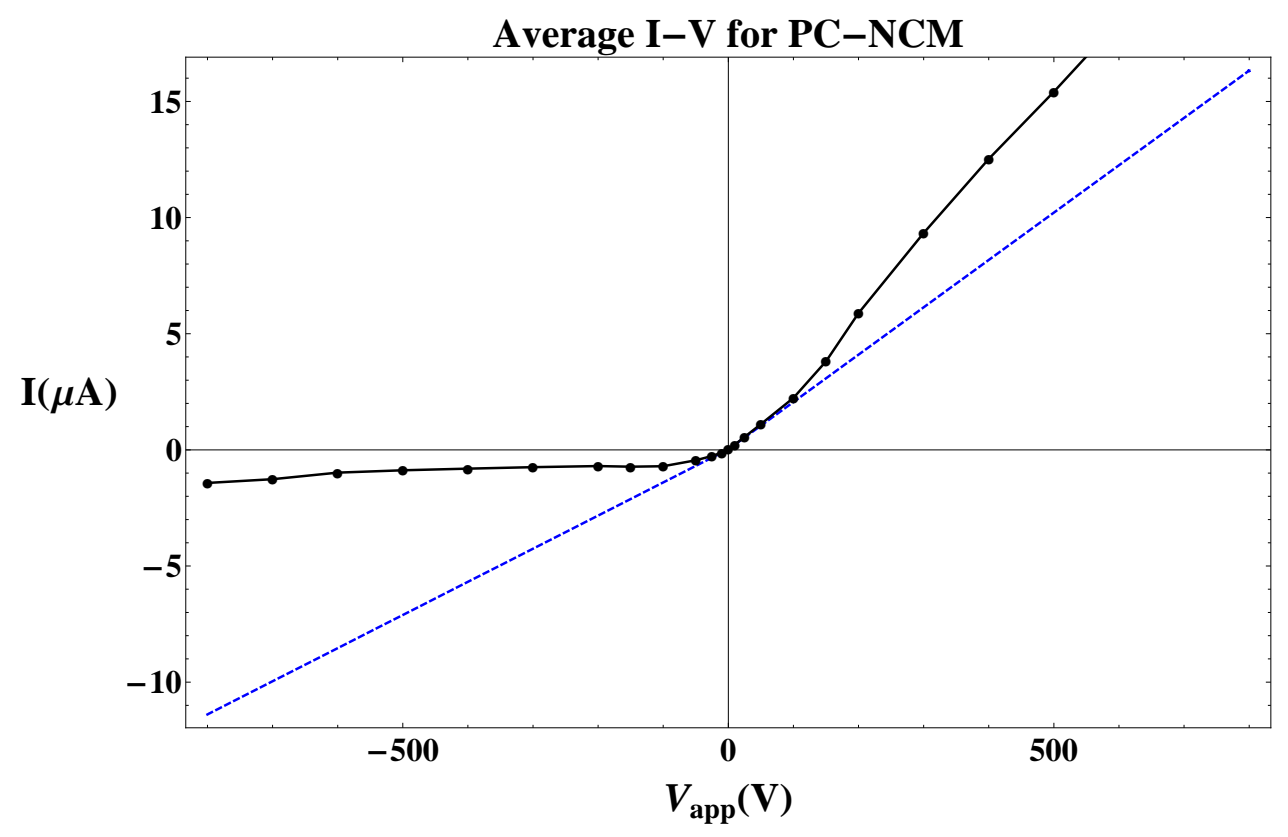

Figure 4.5: Interpolated I-V curve for top-mounted NCM device (solid line) with averaged $\mathrm{I}-\mathrm{V}$ data points. Dashed lines are linear extrapolations of the I-V behavior on either side of the origin, the slopes of which are almost indistinguishable by inspection.

nel itself in the off-state. At voltages much below $-400 \mathrm{~V}$, this well-defined transient current increase is no longer as apparent in the I-t curves. This may be because the threshold for the Q1DL layer which supports the formation of these vortices has been exceeded (see Ref. [68] and Table 3.1 in chapter 5.) Some imaging and analysis of vortex dynamics has been done for the NCM/NMI system which helps to shed some light on the behavior at very high negative voltages, as will be discussed later in this chapter. In particular, there is an absence of any stable vortex pattern. However, as will be shown, there is evidence of transient vortex formation and annihilation. It would be interesting to analyze images from lower magnitude (i.e. up to about -100 to - 200V) off-state voltages as well to determine whether or not and how vortices form and/or propagate at lower voltages. 
Figure 4.5 shows an ensemble average over 5 runs of the time-averaged I-V characteristics. The dashed lines show the extrapolated linear behavior, and the slopes are (very slightly in this case) different on each side of the origin. There is some ambiguity since the lowest voltage magnitude is $10 \mathrm{~V}$ here, so the actual response at very low voltages can only be estimated. Note the on-state response remains very close to linear as high as 100V. This is consistent with the observation that significant I-t transience only occurs at higher voltages. The rectification factor vs. voltage for the top-mounted NCM is shown in Figure 4.6, and is defined as the absolute value of the ratio of on- to off- state current as a function of voltage.

The rectification factor is calculated in two ways. Since many of the runs do not have matched pairs of off-on voltages, i.e. data points at every $\pm V_{a p p}$, a polynomial interpolation of the I-V data was carried out. The optimal order of the interpolation was determined by comparing the interpolated I-V curve to the original I-V data points. For the high-voltage devices, where there were more matched data pairs, a fourth-order interpolation produced the best curves. For the matched pairs, the rectification factor was calculated directly. Both are plotted in the figure, the points showing the pairs and the solid line is a plot of the rectification factor as calculated from the interpolated I-V curves. The dashed line shows a limiting value obtained by taking a ratio of the linear I-V extrapolations (dashed lines in Figure 4.5.) Hence, both the interpolated rectification factor and this extrapolated limit blow up as $V \rightarrow 0$. These low-voltage blow-ups can be ignored as artifacts of the interpolation process. As was noted in chapter 2, the classical electro-diffusive formulation has a limiting value of $R=1$ at $V=0$, and the value from both the interpolated and extrapolated data appears to bottom out at approximately twice this value.

Qualitatively, this curve is very similar to the published rectification factor curves for the Yossifon-Chang rectifier [65], and it is evident that there is significant low-voltage rectification. The much larger rectification factor at super-critical voltages occurs because mixing is inhibited in the microchannel off-state while it can occur freely in the reservoir on-state. Thus there is a secondary rectification effect due entirely to the suppression of over-limiting 


\section{Rectification factor for PC-NCM}

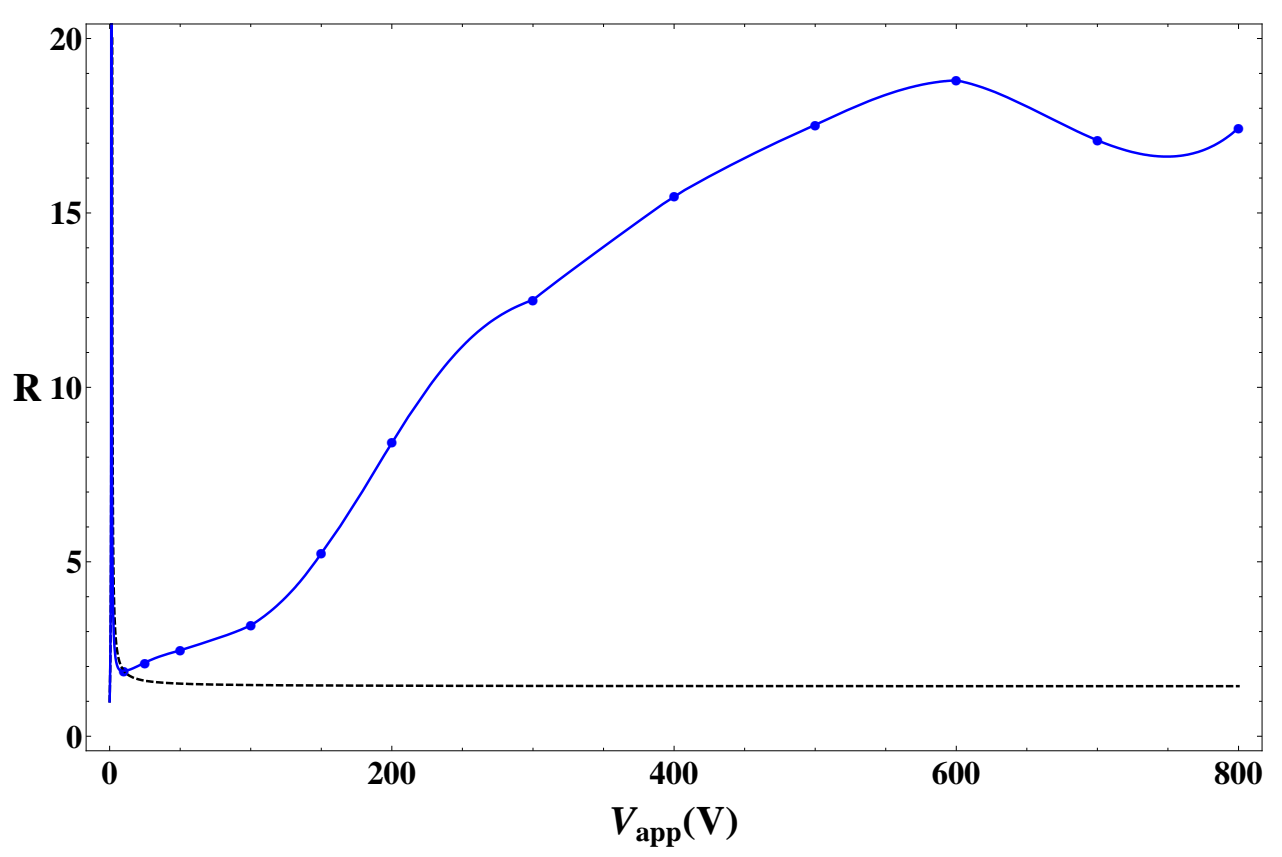

Figure 4.6: Rectification factor for top-mounted NCM device, interpolated (solid line), data points (points,) and extrapolated experimental $V=0$ limit (dashed line.) 
conductance by the microchannel. This effect is extremely strong and allows the device to pass very high currents in the on-state, which makes the on-state device advantageous as a sample concentrator, since (as shown in both chs. 2 and 3) the (LEN) on-state concentration profile increases linearly with the current. The 'classical' electrodiffusive rectification behavior is evident up to about $100 \mathrm{~V}$, as anticipated from the I-t and I-V curves, is qualitatively similar to the curves in chapter 2 up to this point, and also of the same order of magnitude.

\subsubsection{Side-mounted Nafion device}

By comparison, the I-t behavior of the Nafion device is much cleaner; there is very little noise evident in these plots and one possible reason is that net EOF is largely responsible for the noise, and Nafion suppresses the net fluid flow. In general, the magnitudes of the currents are too small to determine what is happening in the off-state, but apparently several features evident in the PC-NCM devices are absent, or at least appear markedly different in the side-mounted Nafion device. Another possible reason is that the Duhkin's EOF of the 2nd kind vortices appear at very low voltages in the PC-NCM, causing excess electrical noise, which is not evident in the Nafion device. There are a number of other interesting points. There is little to no evidence of the off-state transient behavior seen in the top-mounted PCNCM device, which suggests that net EOF may play some role in this phenomenon. Note also that, even at the highest on-state voltages, where the EC vortices are expected to act, the signal is relatively clean. Even though the cross-sectional asymmetry is greater for the side-mounted configuration than the top-mounted, the apparent limiting current and LEN threshold voltage are considerably lower, occurring somewhere between $50 \mathrm{~V}$ and $100 \mathrm{~V}$ in the on-state. However, since it is known that the fixed charge density in Nafion is higher (and the effective diffusivity lower,) this is not necessarily inconsistent with the LEN predictions. To make the comparison complete, it would be necessary to study a top-mounted Nafion device, and neither this data nor the device were available.

In addition to the lower threshold voltage (and presumably lower classical limiting current,) the over-limiting behavior of the Nafion device is noticeably different. There is no 
Side-mounted Nafion I vs. $t$ for stepped voltage

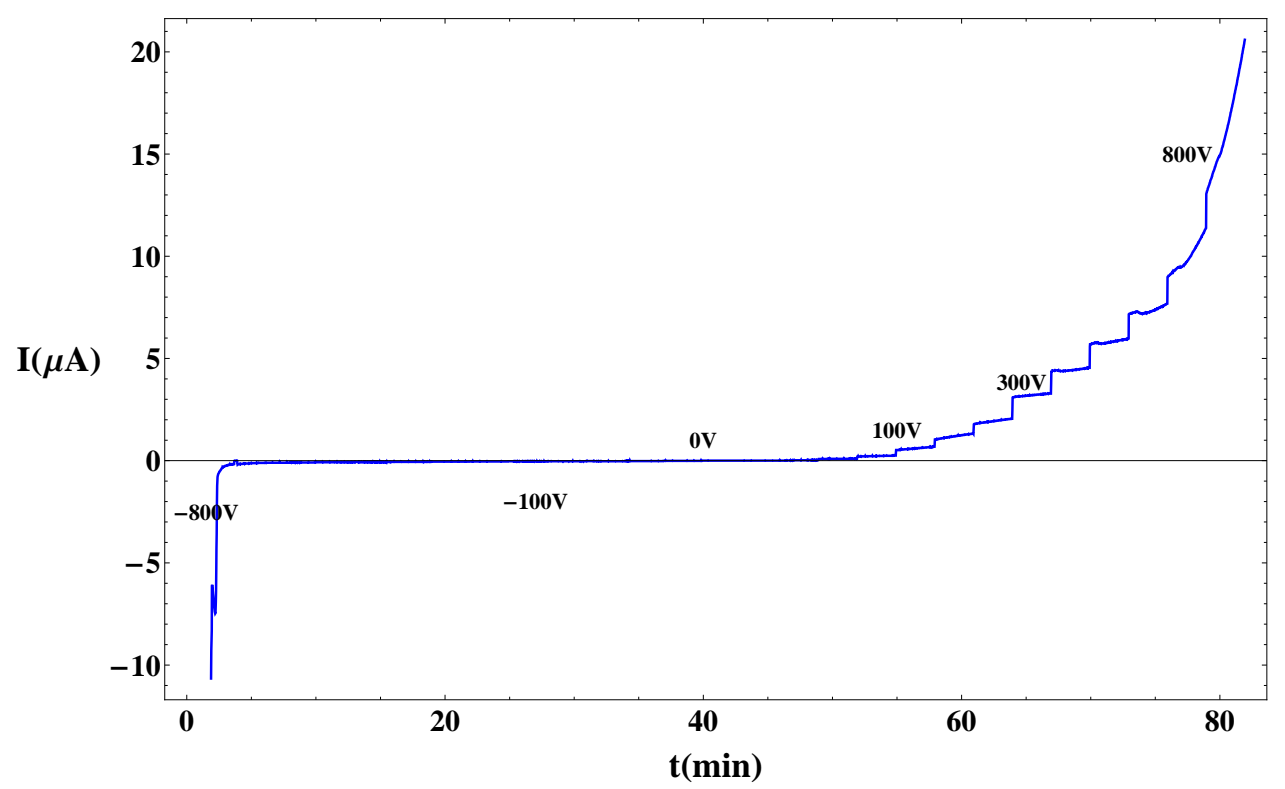

Figure 4.7: I-t curve for a side-mounted Nafion device. Note the relatively steady currents (albeit with barely perceptible slope at voltages as low as $100 \mathrm{~V}$ ) up to voltages close to $300 \mathrm{~V}$ and the marked increase between $600 \mathrm{~V}$ and $700 \mathrm{~V}$. The off-state transients appear to be suppressed.

apparent inflection, and the I-t slope just appears to increase indefinitely with increasing voltage. Note this implies a much higher over-limiting conductance, relative to the sublimiting behavior, in comparison to the NCM device. Since the I-t inflection in the on-state appears in both side-mounted and top-mounted NCM devices, we can safely conclude that the difference has to do with the membranes themselves and net EOF is the likely culprit. Another way to test this would be to use coated microchannels that suppress EOF and see if this limits the I-t noise and significantly changes the I-t inflection and off-state transients. However, given that NCM's can produce significant EOF due to the relatively large electric body force in the overlapped double-layers, this may not be a viable approach. 


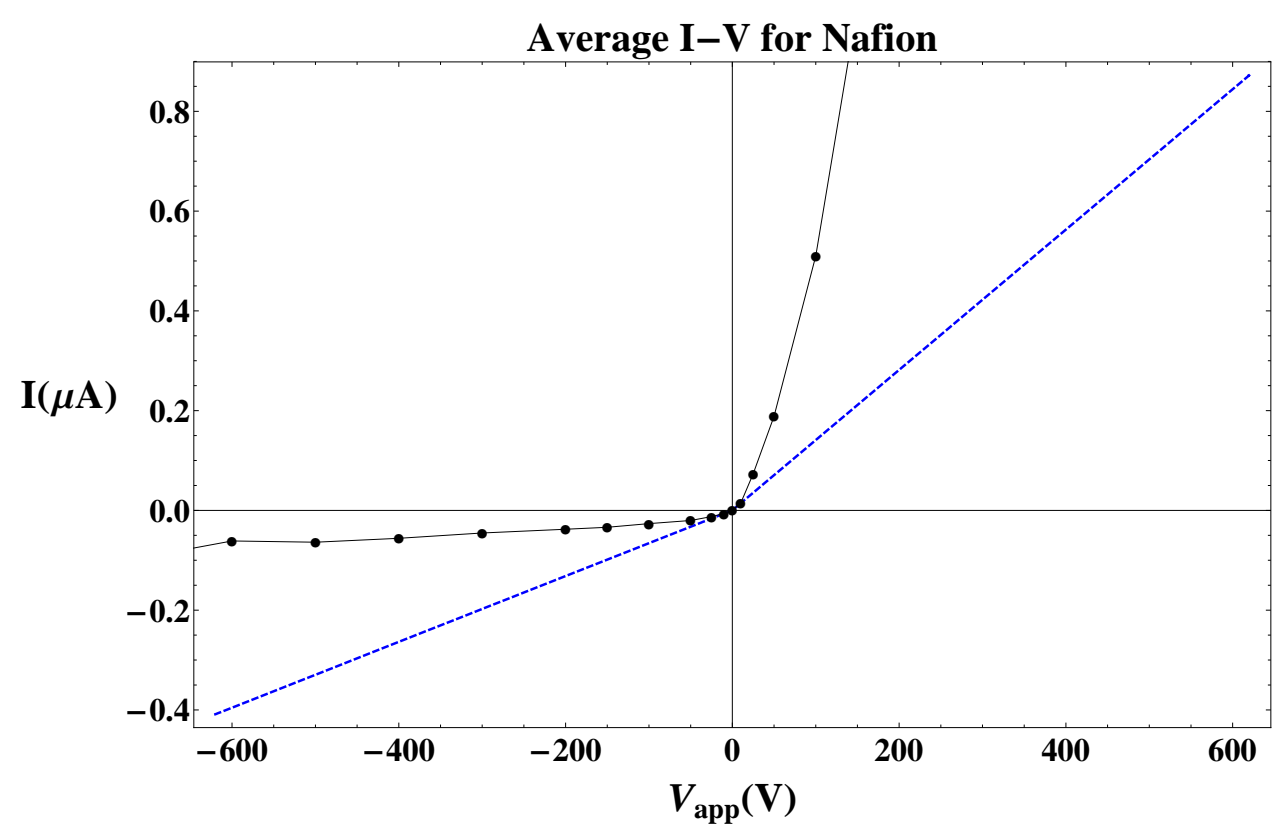

Figure 4.8: Time-averaged and interpolated I-V curve for a side-mounted Nafion device (solid line), time-averaged I-V data points, and extrapolated $V=0$ lines.

Overall, the Nafion device is a stronger rectifier, despite the fact that the fixed charge density is expected to be much higher than that of the PC-NCM. This may be understood -at least at sub-critical voltages- by considering that, according to the models of chapter 3 , changing the device asymmetry has a more profound effect on the I-V response and rectification factor than does changing the charge density. However, this has little bearing on the difference in performance at very high voltages and it must be concluded that electro-convective effects near NCM's are markedly different than Nafion membranes. Again, the likely culprit is net $\mathrm{EOF}$, however the question of stability for non-ideal membranes is open and may be relevant in this case. The NCM is much more non-ideal than the Nafion membrane, even ignoring net fluid flow. Both net fluid flow and passage of a significant number of co-ions can be anticipated to affect the electro-hydrodynamic stability of the system. 


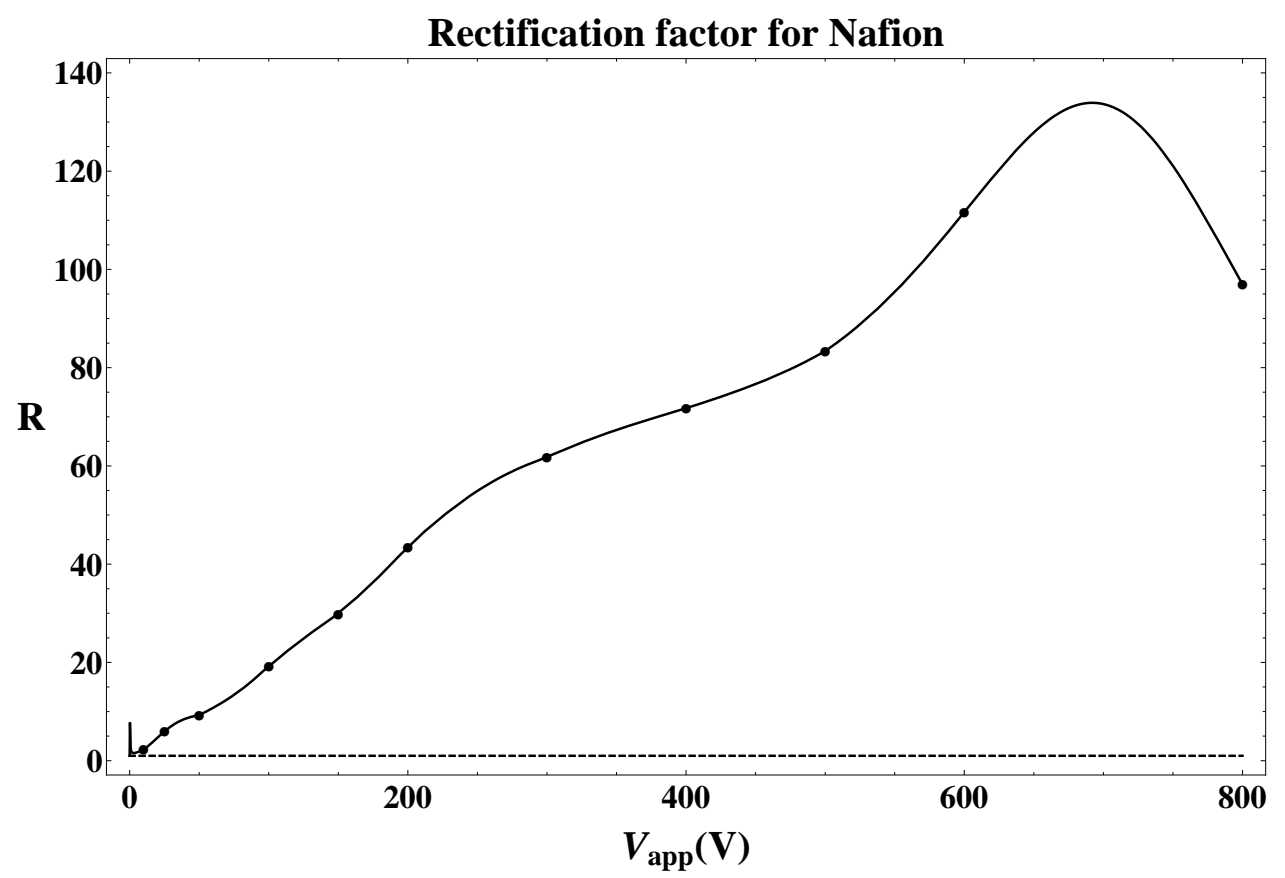

Figure 4.9: Rectification factor for side-mounted Nafion device, interpolation (solid line,) data points (points,) and the classical (theoretical) low-voltage limit, $R=1$, (dashed.) Note the Nafion device approaches this limit much more convincingly than the NCM, which may indicate the dominance of electro-diffusive transport in the former and the importance of EOF in the latter

\subsubsection{Comparison of the current density and low-voltage rectifi- cation}

It is somewhat misleading to compare the total current in these devices, and in fact, since it is the current density at the membrane-electrolyte interface that determines whether or not the system is being driven above the classical limit, it is desirable to look at the current density, normalized by the cross-section of the microchannel (since this is approximately the 


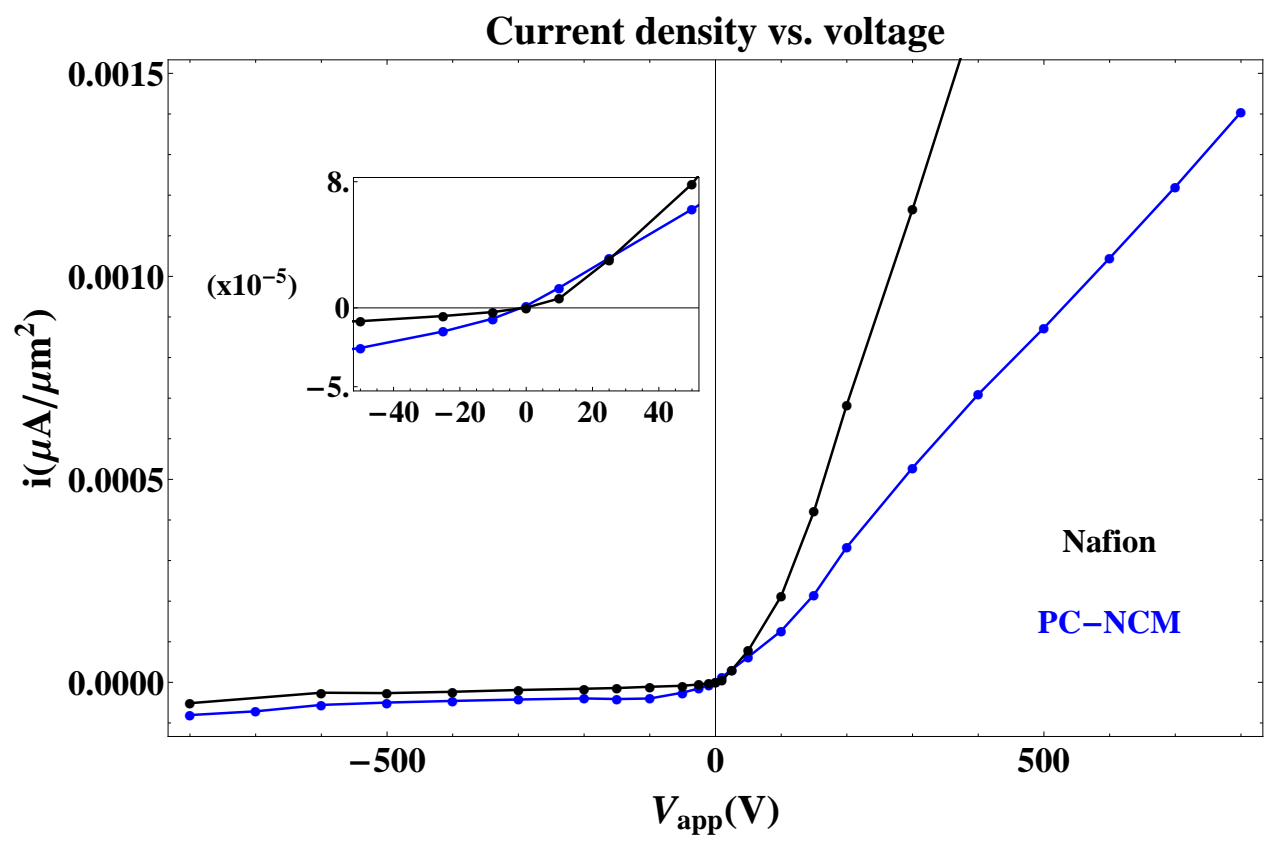

Figure 4.10: Comparison of current density vs. voltage, w/ current normalized by channel cross-section. Inset shows very low-voltage behavior, though given the sparsity of data points, that the Nafion current density appears to be lower than the NCM current density at very low voltages must be taken with a grain of salt.

same 'active' surface area on both sides of the membrane.) Upon doing so, as in Figure 4.10, several things become clearer. First, the sub-limiting behavior of the systems is comparable and consistent with expectations derived from the LEN theory. The current density in the off-state Nafion device is lower; it has a higher charge density (and no net EOF.) The over-limiting current density in the Nafion device is much higher than either the sub-limiting current density, or the NCM current density. For the NCM, the conductance of the system does not radically depart from the value near the critical voltage, but in fact, above the I-t inflection point, seems to return to a value rather close to the sub-limiting conductance, which appears to confirm the picture provided by the elimination of the limiting current plateau via the field focusing effect [67]. 
A comparison of the low-voltage rectification factors is given in Figure 4.11 along with the low-voltage limit $R=1$ from Chapter 2. Again, the Nafion is obviously a much stronger rectifier, even at low voltages. Here it is tempting to attribute this to a combination of device asymmetry and the effects of net EOF. As was mentioned previously and will be discussed in the following section, where the PC-NCM is studied in the side-mounted configuration, net EOF may have a deleterious effect on the diffusion layers, reducing any length asymmetry because of the difference in fluid velocity on either side of the membrane. This point should be investigated thoroughly in future studies.

In the next section, the performance of a side-mounted NCM is studied for three different channel cross-sections and three different buffer concentrations. Since the model scales all species densities, including that of the fixed charges, to the bulk buffer concentration, it is tempting to compare changing buffer concentration to changing fixed charge density. This should be done with caution. It is well-known that the conductivity of nanochannels at low concentration approaches a limit independent of the buffer concentration due to effectively complete double-layer overlap $[57,66]$. Furthermore, if there is any amount of concentrationdependent charge-regulation, which is physically reasonable (see Ch 2 or $[54,10,2]$ ) this can be anticipated to have an effect. In order to make a more complete comparison, the same experiments should be performed with a top-mounted Nafion device and side-mounted Nafion with different channel sizes. 


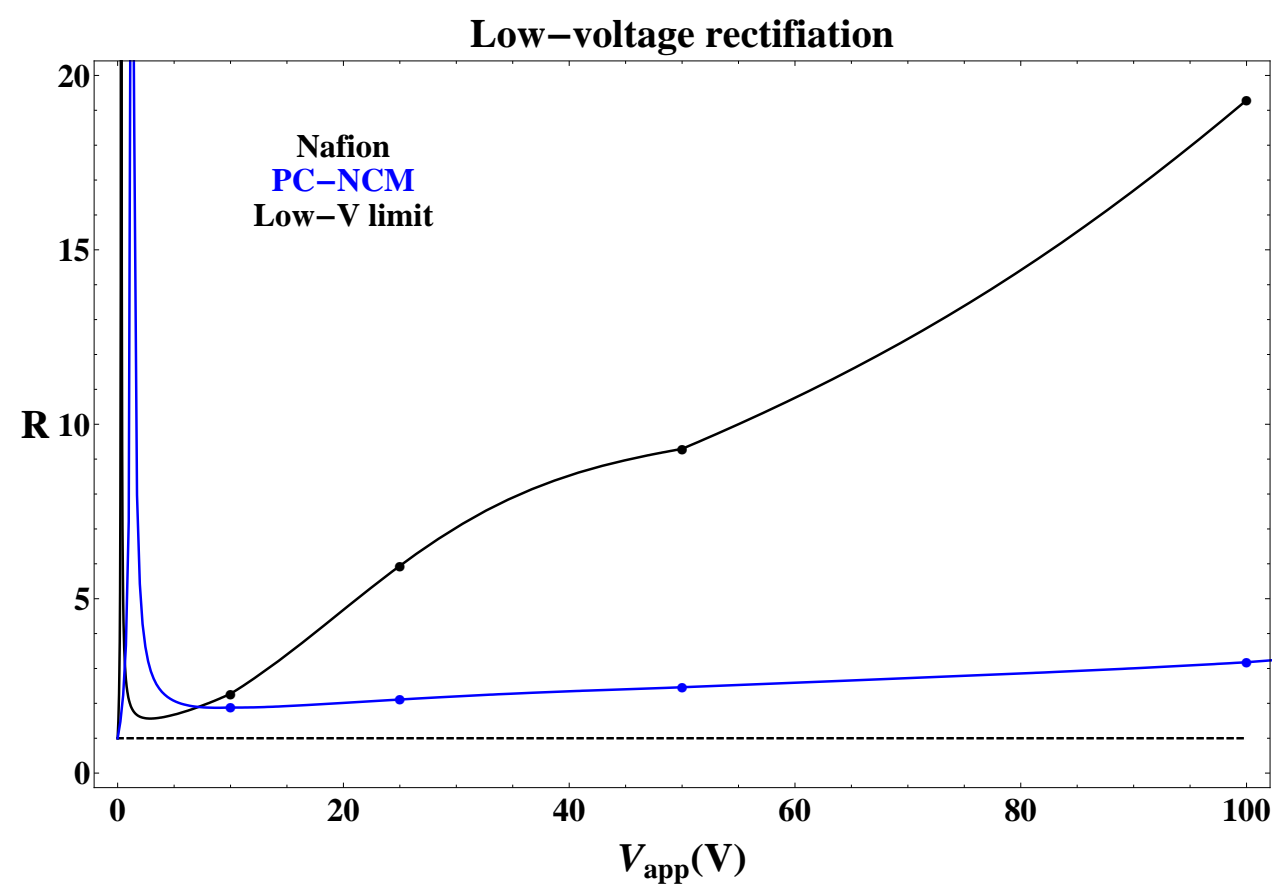

Figure 4.11: Rectification factor for both devices at low voltage with the low-voltage LEN limit indicated by the dashed line. Note that the Nafion case bears a close resemblance to the LEN models below about 50-60V and approaches the classical limit much more convincingly than the NCM. This may be some indication of space-charge/electro-convective effects appearing well below the high voltage range, however still considerably higher than the typical classical limit for an ideal membrane. 


\subsection{Rectification and hysteresis in an asymmetric side- mounted PC-NCM device}

\subsubsection{Rectification}

Figure 4.12 shows the on-state current density vs. time for side-mounted NCM devices with three different channel depths in $2 \mathrm{~V}$ voltage steps. Assuming the inflection of the I-t slope can be used as a reliable indicator of the relative location (in terms of voltage and current) of an absolute upper bound for the classical limiting current, these devices appear to behave as would be expected according to chapter 3. The most asymmetric device, the 12 micron case, has the highest voltage and current density at the inflection, with the 30 micron having the next highest voltage, followed by the 40 micron case. While only one run is shown, similar runs show similar features; the chief difficulty is that, given all of the other variability in the systems, the similarity in size of the 30 and 40 micron channels makes it difficult to distinguish which actually has the higher limiting current.

Nonetheless, this seems to indicate that increasing device asymmetry has the predicted effects; the point at which the system reaches the limiting current in the on-state increases with increasing geometric asymmetry. However, things are not quite so straight-forward, as will be discussed shortly. Before returning to this point, two other points warrant some brief discussion. Note that the inflections here occur about an order of magnitude lower than the inflection in the top-mounted NCM device, which, by virtue of the diameter of the vertical entrance channel, has the least fluidic asymmetry. However, the current densities near these inflections are similar in magnitude to those in the top-mounted case, despite the tremendous difference in applied voltage. Secondly, at least in the 12 micron case, there appear to be additional inflections at higher voltages above the first, a little above $t=200 \mathrm{~m}$ (i.e. $50 \mathrm{~V})$ and again around $t=250 \mathrm{~m}(62 \mathrm{~V})$. It might be worth asking what the relationship is between these inflections and the so-called Teorell's oscillations which are known to occur in similar systems subject to stepped voltages [46].

In Figure 4.13, it is evident that the off-states have significantly higher current densities than the top-mounted NCM device and 12 micron deep side-mounted Nafion device 


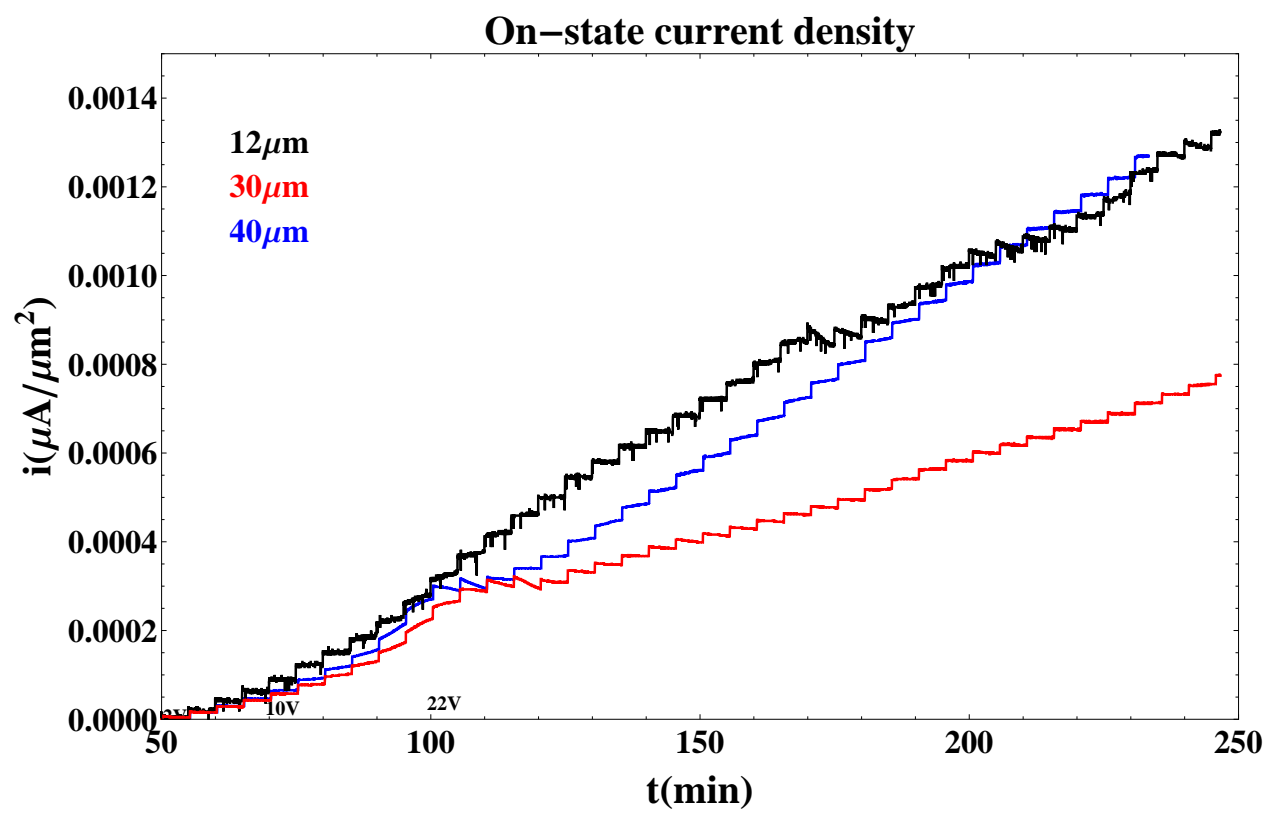

Figure 4.12: On-state current density vs. time for stepped voltages for the three microchannel sizes, $10 \mathrm{mM}$ buffer. $2 \mathrm{~V}$ steps shown. Note the apparent multiple I-t inflections in the 12 micron case. 


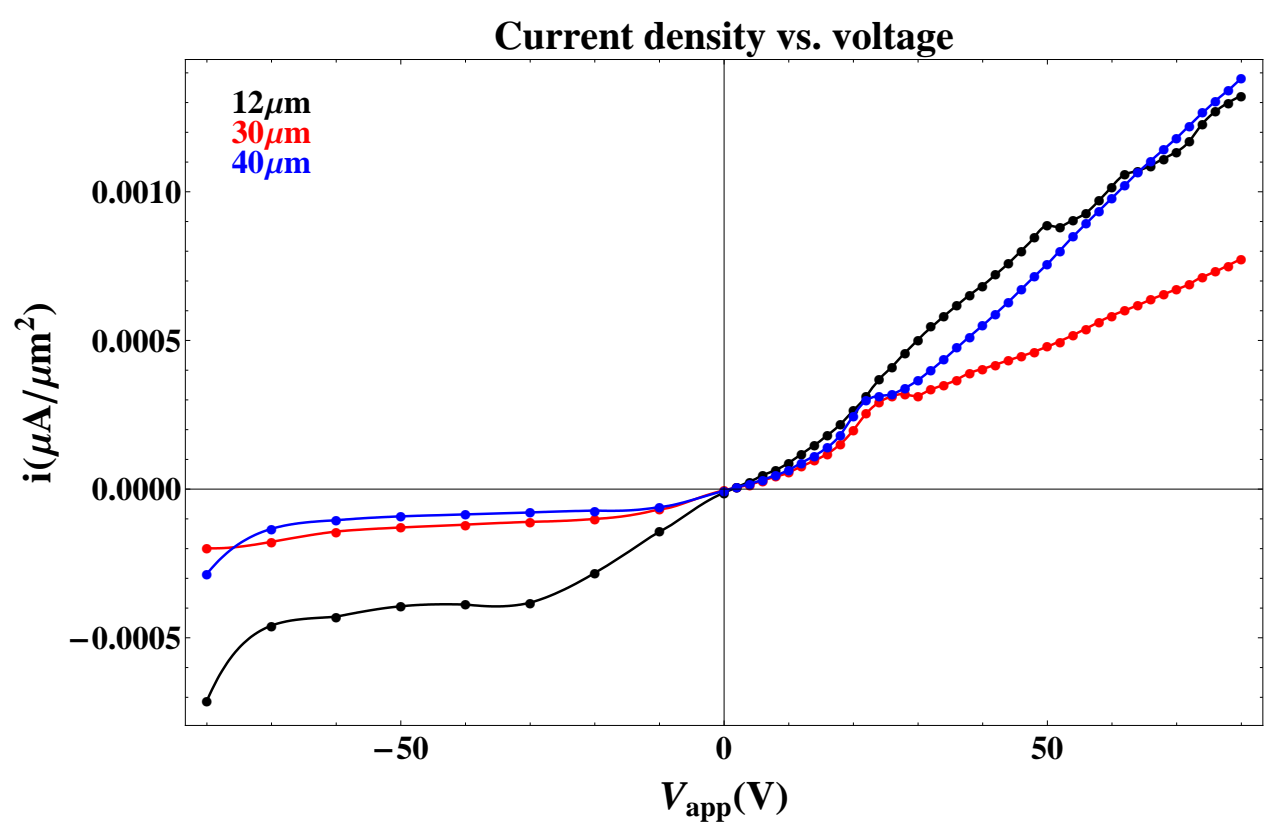

Figure 4.13: Averaged current density vs. voltage for the three microchannel sizes, $10 \mathrm{mM}$ buffer. The 12 micron channel off-state for the side-mounted NCM has an unusually high current density.

(see Figure 4.10) even though the on-state sub-limiting current densities are comparable. This will obviously lead to lower values for the rectification factors, as shown in Figure 4.14. Since the Nafion device was much more well-behaved, it seems reasonable to suspect that net EOF is at least partly responsible for this. A further clue can be gleaned from the fact that the top-mounted NCM, while a less strong rectifier than the side-mounted Nafion device, is still rectifies more strongly than the side-mounted NCM devices with higher asymmetry. In fact, while the positions (in terms of voltage) of the inflections increase with increasing asymmetry - which, by the models in Chapter 3, indicate the rectification factor should also increase- it is clear from the rectification factors that, at least for the side-mounted NCM devices, exactly the opposite is true.

The key piece seems to be the behavior in the off-state. While the on-state behaviors appear consistent with the 1D theory, the off-states clearly are not. It should be noted that 


\section{Rectifiation factor for side-mounted NCM}

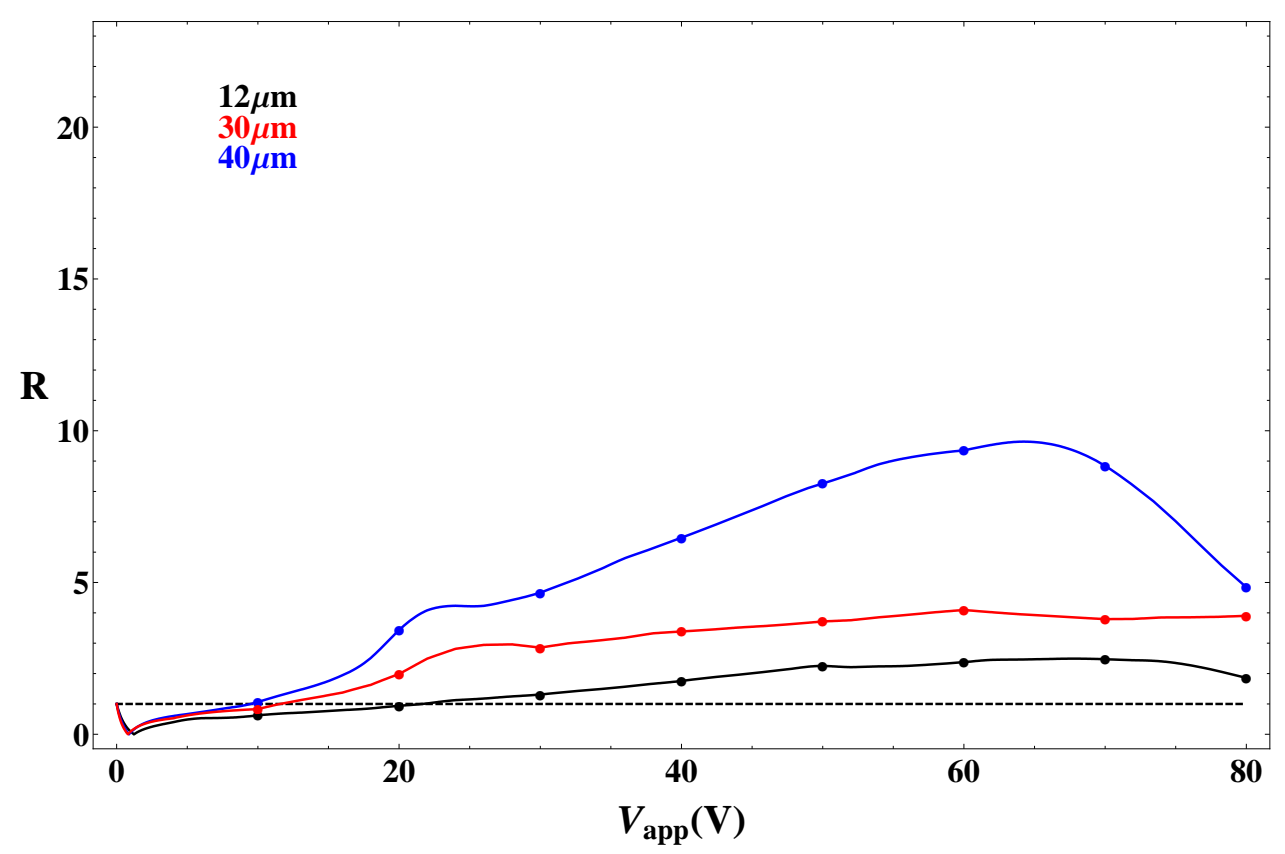

Figure 4.14: Rectification factors for the side-mounted NCM device with three different microchannel depths, $10 \mathrm{mM}$ buffer. The unusually high off-state current densities (by comparison with the top-mounted NCM and side-mounted Nafion membrane) seem to be the source of difficulty. The on-state densities, below the inflections, are comparable to those in the top-mounted device at higher voltages.

in the off-state, both device and membrane EOF are directed outward from the microchannel into the reservoir, which may lead to tenting and issues with the membrane seal against the glass chip. However there is another possible effect which is fundamentally related to the fluidic asymmetry.

In the presence of both fluid flow and electro-diffusion, for sufficiently strong flows, preliminary work shows that the DL length is determined both by diffusive growth and convective mixing [3]. A simple estimate of the convective contribution to the mixing length can be made using the Peclet number.

Conservation of fluid flux yields $v_{r}=v_{\mu} / \Gamma$, recalling that $\Gamma=A_{r} / A_{\mu}$ is the area ratio. Thus the fluid velocity in the reservoir is slower than that in the microchannel. Since the 
Peclet number for electrokinetic systems is 0.5 [50], we can equate this on both sides of the membrane to yield a relationship between the convective contributions to the mixing length. Since $P e=v \ell / \mathcal{D}$, the relationship between convective mixing lengths on either side of the membrane is just $\ell_{r}=\Gamma \ell_{\mu}$, which indicates that for larger degrees of asymmetry, the convective contribution to the mixing length in the reservoir will be greater than that in the microchannel. Thus, it seems as though changes in the mixing length due to convection compete against those due to diffusion in asymmetric systems. Even if the rectification is chiefly due to flux-focusing rather than DL length asymmetry, convective mixing will act to reduce the overall rectification strength by providing for a larger convective mixing length in the reservoir than the micro-channel. This argument, while suggestive, is by no means rigorous and requires further investigation before any definitive conclusions may be made.

\subsubsection{Varying bulk concentration}

As mentioned previously, since all charge densities are scaled by the bulk electrolyte concentration, it is tempting to consider the behavior of the NCM at several values of bulk concentration as a means of testing the applicability of the 1D theory. However, as evident in the preceding experiments, the situation is not so simple. The I-V plots shown in Figure 4.15 agree well enough with the predictions of the 1D theory. In all cases, the current density for the highest $\mathrm{N} /$ lowest concentration is the lowest, and the converse holds, at least for voltages up to around 20V. However, the inflection points (not shown) taken as indicators of the relative voltages at which LEN breakdown occurs are not consistent at all. There are several reasons this is not too troubling. First, according to the theory in chapter 3 , the asymmetry is anticipated to play a larger role than charge density in determining this behavior. Secondly, the I-t inflections, while indicative of electro-convective processes related to the extended space charge, likely involve other factors, such as net EOF. This might help explain the difference in response between the side-mounted NCM devices with the top-mounted NCM, which is markedly less asymmetric and operating at considerably higher voltages.

The rectification factors plotted in Figure 4.15 show behaviors comparable to other 


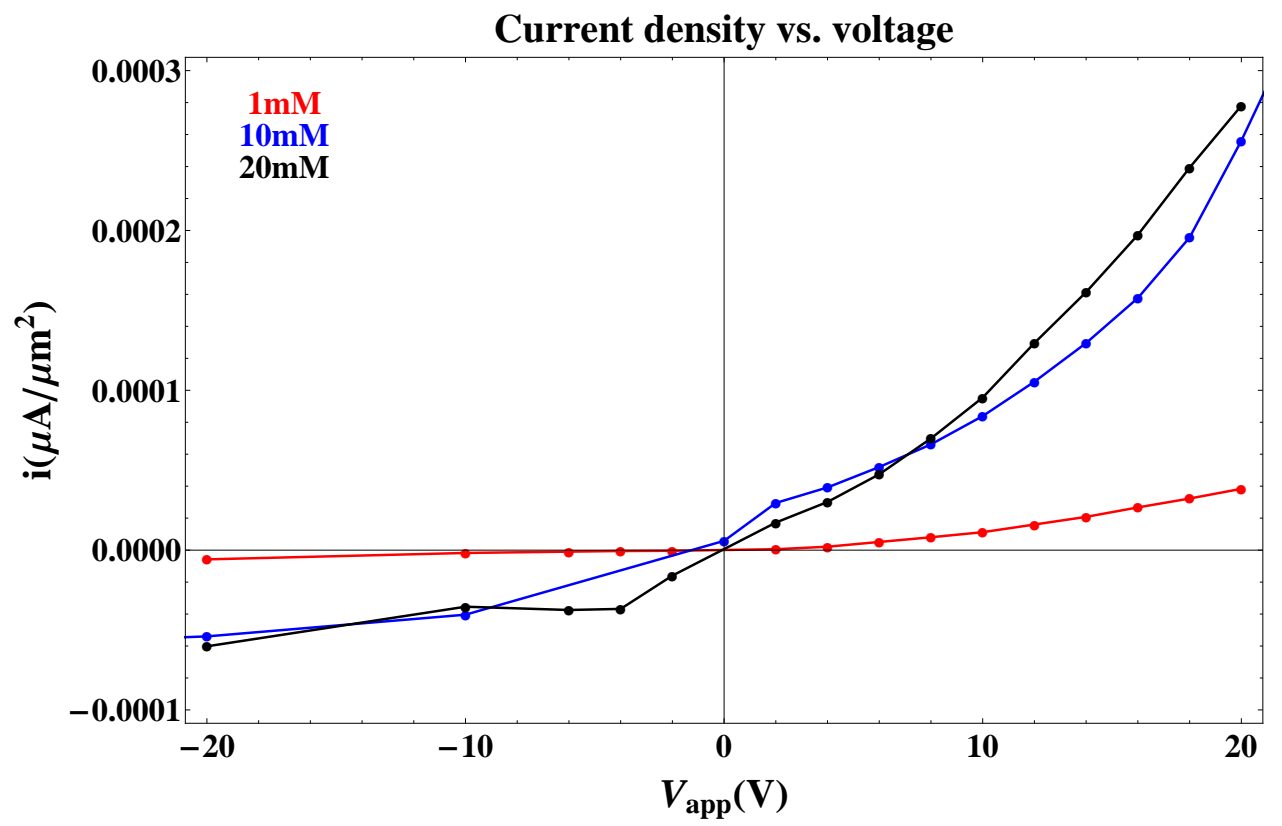

Figure 4.15: Averaged I-V curves for the 40 micron side-mounted NCM device at three different concentrations. The I-t inflection point was not visible on the $1 \mathrm{mM}$ case, but occurred between $18 \mathrm{~V}$ and $20 \mathrm{~V}$ for the $10 \mathrm{mM}$ and $20 \mathrm{mM}$ cases. 


\section{Rectifiation factor for side-mounted NCM}

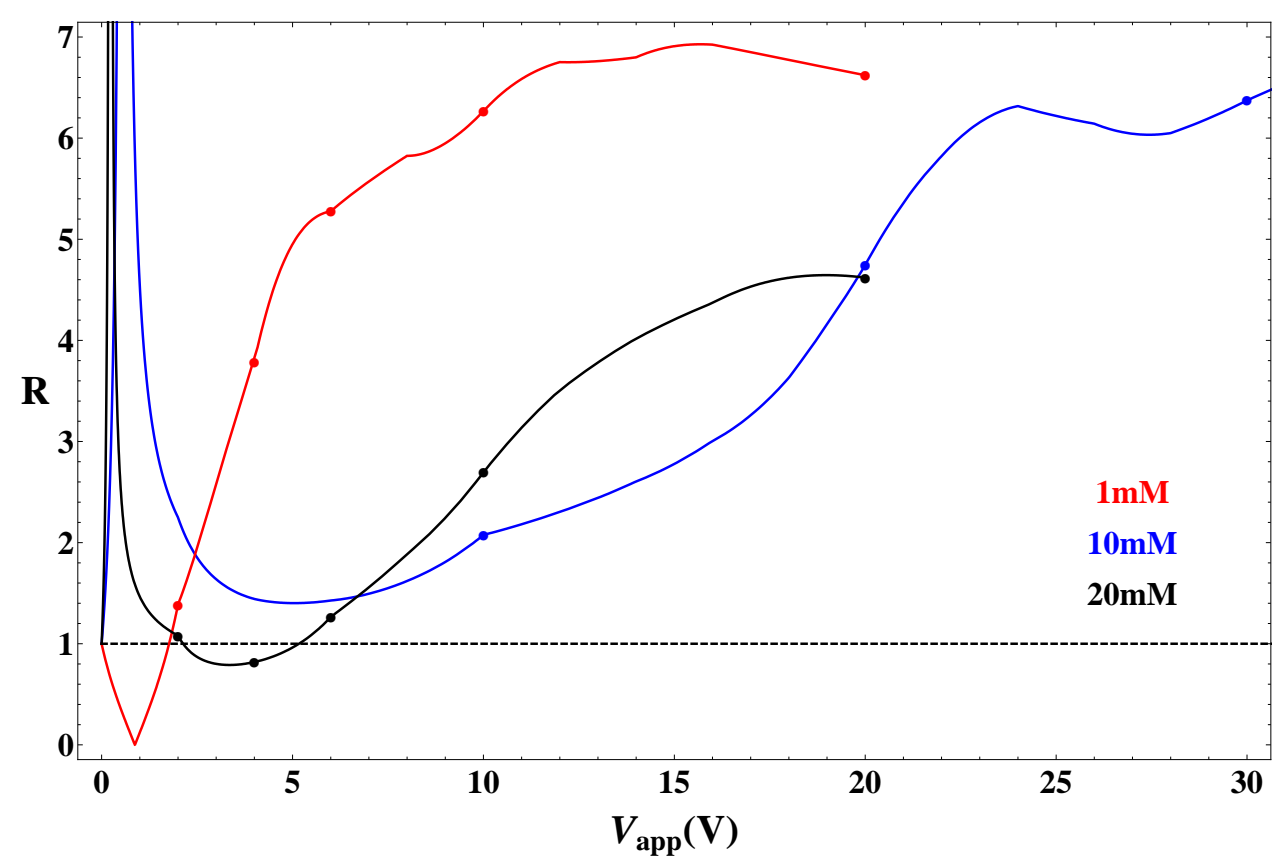

Figure 4.16: Rectification factors for the 40 micron side-mounted NCM at three different concentrations. The behavior near $V=0$ is an artifact of the interpolation. The dashed line shows the theoretical low- $\mathrm{V}$ limit of chapter 2.

experiments, and at least superficially for the lowest voltages, appear to agree with the 1D theory and the assumption that changing the bulk concentration is equivalent to comparing different values of fixed charge density. At the lowest voltages, it appears as though the highest concentration, which under these assumptions corresponds to the lowest charge density, has the highest rectification factor. However, due to the lack of data at extremely low voltages and the resultant nature of the ratio of interpolated I-V curves, this conclusion cannot be trusted.

In this case, while EOF is expected to play a role, there is yet another issue, mentioned earlier. For nanochannels, it was shown in chapter 1 that the concentration of electrolyte in the channel can affect the surface charge. While it is generally accepted that simple sitedissociation is not the primary mechanism responsible for the NCM surface charge ${ }^{3}$, it is

\footnotetext{
${ }^{3}$ The content of this discussion from personal correspondence with Michael Hickner of Sandia Laboratories.
} 
likely the case that the actual mechanism, possibly involving the adsorption of negative ions from solution, does in fact depend on both the concentration and $\mathrm{pH}$. Another potential issue here is similar to what has been observed in other nanochannel systems [57, 10, 65]; that at extremely low concentrations, the overlapped double-layers dominate and the conductivity saturates at a value independent of the bulk concentration. As the limiting current is approached in either the off- or on- states, the formation of a strong depletion zone at one or the other ends of the channel might be expected to modify the local Debye length. Thus, for either very low concentrations or voltages near/above those corresponding to the limiting current, these effects may play an important role. To understand transport across a range of conditions, this issue must be explored further both theoretically and experimentally.

\subsubsection{Chemical rachet: electrokinetic hysteresis}

There is at least one effect in the literature that the 1D theory does a reasonably good job of explaining without extensive hand-waving or overbearing use of caveats: the hysteresis effect. This effect has been observed in similar systems [5] and is relatively simple to understand in terms of concentration polarization. Hysteresis occurs in these systems because residual concentration polarization from prior measurements can impose a concentration gradient across the membrane. The concentration polarization due to subsequent measurements must effectively be super-imposed upon this residual gradient.

In the experiments of Yossifon and Chang using a symmetric device, the voltage is incrementally increased, then decreased. The two curves do not rest on top of one another, but exhibit a clear hysteresis effect. Here, since the devices used are asymmetric, the changing microchannel concentration dominates the response of the device. The voltage is stepped up from an off-state negative voltage through positive on-state voltages. During the off-state runs, the degree of depletion in the microchannel decreases with each step in voltage. This may be the reason behind the small decaying transients apparent in some of the I-t curves at low positive voltage, and may play some role in the fact that the measured off-state responses tended to be the most problematic. To be sure, experiments could be carried out in which the voltage is stepped down from zero and compared to the increasing voltage steps. One 
might expect that there is some residual enrichment on the reservoir-side, but this can be expected to dissipate quickly since there will generally be a steeper gradient (assuming the DL is shorter.) Besides, the enhanced reservoir concentration is not as critical in determining the response of an asymmetric device.

During the on-state runs, the voltage is increased step-wise, and the degree of enrichment in the microchannel increases with each step. This will not affect the response of subsequent steps, except in as much as we might anticipate the system reaches a quasi-steady state corresponding to the fully-developed CPL for that voltage more rapidly than if that particular voltage were applied to a fresh system. However, if a new run is performed immediately after the completion of the first run, i.e. returning to the lowest off-state voltage, it is done with a microchannel concentration that is enriched with respect to the reservoir. Even for reasonably long intervals (up to about a minute or so) between subsequent runs, the microchannel enrichment does not dissipate appreciably. If in fact the microchannel does have a longer DL than the reservoir, hence a less steep concentration gradient, the relaxational diffusion current will be smaller in the microchannel. So, the net effect is that the microchannel conductivity increases with each subsequent run.

This effect can clearly be seen in Figure 4.17, where the initial current density at the lowest voltage is higher for each subsequent run. What is also very clear in the figure is that the on-state seems to be relatively un-affected. The effect can be simulated by calculating three I-V curves, in this case using the ideal model for simplicity, wherein the microchannelside bulk concentration is increased from run-to-run while keeping the reservoir bulk fixed. The microchannel bulk boundary value for the $n+1$ th run was chosen to be half of the average of the peak on-state concentration at the membrane and the microchannel bulk value for the $n$th run. While somewhat arbitrary, this parameter choice is representative of the fact that the concentration profile from a prior run will dissipate somewhat between switching measurements. In the experiments, the time between runs was not carefully controlled. In fact, these experiments were not exactly designed to test this effect. We were actually trying to save time in performing the runs for analysis in the previous sections, and were initially troubled by the apparently severe irreproducibility in these cases and almost threw the data out; applying the same voltage twice to the system without making any apparent changes 


\section{Current density vs. voltage, $40 \mu \mathrm{m} 1 \mathrm{mM}$}

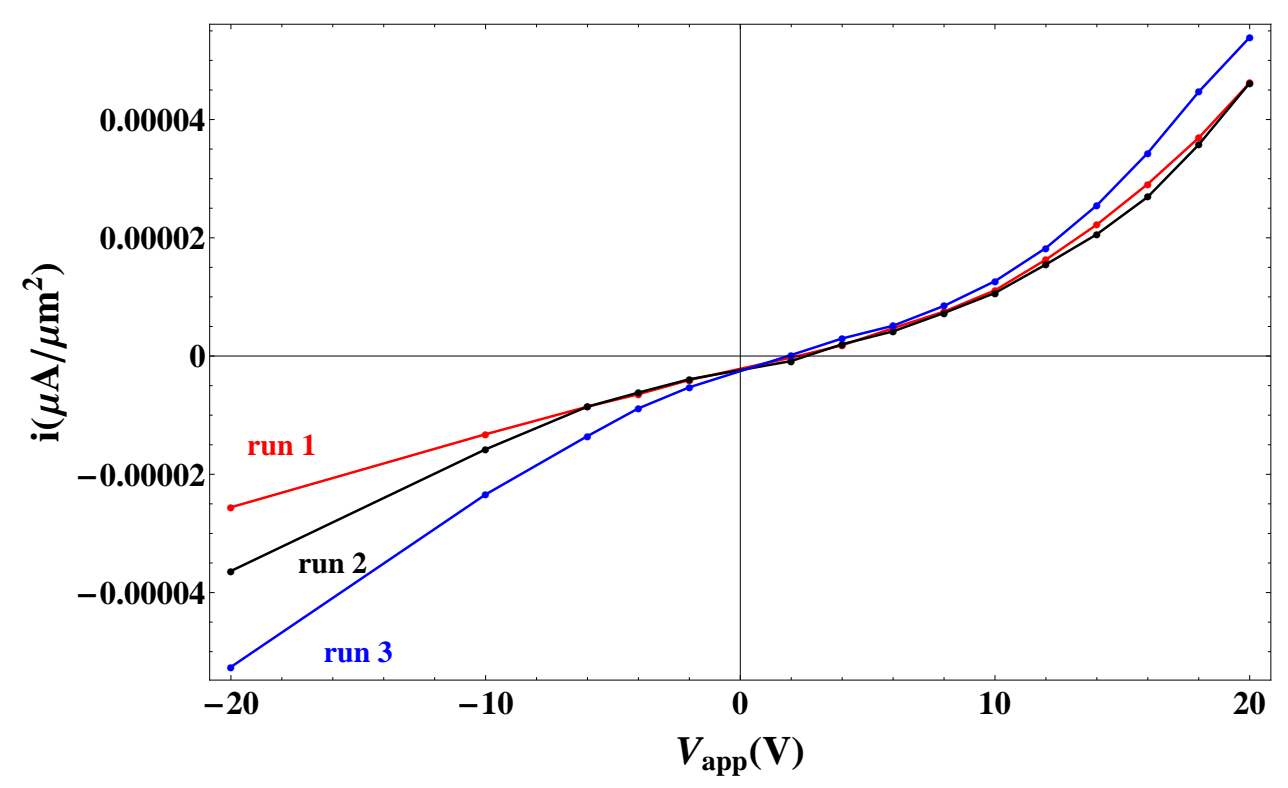

Figure 4.17: Experimental observation of chemical ratchet effect in a 40 micron side-mounted device at $1 \mathrm{mM}$ buffer concentration. The runs were performed in (somewhat) rapid succession, starting at the largest negative voltage, where the system sat for 10 minutes (first five minutes not shown) before stepping up through the sequence of voltage. 


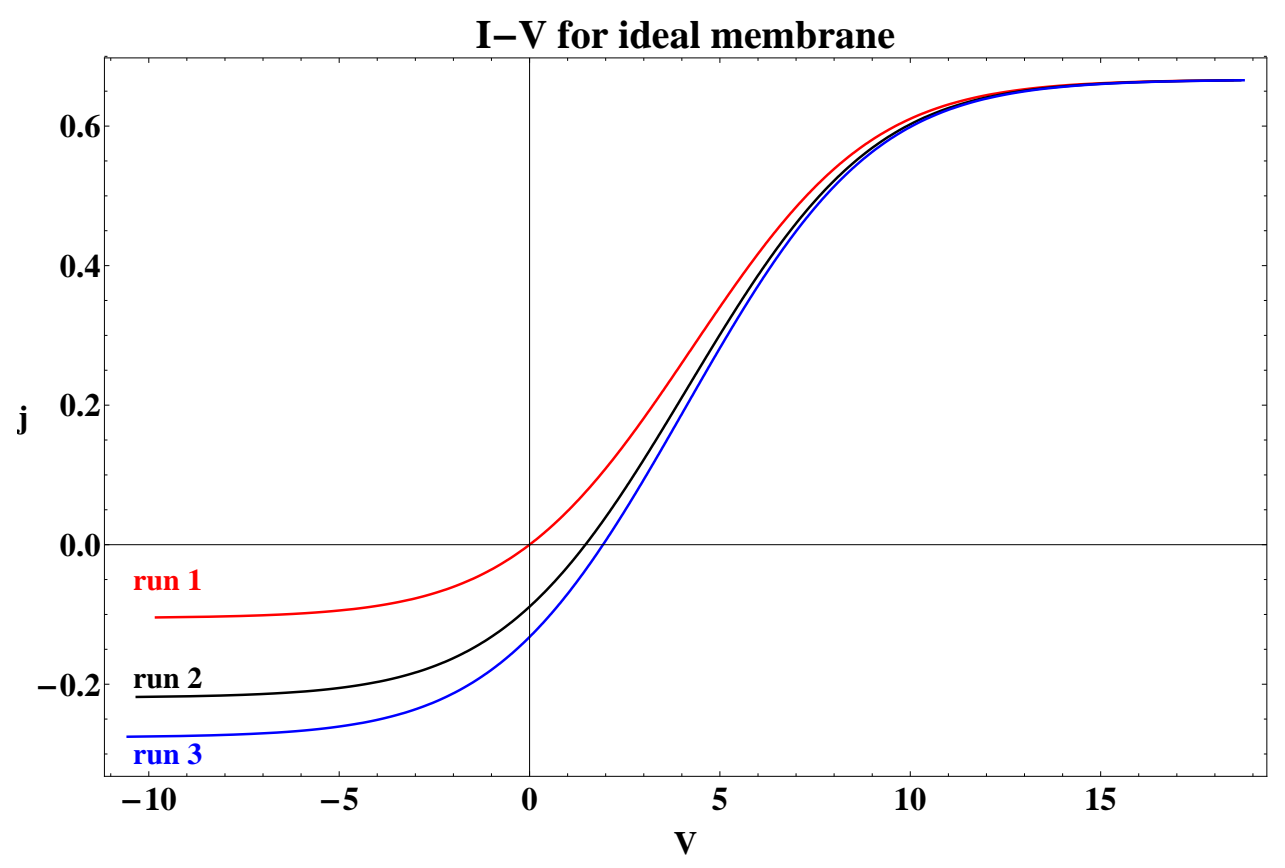

Figure 4.18: Calculated chemical ratchet effect using arbitrary parameters and the ideal model to demonstrate the basic mechanism

should yield the same value. However, upon thinking a little, it becomes quite clear what is happening, and brings great relief that the theory of chapter 3 has provided at least one un-ambiguous explanation for the NCM/NMI experiments.

\subsection{High-voltage off-state analyte concentration}

A number of studies have looked at NMI-based analyte preconcentrators [43, 41, 26, $15,11,33,34,61,16]$. As mentioned in the introduction to this thesis, there are two distinct modes of concentration. These modes, in systems with fluidic asymmetry such as the NCM/NMI correspond to the off- and on- biased rectification states. Again, as was discussed previously, the on-state corresponds to simple concentration polarization, occurs in symmetric devices as well [43], and does not depend on effects related to the extended space 
charge. Two general types of explanation have been offered for the off-state concentration devices, and it seems reasonable to suppose that, to a greater or lesser degree, both can act simultaneously in the same system. The first mechanism can be described by $1 \mathrm{D}$ averaged models and corresponds essentially to a competition between net EOF and electrophoresis. This explanation, along with comparison between experiment and modeling, has been offered by (at least these) two groups, [41,33,34]. One note is that this effect does not seem to require extended space charge and may occur at lower voltages. The other effect involves trapping by vortices associated with the extended space charge $[26,61,16]$, though from the geometries involved, i.e. nanochannel interconnects between parallel microchannels, thus involving sharp corners, it is not clear whether these vortices are due to Duhkin's EOF of the 2nd kind or vortices of the Rubinstein-Shtillman-Zaltzman type.

In this section, the off-state concentration effect will be studied for extremely high voltages in the NCM/NMI. The effect has been observed at lower voltages as well (see [24], and from that data, as well as the I-t curves presented earlier in this chapter, it seems as though there may be distinctly different regimes of operation. Here, no attempt is made to clarify the concentration mechanisms, but instead a quantitative description of the change in plug position and width with time is presented, intended for use in future modeling studies. One question which begs serious consideration is whether or not the region near the analyte plug itself is not locally electro-neutral. Some preliminary work ${ }^{4}$ suggests that this is indeed the case, and that the charge separation even persists for a brief period after the voltage is switched off. This was ascertained from an averaged 1D analysis of the band-broadening of the analyte during the first few seconds after switching off the voltage. However, the results were somewhat ambiguous and require further investigation.

\subsubsection{Some notes on use of fluorescent imaging to obtain informa- tion about concentrations}

The equipment and methods used here are essentially the same as those in [24], and the interested reader is referred to that paper for more details. However a few experimental

\footnotetext{
${ }^{4}$ Unpublished work presented at the 2009 APS March meeting
} 
issues relevant to the present analysis are discussed. The bulk concentration of the Fluorescien analyte is $20 \mu \mathrm{M}$, so in bulk the contribution of the analyte to solution conductivity is negligible and it can be treated essentially as a passive background molecule. However, this is not necessarily the case at high concentrations. Thus at low concentrations and for quasi-equilibrium conditions (near the interface, i.e. under which extended space charge is negligible) the analyte concentration can be used as a reasonable indicator of the overall conductivity and structure of concentration polarization, this cannot be tacitly assumed at high-off state voltages wherein both extended space charge due to the non-classical effects can be anticipated as well as possible LEN violations due to extreme conductivity gradients may play some role.

Another issue to keep in mind is that at high concentrations the microscope images can become saturated, so that further increases in concentration are not measurable. Thus, the results shown here cannot necessarily be used for a rigorous quantitative analysis of the spatiotemporal changes in solution composition. In the vicinity of very high concentrations, it is difficult to determine the extent and distribution of intermediate concentrations; the effect is something like staring into oncoming headlights on a dark road, the periphery becomes indistinct. Unfortunately, much of the shape of the concentration front which might indicate the presence of vortices or other electrokinetic mixing-type instabilities are obscured by the near-by regions of high concentration. To overcome this, the microscope images were post-processed using Mathematica and an intensity-based contour map obtained. For observations of plug position and width, as well as estimates of the depletion zone growth, these images were averaged across the channel cross-section and the 1D averages analyzed.

\subsubsection{Analyte concentration process at $-800 \mathrm{~V}$}

Figure 4.19 shows several frames of contour plots of the intensity rendered from an epifluorescence microscope image of a microchannel which had initially been filled with a $20 \mu \mathrm{M}$ concentration of Fluorescein dye. The dye was allowed to diffuse (approximately) evenly through the channel before a large negative voltage was applied at $t=2.166 \mathrm{~s}$. Similar concentration behavior has been observed at lower voltages as well (see [24].) However this is 
interesting in particular because it occurs in the extreme range of off-state voltages, where, at least in the top mounted device, the transient behaviors occurring at intermediate negative voltages are not apparent in the I-t curves Figure 4.4.) While the data in Figure 4.4 were not taken with any analyte, as is apparent in Figure 4.20 the behavior is qualitatively similar. From some preliminary steady-state work (see Ch 1. and also as reported in [7],) we anticipate that the major effect of added supporting analyte is to alter the magnitude of the net current. However, the characteristic response time does depend on an effective diffusivity and this can be anticipated to change the overall time-scale of the response somewhat.

\section{Six important identifiable temporal regions}

From observation of movies of the concentration process and the Figures 4.19,4.20,??,4.22, several different events are apparent. The interesting transient behavior occurs in the first 15-20 seconds under applied voltage, thereafter the plug concentration increases, the halfwidth decreases while the measured current and position of the plug center-of-mass remain approximately constant. Presumably the overall plug height continues to increase, however saturation of the microscope image makes this difficult to ascertain the peak height accurately. The initial transient phase may be divided into six different temporal regions wherein the system exhibits various responses related to, among other things, electrokinetic instabilities, electro-convective vortex formation, and possibly a high-voltage DL length selection process.

\section{REGION 1: $\sim 2.16 \mathrm{~s} \leq t \leq \sim 5 \mathrm{~s}$}

During initial phase starting with the application of $-800 \mathrm{~V}$ at about $2 \mathrm{~s}$, the initially uniform analyte rapidly contracts within a few hundredths of a second (sample rate at 0.057 sec per measurement) and, with little perceptible change in the image, remains close to the membrane, but still diffuse. The microscope image shows a slightly concentrated band and no evidence of vortices. Only very slight depletion is apparent in the sense that the maximum 
Time $=4.731 \mathrm{~s}$.

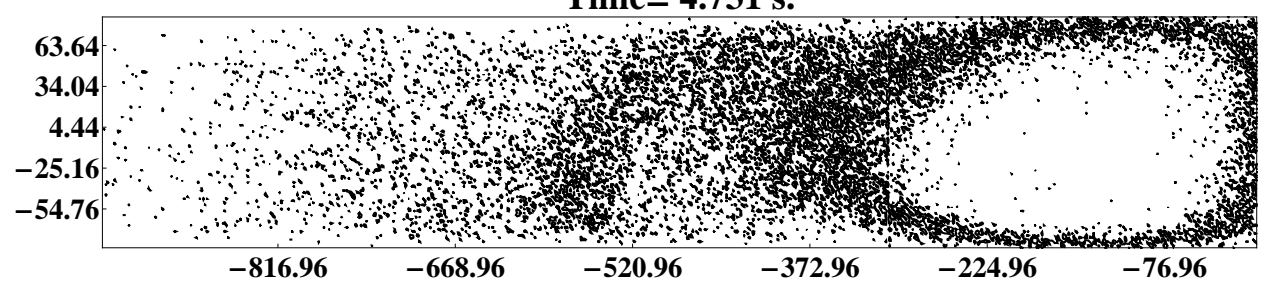

Time $=5.586 \mathrm{~s}$.

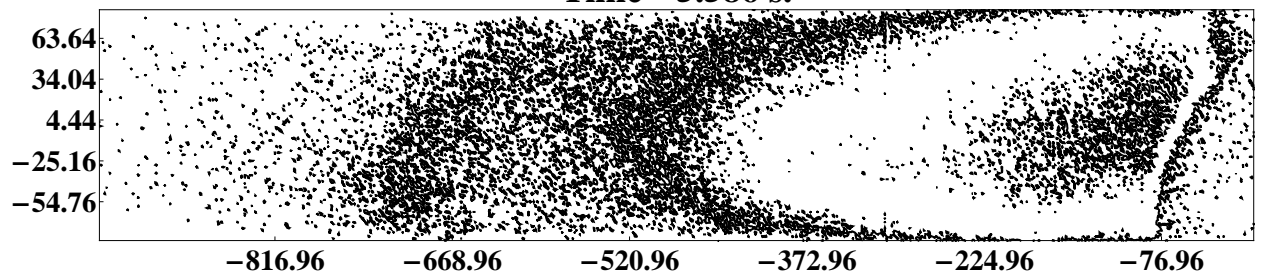

Time $=6.954 \mathrm{~s}$.

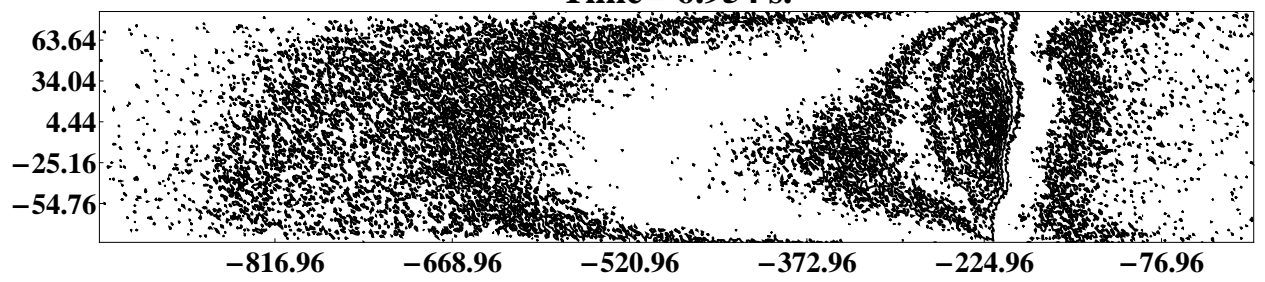

Time $=8.094 \mathrm{~s}$.

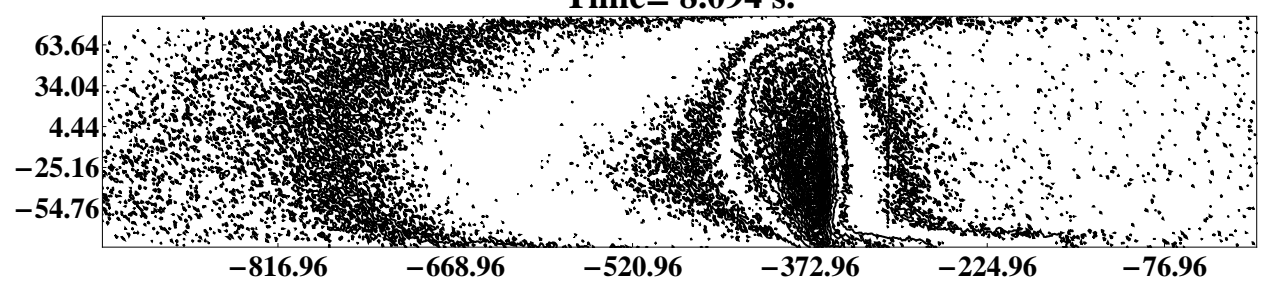

Time $=12.369 \mathrm{~s}$.

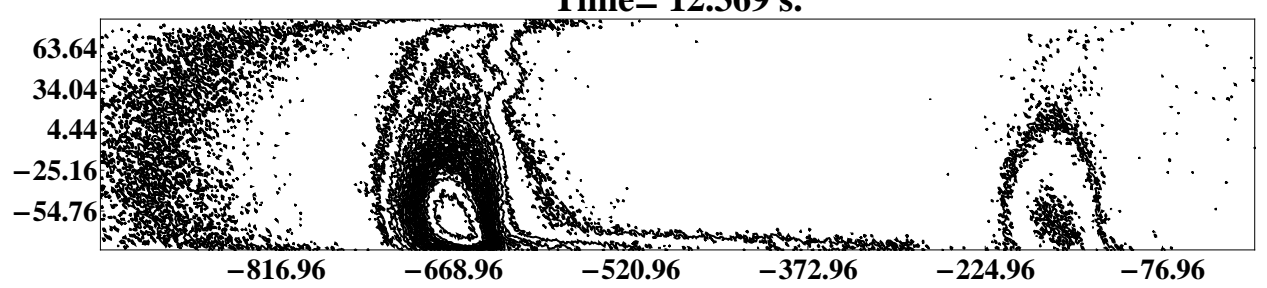

Figure 4.19: Post-processed contour intensity maps of fluorescent microscope images at several time frames during the initial transient phase of analyte concentration in the highvoltage off-state. The membrane is located at the far right side of the figure and the distance from the membrane back through the channel given in microns. Undulations, characteristic of vortex-like instabilities in the depleted region, are evident at the side of the plug closest to the membrane. Note also these images do not show the full channel width, this reduced box was used to improve the resolution of the image. Lastly, in the last frame, an apparent ghost image due to image saturation appears trailing the actual plug by about 400 microns. 


\section{I-t at $-800 \mathrm{~V} w / 20 \mu \mathrm{M}$ Fluorescein}

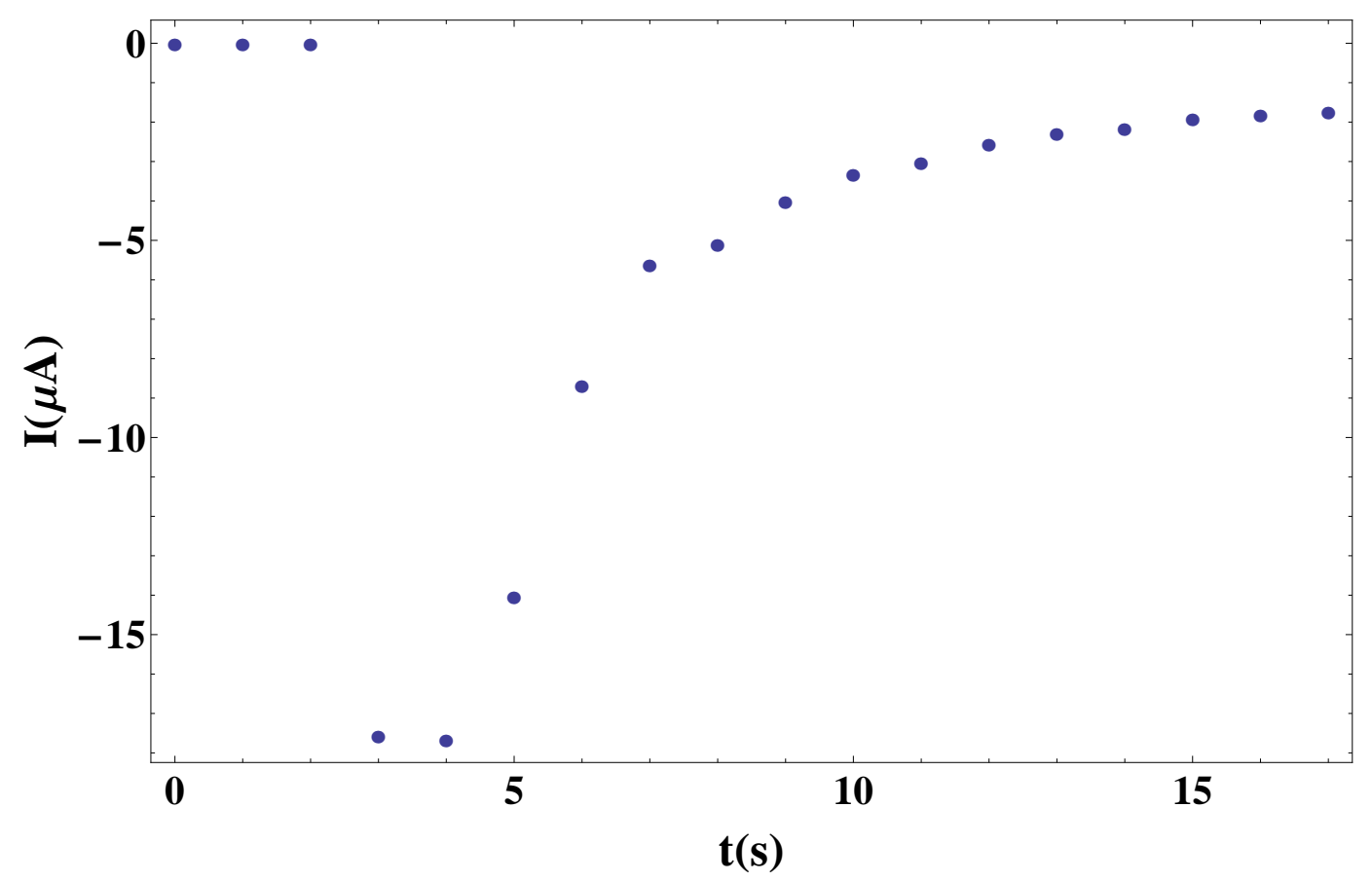

Figure 4.20: Plot of the transient current pulse during this time period. By about 15 seconds, the system achieves what appears to be a quasi-steady value. The initial transient corresponds to the formation of the depleted region. 


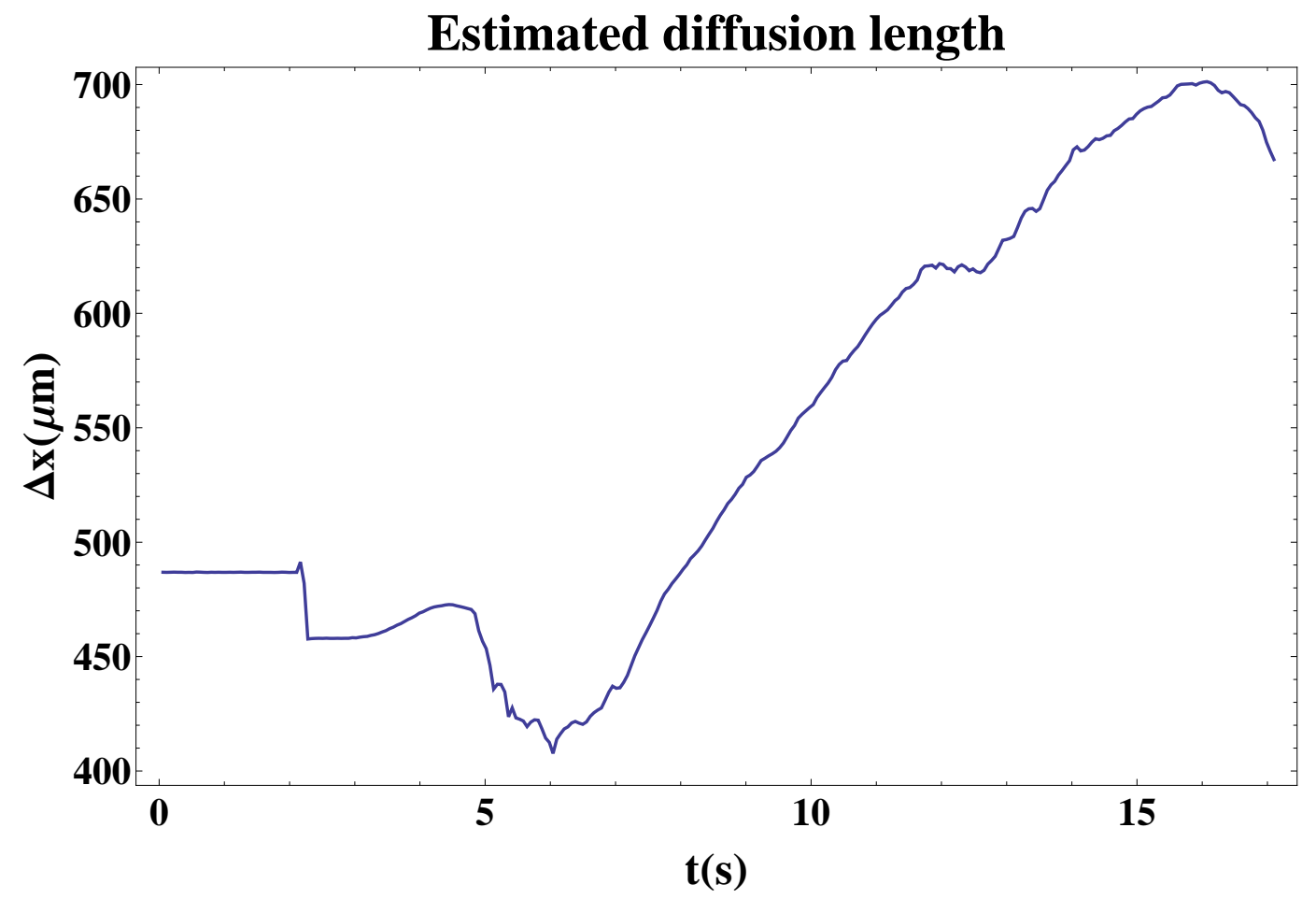

Figure 4.21: Plot of the position of the plug mean vs. time obtained from a 1D average of the data presented in Figure 4.19. The change in the position of the mean may be used as a very rough estimate of changes in the spatial extent of the depleted region. Note that several distinct regimes of behavior are apparent in this figure. After about 17 seconds, the plug position remains roughly constant for the duration of the experiment. 


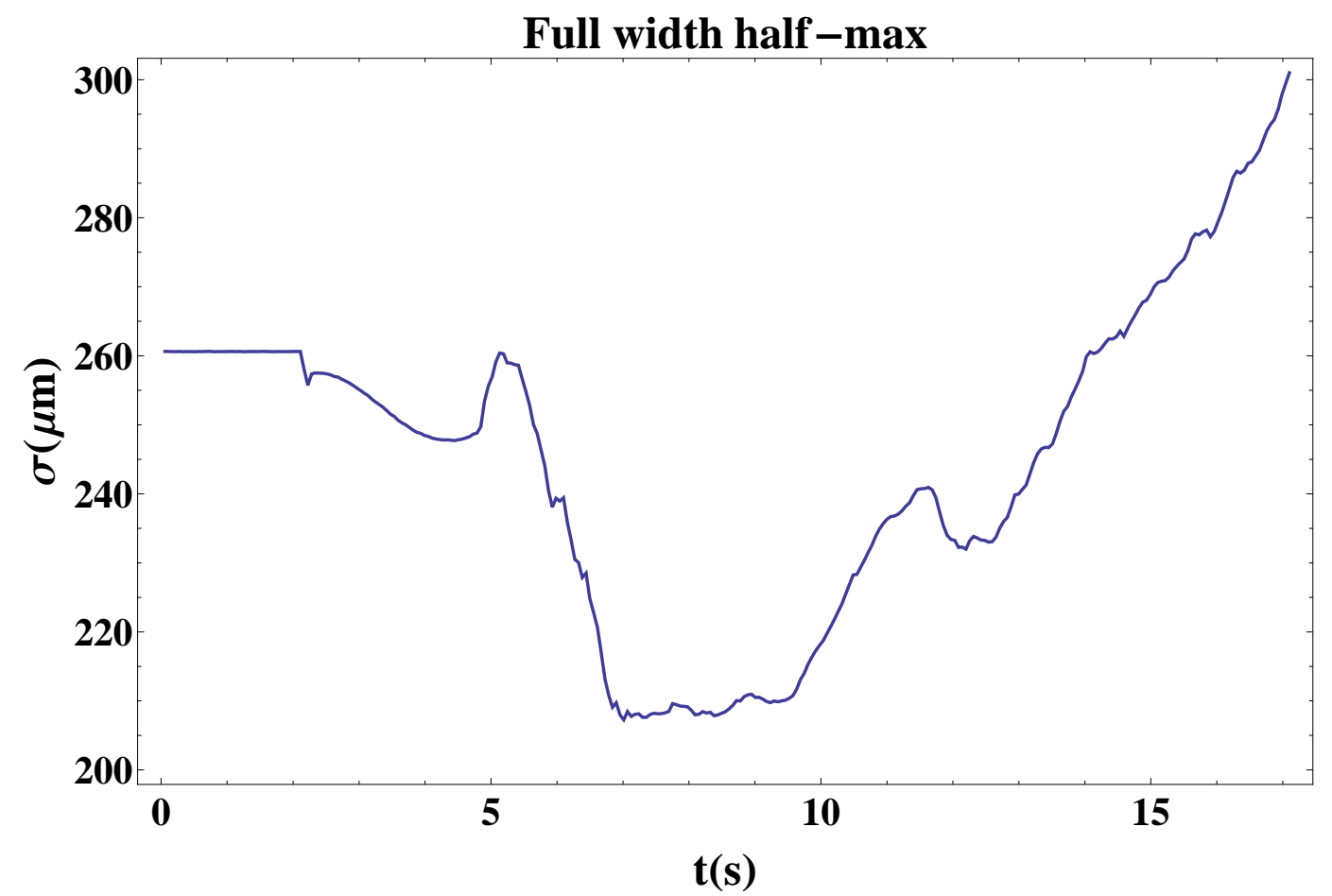

Figure 4.22: Plot showing the variance, proportional to the full-width half-maximum, of the plug. These were extracted from the 1D data by calculating the moments of the $1 \mathrm{D}$ averaged distribution. The rapid contraction in both this plot and the previous one are indicative of the rapid build-up of an extended space charge adjacent to the membrane. 
intensity is not at the membrane surface, but further into the microchannel.

In the same time as the initial contraction, the current drops rapidly to a minimum around $-18 \mu \mathrm{A}$ and remains around this minimum for about 1-2 seconds before the begins to decrease. The rapid increase in magnitude is most likely due to the formation of extended space charge, which appears to happen on the order of 0.01s. The subsequent slow decrease in magnitude would be consistent with the formation of a depleted layer. While, for the sake of simplicity and convenience, the discussion will often refer to the universal scaling dynamics mentioned elsewhere in this thesis and the references, it should be well noted that, at these voltages, the depletion layer growth will likely be governed by the Stephan-problem like dynamics as discussed in rigorous detail in Ref [49]. These time-scales can be anticipated to be much faster than those of electro-neutral diffusion occurring at lower voltages $[1,52]$. During this time, the analyte plug rapidly contracts, as evident in Figure 4.22 while the mean of the analyte distribution moves outward, consistent with the notion that the anticipated positive space charge would attract the negatively charged analyte.

\section{REGION 2: $\sim 5 \mathrm{~s} \leq t \leq \sim 7 \mathrm{~s}$}

At about 5 seconds, another (less) rapid contraction of analyte followed by evidence of depletion zone vortices similar to those observed by Yossifon et. al. [64] and predicted by Rubinstein et. al. appears. There is no observable stable vortex array at any point and the position of a now-sharp DL edge, defined by what appears to be fluctuating vortices, begins to move outward. The magnitude of the current declines less rapidly than the prior increase, from $-15 \mu \mathrm{A}$ to around $-5 \mu \mathrm{A}$ in about 3 seconds. The position of the analyte reaches a minimum at just under 6 seconds and begins to move outward. The time-dependence of the motion of the mean is roughly parabolic about this minimum. After a rapid increase centered around 5 seconds, the analyte variance rapidly decreases by over 60 microns in about 2 seconds. Whether this is related to vortices, or the motion of a concentration shock [33] is unclear. 
REGION 3: $\sim 7 \mathrm{~s} \leq t \leq \sim 10 \mathrm{~s}$

At this point, the analyte has an evident front, which is moving away from the membrane, while more analyte presumably moves into the microchannel itself via net EOF and appears to concentrate at this front. Undulations in the front edge, which appear similar to patterns seen in the vortex observations of Yossifon et. al, are most clearly evident during this period. Meanwhile, the current undergoes a much slower decrease in magnitude, appearing to asymptotically approach some steady-value. Concurrent with the apparent outward motion of the front, the mean of the analyte moves outward more rapidly than can be accounted for by pure diffusion of the analyte. By assuming the simple estimate $\Delta x \sim \sqrt{D_{\text {eff }} t}$ holds reasonably well between about 7 and 10 seconds and noting the DL increases by about $110 \mu \mathrm{m}$ in $3 \mathrm{~s}$, the estimated effective diffusivity is $4033 \mu \mathrm{m}^{2} / \mathrm{s}$. The variance of the plug remains approximately constant during this period, with a very slight increase. This itself is somewhat interesting, since this implies the plug is not diffusing appreciably.

REGION 4: $\sim 10 \mathrm{~s} \leq t \leq \sim 12 \mathrm{~s}$

During this period, the plug appears to move forward, with some apparent increase in overall intensity, and little to no visible evidence of vortex-patterns at the moving front. The current decreases slowly in magnitude at this time while the plug mean position continues to move outward at approximately the same speed as before. However, the plug width rapidly increases by about 20 microns in 2 seconds, which does correspond approximately to the diffusivity of Fluorescein as measured by $\mathrm{CE}$ in an identical buffer solution ${ }^{5}$ This may also correspond to the accumulation of additional analyte brought in from the reservoir, which is consistent with the idea that net EOF effectively 'delivers' the sample to the membrane, counter to the electrophoretic velocity. This offers some support of the ideas presented in Plecis, et. al. [41].

\footnotetext{
${ }^{5}$ Unpublished C.E. measurement (K. Kelly, 10/18/07) gives a value of approximately $170 \mu \mathrm{m}^{2} \mathrm{~s}^{-1}$ with a standard deviation of about $21 \mu \mathrm{m}^{2} \mathrm{~s}^{-1}$
} 
REGION 5: $\sim 12 \mathrm{~s} \leq t \leq \sim 13 \mathrm{~s}$

Interestingly enough, during this period, there is an apparent re-appearance of vortexlike undulations at the depleted edge of analyte band and wrapping around the top side of the plug, which could be possible evidence of vortex annihilation-creation processes. Alternatively, this may be related to the Rayleigh-Taylor-like electro-viscous instability of Chen et. al. [6], which depends upon a critical concentration gradient. The secondary image appearing in Figure 4.19 is believed to be a ghost image in microscope camera due to image saturation. During this time, the slow asymptotic decline in the magnitude of current continues. Notice that this corresponds to a brief plateau in the plug position while the plug width undergoes a rapid contraction followed by resumed expansion, which would be indicative of some kind of electro-convective process such as might occur as a result of one of these instabilities.

REGION 6: $\sim 13 \mathrm{~s} \leq t \leq \infty$

In this final phase of the transient period, the plug appears to reach an asymptotically steady position, further movement out from membrane slows until no longer observable. There is little visible evidence of vortex behavior in images during this phase (not shown) and the current is essentially constant. The plug mean position appears to reach a maximum distance from the membrane, then moves back towards the membrane slightly before remaining approximately steady while the plug width continues to increase un-abated.

\subsection{Summary, conclusions, future work}

\subsubsection{Summary and conclusions}

\section{Applicability of 1D theory}

Overall, the NCM/NMI experiments as a test of the applicability of the 1D theory are inconclusive. The on-state device behavior seems for the most part to agree with the qualitative, and even to some degree, semi-quantitative predictions of the theory. The theory also 
seems to provide an adequate, albeit qualitative, explanation of hysteresis in these systems. However the off-state behavior, particularly with the side-mounted NCM devices, does not appear to be consistent with expectations from 1D LEN electro-diffusive theory at all. There are two likely sources of this difficulty: appearance of extended space charge and attendant electro-convective effects and net EOF (of the first kind.)

Since the 1D theory predicts lower limiting currents for the off-state, it is anticipated that the LEN approximation breaks down sooner, with the effects of an extended space charge becoming apparent at much lower voltages in the off-state. Electro-convective effects, which appear to set in as low as $-10 \mathrm{~V}$ in the side-mounted devices, can be expected to alter the current-voltage characteristics, even if large-scale mixing is suppressed. This may account for the appearance of the off-state transients in the NCM devices.

However, the absence of these off-state transients and the absence of the I-t inflection in the Nafion experiments indicate that EOF may play a substantial role in all of this, particularly in the off-state. Further experimental evidence, such as Nafion data for different degrees of asymmetry and/or Nafion data for a top-mounted device, could clarify this. Additional experiments which might clarify the situation are proposed in the following subsection.

A last, and perhaps less vexing issue is that the top-mounted NCM appears to reach a value for the limiting low-voltage rectification factor which is about twice the classical LEN value. The low-voltage limit was derived keeping only terms to leading order in the current density for the membrane concentration profile and also assuming the membrane could be treated as locally electro-neutral. The former assumption seems legitimate at first. However, based on the fact that expansions in terms of small currents for the voltage drops in the diffusion layers are asymmetric for asymmetric systems, one must wonder if including the first-order correction might improve this agreement. One of the chief (and only) results in the final chapter places the latter assumption on very shaky theoretical ground. 


\subsubsection{Future/continuing work}

In addition to conducting complimentary experiments using Nafion in a top-mounted device and in side-mounted devices with differing degrees of cross-sectional asymmetry which would greatly help to clarify the issues regarding the role of net EOF, several other suggestions for new experiments to compliment on-going theoretical work are suggested below.

\section{Low-voltage measurements, additional modeling/theory}

To test the 1D LEN predictions and determine to what degree this rectification mechanism manifests itself in an experimental system while minimizing the effects of extended space charge and EOF requires carefully performed low-voltage measurements. The current experimental set-up is more appropriate for measurements at high voltage and has a bias of about $2 \mathrm{~V}$, which makes accurate low-voltage measurements difficult if not impossible. Using the same device designs with a more appropriate power supply, for instance something used to make precision measurements in solid-state devices, with good stability and voltage steps in the $0.1 \mathrm{~V}$ or less range would be ideal.

However, a better test of the $1 \mathrm{D}$ model of chapter 2 does not even require a micronanofluidic device. In fact, there is nothing in the theory to suggest the length scales matter at all, apart from the requirement that there be a perm-selective element between two asymmetric regions. A geometrically asymmetric macro-fludic rectifier constructed from plexiglass cylinders and using a large piece of PC-NCM did not exhibit noticeable rectification, and the failure in this device may be attributable to a number of issues outside the scope of the simple theory. A more direct test would involve a very simple device such as that pictured in Figure 4.23, which could be milled from plastic. By employing flat electrodes, i.e. foil or a metallic sputter-coating on the bottoms of each of the milled plates, a 1D electric field can be guaranteed and the dimensions adjusted so that the diffusion layers fill the device completely in a reasonable amount of time. Similar to the experiments in Ref. [53], this device could very easily be filled with an electrolytic gel which would suppress electro-convective effects, known to suppress the overlimiting conductance, thus permitting greater latitude in 


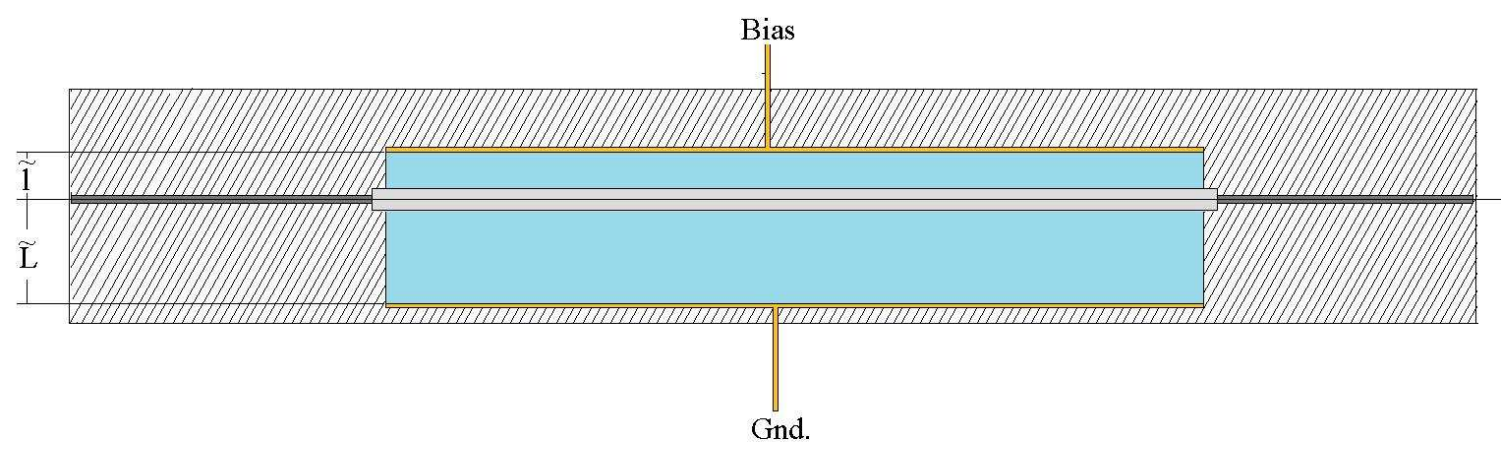

Figure 4.23: A simple experimental device to test the 1D steady-state theory.

the voltage range over which the experiments may be conducted. This would be advantageous as well in testing non-classical theories involving violations of local electroneutrality in both the electrolyte and membrane, such as will be presented in the next chapter.

\section{Device design modifications}

Many of the ideas regarding the growth of asymmetric diffusion layers and lengthselection processes would be best tested using a device design similar to that of Yossifon et. al. Instead of an array with asymmetric entrances between two reservoirs, a flat single nano-channel with asymmetric microchannels but no asymmetric overlap in the nanochannel entrances - such as pictured in Figure 4.24 would be ideal. This would permit imaging of diffusion layer growth and the DL length-selection phenomenon on both sides of the channel entrance and permit thorough investigations of the role of device asymmetry on diffusion layer growth as well as rectification to be carried out. Ideally, several such devices with different values of $\Gamma$ could be built and tested. It may also be interesting to consider various nanochannel depths to better understand the limitations of decreasing overlap on rectification. Such a design would also be advantageous for comparison with models, as both 1D models and fully 2D/3D models such as the COMSOL finite-element calculations, would be applicable to such systems. 


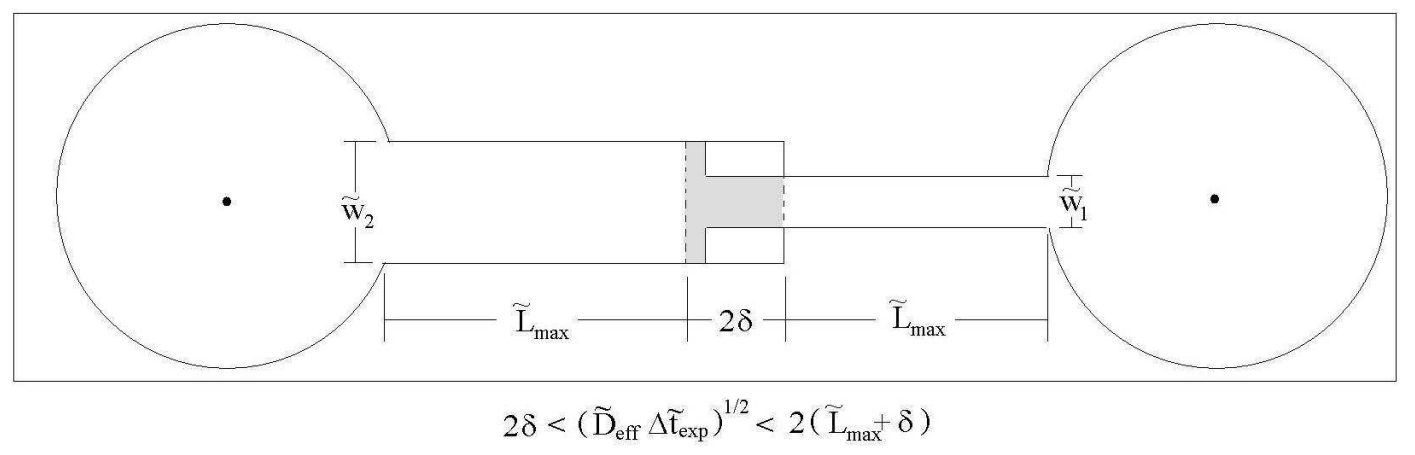

Figure 4.24: A device similar to the Yossifon et. al. design for investigating the relationship between geometry, time-dependent DL length asymmetry, and fluidic rectification. Note that the time intervals over which the experiments should be conducted are dependent upon device geometry. This is to ensure that the slow (sub-critical, electro-neutral) diffusion layers do not reach the electrodes so that the idea of a propagating diffuse front may be investigated. Presumably, these restrictions require modification for tests above the LEN threshold voltages, as per the theory in Ref. [49] 


\section{Chapter 5}

\section{Beyond the classical approximation}

\subsection{Chapter introduction and overview}

The model used to study rectification effects in Chapter 3 was based upon a quasi1D LEN theory that, strictly speaking, is only approximately valid for systems with very small Debye lengths at low voltages. In light of this, the apparent failure of the theory to capture quantitative details of a device that typically operates at high voltages is not surprising. Clearly, the theory should be extended to include higher voltages, i.e. violation of LEN and appearance of the macroscopic space charge, into the description. The systems in question may be more accurately represented by (at least) two dimensional models; this is especially relevant for obtaining a better picture of flux and field focusing effects, as well as understanding the growth of diffusion layers in asymmetric systems. Lastly, electro-osmotic and electro-convective effects should be included in the description. However, as with any particular effort, the question is not how to extend the theory to capture all of these effects, but rather which aspects of the behavior can be understood by a specific extension of the theory.

In section 5.2, the relationships between the governing effective diffusion length scale, and bulk concentration (i.e. intrinsic Debye length) for various estimated threshold voltages in the $Q 1 D L \cap Q E B$ theory of ideally permselective interfaces $[68,50,49]$ are used to compare overlimiting behavior of the data in chapter 4 and some of the experimental data of 
Yossifon et. al [66]. It is shown that estimates of this length scale increase for devices with increasing cross-sectional asymmetry. However, these estimates were derived for a somewhat different scenario, representative of a system with symmetric diffusion lengths and an ideally permselective membrane. As such, they must be interpreted cautiously. The effects of a nonnegligible co-ion flux through the membrane on voltage ranges corresponding to structural changes in the Q1DL are not yet known. Also, from chapter 3, it can be anticipated that the limiting currents in both off- and on- states are strongly influenced by the diffusion length in the microchannel. Because of these differences, it is not clear to which region of the device the estimated length scale corresponds.

Section 5.3 represents the first steps in the extension of the theory to the case of an ideally permselective membrane in an asymmetric system. Here it is shown that the range of validity of the electric field expansion outside of the membrane is larger than the expansion for the ideal membrane by a factor of the ratio of charge flux density to salt flux density. For weakly perm-selective systems this factor can be much greater than $O(1)$. It is also shown that the membrane interior exhibits charge separation on the order of the membrane width. A different aspect of the problem, related to the suspected mechanism for the onset of overlimiting conductance, is considered in section 5.4. There the ideal membrane approximation for thin non-equilibrium double-layers is employed to consider the linear stability of the quiescent electro-diffusion problem in a manner similar to Ref. [51]. However, the geometry studied here is similar to the off-state NCM/NMI device microchannel, where stability is considered for an electrolyte-membrane interface system confined in a narrow channel. It is shown that a minimum allowed wavenumber for perturbations exists, which may have interesting implications for the process of diffusion length selection.

\subsection{The effects of space charge at increasing fluxes and breakdown of the Q1DL description}

As is well-known and discussed in Chapter 2, as the voltage applied across the interface between a permselective solid and the flanking electrolyte layer(s) increases, the classical 
Table 5.1: Important threshold voltage estimates for concentrations $c_{o} \sim 1 \mathrm{mM}$ and $10 \mathrm{mM}$ giving Debye lengths of approximately $3.36 \mathrm{~nm}$ and $10 \mathrm{~nm}$ respectively. The numbers shown for the thresholds are voltages, i.e. the dimensionless orders $\times 0.0254 \mathrm{~V}$, i.e. the thermal voltage.

\begin{tabular}{|c|c|c|c|c|}
\hline $\mathrm{L}(\mathrm{m})$ & $\epsilon$ & $O(|\ln \epsilon|)$ & $O\left(\frac{4}{3}|\ln \epsilon|\right)$ & $O\left(\frac{1}{\epsilon|\ln \epsilon|^{3 / 2}}\right)$ \\
\hline \multicolumn{5}{|c|}{$c_{o} \sim 1 m M$} \\
\hline $10^{-2}$ & $1.37 \times 10^{-6}$ & $0.34 \mathrm{~V}$ & $0.45 \mathrm{~V}$ & $374 \mathrm{~V}$ \\
\hline $10^{-3}$ & $1.37 \times 10^{-5}$ & $0.28 \mathrm{~V}$ & $0.38 \mathrm{~V}$ & $49.5 \mathrm{~V}$ \\
\hline $10^{-4}$ & $1.37 \times 10^{-4}$ & $0.23 \mathrm{~V}$ & $0.30 \mathrm{~V}$ & $6.9 \mathrm{~V}$ \\
\hline \multicolumn{5}{|c|}{$c_{o} \sim 10 \mathrm{mM}$} \\
\hline $10^{-2}$ & $4.33 \times 10^{-7}$ & $0.37 \mathrm{~V}$ & $0.50 \mathrm{~V}$ & $1046 \mathrm{~V}$ \\
\hline $10^{-3}$ & $4.33 \times 10^{-6}$ & $0.31 \mathrm{~V}$ & $0.41 \mathrm{~V}$ & $135 \mathrm{~V}$ \\
\hline $10^{-4}$ & $4.33 \times 10^{-5}$ & $0.25 \mathrm{~V}$ & $0.34 \mathrm{~V}$ & $18.43 \mathrm{~V}$ \\
\hline
\end{tabular}

limiting current is approached then exceeded. This increase is accompanied by the appearance of an extended space charge region, which eventually renders quiescent electro-diffusive conduction, corresponding to the breakdown of the quasi-one-dimensional boundary layer approximation [50, 68, 51]. Employing Rubinstein and Zaltzman's various estimates and comparing with both the experiments in chapter 3 along with some of the experimental studies of Yossifon et. al. [66], it becomes clear that there are some un-resolved issues. While the ideal membrane estimates seem to provide some insight, particularly in relating the threshold voltage for applicability of the Q1DL formulation ([68]) to the onset of overlimiting conductance, they do not completely clarify the situation.

First, since it has been determined that field- and flux-focusing effects can obscure the limiting current window in perm-selective interfaces similar to the NCM/NMI [67], consider the experimental data of Yossifon et. al. [66]. For an electrolyte with bulk concentration 
Table 5.2: Governing effective diffusion length estimates for the asymmetric side-mounted PC-NCM devices assuming the maximum estimated voltage for the Q1DL corresponds to the I-t inflection.

\begin{tabular}{cccc}
\hline Channel depth $(\mu \mathrm{m})$ & $V_{\text {inf }}(\mathrm{V})$ & $\epsilon$ & $L_{\text {eff }}(\mathrm{m})$ \\
\hline 40 & 22 & $3.5 \times 10^{-5}$ & $2.9 \times 10^{-4}$ \\
30 & 24 & $3.2 \times 10^{-5}$ & $3.1 \times 10^{-4}$ \\
12 & 50 & $1.4 \times 10^{-5}$ & $7.1 \times 10^{-4}$ \\
\hline
\end{tabular}

$c_{o}=0.1 \mathrm{mM}\left(n_{o}=6.2 \times 10^{22} \mathrm{~m}^{-3}\right.$ and assuming a diffusion length equal to the distance to the electrode, i.e. $L=0.8 \mathrm{~mm}$, as in Ref. [66], the dimensionless Debye parameter is $\epsilon=1.32 \times 10^{-5}$. Using the estimate for the threshold zeta potential (the total voltage at the membrane-electrolyte interface, including the applied voltage and space-charge polarization) $O\left(1 / \epsilon|\ln \epsilon|^{3 / 2}\right)$ yields a threshold estimate of about $51 \mathrm{~V}$. The observed onset of overlimiting current is at about $8 \mathrm{~V}$. If instead the experimentally observed voltage is used to estimate the Debye parameter and corresponding diffusion length, the results are $\epsilon=1.16 \times 10^{-4}$ and $L=1.8 \times 10^{-4} \mathrm{~m}$.

There are several possible reasons for this discrepancy. One is that the diffusion length is in fact less than the distance to the electrode. If the width of the diffusion layer is estimated according to the scaling ${ }^{1} \Delta x=\sqrt{\mathcal{D} t}$ and the time between voltage intervals in the I-V experiments is $3 \mathrm{~s}$, then the diffusion length (for a KCL buffer with mobility $7.6 \times 10^{-8} \mathrm{~m}^{2} / \mathrm{s}$ ) is estimated to be $7.6 \times 10^{-5} \mathrm{~m}$, or about half the value obtained by equating the voltage threshold for overlimiting current with the Q1DL threshold of Zaltzman and Rubinstein. This value corresponds to an estimated voltage threshold of $6.8 \mathrm{~V}$. Recalling the comparison of the I-t and I-V behavior for the NCM devices in Chapter 4, it seems reasonable to suppose the actual diffusion length is more closely approximated by this estimate. Indeed, experimental work on a similar device verified the diffusive scaling holds approximately up to the point of vortex formation, wherein the length of the layer is selected by the instability [64]. In addition to the experiments suggested in the previous chapter, this could be investigated

\footnotetext{
${ }^{1}$ This is not strictly valid!
} 
by considering the I-t behavior for the nanoslot device and looking for similar responses to those seen in the NCM devices, i.e. the starting increase of the I-t slope and the inflection.

This correspondence, as well as the estimates in Table 3.1, make a compelling case for associating the Q1DL break-down with the onset of overlimiting conductance in systems without strong field/flux focusing, and strongly suggest that this criterion may also be associated with the I-t inflections in the flux/field focusing NCM/NMI. However, if that is the case, it is clear, at least for strongly perm-selective membranes, that this voltage threshold is many orders of magnitude higher than the critical knee voltage for the classical limiting current.

From an examination of the data in Table 5.1, the murkiness of the situation becomes more apparent; these numbers seem to justify some of the ideas in chapter 2 and 3 regarding a connection between increasing asymmetry and increasing effective diffusion length scale, but the correspondence is not clear. To make matters more confounding, a consideration of the same data for the top-mounted NCM device reveals an effective diffusion length scale that is about two orders of magnitude larger than those for the side-mounted NCM devices. This is completely inconsistent with the whole picture, unless something not included in any of the fundamental theoretical descriptions considered thus far, such as net EOF, can explain the discrepancy. As noted in the previous chapter, strong net EOF is suspected to play an important role in the modification of diffusion layers in the NCM, resulting in some loss of rectification strength as well as an accompanying shift in the values of these thresholds (in as much as they are influenced by this effective diffusive length scale.)

Since in both the NCM and the devices used by Yossifon et. al., there are a significant number of co-ions passing through the channel(s) as well as possible EOF, particularly in the NCM, it may be useful to attempt a similar 'back of the envelope' estimate for the sidemounted Nafion device. Recall that the Nafion I-t behavior lacked a conspicuous inflection. However, the I-t slope undergoes a marked increase around $600 \mathrm{~V}$, so this point is chosen as an estimate for the onset of overlimiting conductance and yields the estimates $\epsilon=8.05 \times 10^{-7}$ and $L_{e f f}=0.012 \mathrm{~m}$. While it is known that the picture used in Hunter's derivation [?] of the diffusion layer scaling $\sqrt{D t}$ is not strictly valid for systems above the limiting current [49], experimental evidence [64] suggests that it gives a reasonable order-of-magnitude estimate. 
For the phosphate buffer, using the diffusivity of the sodium ion and the total measurement time of 300s, the diffusive thickness is about $600 \mu \mathrm{m}$, about half the value for the effective length scale obtained from the Q1DL threshold estimate. If this can be taken for granted, then it would seem that the lower permselectivity and non-negligible net EOF present in the NCM/NMI systems must be playing an important role. Of course, in addition to the fundamentally different character of the governing equations in the electro-diffusional free boundary problem, once Rubinstein's vortices develop, they will determine the length scale by a non-linear wavenumber selection process which is not yet fully understood [64, 66]. Since the on-state side of the membranes in the side- and top- mounted devices in chapter 3 are not accessible to imaging, it is not possible to determine when the vortices develop and how they behave in either the NCM/NMI or the Nafion devices.

Given the number of possible mechanisms contributing to the stirring and diffusionallength scales $[68,5,67]$, it is difficult to make any conclusions whatsoever, other than perhaps that the understanding of the problem, particularly for the case of nanochannels, is at this point unclear. The next two sections of this chapter aim to establish some basic results which may be used in the course of developing a theory of non-equilibrium electro-osmotic slip for non-ideal permselective membrane interfaces in geometrically asymmetric systems. These along with a re-visitation of the diffusive scaling problem, but from the point of view provided by the results in Ref. [49] relevant to situations above the limiting current, may eventually resolve this confusion.

\subsection{Finite- $\epsilon$ formulation of problem for non-ideal mem- branes}

The following derivation follows that in Ref [68] closely. However, there are some inherent differences between the two scenarios which complicate the analysis. In this case, the fields and concentrations inside the membrane itself must be taken into account, and at the least the voltage and concentration at the right membrane boundary must be specified. As indicated 


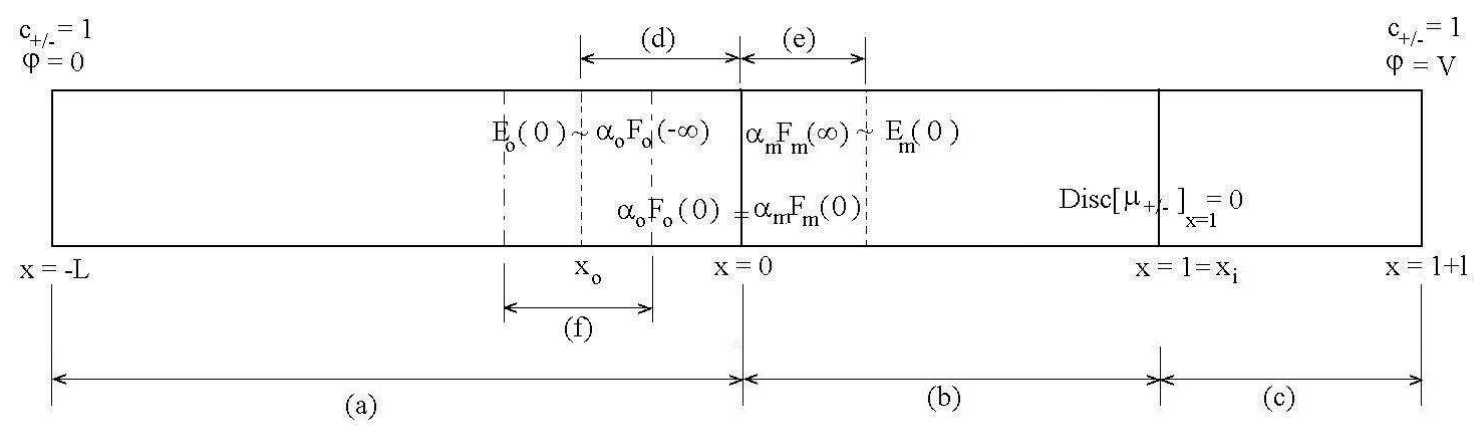

Figure 5.1: 1D geometry showing matching, boundary, and jump conditions for the singularly perturbed electro-diffusive problem at an interface with a non-ideal membrane. The regions shown are: (a) and (c), the left and right electrolyte layers respectively, i.e. assumed to be quasi-electroneutral bulk (QEB) (b) the membrane interior, (d) the inner region up to the extended space charge outside the membrane, and (e) the inner region inside the membrane. (f) is the $Q 1 D L \cap Q E B$ overlap region. Note that the point $x_{o}$ is shown to correspond to the outer edge of the extended space charge here, and that this picture is valid then for currents above the classical limiting value. The point $x_{i}$ is assumed to be at the right membrane-solution boundary, however, this picture may need revision following a rigorous and complete boundary layer analysis

in Figure 5.1, the right boundary conditions may also be assumed to be approximated by the LEN voltage drops and continuity of electrochemical potentials at this interface. This is only because the situation envisioned for study involves a large negative bias at the far right end, so that the depleted layer forms in the left side of the system and the right side maintains quasi-local electroneutrality (see any of the references $[68,50,49]$.) The point $x_{o}$ is the extrapolated zero of the linear concentration profile in the left region, and may be greater than or less than zero, depending on the magnitude of the applied voltage (i.e. upon the current density.) However, in the current analysis, no such distinctive interpretation of $x_{i}$ clearly emerges, so it will be left as arbitrary, and may be taken as the right membrane boundary for convenience if need be.

In what follows, the first crucial steps in the complete boundary layer analysis are laid out and may be compared to the steps and analogous results in the references. Recalling the 1D electro-diffusion equations from chapter 3

$$
c_{ \pm}^{\prime} \pm c_{ \pm} \varphi^{\prime}=-\frac{j_{ \pm}}{\mathcal{D}}
$$


may be written in terms of the scaled field, $E=-\epsilon \varphi^{\prime}$, obtaining the equations

$$
\mathcal{D}\left[\left(c_{+}^{\prime}-c_{-}^{\prime}\right)-\left(c_{+}+c_{-}\right) \frac{E}{\epsilon}\right]=-2 J_{-}
$$

and

$$
\mathcal{D}\left[\left(c_{+}^{\prime}+c_{-}^{\prime}\right)+\left(c_{-}-c_{+}\right) \frac{E}{\epsilon}\right]=-2 J_{+}
$$

The Poisson equation may be re-written as

$$
c_{-}=c_{+}-\epsilon E^{\prime}-N(x)
$$

and differentiated

$$
c_{+}^{\prime}-c_{-}^{\prime}=\epsilon E^{\prime \prime}+N^{\prime}(x)
$$

Re-writing the cation electro-diffusion equation,

$$
\frac{c_{+} E}{\epsilon}=c_{+}^{\prime}+\frac{j_{+}}{\mathcal{D}}
$$

and using the Poisson equation allows the following expressions to be written for the concentration profiles outside and inside of the membrane. The solution layers outside the membrane have

$$
\epsilon E^{\prime \prime}-2 c_{+}^{\prime}+E^{\prime} E=2 J_{+}
$$

which can be integrated,

$$
c_{+}=\frac{\epsilon E^{\prime}}{2}+\frac{E^{2}}{4}-J_{+}\left(x_{o}-x\right)
$$

for the concentration profile outside the membrane. Notice that the leading order concentration profile is essentially the same as the LEN solution, taking the limit $\epsilon \rightarrow 0$. Inside the membrane, for an arbitrary 1D fixed charge density $N(x)$,

$$
\epsilon E^{\prime \prime}+N^{\prime}(x)-2 c_{+}^{\prime}+E^{\prime} E+\frac{N E}{\epsilon}=\frac{2 J_{+}}{\mathcal{D}}
$$

Or, re-arranging and integrating,

$$
c_{+}=\frac{\epsilon E^{\prime}}{2}+\frac{E^{2}}{4}-\frac{J_{+}}{\mathcal{D}}\left(x-x_{i}\right)+\frac{1}{2 \epsilon} \int_{x_{i}}^{x} N(y) E(y) d y+\frac{N}{2}
$$

in the membrane interior. Note that $x_{o}$ and $x_{i}$ are suitably defined integration constants. The value of $x_{o}$ can be interpreted the same way as in [68], as the point at which the extrapolated LEN concentration profile would go zero for low voltages, or equivalently for high 
voltages, the outer edge of the extended space charge. As indicated in ??, the inner scaled solutions $F$ can be equated at the membrane-electrolyte boundary and the inner and outer solutions connected through asymptotic matching procedures.

Substituting the above solutions back into equation 5.3 yields the equations for the scaled E-fields in each region. The concentration profile inside the membrane includes contributions from both the fixed charge density and the integral over the E-field, and there is no a priori guarantee that local electro-neutrality holds over any region inside the membrane. Determining whether or not such a condition arises naturally from the governing equations for finite- $\epsilon$ is one of the goals in this section.

\subsubsection{The electrolyte layers outside the membrane}

For the two regions on either side of the membrane containing the solution layers, the fields are governed by an equation similar to that for the ideal membrane case ([68])

$$
\epsilon^{2} E^{\prime \prime}=\frac{1}{2} E^{3}-2 E J_{+}\left(x_{o}-x\right)-2 \epsilon G J_{+}
$$

Hence the analysis of the problem outside the membrane is similar to that in the reference. However, just as the LEN non-ideal membrane case involved somewhat more convoluted computation than the ideal case, so will the non-ideal case for finite $\epsilon$. The chief complication arises in the boundary conditions themselves. Since the co-ion current is no longer suppressed, the co-ion electrochemical potential cannot be taken to be everywhere equal to zero. However, it is still true. Here it is assumed that the ratio of charge flux density to salt flux density, $G=J_{-} / J_{+}$, is known, and it is further assumed that the potential at the membrane-electrolyte interface may be found from the solution of the full problem. For now, it is just taken as the value $V_{m}$. The concentrations may be assigned as

$$
c_{+}(0)=p_{1}
$$

and

$$
c_{-}(0)=p_{2}
$$


Assuming a solution of the form

$$
E(x)=\sum_{n} \epsilon^{n} E_{n}(x)
$$

and substituting this into equation 5.3.1 above,

$$
\sum_{n} \epsilon^{n+2} E_{n}^{\prime \prime}=\frac{1}{2} \sum_{l, m, n} \epsilon^{l+m+n} E_{l} E_{m} E_{n}-2 J_{+}\left(x_{o}-x\right) \sum_{n} \epsilon^{n} E_{n}-2 \epsilon G J_{+}
$$

\section{The 0 -th order solutions}

gives the following leading-order equation for the outer solution,

$$
\frac{1}{2} E_{o}^{3}-2 J_{+} E_{o}\left(x_{o}-x\right)=0
$$

or,

$$
E_{o}\left[\frac{1}{2} E_{o}^{2}-2 J_{+}\left(x_{o}-x\right)\right]=0
$$

This equation yields the roots,

$$
E_{o}=0
$$

and

$$
E_{o}= \pm 2 \sqrt{J_{+}\left(x_{o}-x\right)}
$$

This solution will be applied to the region $x \leq 0$ since the region $1 \leq x \leq 1+\ell$ is assumed to be well-described by the LEN solution, ignoring the double-layers on this side and restricting attention to the case where $V<0$.

\section{Higher-order corrections for the root $E_{o}=0$}

For the case $E_{o}=0$ since $l+m+n=1$ requires two of $l, m, n$ to be zero, there is a factor $E_{o}^{2}$ in the product $E_{l} E_{m} E_{n}$ so that the $E^{3}$ term is zero and the first-order correction for this root is

$$
E_{1}=-\frac{G}{\left(x_{o}-x\right)}
$$

The second order correction is given by

$$
-2 J_{+}\left(x_{o}-x\right) E_{2}=0
$$


or $E_{2}=0$.

The third order correction is given by

$$
E_{1}^{\prime \prime}=\frac{1}{2} E_{1}^{3}-2 J_{+}\left(x_{o}-x\right) E_{3}
$$

or

$$
E_{3}=-\frac{4 G+G^{3}}{4 J_{+}\left(x_{o}-x\right)^{4}}
$$

\section{Higher-order corrections for the non-zero root}

For the second set of leading-order roots, the first-order correction is given by

$$
\frac{3}{2} E_{o}^{2} E_{1}-2 J_{+}\left(x_{o}-x\right) E_{1}-2 J_{+} G=0
$$

or,

$$
E_{1}=\frac{G}{2\left(x_{o}-x\right)}
$$

The second order correction for these roots is given by

$$
E_{o}^{\prime \prime}=\frac{3}{2}\left[E_{1}^{2} E_{o}+E_{o}^{2} E_{2}\right]-2 J_{+}\left(x_{o}-x\right) E_{2}
$$

which may be re-arranged and solved for $E_{2}$,

$$
E_{2}=\mp \frac{1}{8 J_{+}^{1 / 2}\left(x_{o}-x\right)^{5 / 2}}\left(1+\frac{3}{2} G^{2}\right)
$$

Outer series solution for electric field exterior to the membrane

$E(x)= \begin{cases}-\epsilon \frac{G}{\left(x_{o}-x\right)}-\epsilon^{3} \frac{4 G+G^{3}}{4 J_{+}\left(x_{o}-x\right)^{4}}+\ldots & :\left(x_{o}-x\right) \gg O\left(\epsilon^{2 / 3}\right) \\ \pm 2 \sqrt{J_{+}\left(x_{o}-x\right)}+\epsilon \frac{G}{2\left(x_{o}-x\right)} \mp \frac{\epsilon^{2}}{8 \sqrt{J_{+}}\left(x_{o}-x\right)^{5 / 2}}\left(1+\frac{3}{2} G^{2}\right)+\ldots & :\left(x_{o}-x\right) \ll O\left(\epsilon^{2 / 3}\right)\end{cases}$

The range of validity can be established by considering for what order of $\left(x_{o}-x\right)$ do the terms corresponding to higher-order corrections in powers of $\epsilon$ approach the same magnitude as the leading order term. For sufficiently perm-selective systems, $G=O(1)$ and this is the same order as in the ideal problem, so that the following may be assumed to hold as well,

$$
y<O(1 /|\ln \epsilon|)
$$




$$
\left|c_{+}-c_{-}\right| \ll O\left(\epsilon^{2 / 3}\right)
$$

and

$$
\left|\varphi^{\prime}\right| \leq O\left(\epsilon^{-2 / 3}\right)
$$

However, this correspondence cannot be taken for granted. Consider the definition of $G$ in terms of the selectivity $\beta=\left|j_{+} / j_{-}\right|$,

$$
G=\frac{\beta+1}{\beta-1}
$$

Clearly, for highly perm-selective systems, the solution approaches that of the ideal system and the analysis in Ref [68] may be applied. However, the pole at $\beta=1$ suggests that systems like the NCM and nanochannel devices can have ranges, particularly at higher concentrations, wherein the selectivity function $G$ can be quite large. In such cases, by inspection of the expansion in 5.3.1, the range of validity shifts by a factor of $G$. Presumably this will affect all of the estimates. The results in this section are a first step in carrying out this analysis.

Integration of equation 5.3.1 across the QEB and up to the outer edge of the Q1DL yields (to leading order in $\epsilon$ ),

$$
\varphi(x)=-G \ln \left(x_{o}-x\right)+\Phi=\bar{\varphi}
$$

(where the overbar denotes quantities in the $Q 1 D L \bigcap Q E B$.) While it is no longer the case that the co-ion electrochemical potential is still everywhere zero, it is true that both electrochemical potentials are continuous at the QEB/Q1DL boundary. Thus the relationship between $\Phi$ and the anion electrochemical potential appearing in equation 3.13 of Ref [68] does not hold here. However, the relationship, $\Phi=\phi(0)$ still holds, where $\phi$ is the potential in the QEB region. Also note that, for the same layer thickness $\left(x_{o}-x\right)$ the potential drop for non-ideal systems is larger by a factor of $G$ than in an ideal system with comparably thick layers. Since this thickness is considered a control parameter in the changing morphology of the layer and impending instability (again, see [68]), this seems to suggest non-ideal systems may in fact tolerate higher voltages. However, this is by no means a rigorous conclusion as much as it is a suggestive idea. 
Jarrod E. Schiffbauer

\section{Re-scaling and the inner solution}

To study the inner solution to the b.v.p. in the regions exterior to the membrane, a new scaling must be introduced. Since the problem is singular, with the second derivative being multiplied by $\epsilon^{2}$ in the equation governing the fields, a proper re-scaling would be set this coefficient to have a magnitude $O(1)$. To do this, assume a transformation

$$
E=\chi F
$$

and

$$
z=\xi x
$$

Now, multiplying equation 5.3 .1 by $\mathcal{D} / J_{+} \epsilon$ and transforming,

$$
\frac{\mathcal{D}}{J_{+}} \chi \xi^{2} \epsilon F^{\prime \prime}=\frac{\mathcal{D} \chi^{3}}{2 J_{+} \epsilon} F^{3}-\frac{2 \mathcal{D} \chi}{\epsilon \xi}\left(z_{o}-z\right) F-2 G
$$

By demanding the coefficient of the second derivative be $O(1)$,

$$
\chi=\frac{J_{+}}{\mathcal{D} \epsilon \xi^{2}}
$$

For the coefficient of the linear term to be proportional $z o-z$, we require that $2 \mathcal{D} \chi=\epsilon \xi$ and obtain

$$
\xi=\left(2 J_{+}\right)^{1 / 3} \epsilon^{-2 / 3}
$$

and

$$
\chi=\frac{\left(J_{+} \epsilon\right)^{1 / 3}}{2^{2 / 3} \mathcal{D}}
$$

and the following form of the inhomogeneous Painleve equation of the second kind is obtained,

$$
F^{\prime \prime}=\frac{1}{8 \mathcal{D}^{2}} F^{3}-\left(z_{o}-z\right) F-2 G \mathcal{D}
$$

By transforming equation 5.3 to the inner variables and evaluating at $z=0$, the following boundary condition is obtained,

$$
\left(F^{\prime}+\frac{1}{8 D^{2}} F^{2}\right)_{z=-\chi}=\frac{2^{1 / 3} \mathcal{D}}{\left(J_{+} \epsilon\right)^{2 / 3}} p_{1}+\mathcal{D} z_{o}
$$

Again, as in the references, the left side of the equation is bounded, but the right side unbounded for $\epsilon \rightarrow 0$, and by the same reasoning, implies the existence of the boundary layer. 
For very large $z$, with $|F| \leq O(1)$ from the bounding condition on $\left|\varphi^{\prime}\right|$, the powers of the field and its derivatives are bounded (since it is assumed that electroneutrality is approached as $z \rightarrow-\infty$ ) the following asymptotic expression for $F$ may be written,

$$
F_{\infty}(z)=-\frac{2 \mathcal{D} G}{z_{o}-z}
$$

The reduced zeta potential is defined as

$$
\zeta=-V_{m}-\Phi
$$

such that this represents the drop from the membrane across the entire Q1DL and $\Phi$ is the regular part of the electric potential, i.e. the integration constant appearing in eqn. 5.3.1. The value of the potential at the membrane-electrolyte interface, $-V_{m}$, may be thought of as the difference between the potential drop across the $Q 1 D L \cap Q E B$ and the drop across the (appropriate composition at a given voltage) for the inner problem,

$$
\varphi(x)-\chi \int_{z}^{0} F\left(z^{\prime}\right) d z^{\prime}=-V_{m}
$$

This may be re-written in a form similar to eqn. 3.18 of Ref. [68],

$$
-G \ln \left(x_{o}-x\right)-\chi \int_{z}^{0} F\left(z^{\prime}\right) d z^{\prime}=-V_{m}-\Phi
$$

\subsubsection{The membrane interior}

By following the same procedure inside the membrane, a more general form of the above equation (allowing for the presence of fixed charges) is obtained using equation 5.3,

$$
\frac{1}{2} \epsilon E^{\prime \prime}=\frac{E}{2 \epsilon^{2}} \int_{x_{i}}^{x} N\left(x^{\prime}\right) E\left(x^{\prime}\right) d x^{\prime}+\frac{1}{4 \epsilon} E^{3}-\frac{E}{\epsilon}\left[\frac{J_{+}}{\mathcal{D}}\left(x-x_{i}\right)-\frac{N}{2}\right]-\frac{G J_{+}}{\mathcal{D}}
$$

For the present purpose, it will suffice to assume that $N$ is uniform throughout the membrane except for a very thin layer, at least as thin or thinner than a Debye layer. It is not clear a priori whether this is physically realistic, especially in the case of NCM and other nanochannel devices where the surface charge may be a function of the bulk concentration $[54,57]$. For a numerical solution of the full problem, it may be advantageous to consider $N$ to be some step-like function of position with a smooth but rapid decay at the membrane edges. 
Here, the question being addressed is whether or not the solution of this equation implies local electro-neutrality inside a model 1D membrane on scales of the order of the membrane width, so this issue is ignored for the time-being.

To do this, consider a perturbation approach for the solution of the scaled field, i.e. by assuming a solution of the form

$$
E=\sum_{n} \epsilon^{n+1} E_{n}
$$

and substituting the series into the governing equation

Proceeding term-by-term, the following approximations are obtained for each of the four terms that involve $E$,

$$
\begin{aligned}
\frac{1}{2} \epsilon E^{\prime \prime} & =\frac{1}{2} \sum_{n} \epsilon^{n+2} E_{n}^{\prime \prime} \\
\frac{E}{2 \epsilon^{2}} \int_{x_{i}}^{x} N E d x^{\prime} & =\frac{1}{2} \sum_{n, m} \epsilon^{n+m} E_{n} \int_{x_{i}}^{x} N E_{m} d x^{\prime} \\
\frac{1}{4 \epsilon} E^{3} & =\frac{1}{4} \sum_{n, m, \ell} \epsilon^{n+m+\ell+2} E_{n} E_{m} E_{\ell} \\
\frac{J_{+} E}{\epsilon \mathcal{D}}\left(x-x_{i}\right) & =\frac{J_{+}\left(x-x_{i}\right)}{\mathcal{D}} \sum_{n} \epsilon^{n} E_{n} \\
\frac{N E}{2 \epsilon} & =\sum_{n} \epsilon^{n} E_{n}
\end{aligned}
$$

Since this is a singular problem, we anticipate that the leading-order $(\epsilon=0)$ expansion yields an outer solution for the problem. The inner solution can then be found by a re-scaling of the variable, and a quick examination of dominant balance (or comparison by analogy to the typical EDL boundary-layer problem) suggests that the appropriate scaling is

$$
z=x / \epsilon
$$

This will be considered shortly. However, the outer solution is itself useful and may be obtained as follows:

The leading-order outer problem is given by the solution of

$$
\frac{E_{0}}{2} \int_{x_{i}}^{x} N E_{0} d x^{\prime}=\frac{N}{2}+\frac{J_{+}}{\mathcal{D}}\left(x-x_{i}\right)-\frac{G J_{+}}{E_{0}}
$$


Differentiating and re-arranging the above equation one obtains,

$$
E_{0}^{\prime}=\frac{E_{0}^{2}}{G}\left(\frac{N E_{0}}{J_{+}}-\frac{N^{\prime}}{2 J_{+}}-\frac{1}{\mathcal{D}}\right)
$$

Since attention is being restricted to regions in the membrane where the charge density is constant, this equation may be separated and integrated, neglecting the fixed charge density gradient,

$$
\int \frac{d E_{0}}{E_{0}^{2}\left(\frac{N E_{0}}{J_{+}}-\frac{1}{\mathcal{D}}\right)}=\frac{x-x_{i}}{G}
$$

to yield

$$
\frac{1}{E_{0}(x)}-\frac{1}{E_{0}\left(x_{i}\right)}+\frac{\mathcal{D} N}{J_{+}} \ln \left[\frac{E_{0}\left(x_{i}\right)\left(\mathcal{D} N E_{0}(x)-J_{+}\right)}{E_{0}(x)\left(\mathcal{D} N E_{0}\left(x_{i}\right)-J_{+}\right)}\right]=\frac{x-x_{i}}{G \mathcal{D}}
$$

Notice that this is very similar to equation 3.4 .5 in chapter 3 for the non-ideal membrane concentration profile but does not yield the locally electroneutral profile there. However, as will be seen shortly, the limit $G \rightarrow \infty$ implies an approach to electro-neutrality, as does the approach to equilibrium, ie. $J_{+} \rightarrow 0$.

For any region inside the membrane, Gauss's law gives,

$$
\oint \overrightarrow{\tilde{E}} \cdot \overrightarrow{d A}=\frac{q_{\text {enc }}}{\varepsilon \varepsilon_{0}}
$$

For the 1D case, considering the membrane to be an infinite plane, where the field is defined $\tilde{E}=-\varphi^{\prime}$ we may use the pillbox construction for the Gaussian surface, with one end located at $x=x_{i}$ and the other at some arbitrary point $x$. By symmetry considerations, it is clear only the field fluxes through the ends of the pillbox contribute, or, per unit area of an infinite 1D membrane,

$$
\tilde{E}(x)-\tilde{E}\left(x_{i}\right)=\frac{q_{\mathrm{enc}}}{\varepsilon \varepsilon_{0}}
$$

Thus for a membrane interior to obey local electro-neutrality on a scale comparable to the membrane width the inverse field terms must cancel and the numerator and denominator appearing the logarithmic term must be equal and the logarithm must equal zero in the locally electro-neutral membrane as well. However, note that the right-hand-side does not vanish over any finite interval; the implication is that a membrane of finite, non-zero thickness cannot be locally electro-neutral between any two points in the outer region. The leading-order 
contribution to the field $\tilde{E}$ is $E_{0}$, so that even in the limit $\epsilon \rightarrow 0$ the membrane does not obey local electro-neutrality. However, it is possible that the compensating charge is contained in one or more of the boundary layers, either inside or in the solution adjacent to the membrane. The present results bear no conclusions regarding this, so to proceed further, the full problem must be solved.

\section{Inner problem for membrane interior}

As a final starting piece, let's attempt to re-scale the problem according to the following general scheme:

$$
E=\epsilon F
$$

and

$$
\begin{gathered}
z=\frac{x}{\epsilon} \\
\frac{1}{2} F^{\prime \prime}=\frac{\epsilon}{2} F \int_{z_{i}}^{z} N F d z^{\prime}+\frac{\epsilon^{2}}{4} F^{3}-F\left[\frac{J_{+} \epsilon}{\mathcal{D}}\left(z-z_{i}\right)-\frac{N}{2}\right]-\frac{G J_{+}}{\mathcal{D}}
\end{gathered}
$$

Using the asymptotic expansion for $F$,

$$
F=\sum \epsilon^{n} F_{n}
$$

we obtain the following inner problem for the inside of the membrane,

$$
\frac{1}{2} F_{0}^{\prime \prime}=\frac{F_{0} N}{2}-\frac{G J_{+}}{\mathcal{D}}
$$

For $\left.\mid F_{0}(z)\right) \mid$ bounded as $z \rightarrow \infty$, the solution is given by

$$
F_{0}(z)=c \exp (-N z)+\frac{2 G J_{+}}{\mathcal{D} N}
$$

Then, looking towards matching this with the leading-order outer solution inside the membrane, in the limit $z \rightarrow \infty$,

$$
F_{0}(z \rightarrow \infty)=\frac{2 G J_{+}}{\mathcal{D} N}
$$

From the requirements of matching conditions, this must also be the limit for $E_{0}$ as $x \rightarrow 0$, however since it is not possible to re-arrange the leading order outer solution to provide a 
convenient expression for $E_{0}(x)$, the full composite expansion will not be pursued any further at this point. The complete analysis of the possible dual-boundary layers at the membranesolution interface is a topic best left for future work.

\subsection{Electro-osmotic instability of a confined electrolyte due to non-equilibrium electro-osmotic slip}

The goal of this calculation is to extend previous studies on electrokinetic instability $([50,51,68]$, to include the effects of confinement in a narrow channel. The most basic version ([51]) of the electrokinetic model is employed for this purpose, i.e. the LEN approximation is assumed valid except in thin boundary layers where a non-equilibrium slip velocity allows the inclusion of the de-stabilizing effects of the developed non-equilibrium space charge. The results herein may be compared to those in the references for the laterally unbounded $(x \rightarrow$ $\pm \infty)$ case. Variations on this theme are being considered. In particular, upon completing the analysis in the previous section, it would be interesting to see how the linear stability of the solution compares between ideal and non-ideal membranes. The complete analysis would permit more accurate estimates of the threshold voltages for such systems and hopefully clarify some of the issues raised in the first section of this chapter.

\section{Geometry and boundary value problem}

Consider the 2D LEN boundary value problem for the geometry shown in the figure. The two side-walls at $x= \pm h / 2$ have no-slip and no-flux boundary conditions and interact only through viscous dissipation. Thus for a symmetric, binary electrolyte, the side walls constitute an impenetrable boundary which does not affect the $x$-dependence of the unperturbed solution (i.e. the Q1DL solution.) The cation-selective membrane surface is at $y=0$ and the outer edge of the EDL is located at $y=y_{0}$, where a non-equilibrium electroosmotic slip condition for an ideal membrane is assumed. At $y=1$, there is a boundary with fixed electrochemical potentials and a no-slip condition. The governing equations for 


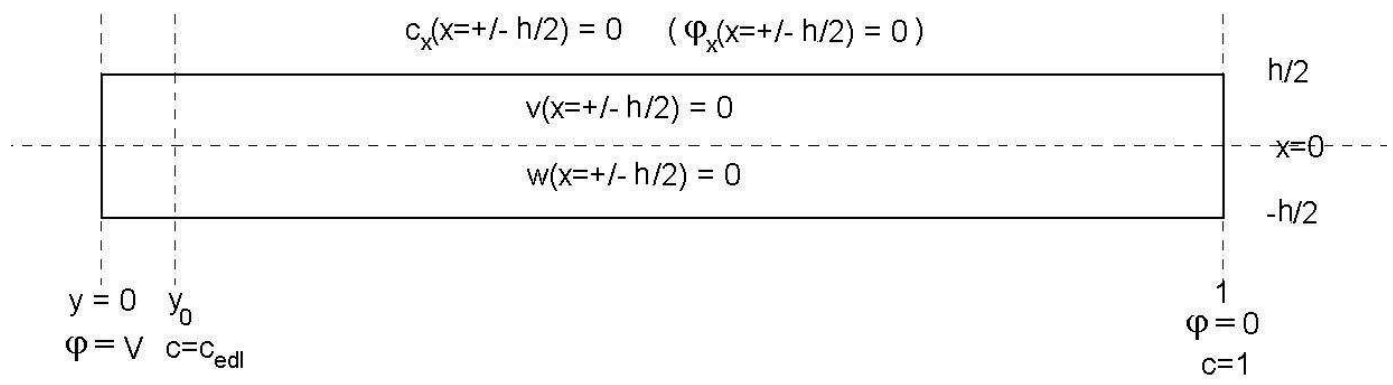

Figure 5.2: 2D channel geometry and boundary conditions for the stability problem. Notice the membrane/non-equilibrium double-layer are located at the origin, left-side of the figure, with the positive y-direction along the channel. No slip and no flux boundary conditions are imposed on the walls and axial symmetry is assumed.

this version of the problem are that representing the outer part of a singularly perturbed problem (i.e. as in [51] and references therein):

Assuming (approximate) stoichiometric electro-neutrality in the everywhere except boundary layers, the neutral salt density is governed by

$$
c_{t}+P e \vec{v} \cdot \nabla c=\triangle c
$$

subject to the boundary conditions, at $y=y_{0}$,

$$
c\left(x, y_{0}\right)=a\left(\varepsilon c_{y}\left(x, y_{0}\right)\right)^{2 / 3}
$$

at $y=1$,

$$
c(x, 1)=1
$$

and also on the walls, at $x= \pm h / 2$,

$$
c_{x}\left( \pm \frac{h}{2}, y\right)=0
$$

The constant $a$ is related to the boundary value of the inner scaled field $F$ and its first derivative and is of order 1 . The hydrodynamic equations are the low-Reynolds' number Navier-Stokes equation

$$
\frac{1}{S c} \vec{v}_{t}=-\nabla p+\triangle \vec{v}
$$

and the condition for incompressibility

$$
\nabla \cdot \vec{v}=0
$$


The Navier-Stokes equation may be re-written in terms of the velocity $\vec{v}=u \hat{i}+w \hat{j}$ by taking the curl, since $\nabla \times \nabla p=0$, and making use of the incompressibility condition above along with the identity

$$
\triangle \vec{A}=\nabla(\nabla \cdot \vec{A})-\nabla \times \nabla \times \vec{A}
$$

for any vector $\vec{A}$.

$$
\frac{1}{S c} \nabla \times \vec{v}_{t}=-\nabla \times \nabla \times \nabla \times \vec{v}
$$

There are 2 boundary conditions on each component of the fluid velocity as follows: at $y=y_{0}$,

$$
w\left(x, y_{0}\right)=0
$$

and the non-equilibrium slip condition,

$$
u\left(x, y_{0}\right)=-\frac{1}{8} V^{2}\left(\frac{c_{x y}\left(x, y_{0}\right)}{c_{y}\left(x, y_{0}\right)}\right)
$$

at $y=1$, a no-flux condition,

$$
w(x, 1)=0
$$

and a no-slip condition

$$
u(x, 1)=0
$$

\section{General form of linearized equations}

Assuming the perturbations may be written

$$
c=c_{0}+\delta e^{\lambda t} c_{1}(x, y)
$$

and

$$
\vec{v}=\vec{v}_{0}+\delta e^{\lambda t} \vec{v}_{1}(x, y)
$$

the governing equations may be written to $O(\delta)$ as

$$
\lambda c_{1}(x, y)+\operatorname{Pe}\left(\vec{v}_{0} \cdot \nabla c_{1}(x, y)+\vec{v}_{1} \cdot \nabla c_{0}\right)=\triangle c_{1}(x, y)
$$

for the Q1DL solution at the limiting current density, $c_{0}=y$ and $\vec{v}_{0}=0$, this becomes

$$
\lambda c_{1}(x, y)+P e w_{1}(x, y)=\triangle c_{1}(x, y)
$$


Upon carrying out the curls in equation 5.4, operating on the result with $\partial / \partial x$ and using the incompressibility condition, the fluid velocity perturbations are governed by the following equation,

$$
\frac{\lambda}{S c}\left(w_{1 x x}+w_{1 y y}\right)=2 w_{1 x x y y}+w_{1 x x x x}+w_{1 y y y y}
$$

\section{Linearized boundary conditions}

Following the calculations in the references, the position of the outer edge of the EDL/SCL, $y_{0}$, is considered to be similarly perturbed, i.e.,

$$
y_{0}=0+\delta e^{\lambda t} y_{1}(x)
$$

The value of the salt concentration at this boundary, perturbing about the Q1DL solution, is (keeping terms to leading order in $\delta$ ),

$$
c\left(x, y_{0}\right)=\delta e^{\lambda t}\left[y_{1}(x)+c_{1}(x, 0)\right]
$$

In this case $c_{y}=1+\delta e^{\lambda t} c_{1 y}(x, 0)$, so the following give expressions for fractional powers of this derivative to first order,

$$
c_{y}\left(x, y_{0}\right)^{2 / 3} \approx 1+\frac{2}{3} \delta e^{\lambda t} c_{1 y}(x, 0)
$$

and

$$
c_{y}\left(x, y_{0}\right)^{-1 / 3} \approx 1-\frac{1}{3} \delta e^{\lambda t} c_{1 y}(x, 0)
$$

Using these and equation (13) from [51], one obtains

$$
y_{1}(x)=-\frac{1}{3}\left(\frac{3}{4} \varepsilon V\right)^{2 / 3} c_{1 y}(x, 0)
$$

Putting this together with the boundary conditions and collecting terms of $O(\delta)$,

$$
c_{1}(x, 0)=\left[\frac{2}{3} a_{0} \varepsilon^{2 / 3}+\frac{1}{3}\left(\frac{3}{4} \varepsilon V\right)^{2 / 3}\right] c_{1 y}(x, 0)
$$

As mentioned above, the parameter $a_{0}$ comes from boundary layer analysis. For convenience, the boundary condition will also be written as

$$
c_{1}(x, 0)=f\left(a_{0}, \varepsilon, V\right) c_{1 y}(x, 0)
$$


The linearization of the non-equilibrium slip condition proceeds along similar lines, utilizing the incompressibility condition to relate the derivatives of $u$ and $w$ at the EDL edge, or,

$$
\left.\frac{\partial u}{\partial x}\right|_{y=y_{0}}=-\frac{1}{8} V^{2}\left[\frac{c_{x x y} c_{y}-c_{x y}^{2}}{c_{y}^{2}}\right]_{y=y_{0}}=-\left.\frac{\partial w}{\partial y}\right|_{y=y_{0}}
$$

and to first order,

$$
\left[\frac{c_{x x y} c_{y}-c_{x y}^{2}}{c_{y}^{2}}\right]_{y=y_{0}} \approx \delta e^{\lambda_{\ell} t} c_{1 x x y}(x, 0)
$$

and thus

$$
w_{1 y}(x, 0)=\frac{1}{8} V^{2} c_{1 x x y}(x, 0)
$$

\section{Fourier series representation of perturbations}

Taking the no-flux boundary conditions and symmetry about the $x$-axis into consideration, the following form is chosen for the perturbations of the concentration,

$$
c_{1}(x, y)=\sum_{\ell=0}^{\infty} A_{\ell}(y) \cos \left(k_{\ell} x\right)
$$

where the wavenumber is given by

$$
k_{\ell}=\frac{2 \ell \pi}{h}
$$

Note the existence of a cutoff wavenumber for a non-trivial perturbation, $k_{\min }=2 \pi / h$ corresponding to a maximum wavelength along the $x$-direction equal to half the channel width. Substituting this form into equation 5.4 , the following form is obtained for $w_{1}(x, y)$,

$$
w_{1}(x, y)=\frac{1}{P e} \sum_{\ell=0}^{\infty}\left[A_{\ell}^{\prime \prime}(y)-A_{\ell}(y)\left(\lambda+k_{\ell}^{2}\right)\right] \cos \left(k_{\ell} x\right)
$$

It is worth taking a moment to consider the no-slip condition at $x= \pm h / 2$ in this problem. Evidently, the only solution corresponding to a single-mode concentration perturbation which satisfies this condition is the trivial solution for the hydrodynamic perturbation ${ }^{2}$.

\footnotetext{
${ }^{2}$ The coefficients $A_{\ell}$ which allow this boundary condition to be met for a single mode obey the 2 nd order ODE $A^{\prime \prime}-A\left(\lambda+k^{2}\right)=0$, but these solutions result in no hydrodynamic perturbation.
} 
Returning to the general case, the problem is transformed into a sixth-order linear homogeneous ODE for the $y$-dependent coefficients $A_{\ell}$,

$$
\begin{aligned}
A_{\ell}^{(6)}-A_{\ell}^{(4)}\left[3 k_{\ell}^{2}+\frac{\lambda_{\ell}}{S c}+\lambda_{\ell}\right]+A_{\ell}^{\prime \prime} & {\left[3 k_{\ell}^{4}+2 \lambda_{\ell} k_{\ell}^{2}+\frac{\lambda_{\ell}\left(2 k_{\ell}^{2}+\lambda_{\ell}\right)}{S c}\right] } \\
& -A_{\ell} k_{\ell}^{2}\left(k_{\ell}^{2}+\lambda_{\ell}\right)\left(k_{\ell}^{2}+\frac{\lambda_{\ell}}{S c}\right)=0
\end{aligned}
$$

Assuming a solution of form $A_{\ell}(y) \sim e^{m y}$, the characteristic equation is,

$$
\begin{aligned}
m^{6}-m^{4}\left[3 k_{\ell}^{2}+\frac{\lambda_{\ell}}{S c}+\lambda_{\ell}\right]+m^{2} & {\left[3 k_{\ell}^{4}+2 \lambda_{\ell} k_{\ell}^{2}+\frac{\lambda_{\ell}\left(2 k_{\ell}^{2}+\lambda_{\ell}\right)}{S c}\right] } \\
& -\left[k_{\ell}^{2}\left(k_{\ell}^{2}+\lambda_{\ell}\right)\left(k_{\ell}^{2}+\frac{\lambda_{\ell}}{S c}\right)\right]=0
\end{aligned}
$$

The eigenvalues of this equation are:

$$
\begin{aligned}
& m_{\ell 1}=-k_{\ell} \\
& m_{\ell 2}=k_{\ell} \\
& m_{\ell 3}=-\sqrt{k_{\ell}^{2}+\lambda_{\ell}} \\
& m_{\ell 4}=\sqrt{k_{\ell}^{2}+\lambda_{\ell}} \\
& m_{\ell 5}=-\frac{\sqrt{S c k_{\ell}^{2}+\lambda_{\ell}}}{\sqrt{S c}} \\
& m_{\ell 6}=\frac{\sqrt{S c k_{\ell}^{2}+\lambda_{\ell}}}{\sqrt{S c}}
\end{aligned}
$$

Thus the solution for the coefficients is

$$
A_{\ell}(y)=\sum_{n=1}^{6} a_{n \ell} e^{m_{\ell n} y}
$$

and the general concentration perturbation field,

$$
c_{1}(x, y)=\sum_{\ell=0}^{\infty} c_{1 \ell}(x, y)=\sum_{\ell=0}^{\infty} \sum_{n=1}^{6} a_{n \ell} e^{m_{\ell n} y} \cos k_{\ell} x
$$

and for the general fluid velocity perturbations,

$$
w_{1}(x, y)=\frac{1}{P e} \sum_{\ell=0}^{\infty} \sum_{n=1}^{6}\left\{a_{n \ell} e^{m_{n \ell} y}\left[m_{n \ell}^{2}-\left(\lambda+k_{\ell}^{2}\right)\right]\right\} \cos k_{\ell} x
$$




\section{Re-iteration of the boundary conditions and the algebraic solution for the $a_{n \ell}$}

For a specific perturbation of the concentration field given by a function $c_{1}(x, y)$ the coefficients $A_{\ell}(y)$ are just the Fourier series coefficients for this disturbance which also satisfy the 4th order PDE for $w_{1}$, so that

$$
A_{\ell}(y)=\frac{2}{h} \int_{0}^{h / 2} c_{1}(x, y) \cos k_{\ell} x d x=\sum_{n=1}^{6} a_{n \ell} e^{m_{n \ell} y}
$$

To perform linear stability analysis, it will be necessary to eliminate the coefficients $a_{n \ell}$ by solving for them using the boundary conditions. There are two boundary conditions in the $y$-direction in terms of $w_{1}$ and two in terms of $c_{1}$. In addition to the linearized nonequilibrium slip condition, the no-slip boundary condition at $y=1$, i.e. $u_{1}(x, 1)=0$ may be employed once it is cast in terms of the $A_{\ell}(y)$ to obtain another boundary condition. To do this, consider that the incompressibility condition may be integrated, using the no-flux condition at $x= \pm h / 2, u_{1}( \pm h / 2, y)=0$, so that the following integral can be written for the $x$-component of the fluid velocity,

$$
u_{1}(x, y)=\int_{x}^{h / 2} w_{1 y} d x
$$

This gives

$$
u_{1}(x, y)=-\frac{1}{P e} \sum_{\ell=0}^{\infty} \sum_{n=1}^{6}\left\{\frac{a_{n \ell} e^{m_{n \ell} y}}{k_{\ell}}\left[m_{n \ell}^{2}-\left(\lambda+k_{\ell}^{2}\right)\right]\right\} \sin k_{\ell} x
$$

The six boundary conditions for the problem in the $y$-direction for the $\ell$ th mode are given

below followed by the resulting expression given by the eigenvalue expansions for the $A$ (where Fourier's trick has been used.)

at $y=1$,

$$
\begin{array}{r}
c_{1 \ell}(x, 1)=0 \\
w_{1 \ell}(x, 1)=0 \\
u_{1 \ell}(x, 1)=0
\end{array}
$$


yielding the equations,

$$
\begin{aligned}
\sum_{n=1}^{6} a_{n \ell} b_{1 n \ell} & =\sum_{n=1}^{6} a_{n \ell} e^{m_{\ell n}}=0 \\
\sum_{n=1}^{6} a_{n \ell} b_{2 n \ell} & =\sum_{n=1}^{6} a_{n \ell} e^{m_{\ell n}}\left[m_{n \ell}^{2}-\left(\lambda+k_{\ell}^{2}\right)\right]=0 \\
\sum_{n=1}^{6} a_{n \ell} b_{3 n \ell} & =\sum_{n=1}^{6} \frac{a_{n \ell} m_{n \ell} e^{m_{\ell n}}}{k_{\ell}}\left[m_{n \ell}^{2}-\left(\lambda+k_{\ell}^{2}\right)\right]=0
\end{aligned}
$$

and at $y=0$,

$$
\begin{aligned}
c_{1 \ell}(x, 0) & =f\left(a_{0}, \varepsilon, V\right) c_{1 y}(x, 0) \\
w_{1 \ell}(x, 0) & =0 \\
w_{1 \ell y}(x, 0) & =\frac{1}{8} V^{2} c_{1 x x y}(x, 0)
\end{aligned}
$$

which gives,

$$
\begin{aligned}
\sum_{n=1}^{6} a_{n \ell} b_{4 n \ell} & ==\sum_{n=1}^{6} a_{n \ell}\left[1-m_{n \ell} f(a, \varepsilon, V)\right]=0 \\
\sum_{n=1}^{6} a_{n \ell} b_{5 n \ell} & =\sum_{n=1}^{6} a_{n \ell}\left[m_{n \ell}^{2}-\left(\lambda+k_{\ell}^{2}\right)\right]=0 \\
\sum_{n=1}^{6} a_{n \ell} b_{6 n \ell} & =\sum_{n=1}^{6} a_{n \ell} m_{n \ell}\left\{\frac{\left[m_{n \ell}^{2}-\left(\lambda+k_{\ell}^{2}\right)\right]}{P e}+\frac{1}{8} V^{2} k_{\ell}^{2}\right\}=0
\end{aligned}
$$

\section{Linear stability analysis}

To complete linear stability analysis, it seems necessary to solve for the $a_{n \ell}$ in the above equations, then substitute these expressions into the conditions and solve for $\lambda_{\ell}\left(k_{\ell}\right)$. However, the matrix $b_{m n, \ell}$ is square non-singular, so the equation (for given $\ell$ )

$$
b_{m n} a_{n}=\overrightarrow{0}
$$

has an empty nullspace.

To confound matters, if exchange of stabilities is assumed and the marginal case sought, i.e. by taking $\lambda_{\ell}=0$, the solution for $A_{\ell}(y)$ just becomes (dropping subscripts)

$$
A(y)=a_{1} \exp -k y+a_{2} \exp k y
$$


where the boundary condition on the concentration at $y=1$ implies $a_{1}=a_{2} \exp 2 k$. Note that, under the assumption of marginal stability, satisfying the final boundary condition requires $\exp 2 k=1$. If this holds, the boundary condition on the concentration at $y=0$ implies a contradiction.

Barring the (very reasonable) possibility of some as-of-yet unfound error in the calculations, the only conclusion that can be drawn from this attempt at stability analysis is that there exists a minimum wavenumber ${ }^{3}$ for perturbations corresponding to a maximum wavelength equal to the channel width.

\subsection{Discussion, conclusions, and future work}

While the preceding analysis is admittedly incomplete, it represents the first necessary steps towards obtaining a rigorous estimate of the threshold for the electro-osmotic instabilities believed to be responsible for overlimiting conductance in non-ideal permselective systems. In the process, several intermediate results of interest have been obtained, including evidence that the non-ideal membrane thresholds are shifted with respect to the ideal case, depending on the strength of the selectivity as measured by the factor $G$. Also, it was shown that the common assumption of local electro-neutrality inside a 1D permselective membrane is not compatible with the full formulation of the problem. Lastly, relating directly to the issue of instabilities, it was determined that there exists a minimum cut-off wavenumber stipulating a maximum wavelength for spatial perturbations. However, there are a variety of instabilities in electrokinetic systems related to limiting concentration gradients which may contribute to the process of diffusive length selection and overlimiting conductance, even if not directly responsible.

A common assumption is that the vortex-instability causes mixing with the bulk-like solution, thus enhancing the conductivity of the depleted regions and providing the source of the overlimiting conductance. Experimental evidence from the references $[53,5,66,67]$ as well as the NCM/NMI observations of chapter 3 supports this hypothesis. However, the fact that no single length selection criteria has been found that adequately describes the

\footnotetext{
${ }^{3}$ the uniform perturbation of the mode $\ell=0$ is excluded by the boundary conditions
} 
diffusion length suggests there are some open questions. Another issue is that the vortex arrays observed by Yossifon et. al. are relatively stable, whereas the experimental evidence for vortices in the microchannel - at least at high off-state voltages - suggest transient vortex formation and anihilation processes. This leads one to wonder whether or not some mechanism similar to a Rayleigh-Taylor instability is responsible for the mixing. Such an instability has been predicted in electrokinetic systems previously [6] under different assumptions than those employed here and in the other references. 


\section{Appendix A}

\section{Mathematica codes}

\section{A.1 Numerical evaluation for the 1D and quasi-1D LEN non-ideal solution}

The following is a version of the Mathematica code used to calculate the ion concentration at the right-hand membrane-electrolyte interface (output: peakconc.dat,) perm-selectivity as a function of voltage (output: permselectivev.dat,) and $I$ - $V$ characteristic (output: iv.dat,) given the input parameters for the system, shown along with the equivalent parameters from the model of Chapter 2 in Table A.1 below. A modified version of this can be used to determine potential profiles and concentration profiles. The main loop (see comments denoted by ' $(* *)$ ' in the code for explanation) for the modified version is shown in the next section. This version of the code can accommodate both length asymmetry, via the variable 'gamma,' as well as cross-sectional asymmetry via the variable 'bg.' This term modifies the reservoir salt flux density. Setting $b g=1$ yields length asymmetry only and setting gamma $=1$ yields flux-focusing only. Most of the other parameters appearing are step-sizes and internallygenerated values.

The code steps through values of the salt-flux density, solving the concentration profile inside the membrane numerically, which is then used to calculate the selectivity and cor- 
Table A.1: Model parameters from Chapter 2 and Mathematica variable names.

\begin{tabular}{ccc}
\hline Parameter & Variable name & Physical meaning \\
\hline$\gamma$ & gamma & Ratio of diffusion lengths \\
$\Gamma$ & bg & Ratio of cross-sections \\
$\mathcal{D}$ & $\mathrm{dm}$ & Effective membrane diffusivity \\
$N$ & num & Membrane fixed charge density \\
$\sigma$ & $\operatorname{sig}$ & Membrane conductivity \\
$\ell$ & 1 & Reservoir dimensionless DL length \\
\hline
\end{tabular}

responding voltage at that value of the salt-flux density after numerically integrating the inverse concentration profile to determine the membrane resistivity/conductivity term, $\mathfrak{F}(1)$.

Clear $[g, f$, phi, phileft, phiright, phimemb, jr, jt $]$

Clear[bg, gamma, $l$, eta, eqnb, $c, f$, jmin, jmax, jstep, jarray, etamax, etastart, cstart, cmin, cmax, dstep, jp, ivarray, psarray, clendarray, et]

$\left({ }^{*}\right.$ jstart $=$ jmin - jstep $\left./ 10000 *\right)$

gamma $=1.0$

$\mathrm{bg}=1$

$\mathrm{dm}=0.2$

num $=5.0$

$\mathrm{sig}=\mathrm{num} * \mathrm{dm}$

$l=4.0$

etamax $=1000000$

etastart $=5.0$

cstart $=$ num $/ 2$ 
cmin $=0.0$
cmax $=100 *$ num
dstep $=10000$

(* Definitions of V and I *)

$v\left[\mathrm{jp}_{-}\right.$, et_, $\left.\mathrm{f}_{-}\right]:=\log \left[\left(\operatorname{Sqrt}\left[(\mathrm{jp} *(l-1) / \mathrm{bg}+1)^{\wedge} 2+\operatorname{num}^{\wedge} 2 / 4\right]+\operatorname{num} / 2\right) /\left(\operatorname{Sqrt}\left[(1-\mathrm{jp} * \operatorname{gamma} *(l-1))^{\wedge} 2\right.\right.\right.$ + num $\left.^{\wedge} 2 / 4\right]+$ num $\left.\left./ 2\right)\right]-($ et +1$) * \log [(j p *(l-1) / \mathrm{bg}+1) /(1-\mathrm{jp} * \operatorname{gamma} *(l-1))]-\mathrm{jp} *$ et $* f / \mathrm{dm}$

$i\left[\mathrm{jp}_{-}\right.$, et_] $:=-2 *$ et $* \mathrm{jp}$

$\operatorname{jmin}=1 /(\operatorname{gamma} *(l-1))$

$\operatorname{jmax}=-\mathrm{bg} /(l-1)$

jstep $=($ jmin - jmax $) / 100$

jarray $=$ Table $[j,\{j, 25 *$ jstep,$-25 *$ jstep,- jstep $\}]$

prelista $=$ Table $[j,\{j, 49 *$ jstep, $30 *$ jstep,- jstep $/ 2\}]$

prelist $=$ Prepend $[$ prelista, jmin - jstep $/ 1000000]$

postlista $=$ Table $[j,\{j,-30 *$ jstep,$-49 *$ jstep,- jstep $/ 2\}]$

postlist $=$ Append[postlista, jmax + jstep $/ 1000000]$

jarray1 $=$ Join[prelist, jarray, postlist $]$

(* eqnb is obtained from Eqn. 3.4.5 at $x=1$ and solved numerically to obtain eta, the ratio of flux densities (see Chapter 3 for details.) *)

eqnb $=\operatorname{dm} *\left(\operatorname{Sqrt}\left[(\mathrm{jp} *(l-1) / \mathrm{bg}+1)^{\wedge} 2+\operatorname{num}^{\wedge} 2 / 4\right]-\operatorname{Sqrt}\left[(1-\mathrm{jp} * \operatorname{gamma} *(l-1))^{\wedge} 2+\operatorname{num}^{\wedge} 2 / 4\right]\right)$ $+\operatorname{sig} * \operatorname{eta} * \log \left[\left(\operatorname{Sqrt}\left[(j p *(l-1) / \mathrm{bg}+1)^{\wedge} 2+\operatorname{num}^{\wedge} 2 / 4\right]\right.\right.$

$-\operatorname{num} * \operatorname{eta} / 2) /\left(\operatorname{Sqrt}\left[(1-\text { jp } * \operatorname{gamma} *(l-1))^{\wedge} 2+\operatorname{num}^{\wedge} 2 / 4\right]-\operatorname{num} *\right.$ eta $\left.\left./ 2\right)\right] / 2==-$ jp $* 2$

eta $=$ Function $[\{j p\}$, eta/.FindRoot $[$ eqnb, $\{$ eta, etastart, 0, etamax $\}]]$ 
Plot[eta[jp], \{jp, (jmin - jmin/1000), (jmax + jmax/1000)\}]

runarray $=\{\operatorname{sig}, 1 /$ gamma $*(l-1),-b g /(l-1)\}$

Print["case: gamma = ",gamma, " dm = ", dm, " num = ", num, " $1=", l]$

CreateDirectory[

"C: $\backslash \backslash$ Users $\backslash \backslash$ online $\backslash \backslash$ Documents $\backslash \backslash$ Dissertation project folder $\backslash \backslash T H E$ FINAL CUT $\backslash \backslash$

Mathematica stuff for final version $\backslash \backslash$ flux focus only test $\backslash \backslash$ concentration profiles $\backslash \backslash$ gamma" <> ToString[gamma] <> " $\backslash \backslash$ membranediffusivity" <> ToString[dm] <> "\\membranecharge" <> ToString[num] <> " $\backslash$ diffusion length" <> ToString[l] <> " $\backslash \backslash$ Gamma" <> ToString[bg] <> "\\"]

\section{Export[}

\section{ToFileName[}

"C: $\backslash \backslash$ Users $\backslash \backslash$ online $\backslash \backslash$ Documents $\backslash \backslash$ Dissertation project folder $\backslash \backslash$ THE FINAL CUT $\backslash \backslash$

Mathematica stuff for final version $\backslash \backslash$ flux focus only test $\backslash \backslash$ concentration profiles $\backslash \backslash$ gamma" <> ToString[gamma] <> " \\membranediffusivity" <> ToString[dm] <>

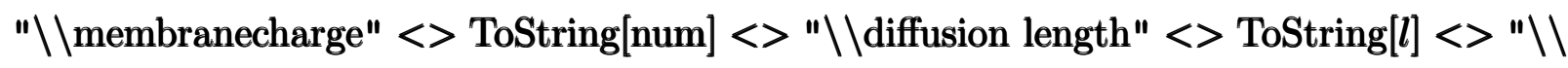
Gamma" <> ToString[bg] <> "\|sigmajminjmax.dat"], runarray, "Table"]

ivarray $=\{\}$

psarray $=\{\}$

clendarray $=\{\}$

(*MAIN LOOP: Loop over values of $\mathrm{J}_{-}+$to create an element $\left\{V\left[\mathrm{~J}_{-}+\right], I\left[\mathrm{~J}_{-}+\right]\right\}$for given eta, append to array.*)

$\operatorname{Do}[($ et $=$ eta[jp]; Print ["J+ = ",jp, " Jmin = ", 1/(gamma $*(l-1))$, " Jmax = ", -bg/(l-1)]; 
$c=$ Function $[\{z\}$,

$c /$. FindRoot $\left[\mathrm{dm} *\left(c-\operatorname{Sqrt}\left[(1-\mathrm{jp} * \operatorname{gamma} *(l-1))^{\wedge} 2+\operatorname{num}^{\wedge} 2 / 4\right]\right)+\right.$

$\operatorname{sig} *$ et $* \log \left[(c-\right.$ num $*$ et $/ 2) /\left(\operatorname{Sqrt}\left[(1-\text { jp } * \operatorname{gamma} *(l-1))^{\wedge} 2+\right.\right.$ num $\left.^{\wedge} 2 / 4\right]-$ num $*$ et $\left.\left./ 2\right)\right] / 2$

$==-\mathrm{jp} *(z+1),\{c$, cstart $, \mathrm{cmin}, \mathrm{cmax}\}]$;

values $=$ Table $[\{z, 1 / c[z]\},\{z,-1,1,1 /$ dstep $\}] ; \operatorname{cinv}=$ Interpolation[values $] ;$

$f=\operatorname{Integrate}[\operatorname{cinv}[z],\{z,-1,1\}]$;

ivarray $=$ Append[ivarray, $\{$ Evaluate $[v[\mathrm{jp}$, et,$f]]$, Evaluate $[i[\mathrm{jp}$, et $]]\}]$;

psarray $=$ Append $[$ psarray, $\{$ Evaluate $[v[j p$, et,$f]],-((1+$ et $) /(1-$ et $))\}] ;$ clendarray

$=$ Append[clendarray, $\left.\operatorname{Sqrt}\left[(1-\mathrm{jp} * \operatorname{gamma} *(l-1))^{\wedge} 2\right]\right]$;

Clear[et, $c, f$, values, cinv]), \{jp, jarray1\}]

ListPlot[ivarray, PlotRange->All]

ListPlot[psarray, PlotRange $\rightarrow$ All]

ListPlot[clendarray, PlotRange $\rightarrow$ All]

Export[

ToFileName[

"C: \\Users $\backslash \backslash$ online $\backslash \backslash$ Documents $\backslash \backslash$ Dissertation project folder $\backslash \backslash T H E$ FINAL CUT $\backslash \backslash$

Mathematica stuff for final version $\backslash \backslash$ flux focus only test $\backslash \backslash$ concentration profiles $\backslash \backslash$ gamma" <> ToString[gamma] <> " \\membranediffusivity" <> ToString[dm] <> "\\membranecharge" <> ToString[num] <> " \\diffusion length" <> ToString[l] <> "\\ Gamma" <> ToString[bg] <> "\\", "iv.dat"], ivarray, "Table"]

\section{Export[}

ToFileName[

"C: $\backslash \backslash$ Users $\backslash$ |online $\backslash \backslash$ Documents $\backslash \backslash$ Dissertation project folder $\backslash \backslash T H E$ FINAL CUT $\backslash \backslash$ Mathematica stuff for final version $\backslash \backslash$ flux focus only test $\backslash \backslash$ concentration profiles $\backslash \backslash$ gamma" <> ToString[gamma] <> " $\backslash \mid$ membranediffusivity" <> ToString[dm] <>

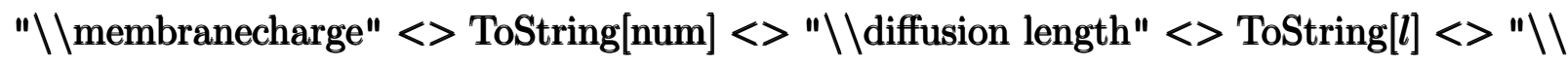
Gamma" <> ToString[bg] <> "\", "permselectivev.dat"], psarray, "Table"] 
Export[

\section{ToFileName[}

"C: $\backslash \backslash$ Users $\backslash \backslash$ online $\backslash \backslash$ Documents $\backslash \backslash$ Dissertation project folder $\backslash \backslash$ THE FINAL CUT $\backslash \backslash$

Mathematica stuff for final version $\backslash \backslash$ flux focus only test $\backslash \backslash$ concentration profiles $\backslash \backslash$ gamma" <> ToString[gamma] <> " $\backslash \backslash$ membranediffusivity" <> ToString[dm] <> "\|membranecharge" <> ToString[num] <> " $\backslash \backslash$ diffusion length" <> ToString[l] <> " $\backslash \backslash$ Gamma" <> ToString[bg] <> "\", "peakconc.dat"], clendarray, "Table"]

\section{A.2 Version of the code for plotting concentration and potential profiles}

This is just the main loop of the modified code. It solves Eqn. 3.4.5 twice, once at $x=1$ for fixed jp to obtain eta and once at a number of points $x_{i}$ between $x=-1$ and $x=1$ to obtain values for the $c_{m}\left(x_{i}\right)$. The latter provides a discrete approximation to the concentration profile. This is used directly to plot the concentration inside the membrane as well as interpolated and numerically integrated to give $\mathfrak{F}$.

$\operatorname{Do}[($ et $=$ eta[jp]; Print[" J+ = ",jp, " Jmin $=$ ", 1/gamma $*(l-1)$,

" Jmax = ", $-1 /(l-1)]$;

$c=$ Function $[\{z\}$,

$c /$.

FindRoot $\left[\mathrm{dm} *\left(c-\operatorname{Sqrt}\left[(1-\mathrm{jp} * \operatorname{gamma} *(l-1))^{\wedge} 2+\mathrm{num}^{\wedge} 2 / 4\right]\right)+\right.$ $\operatorname{sig} *$ et*

$\log [(c-\operatorname{num} *$ et $/ 2) /$

$\left(\operatorname{Sqrt}\left[(1-\mathrm{jp} * \operatorname{gamma} *(l-1))^{\wedge} 2+\operatorname{num}^{\wedge} 2 / 4\right]-\operatorname{num} *\right.$ et $\left.\left./ 2\right)\right] / 2==$

$-\mathrm{jp} *(z+1),\{c$, cstart, cmin, cmax $\}]]$;

ctable $=$ Table $[\{z, c[z]\},\{z,-1,1,1 /$ dstep $\}]$ 
Export[

ToFileName[

"C: $\backslash \backslash$ Users $\backslash \backslash$ online $\backslash \backslash$ Documents $\backslash \backslash$ Dissertation project

folder $\backslash \backslash$ Pieces $\backslash \backslash$ Code, data, and results $\backslash \backslash$ rectification

paper figures $\backslash \backslash$ concentration profiles $\backslash \backslash$ gamma" <>

ToString[gamma] <> " \\membranediffusivity" <> ToString[dm] <>

" \|membranecharge" <> ToString[num] <> " $\backslash \backslash$ diffusion length" <>

ToString $[l]<>$ " \\", "cmemb" <> ToString[jp] <> ".dat"], ctable,

"Table"]; values $=$ Table[ $\{z, 1 / c[z]\},\{z,-1,1,1 /$ dstep $\}]$;

$\operatorname{cinv}=$ Interpolation[values]; $f=\operatorname{Integrate}[\operatorname{cinv}[y],\{y,-1,1\}] ;$

$g=$ Function $[x$, Integrate $[\operatorname{cinv}[y],\{y,-1, x\}]]$

$\mathrm{vv}=$ Evaluate $[v[\mathrm{jp}$, et,$f]] ;$ Print $[\mathrm{vv}]$;

$\mathrm{jt}=$ Function $[r,($ et $* \log [\mathrm{jp} *(l-r)+1]+\mathrm{vv})]$;

Print[Evaluate[jt[0.2025]]];

$\mathrm{jr}=$

Function $[x$,

$(($ Evaluate $[i[\mathrm{jp}$, et $]] /(2 * \mathrm{dm})) * g[x]+$

$\log [\operatorname{Abs}[(1-$ jp $* \operatorname{gamma} *(l-1)) / c[x]]]+$

et $* \log [\operatorname{Abs}[(1-\mathrm{jp} * \operatorname{gamma} *(l-1))]])]$; Print[Evaluate[jr[0.2]]];

ptablea $=$ Table $[\{z$, et $* \log [1-$ jp $*(z+(\operatorname{gamma} *(l-1)+1))]\}$,

$\{z,-($ gamma $*(l-1)+1),-1,1 /$ dstep $\}]$;

ptableb $=$ Table $[\{x$, Evaluate $[\mathrm{jr}[x]]\},\{x,-1,1,1 /$ dstep $\}] ;$

ptablec $=$ Table $[\{r$, Evaluate $[\mathrm{jt}[r]]\},\{r, 1, l, 1 /$ dstep $\}]$;

\section{Export[}

ToFileName[

"C: $\backslash \backslash$ Users $\backslash \backslash$ online $\backslash \backslash$ Documents $\backslash \backslash$ Dissertation project folder $\backslash \backslash$ Pieces $\backslash \backslash$ Code, data, and results $\backslash \backslash$ rectification paper figures $\backslash \backslash$ concentration profiles $\backslash \backslash$ gamma" <> 
ToString[gamma] <> " $\backslash \backslash$ membranediffusivity" $<>$ ToString[dm] <> "\\membranecharge" <> ToString[num] <> " \\diffusion length" <> ToString $[l]<>$ " \\", "potentiala" <> ToString[jp] <> ".dat"], ptablea, "Table"];

Export[

ToFileName[

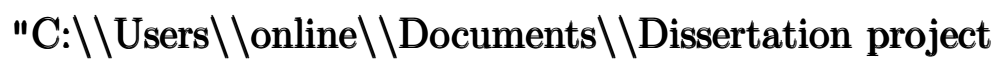
folder $\backslash \backslash$ Pieces $\backslash \backslash$ Code, data, and results $\backslash \backslash$ rectification paper figures $\backslash \backslash$ concentration profiles $\backslash$ |gamma" <> ToString[gamma] <> " \\membranediffusivity" <> ToString[dm] <> "\\membranecharge" <> ToString[num] <> "\\diffusion length" <> ToString $[l]<>$ " \\", "potentialb" <> ToString[jp] <> ".dat"], ptableb, "Table"];

Export[

ToFileName[

"C: $\backslash \backslash$ Users $\backslash \backslash$ online $\backslash \backslash$ Documents $\backslash \backslash$ Dissertation project folder $\backslash \backslash$ Pieces $\backslash \backslash$ Code, data, and results $\backslash \backslash$ rectification paper figures $\backslash \backslash$ concentration profiles $\backslash \backslash$ gamma" $<>$ ToString[gamma] <> " $\backslash \backslash$ membranediffusivity" <> ToString[dm] <> "\\membranecharge" <> ToString[num] <> " \\diffusion length" <> ToString $[l]<>$ " \\", "potentialc" <> ToString[jp] <> ".dat"],

ptablec, "Table"];

ivarray $=$ Append $[$ ivarray, $\{$ Evaluate $[v[\mathrm{jp}$, et, $f]]$, Evaluate $[i[\mathrm{jp}$, et $]]\}]$;

psarray $=$ Append $[$ psarray,

$\{$ Evaluate[ $v[\mathrm{jp}$, et, $f]],-((1+\mathrm{et}) /(1-\mathrm{et}))\}]$;

Clear[et, $c, f, g$, phi, values, cinv, ctable, ptablea, ptableb, 
ptablec, vv, jt, jr] $),\{$ jp, jmin, jmax, - jstep $\}]$

ListLinePlot[ivarray, PlotRange->All]

ListLinePlot[psarray, PlotRange $\rightarrow$ All]

Export[

ToFileName[

"C: \\Users $\backslash$ |online $\backslash \backslash$ Documents $\backslash \backslash$ Dissertation project

folder $\backslash \backslash$ Pieces $\backslash \backslash$ Code, data, and results $\backslash \backslash$ rectification

paper figures $\backslash \backslash$ concentration profiles $\backslash$ |gamma" $<>$

ToString[gamma] <> " \\membranediffusivity" <> ToString[dm] <>

"\\membranecharge" <> ToString[num] <> "\\diffusion length" <> ToString $[l]<>$ "\\", "iv.dat"], ivarray, "Table"]

Export[

ToFileName[

"C: $\backslash \backslash$ Users $\backslash \backslash$ online $\backslash \backslash$ Documents $\backslash \backslash$ Dissertation project

folder $\backslash \backslash$ Pieces $\backslash \backslash$ Code, data, and results $\backslash \backslash$ rectification

paper figures $\backslash \backslash$ concentration profiles $\backslash \backslash$ gamma" $<>$

ToString[gamma] <> " \\membranediffusivity" <> ToString[dm] <>

"\\membranecharge" <> ToString[num] <> " \\diffusion length" <>

ToString $[l]<>~ " \backslash \backslash$ ", "permselectivev.dat"], psarray, "Table"]

Clear[gamma, $l$, eta, eqnb, $c, f$, jmin, jmax, jstep, etamax, etastart, cstart, cmin, cmax, dstep, jp, ivarray, psarray, et] 


\section{References}

[1] M. Bazant, K. Thornton, and A. Ajdari, Phys. Rev. E. 70 (2004), 021506.

[2] S.H. Behrens and D.G. Grier, J. Phys. Chem. 115 (2001), 6716.

[3] W. Booth, K. Sapp, J. Schiffbauer, and B. Edwards, 2011.

[4] Brunet and A. Ajdari, Anal. Chem. 69 (1997), 4627.

[5] H. Chang and G. Yossifon, Biomicrofluidics 3 (2009), 012001.

[6] C. Chen, H. Lin, S. Lele, and J. Santiago, J. Fluid Mech. 524 (2005), 263.

[7] A. Collins, J. Schiffbauer, and B. Edwards.

[8] H. Daiguji, Y. Oka, and K. Shirono, Nano Lett. 5 (2005), 173.

[9] H. Daiguji, P. Yang, and A. Majumdar, Nano Lett. 4 (2004), 137-142.

[10] F. Van der Heyden, D. Stein, K. Besteman, S. G. Lemay, and C. Dekker, Phys. Rev. Lett. 96 (2006), 224502.

[11] R. Dhopeshwarkar, R. Crooks, D. Hlushkou, and U. Tallarek, Anal. Chem. 80 (2008), 1039-1048.

[12] S. Dukhin, Adv. Coll. Interf. Sci. 35 (1991), 173-196.

[13] C. Fuller, Rec. Chem. Progr. 17 (1956), 75.

[14] D. Gargas, D. Bussian, and S. Buratto, Nano. Lett. 5 (2005), 2184-2187.

[15] D. Hlushkou, R. Dhopeshwarkar, R. Crooks, and U. Tallarek, Lab Chip (2008), 1-10.

[16] K. Huang and R. Yang, Microfluid Nanofluid 5 (2008), 631-638.

[17] R. Hunter, Zeta potential in colloid science, principles and applications, Academic Press, 1981.

[18] _ Foundations of colloid science, vii., Oxford, 1989. 
[19] J. Jung, P. Joshi, L. Petrossian, T. Thornton, and J. Posner, Anal. Chem. 81 (2009), 3128.

[20] D. Kalkwarf, D. Frasco, and W. Brattain, Proc. Nat. Acad. Sci. 69 (1972), 3765-3768.

[21] E. Kalman, O. Sudre, I. Vlassiouk, and Z. Siwy, Anal. Bioanal. Chem. 394 (2009), $413-419$.

[22] R. Karnik, C. Duan, K. Castelino, H. Daiguji, and A. Majumdar, Nano Lett. 7 (2007), 547.

[23] R. Karnik, R. Fan, M. Yue, D. Li, P. Yang, and A. Majumdar, Nano Lett. 5 (2005), 943-948.

[24] K. Kelly, S. Miller, and A. Timperman, Anal. Chem. 81 (2009), 732.

[25] R. Kelly, Y. Li, and A. Woolley, Anal. Chem. 78 (2006), 2565-2570.

[26] S. Kim, Y. Wang, J. Lee, H. Jang, and J. Han, Phys. Rev. Lett. 99 (2007), 044501.

[27] T. Kuo, L. Sloan, J. Sweedler, and P. Bohn, Langmuir 17 (2001), 6298.

[28] V.G. Levich, Physicochemical hydrodynamics, Prentice Hall, 1962.

[29] H. Lin, B. Storey, M. Oddy, C. Chen, and J. Santiago, Phys. Fluids. 16 (2004), 1922.

[30] Q. Liu, Y. Wang, W. Guo, H. Ji, J. Xue, and Q. Ouyang, Phys. Rev. E. 579 (2007), 173.

[31] B. Lovrecek, A. Despic, and J. Bockris, J. Phys. Chem. 63 (1959), 750-751.

[32] J. Lyklema, Fundamentals of interface and colloid science, vi., Academic Press, Ltd., 1991.

[33] A. Mani, T. Zangle, and J. Santiago, Langmuir 25 (2009), 3898.

[34] _ Langmuir 25 (2009), 3909.

[35] J. Manzanares, W. Murphy, S. Mafe, and H. Reiss, J. Phys. Chem. 97 (1993), 8524.

[36] Mazur and Overbeek, Anal. Chem. 69 (195?), 4627.

[37] S. Miller, K. Kelly, and A. Timperman, Lab Chip 8 (2008), 1729.

[38] T. Narasimhan, Physics Today 62 (2009), 48.

[39] N. Nguyen and S. Wereley, Fundamentals and applications of microfluidics, Artech House, 2002.

[40] J. Piasecki, R. Allen, and J. Hansen, arXiv:cond-mat NA (2007), NA.

[41] A. Plecis, C. Nanteuil, A. Haghiri-Gosnet, and Y. Chen, Anal. Chem. 80 (2008), 9542. 
[42] R. Probstein, Physicochemical hydrodynamics, an introduction, Wiley, 2003.

[43] Q.Pu, J. Yun, H. Temkin, and S. Liu, Nano Lett. 4 (2004), 1099.

[44] D. Reguera and J.M. Rubi, Phys. Rev. E 64 (2001), 061106.

[45] H. Riess, J. Chem. Phys. 21 (1953), 1209.

[46] I. Rubinstein, Electrodiffusion of ions, SIAM, 1990.

[47] I. Rubinstein and L. Shtillman, J. Chem. Soc., Faraday Trans. II. 75 (1979), 231-246.

[48] I. Rubinstein and B. Zaltzman, personal correspondence and conversation.

[49] _ Math. Meth. Mod. App. Sci. 579 (1996), 623.

[50] - Math. Meth. Mod. App. Sci. 11 (2001), 263.

[51] _ Phys. Rev. E. 68 (2003), 032501.

[52] I. Rubinstein, B. Zaltzman, V. Gitis, and V. Nikonenko, Phys. Rev. E. 79 (2009), 021506 .

[53] I. Rubinstein, B. Zaltzman, J.Pretz, and C. Linder, Russ. J. Electrochem. 38 (2002), 853.

[54] J. Schiffbauer, E. Fernandez, K. Kelly W. Booth, B. Edwards, and A. Timperman.

[55] Z. Siwy, Adv. Funct. Mater. 16 (2006), 735.

[56] Z. Siwy, I. Kosinska, A. Fulinski, and C. Martin, Phys. Rev. Lett. 94 (2005), 048102.

[57] D. Stein, M. Kruithof, and C. Dekker, Phys. Rev. Lett. 93 (2004), 035901.

[58] G. Taylor, Proc. Roy. Soc. A (1953), 186-203.

[59] I. Vlassiouk, S. Smirnov, and Z. Siwy, Nano Lett. 8 (2008), 1978-1985.

[60] I. Vlassiouk, S. Smirnov, and Zuzanna Siwy, ACSNano 2 (2008), 1589.

[61] Y. Wang, A. Stevens, and J. Han, Anal. Chem. 77 (2005), 4293-4299.

[62] C. Wei and A. Bard, Anal. Chem. 69 (1997), 4627.

[63] D. Woermann, Cellular and Molecular Biology Lett. 6 (2001), 53.

[64] G. Yossifon and H. Chang, Phys. Rev. Lett. 101 (2008), 254501.

[65] G. Yossifon, Y. Chang, and H. Chang, Phys. Rev. Lett. 101 (2008), 254501.

[66] G. Yossifon, P. Mushenheim, Y.Chang, and H. Chang, Phys. Rev. E. 79 (2009), 046305 . 
[67] _ Phys. Rev. E. 81 (2010), 046301.

[68] B. Zaltzman and I. Rubinstein, J. Fluid Mech. 579 (2007), 173.

[69] R. Zwanzig, J. Phys. Chem. 96 (1992), 3926.

John H.

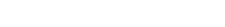
Hagen 